\title{
Implementierung und bodenökologische Charakterisierung von Kurzumtriebsplantagen und Agroforstsystemen am Beispiel der Bioenergie-Regionen "Göttinger Land" \& "Thüringer Ackerebene"
}

\author{
Dissertation \\ zur Erlangung des akademischen Grades Doctor of Philosphy (PhD) \\ der Fakultät für Forstwissenschaften und Waldökologie \\ der Georg-August-Universität Göttingen
}

vorgelegt von

Linda Hartmann

geboren in Sangerhausen

Göttingen, 2014 
1. Gutachter: Herr Prof. Dr. Norbert Lamersdorf

2. Gutachter: Herr Prof. Dr. Achim Dohrenbusch

Tag der mündlichen Prüfung: 27.10.2014 


\section{Vorwort}

Die vorliegende Arbeit entstand zwischen November 2010 und Juli 2014 im Rahmen des vom Bundesministerium für Bildung und Forschung geförderten Verbundprojektes "Bioenergie-Regionen stärken" an der Abteilung Ökopedologie der gemäßigten Zonen der Georg-August-Universität Göttingen.

\section{Danken möchte ich ...}

... herzlich meinem Betreuer und Themensteller Herrn Prof. Lamersdorf für sein Engagement und seine unermüdliche Hilfsbereitschaft.

... gern Herrn Prof. Dohrenbusch für die Begutachtung der Dissertation.

... Herrn Prof. Kuzyakov für die Möglichkeit in seiner Abteilung diese Arbeit anfertigen zu können.

... den technischen Mitarbeiterinnen der Abteilungen Ökopedologie der gemäßigten Zonen, Ökopedologie der Tropen und Subtropen sowie der Agrarpedologie der Universität Göttingen.

... besonders Herrn Dirk Böttger für seine Hilfe bei der Feldarbeit.

... allen Projektpartnern und Kollegen für die gute Zusammenarbeit während der Projektdauer.

... insbesondere Herrn Dr. Falk Richter und Herrn Dr. Martin Jansen für die wissenschaftliche Betreuung.

... von Herzen meiner Familie und meinen Freunden. 


\section{Inhaltsverzeichnis}

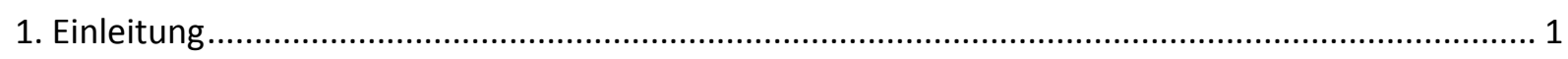

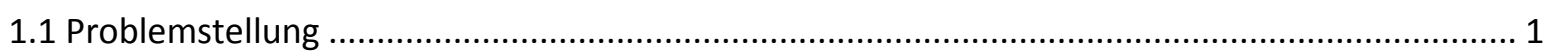

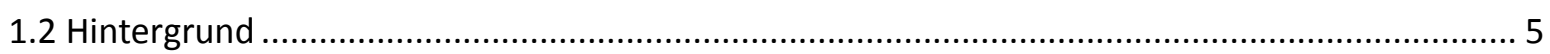

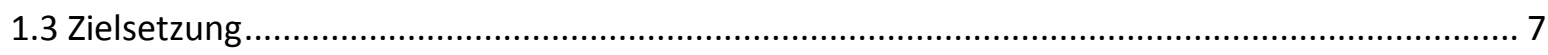

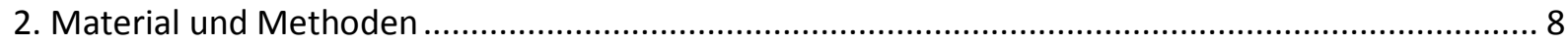

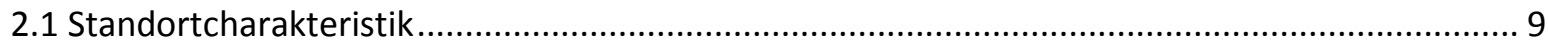

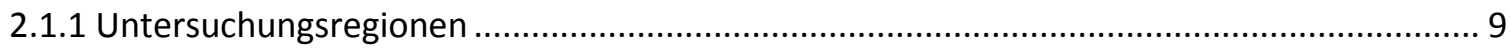

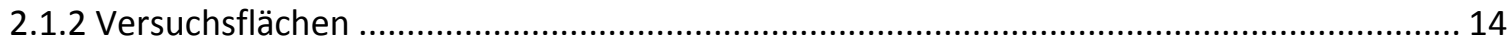

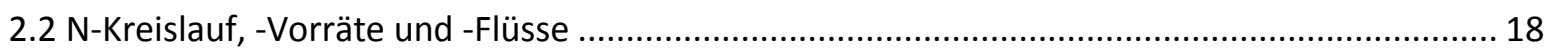

2.2.1 Extrahierbarer mineralischer und organischer Stickstoff................................................... 19

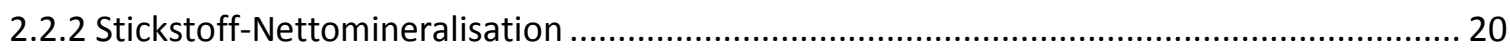

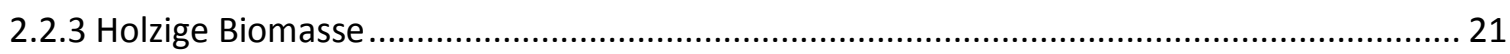

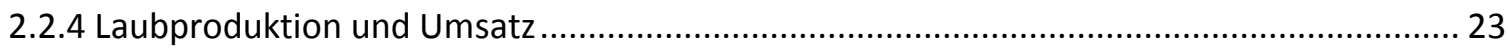

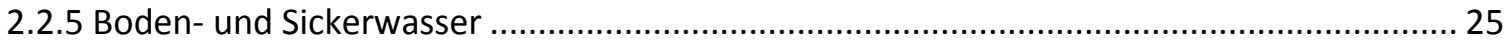

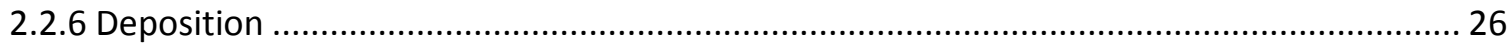

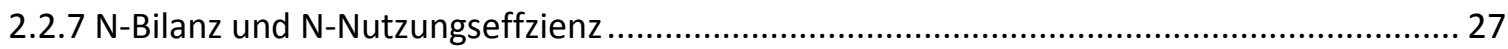

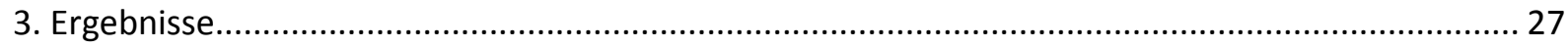

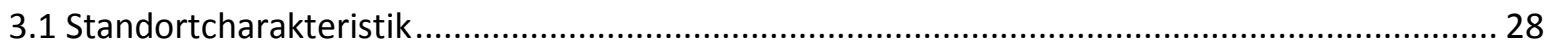

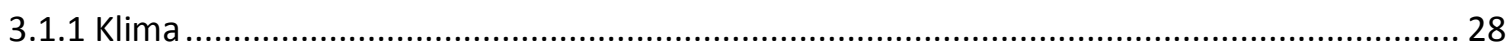

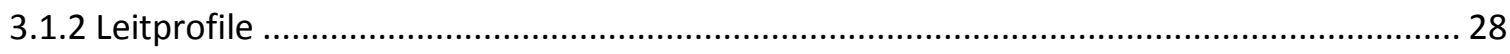

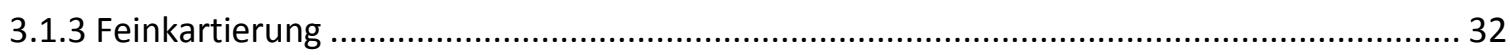

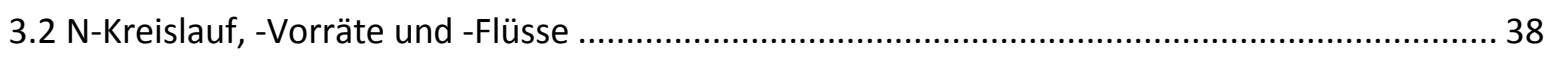

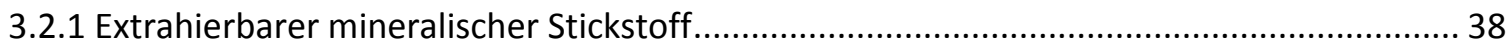

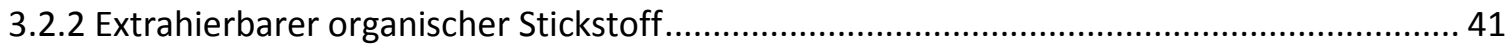

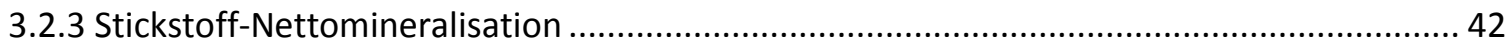

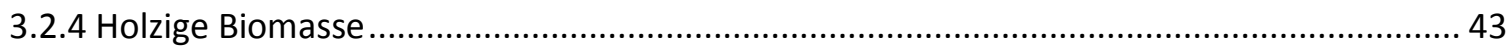

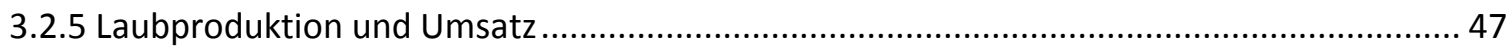

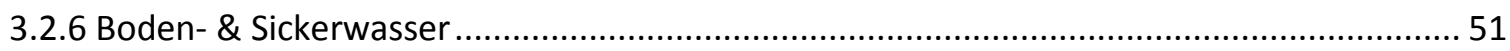

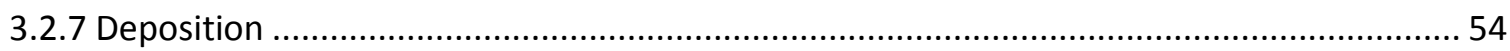

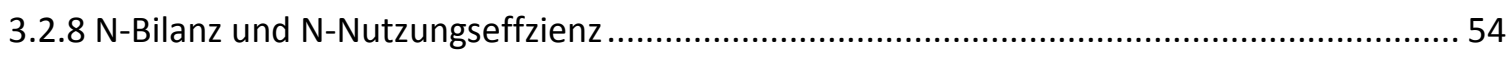

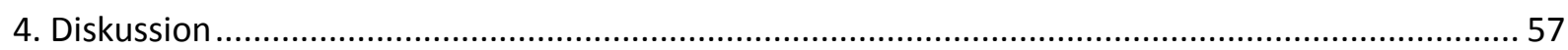

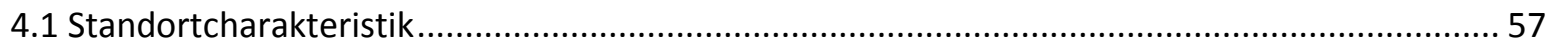

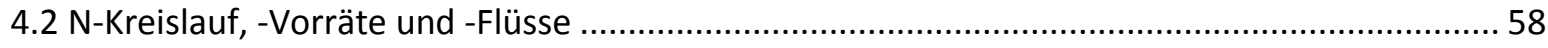




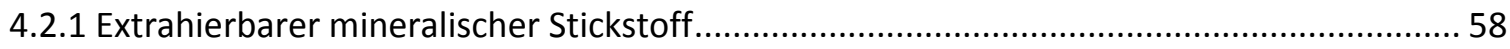

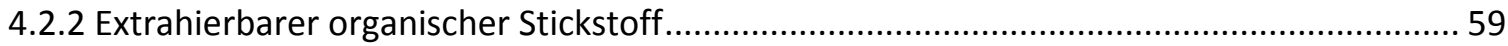

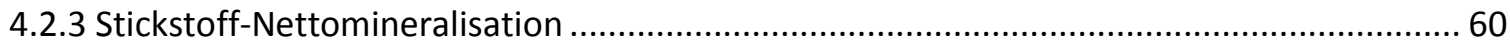

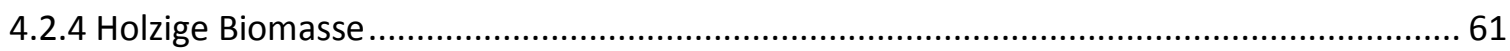

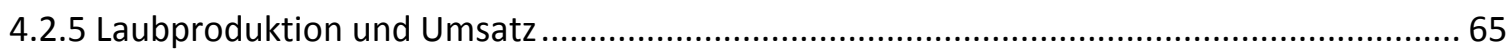

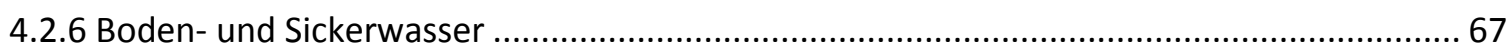

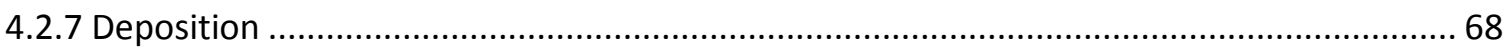

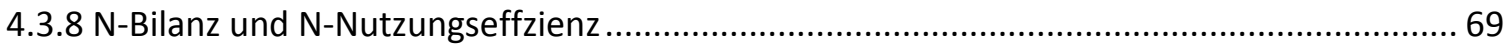

5. Schlussfolgerungen und Empfehlungen für die Praxis ..................................................................... 71

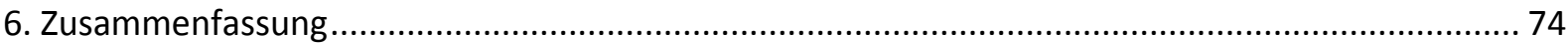

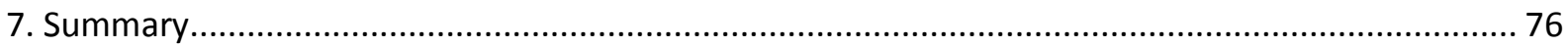

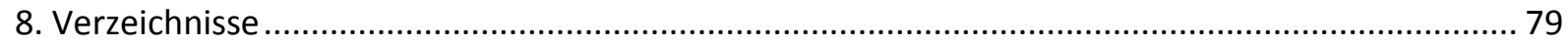

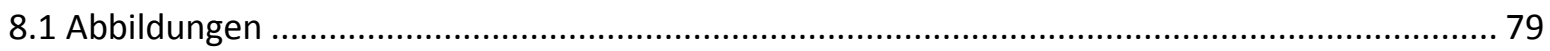

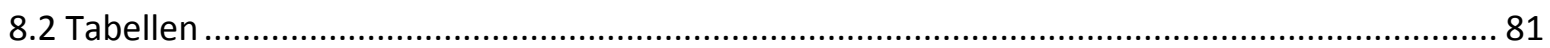

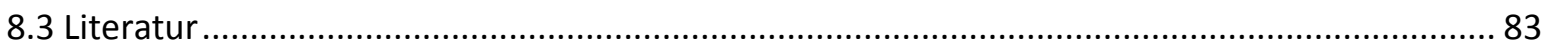

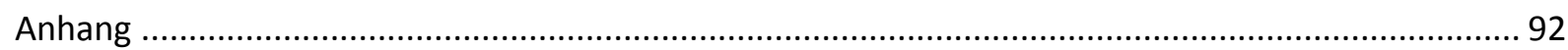




\section{Einleitung}

\subsection{Problemstellung}

Vor dem Hintergrund des Klimawandels und der Verknappung an fossilen Energieträgern soll der Anteil der erneuerbaren Energien am Endenergieverbrauch in Deutschland gesteigert werden und bis zum Jahr 2020 mindestens 20 \% betragen (Bemmann et al. 2007, Bemmann et al. 2010). Dabei kann die Biomassenutzung wesentlich zur Sicherung der Energieversorgung beitragen (Aretz u. Hirschl 2008, Bemmann et al. 2010). Staatliche Anreize wie das Erneuerbare-Energien-Gesetz (EEG) oder das Erneuerbare-Energien-Wärmegesetz (EEWärmeG) sorgen schon jetzt für einen verstärkten Anbau von Pflanzen zur Biomasseproduktion, um diese energetisch zu verwerten (Aretz u. Hirschl 2008).

Die Steigerung der Biomasseproduktion ist allerdings nicht umstritten. Da nur eine limitierte Gesamtfläche für die landwirtschaftliche Nutzung zur Verfügung steht, kann der zunehmende Anbau von Energiepflanzen zu einer Konkurrenzsituation zwischen der Biomasseproduktion und der Nahrungsmittelproduktion führen (Landgraf et al. 2007, Rösch u. Jorissen 2012). Zudem wird intensiv diskutiert, ob eine gesteigerte Biomasseproduktion auch negative Auswirkungen haben kann, beispielsweise auf die Artenvielfalt oder das Landschaftsbild (Nationale Akademie der Wissenschaften 2012, 2013). Unklar ist auch noch, in welcher Weise der Klimawandel die erwünschte Steigerung der Biomasseproduktion beeinflussen wird.

Es gibt verschiedene Möglichkeiten Biomasse auf regionaler Ebene, zur stofflichen und energetischen Verwertung, zu produzieren. Neuere Ansätze integrieren Gehölze auf landwirtschaftlichen Nutzflächen in Form von Kurzumtriebsplantagen (KUP) und Agroforstsysteme (AF). Dabei versteht man unter KUP den Anbau schnellwachsender Gehölze wie Pappel und Weide auf landwirtschaftlichen und vormals primär ackerbaulich genutzten Flächen, die im mehrjährigen Rhythmus und nach Wiederaustrieb durch Stockausschlag geerntet werden. Solange KUP in einem Turnus von 20 Jahren mindestens einmal geerntet werden, gilt diese Form der Landnutzung nach derzeitigem Recht weiterhin als landwirtschaftliche Flächennutzung (Dauerkultur) und nicht als Aufforstung (Bärwolff et al. 2012). Ziel des KUP-Anbaus ist die Erzeugung von holziger Biomasse zur thermischen oder stofflichen Verwertung. Eine spezielle Variante des KUP-Anbaus sind so genannte Agroforstsysteme, eine Mischung von KUP-Streifen mit Ackerkulturen oder Grünland auf der gleichen landwirtschaftlichen Fläche (Hartmann et al. 2014). Bisher existieren in Deutschland ca. 4.000 ha an KUP, wobei noch erhebliche Flächenpotenziale zum Ausbau dieser Anbausysteme bestehen (Rösch u. Jorissen 2012).

KUP und AF ermöglichen nicht nur eine ertragreiche Biomasseproduktion, sondern stellen darüber hinaus wichtige ökologische Serviceleistungen sicher. Sie dienen der:

- Förderung der Biodiversität, 
- Verbesserung des Klima-, Boden- und Wasserschutzes (Lamersdorf u. Busch 2010, Lamersdorf et al. 2010, Tsonkova et al. 2012).

Um diese Leistungen optimal zu gewährleisten, bedarf es einer zielgerichteten und standortgerechten Planung, Anlage und Bewirtschaftung von KUP und AF.

Zum erfolgreichen Anbau schnellwachsender Gehölze in KUP und AF müssen zunächst passende Pflanzmaterialen gewählt werden (Petzold et al. 2006, Röhle et al. 2008). Besonders geeignet sind Arten, die über ein schnelles Jugendwachstum, eine hohe Anwuchssicherheit, leichte Vermehrbarkeit und ein gutes Stockausschlagvermögen verfügen (Landgraf et al. 2010, Murach et al. 2009, Röhle et al. 2008). Vor allem die Arten der Gattungen Populus L. und Salix L. haben sich unter den mitteleuropäischen Standortbedingungen bewährt (Röhle et al. 2008). Diese stellen jedoch im Vergleich zu den meisten forstlichen Hochwaldbaumarten hohe Ansprüche an ihren Standort (Petzold 2010, Röhle et al. 2008).

Um möglichst hohe Erträge beim Anbau von Pappeln und Weiden zu erzielen, sollten die Anbaustandorte eine gute Nährstoff-, Wärme- und Wasserversorgung aufweisen (Petzold et al. 2006). Um einen ausreichenden Wärmehaushalt sicher zu stellen, sollte die Lufttemperatur im Jahresdurchschnitt mindestens $6,5^{\circ} \mathrm{C}$ betragen (Aust 2012, Petzold et al. 2010a). Neben einem ausreichenden Wärmeangebot ist auch die Versorgung der Pflanzen mit Wasser bedeutsam. Standorte mit einer Jahresniederschlagssumme von mindestens $500 \mathrm{~mm}$, davon mindestens $300 \mathrm{~mm}$ in der Vegetationsperiode (Mai-September) sollten für hohe Zuwachsraten $\left(>8 t_{\text {atro }} h^{-1} a^{-1}\right)$ der Pappeln und Weiden ausreichend sein (Petzold et al. 2006, Petzold et al. 2010a). Ferner sollte das Nährstoffangebot hoch sein, sodass nur eutrophe bis mesotrophe Standorte mit pH-Werten größer als 5 geeignet erscheinen (Liebhard 2007).

Die Stickstoffverfügbarkeit ist dabei meist der Faktor, der die Ertragsleistung von Weiden in KUP limitiert (Balasus et al. 2012, Jug et al. 1999b). Daher ist die Verfügbarkeit an Stickstoff (N), neben der Wasserversorgung, besonders bedeutsam für das Erreichen hoher Erträge. Demzufolge wurde Stickstoff im Hinblick auf seine Transformationen, Anreicherungen und Verarmungen in KUP und AF in dieser Arbeit analysiert (Abb. 1). 


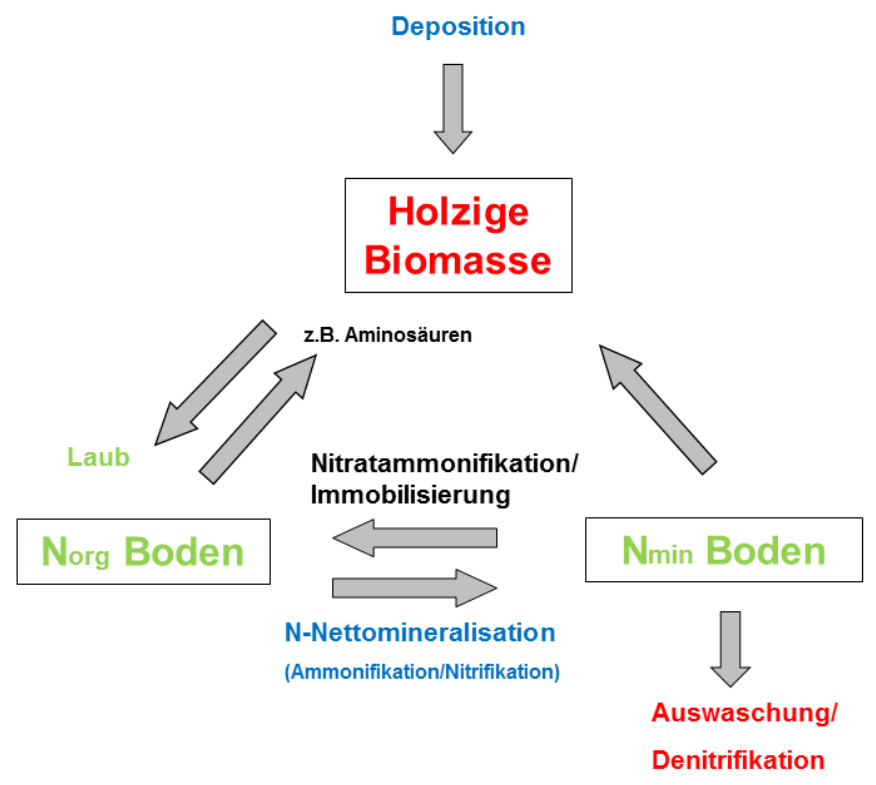

Abb. 1 Vereinfachter Stickstoffkreislauf (N-Kreislauf) mit Angaben zu Vorräten und Flüssen, die in dieser Arbeit bestimmt bzw. modelliert wurden, in grün: $\mathrm{N}-$ Vorrat $\left(\mathrm{N}_{\text {org }}=\right.$ organischer Stickstoff [eON], $\mathrm{N}_{\min }=$ mineralischer Stickstoff [eIN], blau: N-Eintrag (Deposition und N-Nettomineralisation) und rot: N-Austrag (Auswaschung mit dem Sickerwasser, Denitrifikation: Lachgasemission und Ernte der holzigen Biomasse)

Mehr als $90 \%$ der Bodenstickstoffvorräte liegen in organisch gebundener Form vor und stammen aus Vegetationsrückständen, Bodenorganismen sowie deren Stoffwechselprodukte (Blume et al. 2010). Durch die Prozesse der Ammonifikation und Nitrifikation wird aus dem organischen N-Vorrat mineralischer Stickstoff in Form von Ammonium und Nitrat freigesetzt (Lammel u. Flessa 1998). Günstige Bedingungen für die Ammonifikation sind mittlere Bodenwassergehalte (pF 2,7-4,2) und hohe Temperaturen (bis etwa $50^{\circ} \mathrm{C}$ ) (Gisi 1997). Der pH-Bereich sollte zwischen 5 und 8 liegen (Blume et al. 2010). Für eine optimale Nitrifikation sind eine ausreichende Sauerstoffversorgung, eine neutrale bis schwach saure Bodenreaktion, mittlere Bodenwassergehalte ( $\mathrm{pF}$ 2,7-4,2) und Temperaturen zwischen $25^{\circ} \mathrm{C}$ und $35^{\circ} \mathrm{C}$ Voraussetzung (Blume et al. 2010, Gisi 1997). Niedrige Temperaturen $\left(<6^{\circ} \mathrm{C}\right)$ oder eine unzureichende Belüftung (z.B. in Stau- oder Grundwasserhorizonten) begünstigen den Prozess der Ammonifikation gegenüber der Nitrifikation (Blume et al. 2010).

Einen Teil des organischen Stickstoffs benötigen die Mikroorganismen für sich selbst, sodass bei ungenügender N-Freisetzung, auch ein Teil des mineralischen Stickstoffs von den Mikroorganismen konsumiert werden kann (Immobilisierung) (Blume et al. 2010, Powlson 1993). Immobilisierung findet vor allem bei organischen Substanzen mit weitem C/N-Verhältnis statt (Powlson 1993), hingegen kommt es zur N-Freisetzung durch Ammonifikation bei Ausgangsstoffen mit engen $\mathrm{C} / \mathrm{N}$ Verhältnissen (C/N-Verhältnis 5 bis 20) (Blume et al. 2010). Eine optimale Nitrifikation, also die vollständige Umwandlung von Ammonium in Nitrat findet statt, wenn ein C/N-Verhältnis kleiner 25 vorliegt (Blume et al. 2010). 
Pflanzen können sowohl Ammonium und Nitrat, als auch niedermolekulare organische Stickstoffverbindungen aufnehmen (Blume et al. 2010, Korhonen et al. 2013, Rennenberg et al. 2009). Bei Sauerstoffmangel können sich größere Mengen an Ammonium im Boden anreichern, weil der Nitrifikationsprozess gehemmt ist (Gisi 1997). Ferner kann auch das Nitrat als Sauerstoffquelle von den Mikroorganismen genutzt werden (Denitrifikation) und als Stickstoffgas $\left(\mathrm{NO}, \mathrm{N}_{2} \mathrm{O}, \mathrm{N}_{2}\right.$ ) entweichen. Die Denitrifikation findet vorwiegend bei hohen Bodenwassergehalten (>70\%) und eingeschränkter Durchlüftung statt (Blume et al. 2010).

Ein weiterer Prozess der unter anaeroben Bedingungen stattfinden kann, ist die Reduktion von Nitrat zu Ammonium (Nitratammonifikation) (Blume et al. 2010). Die Nitratammonifikation ist an stark reduzierende Bedingungen gebunden, die in Ackerböden selten auftreten. Außerdem wird dem Boden Stickstoff durch die N-Aufnahme der Pflanzen entzogen. Die dem Boden entzogene N-Menge kann dabei über den Biomasseertrag berechnet werden (Kreutzer et al. 2009).

Zusätzlich zum bodenbürtigen Ammonium und Nitrat wird die verfügbare N-Menge im Boden durch trockene und nasse N-Deposition erhöht. Nitrat wird im Gegensatz zu Ammonium nur in geringem $\mathrm{Maß}$ an Bodenpartikel gebunden und kann daher mit dem Sickerwasser ausgewaschen werden. Eine weitere Quelle ist die biologische $\mathrm{N}_{2}$-Fixierung durch freilebende oder mit Pflanzen in Symbiose lebende Mikroorganismen (Gisi 1997). Mit dem Streufall und dem Absterben der Wurzeln und Organismen wird dem Boden wieder Stickstoff in organischer Form zugeführt (Hein 2003).

Ökosysteme funktionieren langfristig und nachhaltig nur nach dem Prinzip der geschlossenen Nährstoffkreisläufe, d.h. die Nährstoffausträge (z.B. Lachgas, Nitrat im Sickerwasser) sind sehr gering und stehen im Gleichgewicht mit der Nachlieferung (z.B. Eintrag mit dem Niederschlag) (Kölling et al. 2007). So weisen natürliche Waldökosysteme in der Regel einen geschlossenen N-Kreislauf mit einer annährend ausgeglichenen N-Bilanz auf (Kölling et al. 2007). Im Detail sind diese Systeme durch einen geringen Eintrag und Austrag und durch eine intensive Zirkulation (z.B. Laub) von Stickstoff zwischen den verschiedenen N-Vorräten (z.B. pflanzlicher und bodenbürtiger N-Vorrat) charakterisiert (Lammel u. Flessa 1998, Kölling et al. 2007, Korhonen et al. 2013, Rennenberg et al. 2009, Rennenberg et al. 2010). Dagegen sind Agrarökosysteme durch einen großen Eintrag (z.B. Düngung), Entzug (z.B. Ernte) und Austrag an Stickstoff (z.B. Nitrat im Sickerwasser) gekennzeichnet (Kölling et al. 2007, Rennenberg et al. 2009).

Für KUP auf landwirtschaftlichen Nutzflächen ergeben sich im Vergleich zu annuellen Anbauten andere N-Kreisläufe (Lamersdorf u. Busch 2010, Lamersdorf et al. 2010). Durch die Ausbildung eines tiefreichenden Wurzelsystems können größere Nährstoffreserven erschlossen werden. Über das Kronendach werden Nähr- aber auch Schadstoffe aus der Luft gefiltert, ein Großteil der Nährstoffe zirkuliert über die jährliche Blattstreu und verbleibt somit im System (Lamersdorf u. Busch 2010, Lamersdorf et al. 2010). Demzufolge liegt der Bedarf an Nährelementen deutlich unter dem von Ackerfrüchten (Lamersdorf et al. 2010). 
KUP weisen häufig einen geschlossenen N-Kreislauf auf, d.h. sie binden den aus der Atmosphäre eingetragenen Stickstoff, wodurch es aber in der Regel nicht zu einer N-Akkumulation oder zu unerwünschtem N-Austrag kommt (Strohm et al. 2012). Dennoch wird dem System regelmäßig Stickstoff durch die Holzernte entzogen. Werden diese N-Verluste nicht in gleicher Größenordnung wieder zugeführt, wird sich der N-Vorrat und somit die Fruchtbarkeit des Bodens verringern (Kölling et al. 2007). Damit die holzigen Anbausysteme (KUP und AF) auf landwirtschaftlichen Nutzflächen möglichst langfristig und nachhaltig bewirtschaftet werden können, sollte eine nahezu ausgeglichene N-Bilanz, vergleichbar zu natürlichen Waldökosystemen angestrebt werden.

\subsection{Hintergrund}

Im Rahmen der Untersuchungen zur Steigerung der regionalen Biomasseproduktion wird seit Ende 2010 das Verbundprojekt "Bioenergie-Regionen stärken" (BEST) durchgeführt. Insgesamt gliedert sich BEST in die folgenden sieben übergeordneten Forschungsbereiche ("so genannte Cluster"):

- Ökologische Landschaftsfunktionen

- Systemlösungen für die Mobilisierung von Holzreserven

- Innovative Anbausysteme und -verfahren

- Systemlösungen für eine stofflich-energetische Kaskadennutzung

- Ökologische Folgenabschätzung unterschiedlicher Bewirtschaftungsformen

- Sozioökonomische Bewertung von Nutzungskonzepten

- Ergebnisintegration, Umsetzung und Partizipation.

Jedem Cluster sind mehrere Teilprojekte zugeordnet, insgesamt beinhaltet BEST 31 Teilprojekte. Das Ziel von BEST ist es, regional angepasste Konzepte und innovative Systemlösungen zur Produktion von Biomasse zu entwickeln und im Hinblick auf ökologische und ökonomische Auswirkungen anhand zweier Bioenergie-Regionen ("Göttinger Land", Südniedersachsen und "Thüringer Ackerebene", Mittelthüringen) zu bewerten. Demzufolge wurden die Forschungsarbeiten von Anfang an in die Entwicklung zweier Bioenergie-Regionen eingebunden, die beispielhaft für den aktuellen Prozess der Formierung solcher Regionen überall in Deutschland stehen: der Landkreis Göttingen in Südniedersachsen (Ldk. Gö.) und der Verbund "BERTA" (BioEnergie Region Thüringer Ackerebene) in der "Thüringer Ackerebene" (Hartmann et al. 2014).

Die Bioenergie-Region "Göttinger Land" und die "Thüringer Ackerebene" sind zwei für Deutschland charakteristische Regionen, die sich hinsichtlich ihres naturräumlichen Charakters (Boden, Klima) und ihrer Landschaftsstruktur (Waldanteil, Siedlungsstruktur) unterscheiden (Hartmann et al. 2014). 
Im Landkreis Göttingen gab es bis zum Start der BEST-Initiative keinerlei KUP und AF, zudem gestaltete sich die Suche nach geeigneten Flächen für eine Neuanlage als schwierig. Da viele Teilprojekte des BEST-Verbundvorhabens (z.B. "Bioklimatologie", "Wasserhaushalt", "Klimaschutzleistung", "Landschaftsökologie" usw.) auf den Versuchsflächen ihren individuellen Fragestellungen nachgehen wollten, wurde ein Flächenkomitee gebildet, das sich mit der Ausarbeitung eines Anforderungskatalogs an die Versuchsflächen sowie der darauf aufbauenden Flächenauswahl beschäftigte.

Der Anforderungskatalog zur Auswahl geeigneter KUP- und AF-Flächen umfasst schließlich folgende zu erfüllende Kriterien:

- $\quad$ angemessene Flächengröße von mindestens 1 ha

- günstige Zufahrts- und Wendemöglichkeiten

- $\quad$ keine Grundwasser beeinflussten Standorte

- $\quad$ keine Sonderstandorte (z.B. Halden)

- fruchtbarer, homogener und gut durchwurzelbarer Boden

- geringe Hangneigung, um den Einsatz von Maschinen zu ermöglichen

- unmittelbare Nähe zu Flächen mit annuellen Energiepflanzen, Wald und Grünland

- $\quad$ Ackerzahl > 20 .

Letztendlich wurde aus den vier besichtigten Flächen im Ldk. Gö. die ca. 1,7 ha große Ackerfläche in Reiffenhausen für die Anlage von zwei KUP und eines Agroforstsystems (Kombination KUP-Streifen und Grünland) ausgewählt. Grundlage dieser Entscheidung war die unmittelbare Nähe der ausgewählten Fläche zum Biotopverbund "Grünes Band", also der ehemaligen innerdeutschen Grenze zu Thüringen. Diese stellte aus landschaftsökologischer Sicht eine optimale Fortsetzung des unmittelbar angrenzenden Grünlandsreifens des neu angelegten Agroforstsystems dar.

In der "Thüringer Ackerebene" konnte hingegen bei der Anlage der Versuchsfläche an bereits vorhandene Aktivitäten und Erfahrungen hinsichtlich des Anbaus von KUP angeknüpft werden (Hartmann et al. 2014). Hier wurden durch die Neuanlage von KUP mit Pappel oder Weide sowie einem AF die bestehenden Flächen so erweitert, dass eine Vergleichbarkeit zwischen den beiden Untersuchungsregionen gewährleistet war. Die Agroforstsysteme wurden möglichst in Nord-SüdRichtung mit dem Ziel einer maximalen Lichtausnutzung ausgerichtet.

Vor der Anlage der Versuchsflächen wurden Detailpläne zur genauen Lage von Zufahrtswegen, Flächengrößen, Zäunen und Vorgewenden ausgearbeitet.

In Anlehnung an die positiven Erfahrungen (Anwuchserfolg und Ertragsleistung) im Rahmen des Verbundprojektes "ProLoc" (Erfassung von Klon-Standort-Wechselwirkungen bei Pappel und Weide auf landwirtschaftlichen Standorten in kurzen Umtriebszeiten) wurde der Pappelklon "Max 1" und der Weidenklon "Tordis" auf den neu angelegten Flächen angepflanzt. Der Pflanzverband und die 
Pflanzzahl richtete sich nach der geplanten Umtriebszeit von maximal 3 Jahren und dem Projektziel möglichst viel Biomasse in kurzer Zeit zu produzieren, die maschinell geerntet werden kann.

Im Detail setzten sich die Versuchsflächen Reiffenhausen und BERTA schließlich aus jeweils zwei KUP (KUP-Pappel, KUP-Weide) und einem AF, das aus Grünlandstreifen (AF-Grünland) und Weidenstreifen (AF-Weide) bestand, zusammen (Abb. 2). Als Kontrollflächen zu den holzigen Anbausystemen KUP und AF wurden die unmittelbar angrenzenden Ackerflächen, mit den Kulturen Raps im Ldk. Gö. und Mais in der "Thüringer Ackerebene", genutzt.

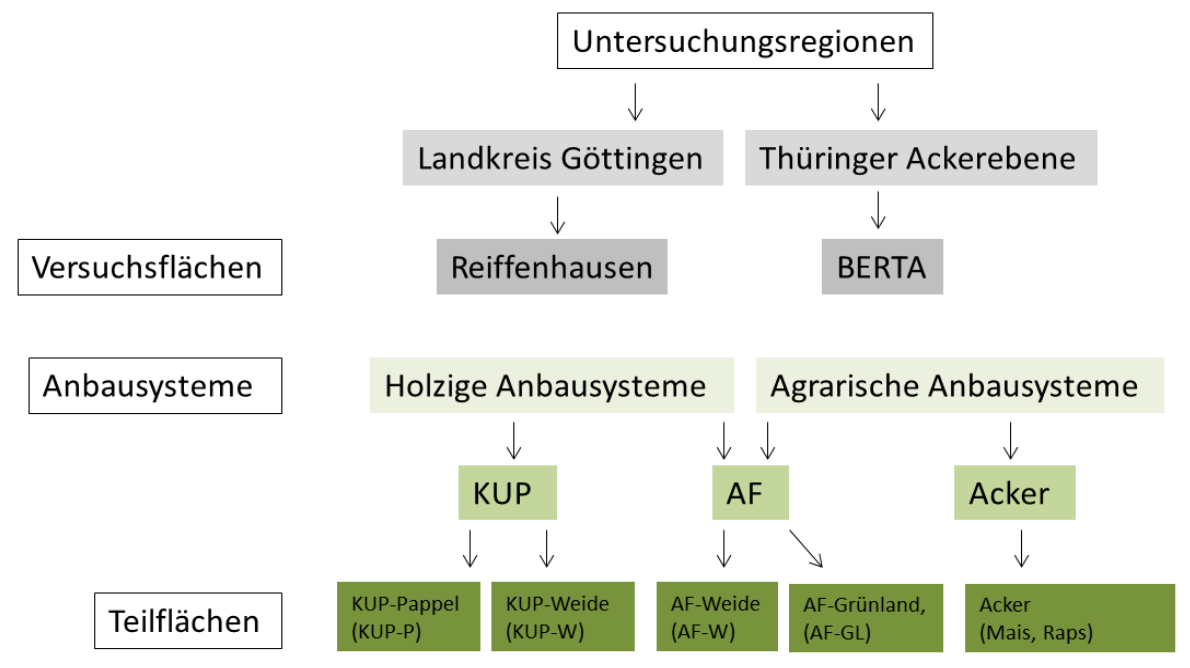

Abb. 2 Überblick zu den räumlichen Begriffen und Anbausystemen

\subsection{Zielsetzung}

Das BEST-Teilprojekt "Agroforst und Kurzumtriebsplantagen", bildet die Grundlage für die vorliegende Arbeit und untersucht innovative Anbausysteme zur Gewinnung von holziger Biomasse.

Die Hauptziele dieser Studie sind:

1. Entwicklung von regional angepassten Konzepten und innovativen Systemlösungen zur Produktion von Energieholz mittels Kurzumtriebsplantagen (KUP) und Agroforstsystemen (AF).

2. Bereitstellung von praxisrelevanten Versuchs- und Demonstrationsflächen mit KUP und AF in den beiden Bioenergie-Regionen "Göttinger Land" und "Thüringer Ackerebene".

3. Bodenökologische Charakterisierung der Versuchs- und Demonstrationsflächen.

4. Ableitung von Empfehlungen für die Praxis.

Zur Konkretisierung der Hauptziele wurden folgende Teilziele formuliert: 
- Bereitstellung, Analyse und Bewertung von Daten zu den klimatischen, geologischen, bodenphysikalischen und bodenchemischen Ausgangsbedingungen der Versuchsflächen in den beiden Bioenergie-Regionen.

- Analyse des Ernährungszustandes der schnellwachsenden Gehölze.

- Erfolgskontrolle durch Erfassung der Anwuchs- und Ertragsentwicklung.

- Vergleichende Bestimmung der Laubproduktion, des Laubumsatzes und der Nährstofffreisetzung für die eingesetzten schnellwachsenden Gehölze.

- Abschätzungen zu Vorräten und Flüssen des initialen Stickstoffkreislaufes.

- Integrierte Bewertung der Versuchsflächen hinsichtlich ihrer Eignung für den Anbau von KUP und AF.

\section{Material und Methoden}

Teile des Kapitels Material und Methoden wurden in einer Fachzeitschrift, einem Buchbeitrag und in Tagungsbänden veröffentlicht. Ausführliche Informationen zur Standortcharakteristik der Untersuchungsregionen und Versuchsflächen können den Beiträgen Hartmann et al. (2014) und Hartmann u. Lamersdorf (2014a) entnommen werden. Die Aufnahmemethodik zur Ertragsschätzung der holzigen Biomasse finden sich in Hartmann et al. (2013) und Hartmann et al. (2014). Informationen zur stofflichen Charakterisierung der holzigen Anbausysteme sind im Buchbeitrag Hartmann u. Lamersdorf (2014a) niedergelegt.

Statistische Besonderheiten finden sich stets am Ende der einzelnen Teilkapitel, grundlegend wurden jedoch folgende Arbeitsschritte in Abhängigkeit der Fragestellung durchgeführt:

- die Bearbeitung der Rohdaten sowie die graphische Aufbereitung der Ergebnisse erfolgte mit Excel Version 14.0.

- Berechnung des arithmetischen oder gewichteten Mittels und der Standardabweichung.

- Um signifikante Unterschiede zwischen den Mittelwerten zweier unabhängiger Stichproben zu ermitteln, wurde ein T-Test bei unabhängigen Stichproben durchgeführt. Als Voraussetzung zur Anwendung dieses Signifikanztestes müssen normalverteilte, metrische Daten vorliegen. Die Normalverteilung der Datensätze wurde mittels Kolmogorov-SmirnovTest bzw. Shapiro-Wilk-Test getestet. Signifikante Unterschiede ergaben sich stets, wenn die Irrtumswahrscheinlichkeit $p \leq 0,05$ ausfiel. Signifikante Unterschiede wurden unter Verwendung gleicher Symbole gekennzeichnet.

- Um signifikante Unterschiede zwischen den Mittelwerten bei mehr als zwei unabhängigen Stichproben zu bestimmen, wurde eine Varianzanalyse (einfaktorielle ANOVA) durchgeführt. 
Als Voraussetzung zur Anwendung der einfaktoriellen ANOVA müssen die abhängigen Daten mindestens ordinalskaliert und normalverteilt vorliegen. Die Normalverteilung der Datensätze wurde mittels Kolmogorov-Smirnov-Test bzw. Shapiro-Wilk-Test getestet. Signifikante Unterschiede ergaben sich stets, wenn die Irrtumswahrscheinlichkeit $p \leq 0,05$ ausfiel. Um genauere Aussagen zur Ungleichheit der Mittelwerte treffen zu können, wurden Post-Hoc-Mehrfachvergleiche berechnet. Bei Varianzheterogenität wurde der Tamhane-T2Test bzw. bei Varianzhomogenität der Tukey-Test verwendet. Signifikante Unterschiede wurden unter Verwendung gleicher Symbole gekennzeichnet.

- Im Falle nicht normalverteilter Variablen wurden Kruskal-Wallis-Tests berechnet, um signifikante Unterschiede zwischen den Mittelwerten bei mehr als zwei unabhängigen Stichproben zu ermitteln. Im Anschluss an diese, wurde der Mann-Whitney-Test durchgeführt. Es wurde darauf geachtet, dass das Signifikanzniveau entsprechend der Anzahl der Paarvergleiche geändert wurde (Schwarz et al. 2010). Signifikante Unterschiede wurden unter Verwendung gleicher Symbole gekennzeichnet.

- Mögliche Zusammenhänge zwischen zwei Stichproben wurden mit der bivariaten Korrelationsanalyse, mit einer Irrtumswahrscheinlichkeit von $p \leq 0,05$, ermittelt. Zuvor wurden die metrischen Daten auf ihre Normalverteilung mit dem Kolmogorov-Smirnov-Test bzw. Shapiro-Wilk-Test geprüft. Im Falle normalverteilter Daten wurde eine Korrelationsanalyse nach Pearson, bei nicht normalverteilten Daten eine Korrelationsanalyse nach Spearman berechnet.

- Alle statistischen Tests und Berechnungen wurden mit dem Programm SPSS Version 21.0 durchgeführt.

\subsection{Standortcharakteristik}

\subsubsection{Untersuchungsregionen}

\subsubsection{Räumliche Charakterisierung}

Der Landkreis Göttingen (Ldk. Gö.) mit der Versuchsfläche Reiffenhausen liegt im oberen Leinebergland und nimmt mit einer Fläche von $1.117 \mathrm{~km}^{2}$ den äußersten Süden Niedersachsens ein. Mit einer Höhenlage zwischen $110 \mathrm{~m}$ ü. NN (Weserwiesen bei Bursfelde) und $581 \mathrm{~m}$ ü. NN (Haferberg im Kaufunger Wald) weist der Landkreis eine deutliche vertikale Differenzierung auf. Die Landschaft um Göttingen ist kleinräumig gegliedert und mit ihren Wechsel aus Wald-, Grünland- und Ackerflächen strukturreich (Abb. 3). 


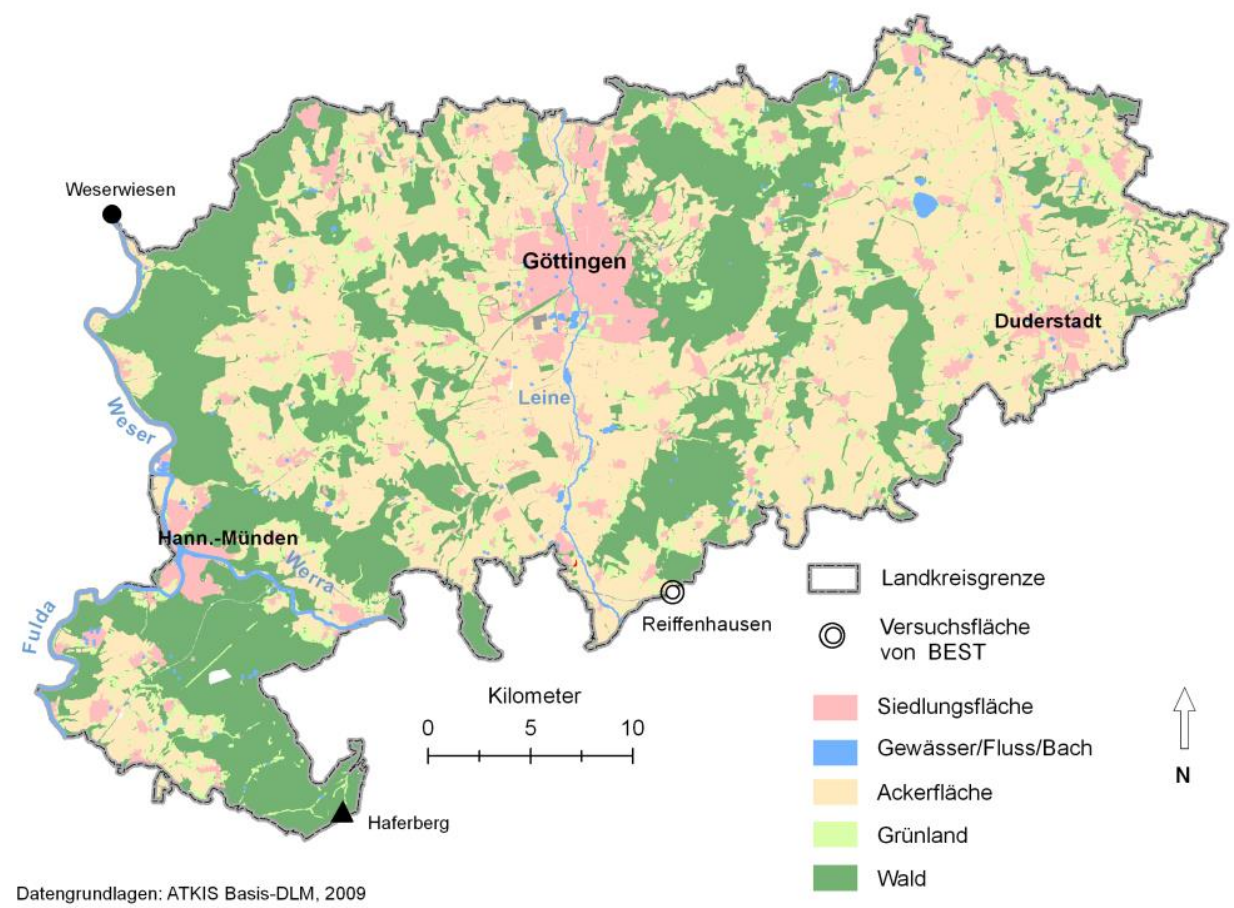

Abb. 3 Räumliche Struktur des Landkreises Göttingen mit Lage der Versuchsfläche

Die "Thüringer Ackerebene", mit der Versuchsfläche BERTA, liegt im zentralen Bereich des Thüringer Beckens und erstreckt sich über 35 Gemeinden der drei Landkreise Unstrut-Hainich, Sömmerda und Gotha und umfasst rund $556 \mathrm{~km}^{2}$ (AdV - Arbeitsgemeinschaft der Vermessungsverwaltungen der Länder der Bundesrepublik Deutschland 2003).

Das Landschaftsbild wird von zahlreichen großen Ackerschlägen dominiert (Abb. 4), nur $4 \%$ der Fläche sind mit Wald bestanden (Tabelle 1). Diese beschränken sich im Wesentlichen auf die Höhenlagen der Fahner Höhe und des Hainichs. 


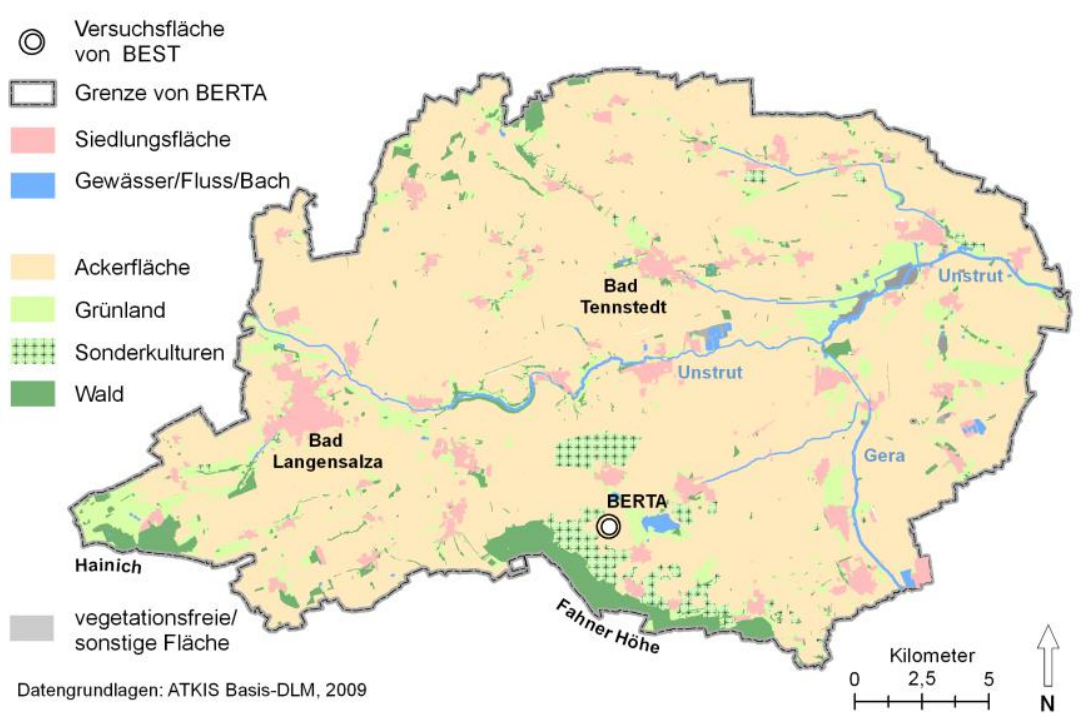

Abb. 4 Räumliche Struktur der Thüringer Ackerebene mit Lage der Versuchsfläche

Tabelle 1 Standortsdaten der Untersuchungsregionen: Landkreis Göttingen (Ldk. Gö.) und Thüringer Ackerebene

\begin{tabular}{|c|c|c|}
\hline & Ldk. Gö. & Thüringer Ackerebene \\
\hline Siedlungsfläche* [\%] & 18,6 & 10,7 \\
\hline Waldfläche* [\%] & 34,1 & 4,3 \\
\hline Landwirtschaftliche Fläche* [\%] & 54,4 & 86,2 \\
\hline Bevölkerungsdichte^ ${ }^{\wedge}$ Einwohner km ${ }^{-2}$ ] & 231 & 92 \\
\hline Mittlerer jährlicher Niederschlag (1991-2010) ${ }^{\dagger}[\mathrm{mm}]$ & 642 & 549 \\
\hline Mittlerer jährlicher Niederschlag Mai-Sept. $(1991-2010)^{\dagger}[\mathrm{mm}]$ & 312 & 289 \\
\hline Temperaturmittelwert $(1991-2010)^{\dagger}\left[{ }^{\circ} \mathrm{C}\right]$ & 9,2 & 9,4 \\
\hline
\end{tabular}

\subsubsection{Geologie}

Geomorphologisch gliedert sich der Ldk. Gö. in den zentralen Leinetalgraben, der im Osten von der Eichsfeldscholle und im Westen von der Sollingscholle flankiert wird (Arp et al. 2011b, Stille u. Lotze 1933). Im Graben waren die Sedimentgesteine des Keupers und Unteren Juras vor Abtrag geschützt (Arp et al. 2011b, Wunderlich 1964) und wurden von quartären Lockersedimenten vielfach überprägt. Die im Osten und Westen anschließenden Eichsfeld- bzw. Sollingscholle sind überwiegend aus den triassischen Gesteinsschichten des Buntsandsteins und Muschelkalks aufgebaut (Vollbrecht u. Tanner 2011), die die für Südniedersachsen typische Bruchschollentektonik aufweisen. Das Gebiet um Göttingen wurde nie von Gletschervorstößen erreicht (Heunisch et al. 2007, Seedorf u. Meyer 
1992). Die Versuchsfläche ist demzufolge periglazial z.B. durch Fließerden und Lössablagerungen geprägt.

Sie liegt am östlichen Ende der Eichsfeldscholle in unmittelbarer Nachbarschaft zum Leinetalgraben (Arp et al. 2011a). Die Störungen der geologischen Schichten sind in diesem Gebiet aufgrund der tertiären Tektonik im Graben und dessen Randzonen stark ausgeprägt. Auf der Fläche finden sich im Norden und Nordosten Ablagerungen der Solling-Folge des Mittleren Buntsandsteins (smS), die hauptsächlich aus Sandsteinen, aber auch Schluff- und Tonsteinen aufgebaut sind (LBEG - Landesamt für Bergbau Energie und Geologie 2012). Im Südwesten finden sich tonreiche Ablagerungen des Oberen Buntsandsteins (Röt-Formation). Beide Bereiche weisen im Oberboden eine deutliche Schluffkomponente auf, die auf den Lösseinfluss hinweist.

Die Versuchsfläche in der "Thüringer Ackerebene" befindet sich im zentralen Bereich des Thüringer Beckens, dem so genannten "Keuperbecken Innerthüringens". Dieses ist aus Ablagerungen des Unteren und Mittleren Keupers aufgebaut (Ernst u. Weigel 1992, Sedlacek 2002, Seidel u. Unger 1995). Abgelagert wurden stark differenzierte Sedimentgesteine wie Sandsteine, Tonsteine, Mergel, Anhydrit und Kohle (Neumann 1883, Seidel 2003). Die Gesteine des Keupers wurden durch quartäre Lockersedimente überlagert. Dabei handelt es sich entweder um äolisch abgelagertes, verspültes oder solifluidal verlagertes Material, dass sich zum großen Teil aus Gesteinsmaterial des KeuperUntergrundes und Lössbeimengungen zusammensetzt. Von den drei großen Inlandvereisungen erreichte lediglich das Elstereis den zentralen Bereich des Thüringer Beckens (Seidel 2003). Im nachfolgenden Saale- und Weichselglazial drangen die Eismassen nicht mehr bis in die Untersuchungsregion vor.

\subsubsection{Klima}

Zur Charakterisierung des Klimas der beiden Versuchsflächen wurden Tagesdaten des Deutschen Wetterdienstes (DWD) ausgewertet. Die zu den Versuchsflächen nächstgelegenen Klimastationen sind die DWD-Stationen Göttingen und Dachwig. Anstelle der üblichen 30-jährigen Monatswerte für Temperatur und Niederschlag wurden die langjährigen Monatswerte über die längste gemeinsame Messperiode beider Stationen 1991 - 2010 berechnet.

Die Jahresmitteltemperatur der DWD Station Göttingen beträgt demnach $9,2{ }^{\circ} \mathrm{C}$, die durchschnittliche jährliche Niederschlagsmenge liegt bei $642 \mathrm{~mm}$, in der Vegetationsperiode von Mai bis September bei $312 \mathrm{~mm}$ (Tabelle 1).

In der Vegetationsperiode von Mai bis September 2011 wurden im Ldk. Gö. Niederschläge von nur $247 \mathrm{~mm}$ gemessen, im Vergleich zum langjährigen Mittel von $312 \mathrm{~mm}$ (Tabelle 1). Das Etablierungsjahr (2011) ist durch eine ausgeprägte Frühjahrstrockenheit charakterisiert (Abb. 5). Besonders in den Monaten Februar bis Mai fielen teils deutlich geringere Niederschläge. In diesem 
Zeitraum fielen nur etwa $40 \%$ des langjährigen Niederschlages. Besonders ausgeprägt war die Trockenperiode im Monat Mai, der für die Entwicklung der Vegetation im Frühjahr entscheidend sein kann.

Darüber hinaus wichen im Jahr 2011 auch die Monate Juli und November vom langjährigen Mittel ab, letzterer liegt mit weniger als $1 \mathrm{~mm}$ Niederschlag in Göttingen deutlich unter dem langjährigen Minimum.

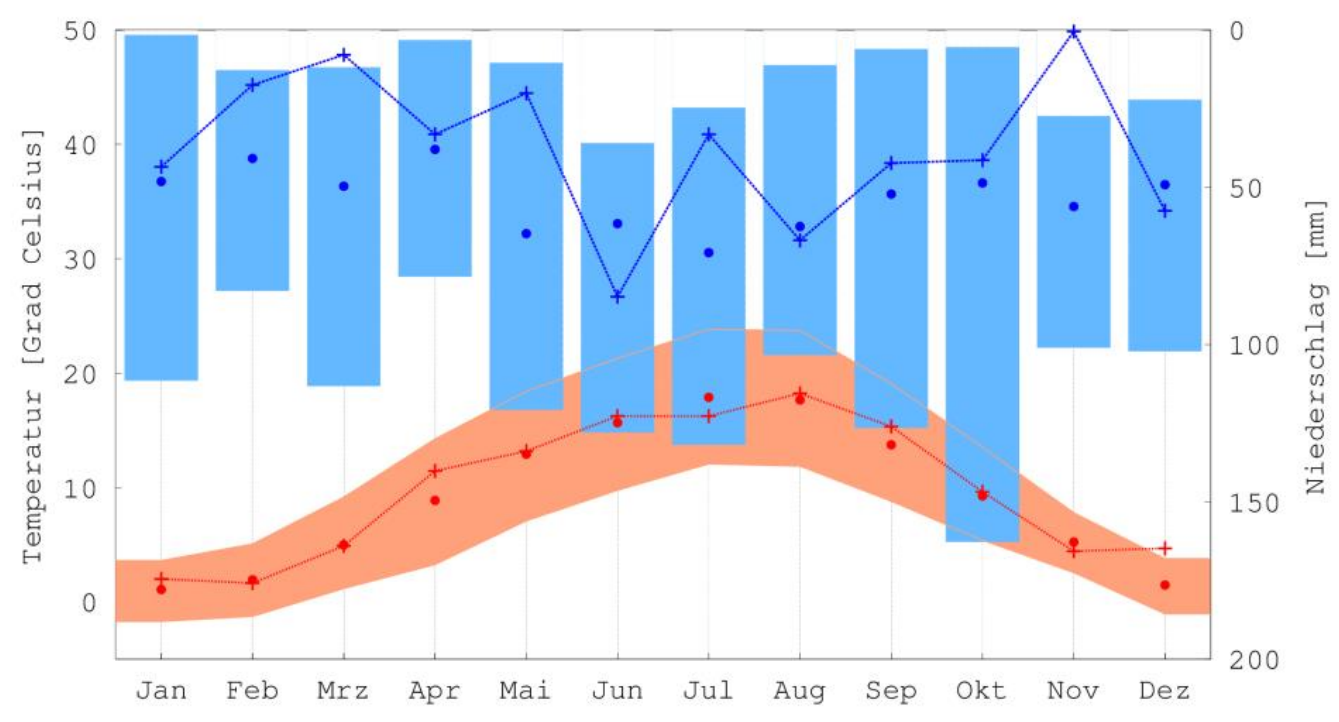

Abb. 5 Monatswerte von Temperatur (rot) und Niederschlag (blau) der DWD-Station Göttingen. Punkte zeigen langjährige Monatswerte (Periode 1991-2010) mit den absoluten Minima und Maxima als Schattierungen. Die Linien mit Kreuzen repräsentieren jeweils Temperatur und Niederschlag für 2011, das Etablierungsjahr der Kurzumtriebsplantagen und Agroforstsysteme

Die zu den Versuchsflächen der "Thüringer Ackerebene" nächstgelegene DWD-Station ist Dachwig. Das Klima ist mit einer Jahresmitteltemperatur von $9,4^{\circ} \mathrm{C}$ etwas wärmer und mit einer durchschnittlichen jährlichen Niederschlagsmenge von 549 mm trockner als im Ldk. Gö. Während der Vegetationsperiode von Mai bis September fielen in der "Thüringer Ackerebene" im langjährigen Mittel 289 mm Niederschlag (Tabelle 1). Das Etablierungsjahr 2011 war auch in dieser Untersuchungsregion durch eine ausgeprägte Frühjahrstrockenheit gekennzeichnet. In der Vegetationsperiode von Mai bis September 2011 wurden Niederschläge von nur 214 mm gemessen, im Vergleich zum langjährigen Mittel mit $289 \mathrm{~mm}$. Auch hier weichen die Niederschläge in den Monaten Mai, Juli und November besonders deutlich von den langjährigen Mittelwerten ab (Abb. 6). 


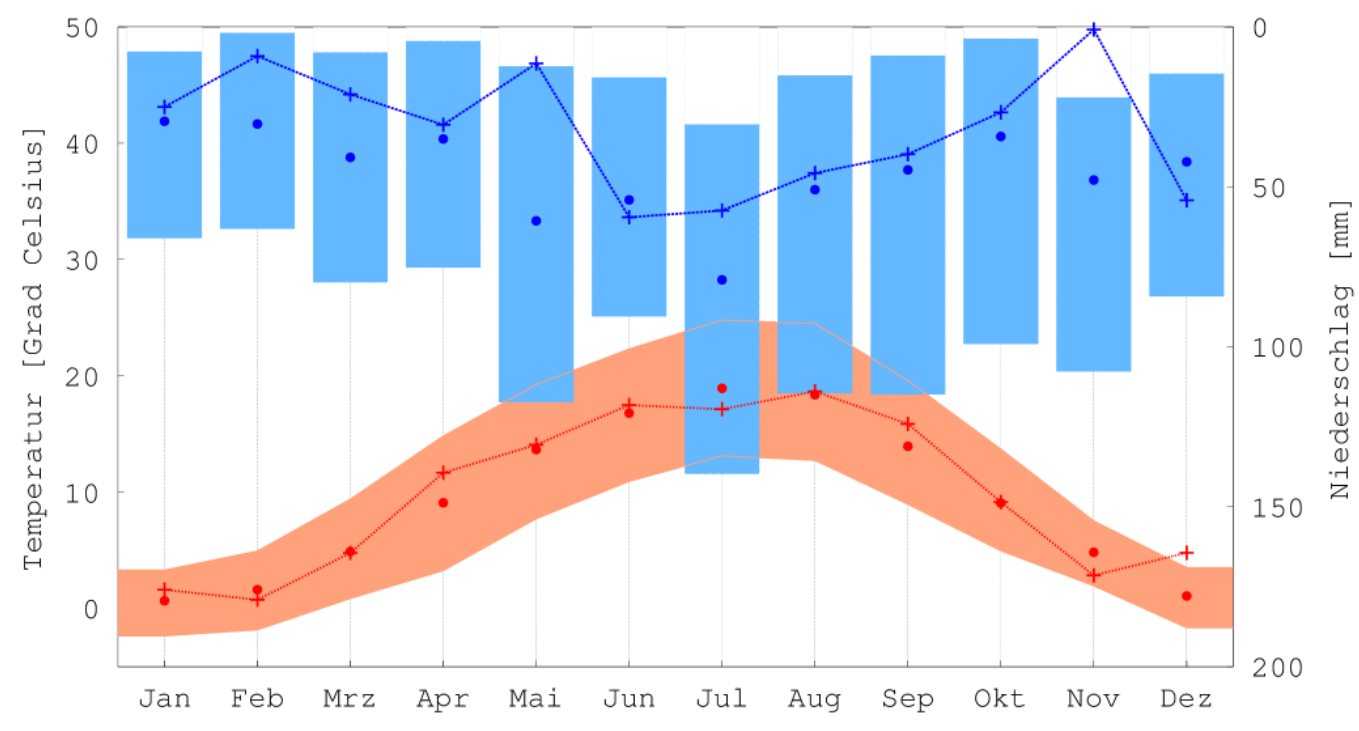

Abb. 6 Monatswerte von Temperatur (rot) und Niederschlag (blau) der DWD-Station Dachwig. Punkte zeigen langjährige Monatswerte (Periode 1991-2010) mit den absoluten Minima und Maxima als Schattierungen. Die Linien mit Kreuzen repräsentieren jeweils Temperatur und Niederschlag für 2011, das Etablierungsjahr der Kurzumtriebsplantagen und Agroforstsysteme

\subsubsection{Versuchsflächen}

\subsubsection{Flächendesign und Bewirtschaftung}

Im Ldk. Gö. wurde die ca. 1,7 ha große Versuchsfläche Reiffenhausen in der Nähe der Gemeinde Reiffenhausen etabliert. Die Versuchsfläche Reiffenhausen mit einer Ackerzahl von 35-57 ist umgeben von konventionell bewirtschafteten Ackerbereichen und dem Biotopverbund "Grünes Band" (Abb. 7, links). Sie besteht aus zwei KUP (Pappel 0,4 ha, Weide 0,6 ha) und einem AF (0,7 ha). An die Versuchsfläche schließt sich im Nordwesten eine Ackerfläche an, die 2010/2011 mit Wintergerste und 2011/12 mit Winterraps bestellt und als Referenzfläche herangezogen wurde.

Da die Arbeiten im Forschungsprojekt erst im November 2010 aufgenommen werden konnten, war die Wintergerste bereits ausgesät. Dies verhinderte die Ausbringung eines Totalherbizids mit anschließendem Pflügen, ein Standardverfahren, das für gewöhnlich in der Literatur für die Anlage von KUP empfohlen wird. Stattdessen wurde die Fläche im Februar 2011 gegrubbert. Nach der Pflanzung der Stecklinge wurden verschiedene Maßnahmen zum Erhalt und Wachstum der Bestände ergriffen. Dazu zählten die Applikation eines Vorauflaufmittels unmittelbar nach der Pflanzung sowie die mechanische Beikrautregulierung durch Hacken und Mähen. Ferner wurden im Frühjahr 2012 die Pflanzausfälle durch Steckruten ( $80 \mathrm{~cm}$ Länge) ersetzt. 


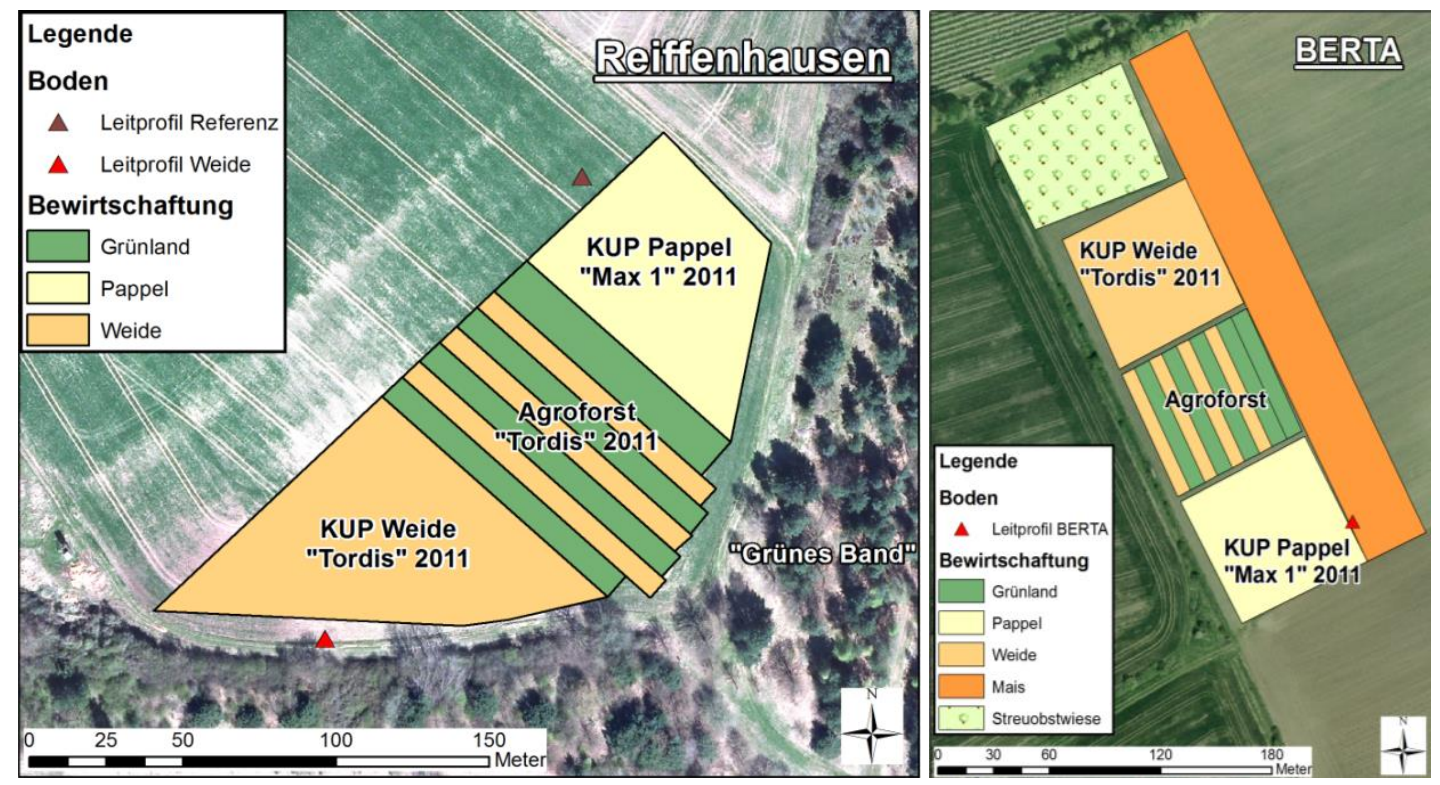

Abb. 7 Flächendesign der Versuchsflächen Reiffenhausen (links) und BERTA (rechts) mit Angaben zum Jahr der Bestandsbegründung und der verwendeten Klone. Lage der Leitprofile. Datengrundlage: Reiffenhausen: Digitales Orthophoto 2010, Quelle: Auszug aus den Geobasisdaten der Niedersächsischen Vermessungs- und Katasterverwaltung, (C) 2011 Landesamt für Geoinformation und Landentwicklung Niedersachsen (LGLN); BERTA: Digitales Orthophoto 2008, Quelle: (C) GeoBaisDE / TLVermGeo

Die Versuchsfläche BERTA, mit einer durchschnittlichen Ackerzahl von 75, ist ca. 1,8 ha groß und besteht aus insgesamt 3 neuangelegten Teilflächen: zwei KUP (jeweils 0,6 ha) und ein AF (0,6 ha). Angrenzend an diese Teilflächen wurde zusätzlich eine konventionelle Ackerfläche mit Mais ( 1 ha) angelegt, die als Referenzfläche dient (Abb. 7, rechts). Um die Bestände vor Unkraut zu schützen, wurden auf der Fläche chemische (Applikation Nachauflaufmittel) und mechanische Maßnahmen (Hacken, Mähen und Fräsen) ergriffen. Ferner wurden ausgefallene Stecklinge im Frühjahr 2012 durch Ruten (80 cm Länge) ersetzt. Vor der Anpflanzung der schnellwachsenden Gehölze war die neuangelegte Fläche mit Winterweizen und Sommergerste bestellt worden.

Die Bestandsbegründung mit Pappeln bzw. Weiden auf den Flächen BERTA und Reiffenhausen erfolgte manuell mit Pflanzschnur und Steckeisen von Mitte März bis Mitte April 2011. Die Stecklinge wiesen dabei eine Länge von ca. $20 \mathrm{~cm}$ auf und wurden ebenerdig gepflanzt. Dabei wurde auf einen guten Bodenschluss geachtet.

Auf beiden Flächen wurde der Pappelklon "Max 1" (P. nigra x P. maximowiczii) und der Weidenklon "Tordis" ((Salix viminalis $x$ Salix Schwerinii) $\mathrm{x}$ Salix viminalis)) verwendet.

Für die KUP-Weide und AF-Weide wurden die Stecklinge in Doppelreihenverbänden angepflanzt. Der Abstand zwischen den Doppelreihen betrug 1,5 m. Der Baumabstand innerhalb einer Reihe variierte in Abhängigkeit der eingesetzten Gehölze. Bei Pappeln betrug dieser $1 \mathrm{~m}$ und bei Weiden 0,75 m. Bei der Pappel ist die Pflanzdichte mit ca. 8.890 Stecklingen pro ha geringer als bei der Weide mit ungefähr 11.850 Stecklingen pro ha (Tabelle 2). Für die Teilfläche AF-Weide ergab sich eine durchschnittliche Bestockung von 5.330 Weiden pro ha. 
Tabelle 2 Übersicht zum Flächendesign der neu angelegten Versuchsflächen Reiffenhausen und BERTA

\begin{tabular}{|c|c|c|c|c|c|c|c|}
\hline \multirow[t]{2}{*}{ Versuchsfläche } & \multirow[t]{2}{*}{ Teilfläche } & \multirow[t]{2}{*}{ Anlagejahr } & \multirow[t]{2}{*}{$A Z^{*}$} & \multirow[t]{2}{*}{ Klon } & \multirow{2}{*}{$\begin{array}{l}\text { Größe } \\
\text { [ha] }\end{array}$} & \multirow{2}{*}{$\begin{array}{l}\text { Pflanzverband } \\
{[\mathrm{m}]}\end{array}$} & \multirow{2}{*}{$\begin{array}{l}\text { Pflanzzahl } \\
\text { [ha] }\end{array}$} \\
\hline & & & & & & & \\
\hline \multirow[t]{3}{*}{ Reiffenhausen } & KUP-Pappel & 2011 & $35-57$ & $\operatorname{Max} 1$ & 0,4 & $0,75 / 1,5 / 1,0$ & 8.890 \\
\hline & KUP-Weide & 2011 & $35-57$ & Tordis & 0,6 & $0,75 / 1,5 / 0,75$ & 11.850 \\
\hline & AF-Weide & 2011 & $35-57$ & Tordis & 0,7 & $0,75 / 1,5 / 0,75$ & 5.330 \\
\hline \multirow[t]{3}{*}{ BERTA } & KUP-Pappel & 2011 & 75 & $\operatorname{Max} 1$ & 0,6 & $0,75 / 1,5 / 1,0$ & 8.890 \\
\hline & KUP-Weide & 2011 & 75 & Tordis & 0,6 & $0,75 / 1,5 / 0,75$ & 11.850 \\
\hline & AF-Weide & 2011 & 75 & Tordis & 0,6 & $0,75 / 1,5 / 0,75$ & 5.330 \\
\hline
\end{tabular}

${ }^{*} \mathrm{AZ}=\mathrm{Ackerzahl}$

\subsubsection{Leitprofile}

Im August 2011 wurden auf beiden Versuchsflächen Leitprofile bis in ca. $2 \mathrm{~m}$ Tiefe angelegt. Insgesamt wurden 3 Profile gegraben. In Reiffenhausen wurden wegen der größeren räumlichen Heterogenität des geologischen Ausgangsmaterials zwei Bodenprofile (Reiffenhausen Referenz $=\mathrm{RR}$, Reiffenhausen Weide = RW) aufgegraben (Abb. 7). In der "Thüringer Ackerebene" wurde ein Profil (BERTA = B) angelegt, wobei die genaue Lokalisierung und Bezeichnung aus der Abb. 7 entnommen werden kann.

Die Bodentypen der Flächen wurden nach der Bodenkundlichen Kartieranleitung KA5 (Ad-HocArbeitsgruppe Boden 2005) angesprochen.

Aus allen drei Profilen wurden Bodenproben aus einer Tiefe von 0-10 cm, $10-30 \mathrm{~cm}, 30-60 \mathrm{~cm}, 60-$ $90 \mathrm{~cm}$ in dreifacher Wiederholung entnommen.

Die Bodenproben wurden im Trockenschrank bei $40^{\circ} \mathrm{C}$ getrocknet und anschließend durch ein Sieb mit der Maschenweite 2 mm gesiebt. Die Bestimmung der Korngrößenzusammensetzung erfolgte am getrockneten Mineralboden $(<2 \mathrm{~mm})$ durch Siebung und Sedimentation (Gutachterausschuss Forstliche Analytik 2005). Der Skelettanteil (> $2 \mathrm{~mm}$ ) am Gesamtboden wurde an der Profilwand im Gelände nach Abb. 3.5.1 (Blume et al. 2011) geschätzt.

Die Bestimmung der Trockenraumdichte (TRD) erfolgte nach der Stechzylinder-Methode (Gutachterausschuss Forstliche Analytik 2005). Pro Tiefenstufe wurden 4 Stechzylinder von $100 \mathrm{~cm}^{3}$ entnommen.

Die Kohlenstoff- und Nährstoffgehalte wurden anhand der tiefenstufenabhängig gewonnenen Leitprofilbodenproben bestimmt. Eine Teilmenge dieser Bodenproben, die zuvor fein gemahlen worden war (Fa. Retsch-PM 4000), wurde auf ihren Gesamtkohlenstoff $\left(C_{t}\right)$ und -stickstoffgehalt $\left(N_{t}\right)$ hin analysiert. Die Gehalte an $\mathrm{C}_{\mathrm{t}}$ und $\mathrm{N}_{\mathrm{t}}$ wurden mittels oxidativer Verbrennung am C-N-Analysator (CHN-O-Rapide, VarioEL, Fa. Elementar) gemessen. Die Bestimmung des organischen Kohlenstoffs $\left(C_{\text {org }}\right)$ erfolgte durch Messung des Kohlenstoffs vor und nach Zerstörung der Carbonate mit Salzsäure. Der Carbonatgehalt (Dolomit, Calcit) wurde aus der Differenz zwischen $C_{t}$ und $C_{\text {org }}$ ermittelt. 
Der Gesamtgehalt an Phosphor $\left(\mathrm{P}_{\mathrm{t}}\right)$ in den Bodenproben wurde nach $\mathrm{HNO}_{3}$-Druckaufschluss mittels ICP-AES (Inductively Coupled Plasma-Atomic Emission Spectrometer, Fa. Thermo Fisher) bestimmt.

Die Analyse des pH-Wertes erfolgte in bidestilliertem Wasser mit einem pH-Meter (Gutachterausschuss Forstliche Analytik 2005).

Die Bestimmung der potenziellen Kationenaustauschkapazität $\left(\mathrm{KAK}_{\mathrm{pot}}\right)$ erfolgte durch Austausch der adsorbierten Kationen mittels Bariumchlorid-Lösung bei pH 8,1 (Gutachterausschuss Forstliche Analytik 2005).

Die flächenbezogene Akkumulation (Vorrat) an Nährstoffen (t ha ${ }^{-1}$ ) wurde durch Multiplikation der Bodenmenge, die unter Berücksichtigung der Trockenraumdichte, des Skelett- und Carbonatgehaltes ermittelt wurde, mit den Stoffkonzentrationen berechnet und nach den Klassen in Tabelle 3 bewertet. Die Bestimmung der Bodenmenge erfolgte für die Tiefenstufen 0-10 cm, 10-30 cm, 30$60 \mathrm{~cm}, 60-90 \mathrm{~cm}$.

Tabelle 3 Bewertungsklassen für die Vorräte an Gesamtstickstoff $\left(N_{t}\right)$, organischen Kohlenstoff $\left(C_{\text {org }}\right)$, Kalium $(\mathrm{K})$, Calcium (Ca), Magnesium ( $\mathrm{Mg}$ ) und Gesamtphosphor $\left(\mathrm{P}_{\mathrm{t}}\right)$ und Bewertungsklassen der potenziellen Kationenaustauschkapazität (KAK ${ }_{\text {pot }}$ ) im effektiven Wurzelraum

\begin{tabular}{llllllll}
\hline Bewertung & $\mathrm{N}_{\mathrm{t}}{ }^{*}$ & $\mathrm{C}_{\text {org }}{ }^{-1}$ & $\mathrm{~K}^{*}$ & $\mathrm{Ca}^{*}$ & $\mathrm{Mg}^{*}$ & $\mathrm{P}_{\mathrm{t}}{ }^{*}$ & $\mathrm{KAK}_{\text {pot }}{ }^{-1}$ \\
\cline { 2 - 7 } & {$\left[\mathrm{t} \mathrm{ha}^{-1}\right]$} & {$\left[\mathrm{t} \mathrm{ha}^{-1}\right]$} & {$\left[\mathrm{t} \mathrm{ha}^{-1}\right]$} & {$\left[\mathrm{t} \mathrm{ha}^{-1}\right]$} & {$\left[\mathrm{t} \mathrm{ha}^{-1}\right]$} & {$\left[\mathrm{t} \mathrm{ha}^{-1}\right]$} & {$\left[\mathrm{mmol}_{\mathrm{c}} \mathrm{kg}^{-1}\right]$} \\
\hline sehr gering & $<2,5$ & $<50$ & $<0,2$ & $<0,2$ & $<0,05$ & $<0,75$ & $<25$ \\
gering & $2,5-<5$ & $50-<100$ & $0,2-<0,4$ & $0,2-<0,4$ & $0,05-<0,1$ & $0,75-<1,5$ & $25-<50$ \\
mittel & $5-<10$ & $100-<200$ & $0,4-<1,2$ & $0,4-<4$ & $0,1-<1$ & $1,5-<3$ & $50-<400$ \\
hoch & $10-<20$ & $200-<400$ & $1,2-<1,6$ & $4-<8$ & $1-<2$ & $3-<7,5$ & $400-<800$ \\
sehr hoch & $\geq 20$ & $\geq 400$ & $\geq 1,6$ & $\geq 8$ & $\geq 2$ & $\geq 7,5$ & $\geq 800$ \\
\hline
\end{tabular}

*nach Arbeitskreis Standortskartierung 2003

\subsubsection{Feinkartierung}

Im Februar 2011 fand eine flächenhafte Kartierung des Bodens in Reiffenhausen und BERTA bis in ca. $1 \mathrm{~m}$ Tiefe mit dem Pürckhauer-Bohrer statt. Dabei wurden jeweils 9 Punkte pro Fläche beprobt (Abb. 8). Diese Kartierung diente vor allem dazu, Informationen zur räumlichen Verteilung der Nährstoffe zu erhalten und mit den Ergebnissen aus den Leitprofilen zu vergleichen. Die Bodentypen wurden nach der Bodenkundlichen Kartieranleitung KA5 (Ad-Hoc-Arbeitsgruppe Boden 2005) angesprochen. Die Entnahme von Bodenproben erfolgte aus einer Tiefe von $0-30 \mathrm{~cm}, 30-60 \mathrm{~cm}$ und $60-90 \mathrm{~cm}$ in dreifacher Wiederholung.

Die Bodenproben wurden im Trockenschrank bei $40{ }^{\circ} \mathrm{C}$ getrocknet und anschließend durch ein Sieb mit der Maschenweite $2 \mathrm{~mm}$ gesiebt. Die Bestimmung der Korngrößenzusammensetzung erfolgte am getrockneten Mineralboden $(<2 \mathrm{~mm})$ durch Siebung und Sedimentation (Gutachterausschuss Forstliche Analytik 2005).

Zur bodenchemischen Untersuchung wurden die gleichen Methoden wie in Kapitel 2.1.2.2 angewandt. 
Die flächenbezogene Akkumulation (Vorrat) an Nährstoffen ( $\mathrm{t} \mathrm{ha}^{-1}$ ) wurde durch Multiplikation der Bodenmenge, die unter Berücksichtigung einer mittleren Trockenraumdichte $\left(1,5 \mathrm{~g} \mathrm{~cm}^{-3}\right.$ für die Bodentiefe $0-60 \mathrm{~cm} \& 1,6 \mathrm{~g} \mathrm{~cm}^{-3}$ für die Bodentiefe $60-90 \mathrm{~cm}$ in Reiffenhausen sowie $1,4 \mathrm{~g} \mathrm{~cm}^{-3}$ für

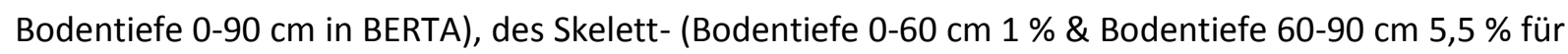
Reiffenhausen und Bodentriefe $0-30 \mathrm{~cm}: 5 \%$, Bodentiefe $30-90 \mathrm{~cm}: 50 \%$ für BERTA) und des Carbonatgehaltes ermittelt wurde, mit den Stoffkonzentrationen berechnet und nach den Klassen in Tabelle 3 bewertet. Die Bestimmung der Bodenmenge erfolgte für die Tiefenstufen 0-30 cm, 30$60 \mathrm{~cm}, 60-90 \mathrm{~cm}$.

Um die Nährstoffgehalte und Nährstoffvorräte zwischen den Leitprofilen und der Feinkartierung zu vergleichen, wurde ein T-Test durchgeführt. Zuvor wurden für die beiden Leitprofile in Reiffenhausen Mittelwerte für die Tiefenstufen 0-10 cm bzw. 10-30 cm berechnet. Um die beiden Tiefenstufen 0$10 \mathrm{~cm}$ und $10-30 \mathrm{~cm}$ aus dem Leitprofil mit der Tiefenstufe $0-30 \mathrm{~cm}$ aus der Feinkartierung zu vergleichen, wurde das gewichtete arithmetische Mittel (bei Nährstoffgehalten) bzw. die Summe (bei Nährstoffvorräten) berechnet. Anschließend wurde geprüft, ob der Mittelwert bzw. die Summe des Leitprofils (BERTA, Reiffenhausen) sich signifikant vom Flächenmittel (Nährstoffgehalte) bzw. von der Flächensumme (Nährstoffvorräte) der Feinkartierung unterscheiden.
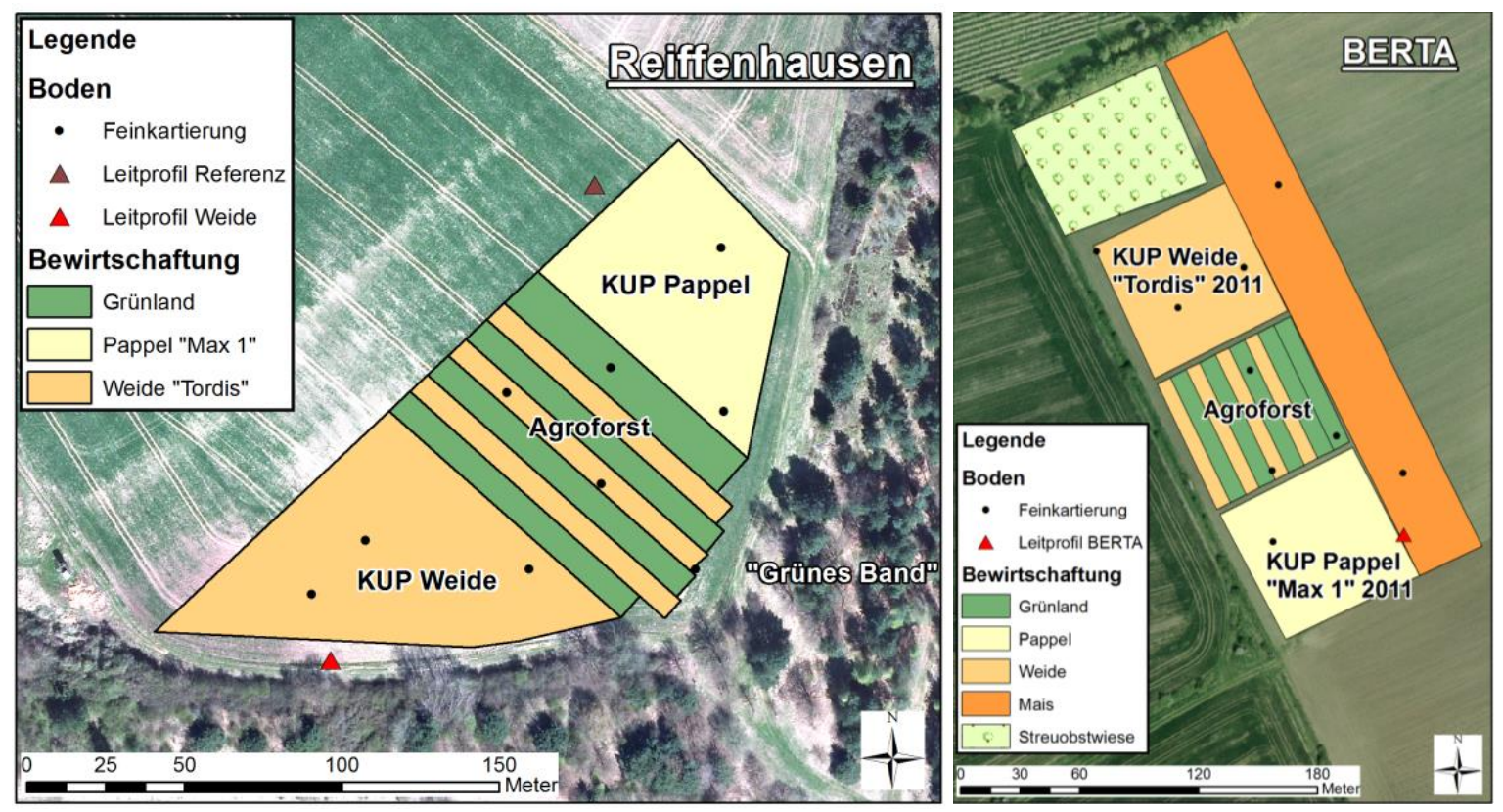

Abb. 8 Lage der Leitprofile und der Punkte für die Feinkartierung in Reiffenhausen (links) und BERTA (rechts). Datengrundlage: Reiffenhausen: Digitales Orthophoto 2010, Quelle: Auszug aus den Geobasisdaten der Niedersächsischen Vermessungs- und Katasterverwaltung, (C) 2011 Landesamt für Geoinformation und Landentwicklung Niedersachsen (LGLN), BERTA: Digitales Orthophoto 2008, Quelle: C GeoBaisDE / TLVermGeo

\section{$2.2 \mathrm{~N}$-Kreislauf, -Vorräte und -Flüsse}

Um Informationen über die N-Vorräte (u.a. Boden: extrahierbarer mineralischer Stickstoff, extrahierbarer organischer Stickstoff) und Flüsse (u.a. N-Nettomineralisation) und deren zeitlichen Verlauf zu erhalten, wurden Beprobungsbereiche auf den Teilflächen eingerichtet (Abb. 9). Bei der 
Auswahl der Bereiche wurde darauf geachtet, dass keine Fahrgassen und Vorgewende eingeschlossen wurden.

Ferner wurde bei der Einrichtung der Beprobungsbereiche in den holzigen Anbausystemen berücksichtigt, dass diese mit Bäumen bestanden sind und sich möglichst nah zur Klimastation und anderen Messeinrichtungen (Tensiometer, Gashauben) befinden. Die Entnahme von Bodenproben beschränkte sich in der Regel auf den Tiefenbereich 0-30 cm, da davon ausgegangen wurde, dass im betrachteten Zeitraum Veränderungen auf den Oberboden beschränkt bleiben würden.

Die Beprobungsbereiche auf den Teilflächen KUP-Pappel wiesen dabei eine Fläche von $117 \mathrm{~m}^{2}$ mit 104 Bäumen auf. Für KUP-Weide und AF-Weide wurden Beprobungsbereiche von $88 \mathrm{~m}^{2}$ mit ebenfalls 104 Bäumen eingerichtet. Auf der Referenzfläche Acker und auf dem Agroforst-Grünland wurden Beprobungsbereiche von ca. $25 \mathrm{~m}^{2}$ angelegt, auf denen regelmäßig Bodenproben entnommen wurden.
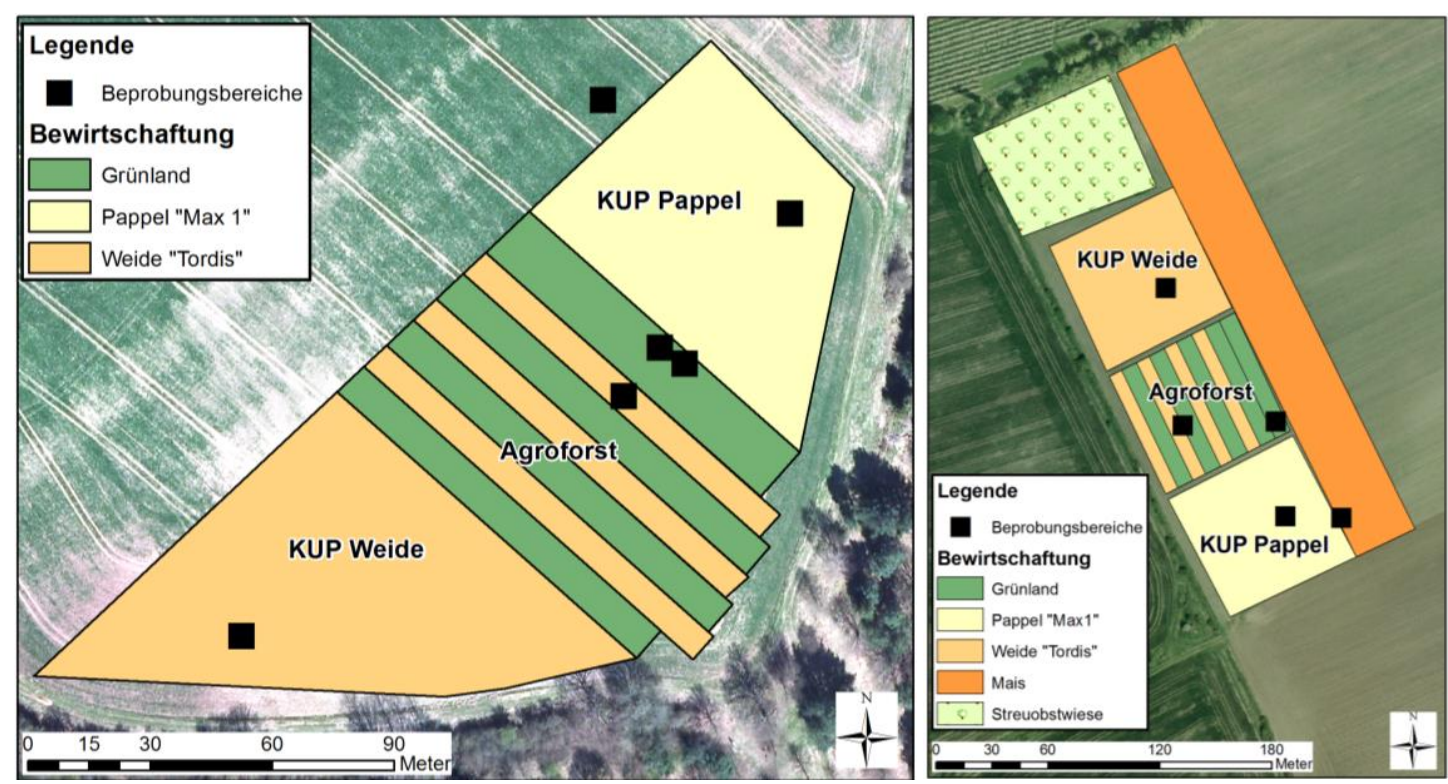

Abb. 9 Lokalisation der Beprobungsbereiche auf den Versuchsflächen Reiffenhausen (links) und BERTA (rechts). Datengrundlage: Reiffenhausen: Digitales Orthophoto 2010, Quelle: Auszug aus den Geobasisdaten der Niedersächsischen Vermessungs- und Katasterverwaltung, (C) 2011 Landesamt für Geoinformation und Landentwicklung Niedersachsen (LGLN), BERTA: Digitales Orthophoto 2008, Quelle: @ GeoBaisDE / TLVermGeo

\subsubsection{Extrahierbarer mineralischer und organischer Stickstoff}

Ab Oktober 2011 bis September 2012 wurden monatlich in dreifacher Wiederholung pro Teilfläche (KUP-Pappel, KUP-Weide, AF-Weide, AF-Grünland, Acker) Bodenproben aus 0-30 cm Tiefe in Reiffenhausen und BERTA aus den Beprobungsbereichen entnommen und analysiert. Dazu wurde ein Teil der im Gelände gewonnenen Bodenproben bis zur Verarbeitung kühl und dunkel bei maximal $4{ }^{\circ} \mathrm{C}$ gelagert.

Der extrahierbare Gesamtstickstoff- (eTN), Ammonium- (elN-NH 4 ) und Nitratgehalt (elN-NO durch Extraktion des Bodens mit 0,5 mol/L Kaliumsulfatlösung bestimmt. Dazu wurde der Boden mit der Kaliumsulfatlösung eine Stunde geschüttelt und anschließend filtriert. Die Bestimmung der Stickstoffgehalte der Extrakte, die mit destilliertem Wasser im Verhältnis 1:2 vorverdünnt wurden, 
erfolgte mittels Continuous-Flow-Injection-Colorimetry (Cenco/Skalar Instruments). Der extrahierbare mineralische Stickstoff (eIN) ergibt sich aus der Summe von eIN-NH 4 und eIN-NO Differenz zwischen eTN und eIN ergibt den extrahierbaren organischen Stickstoff (eON). Die flächenbezogene Akkumulation an Stickstoff $\left[\mathrm{kg}(\mathrm{N}) \mathrm{ha}^{-1}\right]$ wurde durch Multiplikation der Bodenmenge $\left[\mathrm{kg} \mathrm{ha}^{-1}\right]$, die unter Berücksichtigung der Trockenraumdichte, des Skelett- und Kalkgehaltes ermittelt wurde, mit der Stickstoffkonzentration $\left[\mathrm{mg}(\mathrm{N}) \mathrm{kg}^{-1}\right]$ berechnet. Die Bestimmung der Bodenmenge erfolgte für die Tiefenstufe 0-30 cm.

Um signifikante Unterschiede in den mittleren monatlichen eIN- und eON- Mengen zwischen den verschiedenen Teilflächen zu bestimmen, wurde die Varianzanalyse bzw. der Kruskal-Wallis-Test durchgeführt.

\subsubsection{Stickstoff-Nettomineralisation}

Die Stickstoff-Nettomineralisation, also die Änderung der Ammonium- und Nitrat-Gehalte in Abhängigkeit von der Zeit, wurde mit Hilfe der Buried Bag Methode von März 2012 bis Oktober 2012 bestimmt (Eno 1960, Kleber et al. 2000, Tracy u. Frank 1998). Dazu wurden pro Teilfläche (KUPPappel, KUP-Weide, Acker, AF-Grünland, AF-Weide) vier luftdurchlässige, jedoch wasserdichte Frühstücksbeutel (z.B. Firma Toppits) mit Boden befüllt, der aus den Tiefenbereich $0-30 \mathrm{~cm}$ gewonnen wurde. Zusätzlich wurden drei weitere Proben aus dem gleichen Tiefenbereich zur Bestimmung der anfänglichen Ammonium- und Nitratgehalte entnommen (entspricht eIN in der Vegetationsperiode, vgl. Kapitel 2.2.1). Der Boden wurde kühl (maximal $4{ }^{\circ} \mathrm{C}$ ) und dunkel bis zur Analyse gelagert. Die Frühstücksbeutel wurden in $15 \mathrm{~cm}$ Bodentiefe für ca. 6 Wochen eingebracht (Gasche et al. 2002). Anschließend wurden die Beutel wieder entnommen. Der Wassergehalt im Beutel blieb über die gesamte Inkubationsperiode konstant, wodurch ein geschlossenes System geschaffen worden war, aus dem es weder zu N-Verlusten durch pflanzliche Aufnahme oder Auswaschung noch zu N-Einträgen durch Niederschläge kommen konnte (Hein 2003).

Bis zur Analyse wurden auch diese Proben kühl (maximal $4{ }^{\circ} \mathrm{C}$ ) und dunkel gelagert. Die Bestimmung der extrahierbaren Ammonium- und Nitratgehalte $\left[\mathrm{mg}(\mathrm{N}) \mathrm{g}^{-1}\right.$ ] erfolgte mittels Continuous-FlowAnalyse (vgl. Kapitel 2.2.1).

Die N-Nettomineralisationsrate wurde nach folgender Formel berechnet:

$N M=\frac{(A e+N e)-(A a+N a)}{t}$

wobei $N M$ die N-Nettomineralisationsrate $\left[\mathrm{mg} \mathrm{kg}^{-1} \mathrm{~d}^{-1}\right]$, Ae der extrahierbare Ammoniumgehalt am Ende der Inkubation [mg (N) $\mathrm{kg}^{-1}$ ], Ne der extrahierbare Nitratgehalt am Ende der Inkubation [mg (N) $\mathrm{kg}^{-1}$ ], Aa der extrahierbare Ammoniumgehalt zu Beginn der Inkubation [mg (N) $\mathrm{kg}^{-1}$ ], Na der extrahierbare Nitratgehalt zu Beginn der Inkubation $\left[\mathrm{mg}(\mathrm{N}) \mathrm{kg}^{-1}\right]$ und t die Inkubationsdauer in Tagen 
[d] darstellt. Die Gehalte wurden dann mit der Bodenmenge (0-30 $\mathrm{cm}$ Tiefe, $\mathrm{kg} \mathrm{ha}^{-1}$ ) multipliziert, gemittelt und auf einen Zeitraum von 30 Tage (mittlere Monatsmengen) bezogen. Fehlende Werte z.B. in Reiffenhausen für Acker im Juli/August 2012 und in BERTA für AF-Grünland im Juni/Juli 2012 entstanden durch wühlende Wildtiere.

Um signifikante Unterschiede in den mittleren monatlichen N-Nettomineralisationsmengen zwischen den Teilflächen zu bestimmen, wurde die Varianzanalyse bzw. der Kruskal-Wallis-Test verwendet.

Um den Einfluss der anfänglichen extrahierbaren organischen Stickstoffgehalte (eON) auf die mittleren monatlichen $\mathrm{N}$-Nettomineralisationsmengen $\mathrm{zu}$ untersuchen, wurden bivariate Korrelationsanalysen für Reiffenhausen und BERTA durchgeführt.

\subsubsection{Holzige Biomasse}

Um den Anwuchserfolg zu ermitteln, wurden im Frühjahr 2012, also nach einjähriger Standdauer, alle vitalen und toten Bäume der holzigen Anbausysteme KUP-Pappel, KUP-Weide, AF-Weide erfasst.

Zur Abschätzung der holzigen Biomasseproduktion (Holz mit Rinde ohne Laub) der holzigen Anbausysteme nach zweijähriger Standdauer in Reiffenhausen und BERTA, wurde der Brusthöhendurchmesser (BHD, in 1,3 m Höhe) an 240 zufällig ausgewählten Bäumen im Winter 2012/13 bestimmt. Erste Ergebnisse zu möglichen Grünlanderträgen im Agroforstsystem Reiffenhausen im Projekt BEST können unter Ehret et al. (2013) nachgelesen werden.

Um die holzige Biomasse zu bestimmen, wurden pro Anbausystem 15 Probebäume entnommen (Verwijst u. Telenius 1999). Um möglichst das gesamte Spektrum an Brusthöhendurchmessern abzudecken, wurden pro Teilfläche 5 Durchmesserklassen festgelegt, aus denen pro Klasse drei Bäume geerntet wurden. Von diesen Bäumen wurde anschließend im Gelände das erntefrische Gewicht ermittelt. Zur Bestimmung der Trockenbiomasse wurden die Probebäume gehäckselt und bei $105^{\circ} \mathrm{C}$ im Trockenschrank bis zur Gewichtskonstante (absolutes Trockengewicht, atro) getrocknet und anschließend gewogen (Röhle et al. 2006).

Mit Hilfe des Trockengewichtes und den dazugehörigen Brusthöhendurchmessern wurden entsprechende Biomassefunktionen für die verschiedenen Teilflächen berechnet. Wegen der hohen Anpassungsgüte in anderen Studien wurde als Funktionstyp die allometrische Funktion gewählt (Röhle et al. 2006, Röhle 2009, Röhle u. Skibbe 2012, Röhle 2013, Verwijst u. Telenius 1999):

$y=a x^{b}$

wobei $y$ das Trockengewicht $[\mathrm{g}], x$ der BHD $[\mathrm{mm}]$ und $a, b$ die empirisch ermittelten Koeffizienten darstellen. Als Startwert für die Regression wurde der Wert 0,1 festgesetzt. Für die Herleitung der Hektarwerte wurde die Annahme getroffen, dass die gemessene Durchmesserverteilung von 240 
Bäumen pro Teilfläche die jeweilige Grundgesamtheit gut repräsentiert. Die für diese Durchmesserverteilung mit Hilfe der allometrischen Funktion berechneten Biomassewerte wurden, über die Stammzahl pro ha auf Hektarwerte hochgerechnet.

Zur Überprüfung von signifikanten Unterschieden der Brusthöhendurchmesser und der Höhen zwischen den ausgewählten Bäumen der verschiedenen Teilflächen, wurde der Kruskal-Wallis-Test verwendet. Als Post-Hoc-Test wurde der Mann-Whitney-Test verwendet. Dabei wurde darauf geachtet, dass das Signifikanzniveau entsprechend der Anzahl der Paarvergleiche (3 Paarvergleiche) auf 0,017 geändert wurde.

Um den Ernährungszustand der Bestände zu bestimmen, wurden im August 2011/2012 Blattproben entnommen. Nach Rytter u. Ericsson (1993) sollten Blattproben der Korb-Weide (Salix viminalis), im späten Sommer oder Frühherbst genommen werden, damit stabile Blattnährstoffgehalte bestimmt werden können. Auch Röhricht u. Ruscher (2009) kommen zu dem Schluss, dass die Blatternte während der Hauptwachstumsphase der Bäume im August vorgenommen werden sollte. In Reiffenhausen wurden auf der Teilfläche KUP-Pappel die Reihen 1, 7, 12, 17, 26 und auf der Teilfläche KUP-Weide die Reihen 1, 7, 12, 17, 22, 44 beprobt (Abb. 10). Auf der Teilfläche AF-Weide in Reiffenhausen und BERTA wurden Proben von allen Reihen genommen. Von der KUP-Weide und KUP-Pappel in BERTA wurden die Reihen 1, 12, 17, 22, 34 beprobt (Abb. 10).
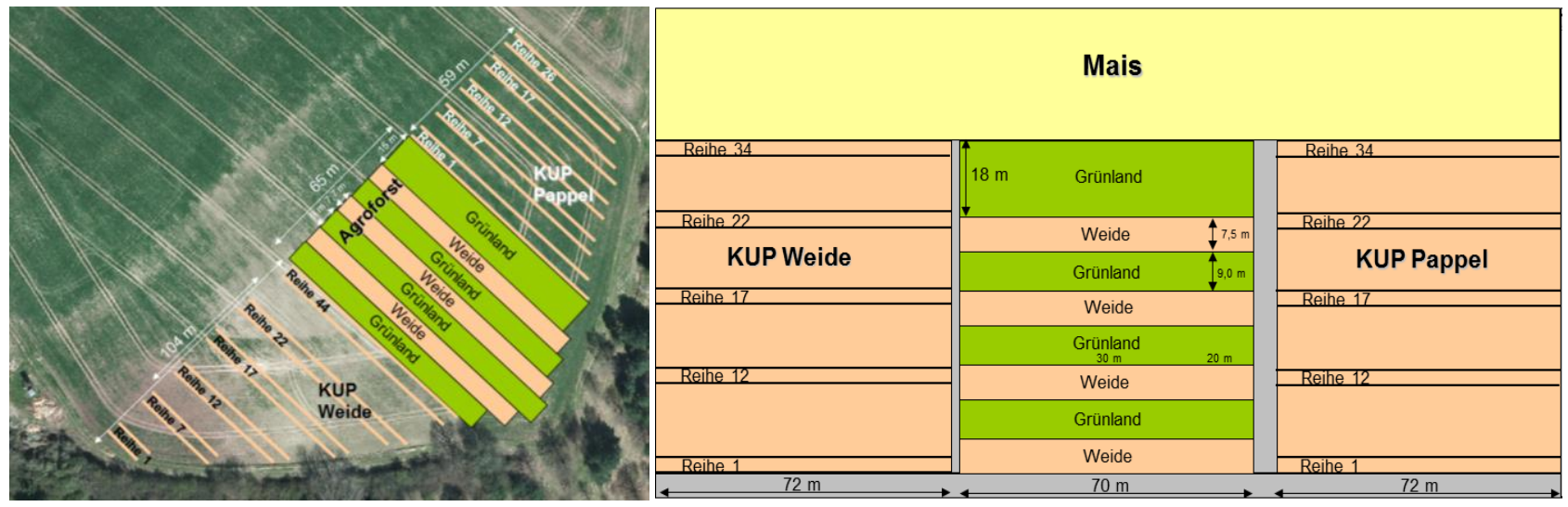

Abb. 10 Überblick zu den beprobten Doppelreihen in Reiffenhausen (links) und BERTA (rechts) von denen Blätter für die Bestimmung des Ernährungszustandes der Bestände gewonnen wurden. Datengrundlage: Reiffenhausen: Digitales Orthophoto 2010, Quelle: Auszug aus den Geobasisdaten der Niedersächsischen Vermessungs- und Katasterverwaltung, (c) 2011 Landesamt für Geoinformation und Landentwicklung Niedersachsen (LGLN)

Bei der Auswahl der Reihen wurde darauf geachtet, dass die gesamte Teilfläche, d.h. auch die Randbereiche erfasst wurden. Es wurden stets gesunde Blätter aus dem oberen Drittel der Bäume beprobt. Die Blätter wurden bei $60{ }^{\circ} \mathrm{C}$ und die komplett gehäckselten Probebäume bei $105{ }^{\circ} \mathrm{C}$ bis zur Gewichtskonstante getrocknet und anschließend fein gemahlen (Retsch ZM 1000, Retsch, Haan, Germany). Der Gehalt an Phosphor [mg (P) $\mathrm{g}^{-1}$ ], Kalium [mg (K) $\mathrm{g}^{-1}$ ], Calcium [mg (Ca) $\mathrm{g}^{-1}$ ], und Magnesium [mg (Mg) g ${ }^{-1}$ ], wurde nach $\mathrm{HNO}_{3}$-Druckaufschluss mittels ICP-AES (Inductively Coupled 
Plasma-Atomic Emission Spectrometer, Fa. Thermo Fisher) bestimmt. Die Gehalte an Kohlenstoff [mg (C) $\mathrm{g}^{-1}$ ] und Stickstoff [mg (N) $\mathrm{g}^{-1}$ ] wurden mittels oxidativer Verbrennung am C-N-Analysator (CHN-ORapide, VarioEL, Fa. Elementar) bestimmt. Aus den Einzelwerten wurde dann der Mittelwert für die gesamte Teilfläche berechnet.

Der Nährstoffentzug durch die holzige Biomasse wurde aus der Kombination des Zuwachses und den mittleren Nährstoffgehalten der Probebäume wie folgt berechnet:

$N=Z * G$

wobei $\mathrm{N}$ den Nährstoffentzug $\left[\mathrm{kg} \mathrm{ha}^{-1}\right], \mathrm{Z}$ den Zuwachs $\left[\mathrm{kg}_{\text {atro }} \mathrm{ha}^{-1} \mathrm{a}^{-1}\right]$ und $\mathrm{G}$ den Nährstoffgehalt $\left[\mathrm{g} \mathrm{kg}^{-1}\right]$ darstellt (Boelcke u. Kahle 2008).

\subsubsection{Laubproduktion und Umsatz}

Von Oktober 2011 bis Dezember 2011 und von Oktober 2012 bis Dezember 2012 wurde das eingetragene Laub von sechs Probequadraten (Fläche: $1 \mathrm{~m}^{2}, 1 \mathrm{~m} \times 1 \mathrm{~m}$ ) pro holzigen Anbausystem gesammelt und die Höhe [m] der Bäume gemessen.

Das Laub wurde bei Bedarf (anhaftender Boden) vorsichtig gewaschen und anschließend bei $60^{\circ} \mathrm{C}$ getrocknet, danach gewogen und fein gemahlen (Retsch ZM 1000, Fa. Retsch). Das Trockengewicht wurde anschließend auf einen Hektar bezogen $\left[\mathrm{kg} \mathrm{ha}^{-1}\right.$ ]. Die Gehalte an Kalium [mg (K) g ${ }^{-1}$ ], Calcium [mg (Ca) $\mathrm{g}^{-1}$ ] und Magnesium [mg (Mg) g ${ }^{-1}$ ] wurden nach $\mathrm{HNO}_{3}$-Druckaufschluss mittels ICP-AES bestimmt. Die Gehalte an Kohlenstoff $\left[\mathrm{mg}(\mathrm{C}) \mathrm{g}^{-1}\right]$ und Stickstoff $\left[\mathrm{mg}(\mathrm{N}) \mathrm{g}^{-1}\right.$ ] wurden mittels oxidativer Verbrennung am C-N-Analysator bestimmt. Durch Multiplikation der Nährstoffgehalte [g $\left.\mathrm{kg}^{-1}\right]$ mit dem Gewicht des eingetragenen Laubes $\left[\mathrm{kg} \mathrm{ha}^{-1}\right]$ wurde die eingetragene Nährstoffmenge [g ha ${ }^{-1}$ bzw. kg ha ${ }^{-1}$ ] berechnet.

Zum Nachweis signifikant unterschiedlicher eingetragener Laubmengen und Baumhöhen zwischen den Teilflächen wurde ein Kruskal-Wallis-Test berechnet. Als Post-Hoc-Test diente der MannWhitney-Test. Dabei wurde darauf geachtet, dass das Signifikanzniveau entsprechend der Anzahl der Paarvergleiche (3 Paarvergleiche) auf 0,017 geändert wurde.

Der Laubumsatz wurde mittels eingebrachter Litterbags bestimmt. Dazu wurden ca. $15 \mathrm{~cm} \times 15 \mathrm{~cm}$ große Beutel aus Polyester genäht, in denen 2,50 g Laub eingewogen wurden. Die ausgebrachten Litterbags wiesen eine Maschenweite von $1 \mathrm{~mm}$ auf, sodass gewisse Bodenorganismen (z.B. Regenwürmer) von vornherein ausgeschlossen wurden. In der Literatur wurde darauf hingewiesen, dass bei größeren Maschenweiten eine Unterscheidung zwischen Laub und Boden (Streueinarbeitung und Wurmlosung) nur schwer möglich wäre, weswegen in dieser Arbeit engmaschige Litterbags eingesetzt wurden (Prescott 2005, Šlapokas u. Granhall 1991). Das Weiden- 
und Pappellaub für die Litterbags wurde von einer angrenzenden Kurzumtriebsplantage in der "Thüringer Ackerebene" gewonnen, da unsere Bestände noch zu wenig Material lieferten. Das Pappellaub stammte vom Klon "Japan" (4 Jahre alt) und das Weidenlaub vom Klon "Tora" (8 Jahre alt). Das gesammelte Laub wurde mindestens 1 Woche an der Luft getrocknet und dabei mehrmals gewendet. Eine Teilmenge des Laubes wurde zur anfänglichen Nährstoffanalyse in vierfacher Wiederholung entnommen und bei $60{ }^{\circ} \mathrm{C}$ getrocknet und anschließend fein gemahlen (Retsch ZM 1000, Fa. Retsch).

Im November 2011 wurden in den Beprobungsbereichen der Teilflächen (KUP-Pappel, KUP-Weide, AF-Weide) jeweils 16 Litterbags ausgebracht. Demzufolge wurden auf beiden Standorten je 48 Litterbags ausgebracht. Diese wurden mit Stäben befestigt, wobei darauf geachtet wurde, dass Bodenkontakt bestand. Im vierteljährigen Rhythmus also im Februar, März, August und November 2012 wurden die Litterbags wieder eingeholt und ggf. gereinigt (Entfernung von Bodenmaterial). Das entnommene Laub wurde dann mindestens eine Woche an der Luft getrocknet und gewogen. Anschließend wurde es bei $60{ }^{\circ} \mathrm{C}$ getrocknet und fein gemahlen (Retsch ZM 1000, Fa. Retsch). Die Nährstoffgehalte (anfängliche und nach der Einholung der Litterbags) wurden nach $\mathrm{HNO}_{3}-$ Druckaufschluss mittels ICP-AES und oxidativer Verbrennung am C-N-Analysator bestimmt.

Der Gewichtsverlust des Laubes in den Litterbags wurde nach der Formel (4) berechnet (Guo u. Sims 1999, 2001).

$L=\frac{W o-W t}{W o} * 100$

wobei $L$ [\%] der Gewichtsverlust des Laubes, Wo das Anfangsgewicht [g] bei Ausbringung der Litterbags und Wt das Endgewicht [g] nach Einholung der Litterbags darstellt.

Die Bestimmung der Nährstofffreisetzung aus dem Laub in den Litterbags wurde nach der Formel (5) berechnet (Guo u. Sims 1999, 2001).

$R=\frac{W o C o-W t C t}{W o C o} * 100$

wobei R [\%] die Nährstofffreisetzung des Laubes, Wo das Anfangsgewicht [g] und Co der Nährstoffgehalt des Laubes [ $\mathrm{mg} \mathrm{g}^{-1}$ ] bei Ausbringung der Litterbags und Wt das Endgewicht [g] und Co der Nährstoffgehalt des Laubes [ $\mathrm{mg} \mathrm{g}^{-1}$ ] nach Einholung der Litterbags darstellt.

Signifikante Unterschiede zwischen den Gewichtsverlusten und der Freisetzung an Nährstoffen der Litterbags zwischen den Teilflächen (KUP-Pappel, KUP-Weide, AF-Weide) wurden mit Hilfe der Varianzanalyse mit nachgeschalteten Post-Hoc Test (Tamhane-T2/Tukey) ermittelt. 
Um die Zersetzung des Laubes mit den Daten anderer Studien vergleichen zu können, wurde die tägliche Zersetzungsrate bestimmt (Guo u. Sims 1999). Diese wurde mit Hilfe der linearen Regressionsfunktion (6) ermittelt, da diese eine höhere Anpassungsgüte (Jacob et al. 2010) im Vergleich zur häufig verwendeten exponentiellen Funktion lieferte (Bärlocher 2005, Guo u. Sims 1999, 2001, Olson 1963, Polyakova u. Billor 2007).

$\mathrm{G}=a-k t$

wobei $G[\mathrm{~g}$ ] das Gewicht zu den verschiedenen Entnahmeterminen, $a$ Regressionskonstante, $k$ tägliche Zersetzungsrate und $t$ Zeit (d) darstellt.

Zum Nachweis signifikant unterschiedlicher Zersetzungsraten zwischen den verschiedenen Teilflächen (KUP-Pappel, KUP-Weide, AF-Weide) wurde eine Varianzanalyse berechnet. Als Post-HocTest wurde unter Berücksichtigung der Varianzhomogenität der Tukey-Test angeschlossen.

Signifikante Unterschiede zwischen den Nährstoffgehalten des eingetragenen Laubes und des ausgebrachten Laubes in den Litterbags wurden mit Hilfe der Varianzanalyse, mit anschließendem Post-Hoc Test (Tamhane-T2, Tukey) in Abhängigkeit von Varianzhomogenität bzw. Varianzheterogenität ermittelt.

\subsubsection{Boden- und Sickerwasser}

Im August 2011 wurden 5 Saugkerzen pro Teilfläche in einer Tiefe von $60 \mathrm{~cm}$ mit einem Winkel von $45^{\circ}$ zur Bodenoberfläche installiert. Die Saugkerzenanlage besteht aus einem porösen, keramischen Körper (P-80 cup, $5 \times 2 \mathrm{~cm}$, Fa. CeramTec AG) mit einem fest installierten PVC-Schaft, Verbindungsschläuchen zur Erdoberfläche und einem Auffanggefäß, das sich in einer Gefrierkiste befand. Auf der Teilfläche Acker wurden die Saugkerzen parallel zur Bodenoberfläche eingebaut, um eine problemlose Bewirtschaftung zu gewährleisten.

Zur Gewinnung des Bodenwassers wurde ein Unterdruck von 0,7 bar angelegt (DVWK - Deutscher Verband für Wasserwirtschaft und Kulturbau 1990). Die Beprobung der Bodenlösung erfolgte diskontinuierlich in mindestens zweiwöchigem Abstand (Lord u. Shepherd 1993). Die Saugkerzen wurden sowohl in der Doppelreihe als auch in den Fahrgassen diagonal installiert. Das Bodenwasser wurde kühl (maximal $4{ }^{\circ} \mathrm{C}$ ) und dunkel bis zur Analyse gelagert.

Die Analyse des gelösten Stickstoffs im Bodenwasser [mg (N) $\mathrm{L}^{-1}$ ] des Gesamtstickstoffs (TDN), des Ammoniums $\left(\mathrm{DNH}_{4}\right)$, des Nitrats $\left(\mathrm{DNO}_{3}\right)$ und des organischen Stickstoffs (DON $\left.=\mathrm{TDN}-\mathrm{DNH}_{4}-\mathrm{DNO}_{3}\right)$ erfolgte mittels Continuous-Flow-Injection-Colorimetry (Cenco/Skalar Instruments). Trotz sorgefältiger Beprobung lieferten nicht alle Saugkerzen konstant Bodenwasser, sodass zu einigen Terminen keine Proben von den verschiedenen Teilflächen gewonnen werden konnten. 
Um die Bodenwasserkonzentrationen zwischen den verschiedenen Teilflächen miteinander zu vergleichen, wurde aus den über ein Jahr gemessenen Konzentrationen je ein Mittelwert pro Teilfläche gebildet.

Um den Nitrataustrag mit dem Sickerwasser innerhalb eines Jahres abzuschätzen, wurden die fehlenden Nitratgehalte $\left(\mathrm{DNO}_{3}\right)$ im Bodenwasser durch die vorhandenen Vormonatswerte ersetzt, bzw. zwischen zwei Zeiträumen interpoliert und mit der Sickerwassermenge multipliziert. Die Sickerwassermengen für den Zeitraum Dezember 2011 bis November 2012 in Reiffenhausen und Januar 2012 bis Dezember 2012 in BERTA wurden im Teilprojekt "Wasserhaushalt" von BEST (Bearbeitung Falk Richter, Abteilung Ökopedologie der gemäßigten Zonen, Georg-August Universität Göttingen) für die Teilfläche KUP-Pappel modelliert. Diese Sickerwassermengen wurden auf die anderen holzigen Anbausysteme übertragen, sodass an dieser Stelle nur eine grobe Abschätzung für den Nitrataustrag für KUP-Weide bzw. AF-Weide erfolgen kann.

\subsubsection{Deposition}

Ab November 2011 bis Oktober 2012 wurden in mindestens zweiwöchigem Turnus Depositionsproben mit Schneeeimern und Bulk-Sammlern gewonnen und anschließend ausgewogen $[\mathrm{kg}]$. Bis zur Analyse wurden die Proben dunkel und kühl (maximal $4{ }^{\circ} \mathrm{C}$ ) gelagert. Die Schneeeimer hatten eine Auffangfläche von $500 \mathrm{~cm}^{2}$ und wurden in 1,2 $\mathrm{m}$ Höhe installiert. Die Bulk-Sammler, welche ebenfalls in 1,2 m Höhe aufgestellt wurden, hatten eine Grundfläche von $86,54 \mathrm{~cm}^{2}$. In Reiffenhausen und BERTA wurden jeweils 4 Schneeeimer und 4 Bulk-Sammler in unmittelbarer Nähe zu den Kurzumtriebsplantagen installiert. Die Schneeeimer befanden sich direkt auf den Versuchsflächen. Durch die Bauweise der Bulk-Sammler (Trichter und Insektengitter) konnten Verdunstungsverluste und Verunreinigungen der Proben minimiert werden (Klinck et al. 2013). Die Flächengrößen der Bulk-Sammler und Schneeeimer wurden anschließend auf $1 \mathrm{~m}^{2}$ bezogen. Aus den vier Wiederholungen des gewonnenen Niederschlags wurde stets der Mittelwert gebildet, welcher anschließend mit den Konzentrationen an gelöstem Ammonium $\left(\mathrm{DNH}_{4}\right)$, Nitrat $\left(\mathrm{DNO}_{3}\right)$ und Gesamtstickstoff (TDN) [mg (N) $\left.\mathrm{L}^{-1}\right]$ multipliziert wurde. Weiterhin wurde dieser Wert $\left[\mathrm{mg}(\mathrm{N}) \mathrm{m}^{-2}\right]$ dann auf einen Hektar bezogen und auf einen Zeitraum von einem Jahr aufsummiert (Jahresdeposition). Die Bestimmung der Stickstoffkonzentrationen erfolgte mittels Continuous-FlowAnalyse (Cenco/Skalar Instruments). Die trockene Deposition der aufwachsenden Bestände wurde vernachlässigt, da Untersuchungen zum Depositionsgeschehen in Ökosystemen mit niedriger Bodenvegetation dies rechtfertigen (Klinck et al. 2013). 


\subsubsection{N-Bilanz und N-Nutzungseffzienz}

Zur Erstellung der N-Bilanz wurden verschiedene Ein- und Austragsgrößen herangezogen. Im Detail wurden zur Bilanzierung des N-Eintrages die N-Nettomineralisation (Kapitel 2.2.2) und Deposition (Kap. 2.2.6) berücksichtigt. Der N-Austrag wurde aus der Lachgasemission und dem Sickerwasseraustrag berechnet (Kapitel 2.2.5). Da die jährlichen Lachgasemissionen in einem weiteren Teilprojekt von BEST ("Klimaschutzleistung", Bearbeiterin Katja Walter, Agrarklimaschutz, vTi Braunschweig) lediglich für das holzige Anbausystem KUP-Pappel in Reiffenhausen und BERTA von 2011-2013 ermittelt wurden, können die Emissionen für die anderen holzigen Anbausysteme nur abgeschätzt werden. Dazu wurden die Austragswerte von der KUP-Pappel auf die anderen holzigen Anbausysteme übertragen.

Nach Asplund et al. (2014), Moll et al. (1982) und Weih et al. (2011) untergliedert sich die Stickstoffnutzungseffizienz (Nitrogen Use Efficiency) in die Stickstoffzuwachseffizienz (Nitrogen Utilization Efficiency) und Stickstoffaufnahmeeffizienz (Nitrogen Uptake Efficiency). Statt die NZuwachseffizienz wird von verschiedenen Autoren wie Adegbidi et al. (2001), Berthelot et al. (2000), Calfapietra et al. (2007), Jørgensen u. Schelde (2001), Lodhiyal et al. (1995) und Lodhiyal u. Lodhiyal (1997a) auch N-Nutzungseffizienz als synonymer Begriff verwendet. In dieser Arbeit werden die Begriffe nach Moll (1982) gebraucht. Unter N-Zuwachseffizienz versteht man das Verhältnis zwischen dem Zuwachs an holziger Biomasse (Kapitel 2.2.3) und dem N-Entzug mit der Ernte der holzigen Biomasse (Kapitel 2.2.3). Die N-Aufnahmeeffizienz ergibt sich aus dem Verhältnis von NEntzug durch Ernte der holzigen Biomasse zum bereitgestellten Stickstoff im Boden (in diesem Fall die N-Nettomineralisation in der Vegetationsperiode, Kapitel 2.2.2) und der atmosphärischen Deposition (Kapitel 2.2.6).

\section{Ergebnisse}

Die in diesem Kapitel vorgestellten Ergebnisse wurden teilweise in Tagungsbänden, einer Fachzeitschrift und in einem Buchbeitrag veröffentlicht. Ausführliche Informationen zu den standörtlichen Gegebenheiten der Versuchsflächen können den Beiträgen Hartmann et al. (2014) und Hartmann u. Lamersdorf (2014a) entnommen werden. Ertragsdaten zur holzigen Biomasse finden sich in Hartmann et al. (2013), Hartmann et al. (2014) und Hartmann u. Lamersdorf (2014b). Die Stickstoffvorräte und -Flüsse (u.a. N-Nettomineralisation, Deposition) für die Versuchsfläche Reiffenhausen wurden im Buchbeitrag Hartmann u. Lamersdorf (2014a) dargestellt. 


\subsection{Standortcharakteristik}

\subsubsection{Klima}

Für ein ertragreiches Wachstum schnellwachsender Gehölze sollte die Jahresdurchschnittstemperatur mindestens $6,5^{\circ} \mathrm{C}$ betragen (Aust 2012, Petzold et al. 2010a). Diese Anforderung ist in beiden Regionen mit 9,2 ${ }^{\circ} \mathrm{C}$ im Ldk. Gö. bzw. 9, $4{ }^{\circ} \mathrm{C}$ in der "Thüringer Ackerebene" erfüllt.

Neben einem ausreichenden Wärmeangebot ist auch die Wasserversorgung der Pflanzen bedeutsam. Jahresniederschläge von $500 \mathrm{~mm}$ bzw. $300 \mathrm{~mm}$ während der Vegetationsperiode (MaiSeptember) gelten als ausreichend für das Erreichen hoher Zuwächse von Pappeln und Weiden (Petzold et al. 2006, Petzold et al. 2010a). Im langjährigen Jahresmittel (1991-2010) liegen beide Regionen über dem Grenzwert von 500 mm (Ldk. Gö. 642 mm, "Thüringer Ackerebene" 549 mm) und eignen sich demnach gut für einen langfristig ertragreichen Anbau von KUP. Die Niederschlagsmengen während der Vegetationsperiode (Mai-September, Periode 1991-2010) ergeben ein differenzierteres Bild: im Gegensatz zur Versuchsfläche Reiffenhausen im Ldk. Gö. (312 $\mathrm{mm}$ ) wird in der "Thüringer Ackerebene" $(289 \mathrm{~mm}$ ) die empfohlene Mindestniederschlagsmenge von $300 \mathrm{~mm}$ nicht erreicht (Tabelle 1).

Im Vergleich zum Landkreis Göttingen fällt in der "Thüringer Ackerebene" im langjährigen Mittel nicht genügend Niederschlag in der Vegetationsperiode für ein ertragreiches Wachstum der Pappeln und Weiden.

\subsubsection{Leitprofile}

Das erste Profil (Reiffenhausen Referenz (RR), Abb. 7, links) liegt in der lössüberprägten Solling-Folge des Mittleren Buntsandstein und ist im Oberboden durch eine typisch sandig-lehmige Bodenart gekennzeichnet. In den Laboranalysen konnten Carbonatgehalte von ca. 0,3-1,1\% nachgewiesen werden (Tabelle 4). Es wird vermutet, dass es sich um Dolomit handelt, der deutlich langsamer auf die Zugabe von Salzsäure reagiert. Im Allgemeinen kann Buntsandstein auch geringe Mengen von Carbonat als Bindemittel enthalten, insbesondere in den paläogeographisch weiter östlich gelegenen Gebieten (Ziegler 1990). Der Bodentyp wurde als schwach pseudovergleyte Braunerde angesprochen.

Das zweite Profil (Reiffenhausen Weide (RW), Abb. 7, links) liegt ca. $200 \mathrm{~m}$ hangaufwärts in der KUPWeide und hat sich auf Oberem Buntsandstein (Röt) entwickelt, der ebenfalls Carbonate enthält (Tabelle 4). Das Profil zeigt im Oberboden eine Reaktion mit Salzsäure, sowohl im Skelett, als auch im Feinboden. Bei den Bauarbeiten zum Heidkopftunnel der Autobahn A38 konnte bei Profilbohrungen ebenfalls der dolomitische Obere Buntsandstein (soD) nachgewiesen werden (Arp et al. 2011a, DEGES 2009, Wedell 2013a, 2013b). Im Vergleich zum Profil der Sollingfolge sind die Bodenarten hier 
im oberen Bereich deutlich schluffiger, toniger und in den tieferen Bodenhorizonten lehmiger. Das Profil zeigt bis ca. $35 \mathrm{~cm}$ hochreichende hydromorphe Bedingungen und wurde daher als Pseudogley angesprochen. Eine deutliche Unterscheidung zwischen Sd- und Sw-Horizont war allerdings nicht möglich.

Das dritte Leitprofil befindet sich in der "Thüringer Ackerebene" und wird im Folgenden BERTA (B) genannt (Abb. 7, rechts). Es liegt auf einem relativ schmalen Rücken aus flachgründigen Gips- bzw. Mergelsteinen des Mittleren Keupers (Neumann 1883), die von Lößfließerden überlagert wurden. Das stark schluffige, skelettfreie Solum beschränkt sich auf die obersten $50 \mathrm{~cm}$, darunter steigt der Skelettgehalt auf über $50 \%$ an. Als Bodentyp wurde eine Rendzina-Braunerde angesprochen. Die Carbonatgehalte liegen im Ap-Horizont bei 4,6\%.

Der pH-Wert lässt Aussagen über die relative Verfügbarkeit von Nährelementen zu und steuert wichtige bodenbildende Prozesse. Die geringsten $\mathrm{pH}$-Werte aller untersuchten Proben liegen mit 5,8 im schwach sauren Bereich und wurden im Oberboden $(0-10 \mathrm{~cm})$ für das Leitprofil RR nachgewiesen (Tabelle 4). Im Leitprofil RW liegen neutrale Bodenreaktionen vor. Das Leitprofil B weist schwach alkalische pH-Werte auf (Blume et al. 2011). Sämtliche Profile befinden sich annähernd im optimalen Bereich (pH-Wert: 6,0-7,5) für Pappeln und Weiden (Röhricht u. Ruscher 2009) und lassen demzufolge gute Bedingungen für das Pflanzenwachstum erwarten. Grundsätzlich steigen die pHWerte mit zunehmender Bodentiefe an. Mit pH-Werten größer als 6,2 liegen die Profile RW und B im Carbonatpufferbereich. In RR wird aufgrund der geringen und ungleichmäßigen Verteilung der Carbonate auch der Silikatpuffer $(6,2>\mathrm{pH}$-Wert $>5,0)$ wirksam (Arbeitskreis Standortskartierung 2003).

Alle Leitprofile weisen mit zunehmender Tiefe eine Abnahme der $\mathrm{C}_{\text {org }}$-Gehalte auf (Tabelle 4). Die höchsten Konzentrationen werden für das Leitprofil B verzeichnet. In den Ap-Horizonten reichen die

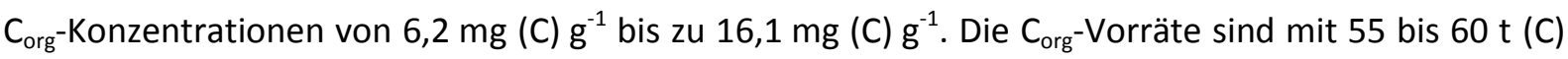
$\mathrm{ha}^{-1}$ (für B bezogen auf die Bodentiefe 0-30 cm und für RR, RW auf die Bodentiefe 0-90 cm) ähnlich. Nach den Bewertungsrahmen des Arbeitskreises Standortskartierung (2003) weisen die Standorte geringe Vorräte an organischem Kohlenstoff auf.

An allen untersuchten Standorten liegt Kohlenstoff auch in anorganischer Form, als Carbonat, vor. Die höchsten Gehalte im Oberboden $(0-30 \mathrm{~cm})$ wurden mit ca. 4,6\% für das Leitprofil B nachgewiesen (Tabelle 4), was mit den anstehenden Mergelgesteinsablagerungen erklärt werden kann. 
Tabelle 4 Mittlere (Standardabweichung) bodenchemische Kennwerte $(n=3)$ : pH-Wert; Gesamtgehalt und Vorrat an $\mathrm{C}_{\text {org }}, \mathrm{N}_{\mathrm{t}}$, Gehalt an $\mathrm{CaMg}\left(\mathrm{CO}_{3}\right)_{2}$ und $\mathrm{CaCO}_{3}$ sowie $\mathrm{C}_{\text {org }} / \mathrm{N}_{\mathrm{t}}-$ Verhältnis der Leitprofile (LP) Reiffenhausen Referenz (RR), Reiffenhausen Weide (RW) und BERTA (B)

\begin{tabular}{ccccccccc}
\hline LP & Tiefe & $\mathrm{pH}$ & $\mathrm{C}_{\text {org }}$ & $\mathrm{C}_{\text {org }}$ & $\mathrm{CaMg}\left(\mathrm{CO}_{3}\right)_{2}, \mathrm{CaCO}_{3} *$ & $\mathrm{~N}_{\mathrm{t}}$ & $\mathrm{N}_{\mathrm{t}}$ & $\mathrm{C}_{\text {org }} / \mathrm{N}_{\mathrm{t}}$ \\
\cline { 2 - 8 } & {$[\mathrm{cm}]$} & {$\left[\mathrm{H}_{2} \mathrm{O}\right]$} & {$\left[\mathrm{mg} \mathrm{g}^{-1}\right]$} & {$\left[\mathrm{t} \mathrm{ha}^{-1}\right]$} & {$[\%]$} & {$\left[\mathrm{mg} \mathrm{g}^{-1}\right]$} & {$\left[\mathrm{t} \mathrm{ha}^{-1}\right]$} & \\
\hline RR & $0-10$ & $5,8(0,2)$ & $10,9(0,3)$ & $13,8(0,4)$ & $1,2(0,6)$ & $1,2(0,0)$ & $1,5(0,1)$ & $9,0(0,5)$ \\
& $10-30$ & $6,0(0,2)$ & $8,6(1,5)$ & $26,9(4,7)$ & $1,0(0,4)$ & $1,0(0,2)$ & $3,2(0,6)$ & $8,4(1,0)$ \\
& $30-60$ & $6,5(0,1)$ & $2,9(0,7)$ & $12,9(3,3)$ & $0,4(0,2)$ & $0,4(0,1)$ & $1,8(0,4)$ & $7,1(0,3)$ \\
& $60-90$ & $6,7(0,1)$ & $1,2(0,1)$ & $5,3(0,6)$ & $0,4(0,1)$ & $0,2(0,1)$ & $0,8(0,2)$ & $6,8(2,4)$ \\
& & & & & & & & \\
RW & $0-10$ & $7,1(0,3)$ & $13,0(0,3)$ & $17,8(0,5)$ & $1,4(0,4)$ & $1,5(0,1)$ & $2,0(0,1)$ & $8,7(0,6)$ \\
& $10-30$ & $7,1(0,2)$ & $6,2(1,2)$ & $18,5(3,5)$ & $0,2(0,1)$ & $0,7(0,1)$ & $2,2(0,2)$ & $8,4(1,0)$ \\
& $30-60$ & $7,3(0,1)$ & $3,3(0,2)$ & $14,6(0,8)$ & $0,6(0,1)$ & $0,6(0,0)$ & $2,8(0,1)$ & $5,2(0,4)$ \\
& $60-90$ & $7,9(0,0)$ & $1,3(0,2)$ & $4,0(0,7)$ & $30,1(5,3)$ & $0,4(0,1)$ & $1,1(0,2)$ & $3,6(0,1)$ \\
& & & & & & & \\
B & $0-10$ & $8,0(0,1)$ & $16,1(0,8)$ & $17,4(0,8)$ & $4,7(1,1)$ & $1,6(0,1)$ & $1,7(0,1)$ & $10,3(0,4)$ \\
& $10-30$ & $8,2(0,0)$ & $15,5(0,6)$ & $42,0(1,7)$ & $4,6(2,3)$ & $1,4(0,1)$ & $3,8(0,1)$ & $10,9(0,1)$ \\
\hline
\end{tabular}

Während $\mathrm{C}_{\text {org }}$ im Laufe mikrobiellen Abbaus zum größten Teil als Kohlenstoffdioxid freigesetzt wird, wird Stickstoff zu mehr als $95 \%$ in organischer Bindung stabilisiert (Blume et al. 2010). Der Gesamtstickstoffgehalt $\left(\mathrm{N}_{\mathrm{t}}\right)$ beträgt in den Ap-Horizonten von Mineralböden meist 0,7 bis $2 \mathrm{mg}$ (N) $\mathrm{g}^{-1}$, sodass $\mathrm{N}_{\mathrm{t}}$-Vorräte von 3 bis $9 \mathrm{t}(\mathrm{N}) \mathrm{ha}^{-1}$ möglich sind (Blume et al. 2010). In den Ap-Horizonten der Leitprofile reichen die $\mathrm{N}_{\mathrm{t}}$-Gehalte von $0,7 \mathrm{mg}(\mathrm{N}) \mathrm{g}^{-1}$ bis $1,6 \mathrm{mg}(\mathrm{N}) \mathrm{g}^{-1}$ (Tabelle 4). Die Stickstoffvorräte, bezogen auf die Bodentiefe 0-90 cm für die Leitprofile RR, RW und 0-30 cm für das Leitprofil B, können nach Tabelle 3 der "mittleren" Klasse zugeordnet werden.

Die Qualität der organischen Substanz ist umso besser, je stickstoffreicher diese ist, d.h. je enger ihr Verhältnis an organischem Kohlenstoff zum Gesamtstickstoff $\left(\mathrm{C}_{\mathrm{org}} / \mathrm{N}_{\mathrm{t}}\right)$ ist. Die $\mathrm{C}_{\text {org }} / \mathrm{N}_{\mathrm{t}}-$ Verhältnisse im Ap-Horizont der untersuchten Standorte liegen zwischen 8,4 und 10,9 (Tabelle 4) und sind demzufolge sehr eng (Arbeitskreis Standortskartierung 2003). Dies deutet auf günstige Umsatzbedingungen hin, welche den Abbau der organischen Substanz begünstigen.

Zur Beurteilung der Bodenfruchtbarkeit wurde die potenzielle Kationenaustauschkapazität (KAK pot) herangezogen. Die $\mathrm{KAK}_{\text {pot }}$ aller untersuchten Leitprofile variiert zwischen $60-230 \mathrm{mmol}_{\mathrm{c}} \mathrm{kg}^{-1}$ und nimmt in der Regel mit zunehmender Tiefe ab (Tabelle 5). In den Ap-Horizonten ist die KAK ${ }_{\text {pot }}$ i.d.R. am größten, was durch einen erhöhten Humusgehalt bedingt wird. Das höchste Bindungsvermögen für Nährstoffe im Ap-Horizont konnte im Leitprofil B mit ca. $224 \mathrm{mmol}_{\mathrm{c}} \mathrm{kg}^{-1}$ beobachtet werden. Damit ist die $\mathrm{KAK}_{\text {pot }}$ der untersuchten Tiefenstufen für alle Profile als "mittel" einzustufen (Tabelle 3, Tabelle 5). In RW wirken sich die höheren Tongehalte von ca. $40 \%$ positiv auf die $K A K_{\text {pot }}$ aus, im Vergleich zu RR mit Tongehalten von ca. $15 \%$. 
Tabelle 5 Mittlere (Standardabweichung) bodenchemische Kennwerte $(n=3)$ : Potenzielle Kationenaustauschkapazität $\left(\mathrm{KAK}_{\mathrm{pot}}\right)$, Basensättigung (BS) und Kationensättigung sowie Vorrat an Natrium (Na), Kalium (K), Calcium (Ca), Magnesium (Mg) und Gesamtphosphor $\left(P_{t}\right)$ der Leitprofile (LP) Reiffenhausen Referenz (RR), Reiffenhausen Weide (RW) und BERTA (B)

\begin{tabular}{|c|c|c|c|c|c|c|c|c|c|c|c|c|}
\hline \multirow[t]{2}{*}{ LP } & \multirow{2}{*}{$\begin{array}{l}\text { Tiefe } \\
{[\mathrm{cm}]}\end{array}$} & \multirow{2}{*}{$\begin{array}{l}\mathrm{KAK}_{\text {pot }} \\
{\left[\mathrm{mmol}_{\mathrm{c}}\right.} \\
\left.\mathrm{kg}^{-1}\right]\end{array}$} & \multirow{2}{*}{$\begin{array}{l}\text { BS } \\
{[\%]}\end{array}$} & \multicolumn{4}{|c|}{ Sättigung \% von KAK ${ }_{\text {pot }}$} & \multirow{2}{*}{$\begin{array}{l}\mathrm{Na} \\
\mathrm{t} \\
\left.\mathrm{ha}^{-1}\right]\end{array}$} & \multirow{2}{*}{$\begin{array}{l}\mathrm{K} \\
\mathrm{t} \\
\left.\mathrm{ha}^{-1}\right]\end{array}$} & \multirow{2}{*}{$\begin{array}{l}\mathrm{Ca} \\
\mathrm{t} \\
\left.\mathrm{ha}^{-1}\right]\end{array}$} & \multirow{2}{*}{$\begin{array}{l}\mathrm{Mg} \\
\mathrm{t} \\
\left.\mathrm{ha}^{-1}\right]\end{array}$} & \multirow{2}{*}{$\begin{array}{l}P_{t} \\
{[\mathrm{t}} \\
\left.\mathrm{ha}^{-1}\right]\end{array}$} \\
\hline & & & & $\mathrm{Na}$ & $\mathrm{K}$ & $\mathrm{Ca}$ & $\mathrm{Mg}$ & & & & & \\
\hline \multirow{8}{*}{$\mathrm{RR}$} & $0-$ & 105,5 & 77,1 & 0,3 & 7,0 & 51,3 & 18,4 & 0,0 & 0,4 & 2,8 & 0,6 & 0,8 \\
\hline & 10 & $(0,8)$ & $(19,9)$ & $(0,3)$ & $(2,9)$ & $(3,0)$ & $(19,6)$ & $(0,0)$ & $(0,2)$ & $(0,2)$ & $(0,6)$ & $(0,0)$ \\
\hline & $10-$ & 99,6 & 68,7 & 0,3 & 7,4 & 53,4 & 7,6 & 0,0 & 0,9 & 6,7 & 0,6 & 1,8 \\
\hline & 30 & $(1,2)$ & $(1,2)$ & $(0,4)$ & $(0,2)$ & $(0,8)$ & $(0,3)$ & $(0,0)$ & $(0,0)$ & $(0,1)$ & $(0,0)$ & $(0,0)$ \\
\hline & $30-$ & 86,0 & 79,5 & 0,0 & 7,2 & 64,4 & 7,9 & 0,0 & 1,1 & 9,7 & 0,7 & 1,3 \\
\hline & 60 & $(9,6)$ & $(16,0)$ & $(0,0)$ & $(0,9)$ & $(14,5)$ & $(1,1)$ & $(0,0)$ & $(0,2)$ & $(1,2)$ & $(0,1)$ & $(0,3)$ \\
\hline & $60-$ & 60,3 & 88,4 & 0,0 & 8,4 & 72,9 & 7,0 & 0,0 & 0,9 & 7,9 & 0,5 & 1,1 \\
\hline & 90 & $(5,2)$ & $(10,3)$ & $(0,0)$ & $(0,7)$ & $(7,5)$ & $(2,3)$ & $(0,0)$ & $(0,1)$ & $(1,5)$ & $(0,2)$ & $(0,1)$ \\
\hline \multirow{8}{*}{ RW } & $0-$ & 190,0 & 72,2 & 0,0 & 3,5 & 57,4 & 11,2 & 0,0 & 0,4 & 6,1 & 0,7 & 0,8 \\
\hline & 10 & $(10,9)$ & $(41,2)$ & $(0,0)$ & $(1,8)$ & $(31,3)$ & $(8,2)$ & $(0,0)$ & $(0,2)$ & $(3,4)$ & $(0,5)$ & $(0,0)$ \\
\hline & $10-$ & 193,7 & 94,1 & 0,1 & 3,2 & 71,9 & 18,9 & 0,0 & 0,7 & 16,5 & 2,6 & 1,0 \\
\hline & 30 & $(12,7)$ & $(8,8)$ & $(0,1)$ & $(1,0)$ & $(7,0)$ & $(1,3)$ & $(0,0)$ & $(0,2)$ & $(0,7)$ & $(0,1)$ & $(0,1)$ \\
\hline & $30-$ & 230,4 & 93,2 & 0,2 & 1,7 & 65,6 & 25,6 & 0,0 & 0,7 & 26,8 & 6,3 & 2,2 \\
\hline & 60 & $(5,2)$ & $(11,8)$ & $(0,1)$ & $(0,5)$ & $(5,2)$ & $(7,2)$ & $(0,0)$ & $(0,2)$ & $(1,7)$ & $(1,7)$ & $(0,1)$ \\
\hline & $60-$ & 150,6 & 100,0 & 0,9 & 1,7 & 64,5 & 32,8 & 0,1 & 0,2 & 8,1 & 2,5 & 1,5 \\
\hline & 90 & $(11,6)$ & $(0,0)$ & $(1,3)$ & $(0,3)$ & $(6,6)$ & $(5,1)$ & $(0,1)$ & $(0,1)$ & $(0,3)$ & $(0,6)$ & $(0,0)$ \\
\hline \multirow{4}{*}{ B } & $0-$ & 223,7 & 100,0 & 0,0 & 2,1 & 90,5 & 7,4 & 0,0 & 0,2 & 8,8 & 0,4 & 0,8 \\
\hline & 10 & $(17,0)$ & $(0,0)$ & $(0,0)$ & $(0,1)$ & $(1,0)$ & $(0,8)$ & $(0,0)$ & $(0,0)$ & $(0,8)$ & $(0,0)$ & $(0,0)$ \\
\hline & $10-$ & 175,4 & 100,0 & 0,2 & 2,3 & 89,7 & 7,8 & 0,0 & 0,4 & 17,1 & 0,9 & 1,7 \\
\hline & 30 & $(90,1)$ & $(0,0)$ & $(0,3)$ & $(0,5)$ & $(0,6)$ & $(0,4)$ & $(0,1)$ & $(0,2)$ & $(8,8)$ & $(0,5)$ & $(0,1)$ \\
\hline
\end{tabular}

Während die Versuchsfläche BERTA eine Basensättigung von $100 \%$ aufweist, fallen die Werte in Reiffenhausen etwas geringer aus und lassen auf leichte Versauerungstendenzen schließen.

Auf allen Flächen ist $\mathrm{Ca}^{2+}$ das am stärksten vertretene austauschbare Kation (> 51,3\%), gefolgt von $\mathrm{Mg}^{2+}(>7,0 \%), \mathrm{K}^{+}(>1,7 \%)$ und $\mathrm{Na}^{+}(<0,9 \%)$. Da Ca-haltige Minerale (Calcit, Dolomit, Gips) leicht verwitterbar sind und das zweiwertige $\mathrm{Ca}^{2+}$ im Vergleich zu einwertigen Kationen $\left(\mathrm{K}^{+}, \mathrm{Na}^{+}\right)$stärker am Austauscher gebunden wird, ist ein großer Teil der Austauscherplätze von $\mathrm{Ca}^{2+}$ dominiert. Die $\mathrm{Mg}$ Sättigung beträgt in landwirtschaftlich genutzten Böden Mitteleuropas meist 5 bis 25 \% (Blume et al. 2010). In RW kommt es durch die Verwitterung des Minerals Dolomit (Oberer Buntsandstein) zur höheren Verfügbarkeit von $\mathrm{Mg}^{2+}$ im Vergleich zum Standort BERTA. Die schwächer gebundenen einwertigen Kationen $\mathrm{Na}^{+}$und $\mathrm{K}^{+}$gehen daher leicht in Lösung.

Bezogen auf die Bodentiefe $0-90 \mathrm{~cm}$ ist die Fläche Reiffenhausen durch sehr hohe Magnesium$\left(>2 \mathrm{t}(\mathrm{Mg}) \mathrm{ha}^{-1}\right)$, Calcium- $\left(>8 \mathrm{t}(\mathrm{Ca}) \mathrm{ha}^{-1}\right)$, Kalium-Vorräte $\left(>1,6 \mathrm{t}(\mathrm{K}) \mathrm{ha}^{-1}\right)$ und hohe Phosphorvorräte $\left(>3 \mathrm{t}(\mathrm{P}) \mathrm{ha}^{-1}\right)$ gekennzeichnet. BERTA weist, bezogen auf eine Bodentiefe von 0-30 $\mathrm{cm}$, sehr hohe Calcium-, hohe Magnesium- sowie mittlere Kalium- und Phosphorvorräte auf.

Bezogen auf eine Bodentiefe von 0-90 cm in Reiffenhausen ist die Nährstoffausstattung durchweg sehr gut (mit Ausnahme von N), es werden stets die höchsten Bewertungsklassen erreicht. Der 
Boden in BERTA ist ebenfalls nährstoffreich, obwohl die Vorräte nur auf eine Mächtigkeit von $30 \mathrm{~cm}$ bezogen wurden.

\subsubsection{Feinkartierung}

Ziel der Feinkartierung war es, die Heterogenität bzw. Homogenität in der flächenhaften Verteilung der Nährstoffe zu erfassen. Da nach Jug et al. (1999b) die wesentlichen Prozesse, die zu Unterschieden in den Nährstoffen führen, im Oberboden ablaufen, wird insbesondere die Bodentiefe $0-30 \mathrm{~cm}$ betrachtet.

In Reiffenhausen konnte kein Verteilungsmuster beim $\mathrm{pH}$-Wert und dem $\mathrm{C}_{\text {org }} / \mathrm{N}_{\mathrm{t}}$-Verhältnis beobachtet werden. Hingegen konnte beim $\mathrm{N}_{\mathrm{t}^{-}}$und $\mathrm{C}_{\text {org }}$-Gehalt und somit beim Vorrat an $\mathrm{N}_{t}$ und $\mathrm{C}_{\text {org }}$ eine Zunahme von Ost ( $\mathrm{C}_{\text {org }}$-Vorrat: ca. $40 \mathrm{t}(\mathrm{C}) \mathrm{ha}^{-1}$; $\mathrm{N}_{\mathrm{t}}-$ Vorrat ca. $5 \mathrm{t}(\mathrm{N}) \mathrm{ha}^{-1}$ ) nach West $\left(\mathrm{C}_{\text {org }}\right.$-Vorrat: ca. $60 \mathrm{t}(\mathrm{C}) \mathrm{ha}^{-1} ; \mathrm{N}_{\mathrm{t}}$-Vorrat: ca. $7 \mathrm{t}(\mathrm{N}) \mathrm{ha}^{-1}$ ) festgestellt werden (Tabelle 6). Ferner wurde eine Zunahme des Tongehaltes von 8-17 \% im Osten zu 30-45 \% im Westen ermittelt. Im Westen konnten höhere Carbonatgehalte beobachtet werden. Die Vorräte an Phosphor, Kalium, Calcium und Magnesium fielen im Westen ebenfalls höher aus als im Osten (Tabelle 7).

Für die Versuchsfläche BERTA konnte kein Verteilungsmuster für die Gehalte und Vorräte an $\mathrm{C}_{\text {org }}$ und $N_{t}$ festgestellt werden (Tabelle 8). Das $C_{\text {org }} / N_{t}$-Verhältnis fiel mit 9,3 bis 10,4 homogen und eng aus. Für die Vorräte an Phosphor, Kalium, Calcium und Magnesium wurden ebenfalls keine Verteilungsmuster beobachtet (Tabelle 9).

Während auf der Versuchsfläche BERTA keine Verteilungsmuster für die Nährstoffe beobachtet wurden, wurden in Reiffenhausen stets höhere Nährstoffgehalte und Nährstoffvorräte im Westen der Fläche (Bereich KUP-Weide) festgestellt. 
Tabelle 6 Bodenchemische Kennwerte der Feinkartierung: $\mathrm{pH}-$ Wert; Gesamtgehalt und Vorrat an $\mathrm{C}_{\mathrm{org}}, \mathrm{N}_{\mathrm{t}}, \mathrm{P}_{\mathrm{t}}$, Gehalt an $\mathrm{CaMg}\left(\mathrm{CO}_{3}\right)_{2}$ und $\mathrm{C}_{\text {org }} / \mathrm{N}_{\mathrm{t}}$-Verhältnis in Reiffenhausen

\begin{tabular}{|c|c|c|c|c|c|c|c|c|c|c|c|c|}
\hline \multirow[t]{2}{*}{$\mathrm{BP}^{*}$} & \multirow[t]{2}{*}{ Lage } & \multirow{2}{*}{ Bodentyp } & \multirow{2}{*}{$\begin{array}{c}\text { Tiefe } \\
{[\mathrm{cm}]}\end{array}$} & \multirow[t]{2}{*}{$\mathrm{pH}$} & \multirow{2}{*}{$\begin{array}{c}\mathrm{C}_{\text {org }} \\
{\left[\mathrm{mg} \mathrm{g}^{-1}\right]}\end{array}$} & \multirow{2}{*}{$\begin{array}{c}\text { Corg } \\
\left.\mathrm{t} \mathrm{ha}^{-1}\right]\end{array}$} & \multirow{2}{*}{$\begin{array}{c}\text { CaMg }\left(\mathrm{CO}_{3}\right)_{2} \\
\text { Gehalt }\end{array}$} & \multirow{2}{*}{$\begin{array}{c}\mathrm{N}_{\mathrm{t}} \\
{\left[\mathrm{mg} \mathrm{g}^{-1}\right]}\end{array}$} & \multirow{2}{*}{$\begin{array}{c}\mathrm{N}_{\mathrm{t}} \\
\left.\mathrm{t} \mathrm{ha}^{-1}\right]\end{array}$} & \multirow[t]{2}{*}{$\mathrm{C}_{\text {org }} / \mathrm{N}_{\mathrm{t}}$} & \multirow{2}{*}{$\begin{array}{c}\mathrm{P}_{\mathrm{t}} \\
{\left[\mathrm{mg} \mathrm{g}^{-1}\right]}\end{array}$} & \multirow{2}{*}{$\begin{array}{c}\mathrm{P}_{\mathrm{t}} \\
\left.\mathrm{t} \mathrm{ha}^{-1}\right]\end{array}$} \\
\hline & & & & & & & & & & & & \\
\hline \multirow[t]{4}{*}{1} & AF-Weide & Para- & $0-30$ & 6,3 & 11,0 & 48,8 & 0,7 & 1,2 & 5,5 & 8,9 & 0,6 & 2,5 \\
\hline & & braunerde & $30-60$ & 6,3 & 4,2 & 18,5 & 0,4 & 0,7 & 3,0 & 6,1 & 0,4 & 1,7 \\
\hline & & & $60-90$ & 6,6 & 2,8 & 12,6 & 0,2 & 0,5 & 2,3 & 5,5 & 0,4 & 1,6 \\
\hline & & & $0-90$ & & & 79,9 & & & 9,8 & & & 5,8 \\
\hline \multirow[t]{4}{*}{2} & AF-Weide & Braunerde & $0-30$ & 5,5 & 9,1 & 40,3 & 1,1 & 1,1 & 4,9 & 8,3 & 0,5 & 2,3 \\
\hline & & & $30-60$ & 5,7 & 5,8 & 25,7 & 0,4 & 0,7 & 3,2 & 8,1 & 0,4 & 1,8 \\
\hline & & & $60-90$ & 6,2 & 2,5 & 11,3 & 0,2 & 0,4 & 1,8 & 6,2 & 0,3 & 1,5 \\
\hline & & & $0-90$ & & & 77,3 & & & 9,9 & & & 5,6 \\
\hline \multirow[t]{4}{*}{3} & AF-Weide & Braunerde- & $0-30$ & 5,9 & 10,2 & 44,5 & 1,5 & 1,3 & 5,6 & 7,9 & 0,6 & 2,8 \\
\hline & & Pseudogley & $30-60$ & 6,6 & 3,7 & 16,4 & 0,3 & 0,7 & 3,2 & 5,2 & 0,4 & 1,8 \\
\hline & & & $60-90$ & 7,1 & 2,1 & 9,6 & 0,1 & 0,5 & 2,1 & 4,6 & 0,6 & 2,7 \\
\hline & & & $0-90$ & & & 70,5 & & & 10,9 & & & 7,3 \\
\hline \multirow[t]{4}{*}{4} & KUP-Weide & Braunerde & $0-30$ & 5,9 & 13,9 & 61,8 & 0,0 & 1,0 & 4,4 & 14,2 & 0,7 & 3,1 \\
\hline & & & $30-60$ & 6,3 & 3,5 & 15,0 & 4,7 & 1,0 & 4,3 & 3,5 & 0,5 & 2,0 \\
\hline & & & $60-90$ & 6,7 & 6,6 & 29,4 & 1,3 & 1,0 & 4,4 & 6,6 & 0,6 & 2,5 \\
\hline & & & $0-90$ & & & 106,2 & & & 13,1 & & & 7,6 \\
\hline \multirow[t]{4}{*}{5} & KUP-Weide & Pelosol & $0-30$ & 6,6 & 13,7 & 59,6 & 2,6 & 1,8 & 7,6 & 7,8 & 0,7 & 3,0 \\
\hline & & & $30-60$ & 6,7 & 5,2 & 23,2 & 0,7 & 0,9 & 3,8 & 6,2 & 0,4 & 1,8 \\
\hline & & & $60-90$ & 7,8 & 2,4 & 10,1 & 5,8 & 0,6 & 2,4 & 4,3 & 0,6 & 2,6 \\
\hline & & & $0-90$ & & & 92,9 & & & 13,8 & & & 7,4 \\
\hline \multirow[t]{4}{*}{6} & KUP-Weide & Pseudo- & $0-30$ & 6,1 & 13,3 & 58,4 & 1,8 & 1,6 & 6,9 & 8,5 & 0,8 & 3,3 \\
\hline & & gley & $30-60$ & 6,8 & 4,0 & 17,7 & 0,5 & 0,7 & 3,2 & 5,6 & 0,4 & 1,8 \\
\hline & & & $60-90$ & 7,6 & 2,6 & 11,8 & 1,2 & 0,6 & 2,6 & 4,6 & 0,7 & 3,2 \\
\hline & & & $0-90$ & & & 87,9 & & & 12,7 & & & 8,3 \\
\hline \multirow[t]{4}{*}{7} & AF-Weide & Braunerde & $0-30$ & 6,7 & 8,9 & 39,0 & 2,0 & 1,1 & 4,8 & 8,1 & 0,6 & 2,6 \\
\hline & & & $30-60$ & 7,2 & 3,6 & 15,8 & 1,1 & 0,6 & 2,6 & 6,2 & 0,3 & 1,5 \\
\hline & & & $60-90$ & 7,4 & 1,1 & 5,2 & 0,0 & 0,3 & 1,3 & 4,1 & 0,1 & 0,6 \\
\hline & & & $0-90$ & & & 60,0 & & & 8,7 & & & 4,7 \\
\hline \multirow[t]{4}{*}{8} & KUP-Pappel & Braunerde & $0-30$ & 6,8 & 10,0 & 44,2 & 0,8 & 1,1 & 4,9 & 9,1 & 0,5 & 2,2 \\
\hline & & & $30-60$ & 7,2 & 3,1 & 13,6 & 0,3 & 0,5 & 2,2 & 6,2 & 0,3 & 1,5 \\
\hline & & & $60-90$ & 7,3 & 0,8 & 3,4 & 0,0 & 0,2 & 0,9 & 3,9 & 0,2 & 0,9 \\
\hline & & & $0-90$ & & & 61,2 & & & 8,0 & & & 4,6 \\
\hline \multirow[t]{4}{*}{9} & KUP-Pappel & Braunerde & $0-30$ & 6,1 & 8,9 & 39,3 & 1,3 & 1,1 & 4,7 & 8,3 & 0,5 & 2,2 \\
\hline & & & $30-60$ & 6,1 & 4,1 & 18,4 & 0,4 & 0,6 & 2,5 & 7,3 & 0,4 & 1,6 \\
\hline & & & $60-90$ & 6,7 & 0,7 & 3,2 & 0,0 & 0,2 & 0,9 & 3,5 & 0,3 & 1,5 \\
\hline & & & $0-90$ & & & 60,9 & & & 8,1 & & & 5,3 \\
\hline
\end{tabular}

*BP Beprobungspunkt 
Tabelle 7 Bodenchemische Kennwerte der Feinkartierung: Potenzielle Kationenaustauschkapazität (KAK pot $)$, Basensättigung (BS) und Kationensättigung sowie Vorrat an Natrium (Na), Kalium (K), Calcium (Ca) und Magnesium (Mg) in Reiffenhausen

\begin{tabular}{|c|c|c|c|c|c|c|c|c|c|c|c|c|}
\hline \multirow[t]{2}{*}{ BP* } & \multirow[t]{2}{*}{ Lage } & \multirow{2}{*}{$\begin{array}{l}\text { Tiefe } \\
{[\mathrm{cm}]}\end{array}$} & \multirow{2}{*}{$\frac{\mathrm{KAK}_{\mathrm{pot}}}{\left[\mathrm{mmol}_{\mathrm{c}} \mathrm{kg}^{-1}\right]}$} & \multirow{2}{*}{$\begin{array}{l}\text { BS } \\
{[\%]}\end{array}$} & \multicolumn{4}{|c|}{ Sättigung \% von KAK $K_{\text {pot }}$} & \multirow{2}{*}{$\frac{\mathrm{Na}}{\left[\mathrm{t} \mathrm{ha}^{-1}\right]}$} & \multirow{2}{*}{$\frac{\mathrm{K}}{\left[\mathrm{t} \mathrm{ha}^{-1}\right]}$} & \multirow{2}{*}{$\frac{\mathrm{Ca}}{\left[\mathrm{t} \mathrm{ha}^{-1}\right]}$} & \multirow{2}{*}{$\frac{\mathrm{Mg}}{\left[\mathrm{t} \mathrm{ha}^{-1}\right]}$} \\
\hline & & & & & $\mathrm{Na}$ & $\mathrm{K}$ & $\mathrm{Ca}$ & $\mathrm{Mg}$ & & & & \\
\hline \multirow[t]{4}{*}{1} & AF-Weide & $0-30$ & 124,3 & 72,1 & 0,0 & 7,3 & 69,7 & 5,1 & 0,0 & 1,3 & 13,2 & 0,7 \\
\hline & & $30-60$ & 101,1 & 67,3 & 0,0 & 5,6 & 55,7 & 5,9 & 0,0 & 1,0 & 10,0 & 0,6 \\
\hline & & $60-90$ & 114,9 & 73,3 & 0,0 & 3,3 & 61,5 & 8,5 & 0,0 & 0,7 & 12,8 & 1,1 \\
\hline & & $0-90$ & & & & & & & 0,0 & 3,0 & 36,0 & 2,4 \\
\hline \multirow[t]{4}{*}{2} & AF-Weide & $0-30$ & 105,9 & 53,8 & 0,0 & 8,3 & 41,4 & 4,0 & 0,0 & 1,5 & 7,8 & 0,5 \\
\hline & & $30-60$ & 103,1 & 50,6 & 0,0 & 4,9 & 42,2 & 3,5 & 0,0 & 0,9 & 7,7 & 0,4 \\
\hline & & $60-90$ & 86,5 & 61,9 & 0,2 & 2,6 & 54,9 & 4,3 & 0,0 & 0,4 & 8,6 & 0,4 \\
\hline & & $0-90$ & & & & & & & 0,0 & 2,8 & 24,1 & 1,3 \\
\hline \multirow[t]{4}{*}{3} & AF-Weide & $0-30$ & 121,8 & 68,0 & 0,0 & 5,9 & 56,5 & 5,6 & 0,0 & 1,2 & 12,1 & 0,7 \\
\hline & & $30-60$ & 183,7 & 84,3 & 0,2 & 3,3 & 66,9 & 13,9 & 0,0 & 1,1 & 21,9 & 2,8 \\
\hline & & $60-90$ & 220,5 & 96,4 & 0,3 & 1,8 & 68,3 & 26,0 & 0,1 & 0,7 & 27,3 & 6,3 \\
\hline & & $0-90$ & & & & & & & 0,1 & 3,0 & 61,3 & 9,8 \\
\hline \multirow[t]{4}{*}{4} & KUP-Weide & $0-30$ & 172,6 & 75,0 & 0,0 & 5,1 & 60,7 & 9,2 & 0,0 & 1,5 & 18,7 & 1,7 \\
\hline & & $30-60$ & 163,5 & 79,1 & 0,1 & 2,3 & 66,4 & 10,2 & 0,0 & 0,6 & 18,5 & 1,7 \\
\hline & & $60-90$ & 218,5 & 92,8 & 0,3 & 1,4 & 74,7 & 16,4 & 0,1 & 0,5 & 29,3 & 3,9 \\
\hline & & $0-90$ & & & & & & & 0,1 & 2,6 & 66,5 & 7,3 \\
\hline \multirow[t]{4}{*}{5} & KUP-Weide & $0-30$ & 191,7 & 84,4 & 0,0 & 6,1 & 63,9 & 14,4 & 0,0 & 2,0 & 21,3 & 2,9 \\
\hline & & $30-60$ & 198,3 & 100,0 & 0,3 & 2,8 & 72,5 & 24,4 & 0,1 & 1,0 & 25,5 & 5,2 \\
\hline & & $60-90$ & 216,1 & 100,0 & 0,4 & 1,1 & 68,4 & 30,1 & 0,1 & 0,4 & 25,2 & 6,7 \\
\hline & & $0-90$ & & & & & & & 0,2 & 3,4 & 72,0 & 14,8 \\
\hline \multirow[t]{4}{*}{6} & KUP-Weide & $0-30$ & 143,9 & 74,1 & 0,0 & 6,5 & 60,7 & 7,0 & 0,0 & 1,6 & 15,3 & 1,1 \\
\hline & & $30-60$ & 217,9 & 99,1 & 0,1 & 2,9 & 82,8 & 13,2 & 0,0 & 1,1 & 32,1 & 3,1 \\
\hline & & $60-90$ & 234,1 & 100,0 & 0,2 & 1,4 & 84,8 & 13,6 & 0,1 & 0,6 & 35,6 & 3,5 \\
\hline & & $0-90$ & & & & & & & 0,1 & 3,3 & 83,0 & 7,7 \\
\hline \multirow[t]{4}{*}{7} & AF-Weide & $0-30$ & 94,7 & 81,6 & 0,0 & 8,9 & 69,1 & 3,7 & 0,0 & 1,4 & 11,4 & 0,4 \\
\hline & & $30-60$ & 76,9 & 83,2 & 0,0 & 3,8 & 77,9 & 1,5 & 0,0 & 0,5 & 10,6 & 0,1 \\
\hline & & $60-90$ & 55,3 & 86,4 & 0,0 & 5,7 & 76,2 & 4,5 & 0,0 & 0,6 & 7,7 & 0,3 \\
\hline & & $0-90$ & & & & & & & 0,0 & 2,5 & 29,7 & 0,8 \\
\hline \multirow[t]{4}{*}{8} & KUP-Pappel & $0-30$ & 105,5 & 91,8 & 0,0 & 6,5 & 81,2 & 4,2 & 0,0 & 1,2 & 15,2 & 0,5 \\
\hline & & $30-60$ & 81,5 & 86,2 & 0,0 & 5,2 & 78,6 & 2,4 & 0,0 & 0,7 & 11,4 & 0,2 \\
\hline & & $60-90$ & 52,0 & 88,3 & 0,0 & 7,4 & 75,6 & 5,3 & 0,0 & 0,7 & 7,1 & 0,3 \\
\hline & & $0-90$ & & & & & & & 0,0 & 2,6 & 33,7 & 1,0 \\
\hline \multirow[t]{4}{*}{9} & KUP-Pappel & $0-30$ & 96,5 & 67,5 & 0,0 & 9,0 & 54,2 & 4,2 & 0,0 & 1,5 & 9,2 & 0,4 \\
\hline & & $30-60$ & 78,9 & 68,3 & 0,0 & 5,2 & 58,5 & 4,6 & 0,0 & 0,7 & 8,2 & 0,4 \\
\hline & & $60-90$ & 46,8 & 70,8 & 0,0 & 7,3 & 57,5 & 6,0 & 0,0 & 0,6 & 4,9 & 0,3 \\
\hline & & $0-90$ & & & & & & & 0,0 & 2,8 & 22,3 & 1,1 \\
\hline
\end{tabular}

*BP Beprobungspunkt 
Tabelle 8 Bodenchemische Kennwerte der Feinkartierung: pH-Wert; Gesamtgehalt und Vorrat an $\mathrm{C}_{\text {org }}, \mathrm{N}_{\mathrm{t}}, \mathrm{P}_{\mathrm{t}}$, Gehalt an $\mathrm{CaCO}_{3}$ und $\mathrm{C}_{\text {org }} / \mathrm{N}_{\mathrm{t}}$-Verhältnis in BERTA

\begin{tabular}{|c|c|c|c|c|c|c|c|c|c|c|c|c|}
\hline \multirow[t]{2}{*}{ BP* } & \multirow[t]{2}{*}{ Lage } & \multirow[t]{2}{*}{ Bodentyp } & \multirow{2}{*}{$\begin{array}{c}\text { Tiefe } \\
{[\mathrm{cm}]}\end{array}$} & \multirow[t]{2}{*}{$\mathrm{pH}$} & \multirow{2}{*}{$\begin{array}{c}\mathrm{C}_{\text {org }} \\
{\left[\mathrm{mg} \mathrm{g}^{-1}\right]}\end{array}$} & \multirow{2}{*}{$\begin{array}{c}\mathrm{C}_{\text {org }} \\
\left.\mathrm{t} \mathrm{ha}^{-1}\right]\end{array}$} & \multirow{2}{*}{$\begin{array}{c}\mathrm{CaCO}_{3} \\
\text { Gehalt } \\
{[\%]}\end{array}$} & \multirow{2}{*}{$\begin{array}{c}\mathrm{N}_{\mathrm{t}} \\
{\left[\mathrm{mg} \mathrm{g}^{-1}\right]}\end{array}$} & \multirow{2}{*}{$\begin{array}{c}\mathrm{N}_{\mathrm{t}} \\
{\left[\mathrm{t} \mathrm{ha}^{-1}\right]}\end{array}$} & \multirow[t]{2}{*}{$\mathrm{C}_{\text {org }} / \mathrm{N}_{\mathrm{t}}$} & \multirow{2}{*}{$\begin{array}{c}\mathrm{P}_{\mathrm{t}} \\
{\left[\mathrm{mg} \mathrm{g}^{-1}\right]}\end{array}$} & \multirow{2}{*}{$\begin{array}{c}\mathrm{P}_{\mathrm{t}} \\
\left.\mathrm{t} \mathrm{ha}^{-1}\right]\end{array}$} \\
\hline & & & & & & & & & & & & \\
\hline \multirow[t]{4}{*}{1} & KUP-Weide & Kolluvisol & $0-30$ & & 14,1 & 47,3 & 5,2 & 1,5 & 5,1 & 9,3 & 0,8 & 2,7 \\
\hline & & & $30-60$ & 8,0 & 10,8 & 18,0 & 5,1 & 1,3 & 2,2 & 8,1 & 0,6 & 1,0 \\
\hline & & & $60-90$ & 7,9 & 10,4 & 18,5 & 2,3 & 1,1 & 2,0 & 9,4 & 0,4 & 0,8 \\
\hline & & & $0-90$ & & & 83,8 & & & 9,3 & & & 4,5 \\
\hline \multirow[t]{4}{*}{2} & KUP-Weide & Kolluvisol & $0-30$ & & 13,4 & 45,8 & 3,2 & 1,4 & 4,6 & 9,9 & 0,7 & 2,5 \\
\hline & & & $30-60$ & 8,1 & 11,1 & 19,1 & 3,9 & 1,4 & 2,3 & 8,1 & 0,6 & 1,0 \\
\hline & & & $60-90$ & 8,0 & 13,8 & 23,2 & 5,0 & 1,5 & 2,5 & 9,3 & 0,5 & 0,8 \\
\hline & & & $0-90$ & & & 88,1 & & & 9,4 & & & 4,3 \\
\hline \multirow[t]{4}{*}{3} & KUP-Weide & Kolluvisol & $0-30$ & 7,5 & 15,2 & 52,0 & 3,1 & 1,6 & 5,5 & 9,4 & 0,6 & 2,0 \\
\hline & & & $30-60$ & 8,0 & 12,3 & 21,6 & 2,8 & 1,4 & 2,5 & 8,8 & 0,5 & 0,9 \\
\hline & & & $60-90$ & 8,3 & 17,0 & 28,6 & 4,8 & 1,8 & 3,0 & 9,4 & 0,5 & 0,9 \\
\hline & & & $0-90$ & & & 102,2 & & & 11 & & & 3,8 \\
\hline \multirow[t]{4}{*}{4} & Acker & Braunerde & $0-30$ & 7,2 & 13,8 & 47,6 & 1,5 & 1,5 & 5,0 & 9,5 & 0,6 & 2,0 \\
\hline & & & $30-60$ & 7,8 & 10,7 & 19,1 & 2,3 & 1,2 & 2,2 & 8,6 & 0,5 & 0,9 \\
\hline & & & $60-90$ & 8,2 & 10,9 & 19,8 & 1,6 & 1,1 & 2,0 & 10,1 & 0,4 & 0,7 \\
\hline & & & $0-90$ & & & 86,5 & & & 9,2 & & & 3,6 \\
\hline \multirow[t]{4}{*}{5} & AF-GL & Parabraunerde & $0-30$ & 7,8 & & & & 1,3 & 4,7 & & 0,5 & 1,8 \\
\hline & & & $30-60$ & 7,8 & 11,9 & 21,3 & 2,0 & 1,2 & 2,2 & 9,7 & 0,4 & 0,8 \\
\hline & & & $60-90$ & 8,3 & 5,2 & 5,8 & 20,4 & 0,7 & 0,8 & 7,5 & 0,6 & 0,6 \\
\hline & & & $0-90$ & & & & & & 7,7 & & & 3,2 \\
\hline \multirow[t]{4}{*}{6} & Acker & Pararendzina & $0-30$ & & & & & 1,5 & 5,2 & & 0,6 & 2,1 \\
\hline & & & $30-60$ & 8,1 & 11,8 & 14,7 & 16,7 & 1,4 & 1,8 & 8,2 & 0,7 & 0,8 \\
\hline & & & $60-90$ & 8,6 & 2,4 & 0,0 & 61,3 & 0,3 & 0,0 & 7,9 & 0,8 & 0,0 \\
\hline & & & $0-90$ & & & & & & 7,0 & & & 2,9 \\
\hline \multirow[t]{4}{*}{7} & AF-Weide & Parabraunerde & $0-30$ & & 13,1 & 45,5 & 1,7 & 1,3 & 4,4 & 10,4 & 0,5 & 1,8 \\
\hline & $\mathrm{AF}$ & & $30-60$ & 7,9 & 12,3 & 22,3 & 1,4 & 1,3 & 2,3 & 9,8 & 0,4 & 0,7 \\
\hline & & & $60-90$ & 8,4 & 8,1 & 13,7 & 4,8 & 0,9 & 1,4 & 9,5 & 0,4 & 0,7 \\
\hline & & & $0-90$ & & & 81,5 & & & 8,1 & & & 3,2 \\
\hline \multirow[t]{4}{*}{8} & KUP-Pappel & Parabraunerde & $0-30$ & 7,9 & & & & 1,3 & 4,4 & & 0,5 & 1,9 \\
\hline & & & $30-60$ & 8,2 & 8,9 & 14,3 & 7,1 & 1,1 & 1,7 & 8,3 & 0,5 & 0,8 \\
\hline & & & $60-90$ & 8,4 & 3,8 & 2,8 & 30,4 & 0,5 & 0,4 & 7,0 & 0,7 & 0,5 \\
\hline & & & $0-90$ & & & & & & 6,5 & & & 3,2 \\
\hline \multirow[t]{4}{*}{9} & AF-Weide & Parabraunerde & $0-30$ & & 13,5 & 46,7 & 2,3 & 1,3 & 4,6 & 10,2 & 0,6 & 1,9 \\
\hline & & & $30-60$ & 8,3 & 12,2 & 21,0 & 3,6 & 1,2 & 2,1 & 10,1 & 0,5 & 0,8 \\
\hline & & & $60-90$ & 8,3 & 4,0 & 4,5 & 20,1 & 0,7 & 0,7 & 6,1 & 0,7 & 0,8 \\
\hline & & & $0-90$ & & & 72,2 & & & 7,4 & & & 3,5 \\
\hline
\end{tabular}

*BP Beprobungspunkt, leere Zeilen ergeben sich durch zu wenig Bodenmaterial 
Tabelle 9 Bodenchemische Kennwerte der Feinkartierung: Potenzielle Kationenaustauschkapazität (KAK pot $)$, Basensättigung (BS) und Kationensättigung sowie Vorrat an Natrium (Na), Kalium (K), Calcium (Ca) und Magnesium (Mg) in BERTA

\begin{tabular}{|c|c|c|c|c|c|c|c|c|c|c|c|c|}
\hline \multirow[t]{2}{*}{ BP } & \multirow[t]{2}{*}{ Lage } & \multirow{2}{*}{$\begin{array}{l}\text { Tiefe } \\
{[\mathrm{cm}]}\end{array}$} & \multirow{2}{*}{$\frac{\mathrm{KAK}_{\mathrm{pot}}}{\left[\mathrm{mmol}_{\mathrm{c}} \mathrm{kg}^{-1}\right]}$} & \multirow{2}{*}{$\begin{array}{l}\text { BS } \\
{[\%]}\end{array}$} & \multicolumn{4}{|c|}{ Sättigung \% von $\mathrm{KAK}_{\text {pot }}$} & \multirow{2}{*}{$\frac{\mathrm{Na}}{\left[\mathrm{t} \mathrm{ha}^{-1}\right]}$} & \multirow{2}{*}{$\frac{\mathrm{K}}{\left[\mathrm{t} \mathrm{ha}^{-1}\right]}$} & \multirow{2}{*}{$\frac{\mathrm{Ca}}{\left[\mathrm{t} \mathrm{ha}^{-1}\right]}$} & \multirow{2}{*}{$\frac{\mathrm{Mg}}{\left[\mathrm{t} \mathrm{ha}^{-1}\right]}$} \\
\hline & & & & & $\mathrm{Na}$ & K & $\mathrm{Ca}$ & $\mathrm{Mg}$ & & & & \\
\hline \multirow[t]{4}{*}{1} & KUP-Weide & $0-30$ & 206,1 & 100,0 & 0,7 & 4,6 & 81,1 & 13,5 & 0,1 & 1,2 & 22,4 & 2,3 \\
\hline & & $30-60$ & 201,7 & 99,3 & 0,9 & 3,0 & 81,3 & 14,0 & 0,1 & 0,4 & 11,0 & 1,2 \\
\hline & & $60-90$ & 197,3 & 100,0 & 1,1 & 1,4 & 82,5 & 15,1 & 0,1 & 0,2 & 11,6 & 1,3 \\
\hline & & $0-90$ & & & & & & & 0,3 & 1,8 & 45,0 & 4,8 \\
\hline \multirow[t]{4}{*}{2} & KUP-Weide & $0-30$ & 217,4 & 100,0 & 1,0 & 1,7 & 83,5 & 13,8 & 0,2 & 0,5 & 24,9 & 2,5 \\
\hline & & $30-60$ & 210,8 & 100,0 & 1,0 & 1,9 & 82,3 & 14,9 & 0,1 & 0,3 & 11,9 & 1,3 \\
\hline & & $60-90$ & 233,0 & 100,0 & 0,8 & 1,2 & 85,0 & 13,0 & 0,1 & 0,2 & 13,3 & 1,2 \\
\hline & & $0-90$ & & & & & & & 0,4 & 1,0 & 50,1 & 5,0 \\
\hline \multirow[t]{4}{*}{3} & KUP-Weide & $0-30$ & 215,4 & 100,0 & 0,7 & 2,1 & 79,7 & 17,4 & 0,1 & 0,6 & 23,6 & 3,1 \\
\hline & & $30-60$ & 207,2 & 100,0 & 0,9 & 1,7 & 79,9 & 17,5 & 0,1 & 0,2 & 11,7 & 1,6 \\
\hline & & $60-90$ & 243,9 & 100,0 & 0,8 & 1,3 & 82,1 & 15,8 & 0,1 & 0,2 & 13,5 & 1,6 \\
\hline & & $0-90$ & & & & & & & 0,3 & 1,0 & 48,8 & 6,3 \\
\hline \multirow[t]{4}{*}{4} & Acker & $0-30$ & 199,6 & 100,0 & 0,7 & 1,9 & 78,4 & 19,0 & 0,1 & 0,5 & 21,6 & 3,2 \\
\hline & & $30-60$ & 209,4 & 89,0 & 1,0 & 1,4 & 70,1 & 16,5 & 0,1 & 0,2 & 10,5 & 1,5 \\
\hline & & $60-90$ & 203,2 & 100,0 & 1,1 & 1,0 & 83,8 & 14,1 & 0,1 & 0,1 & 12,3 & 1,3 \\
\hline & & $0-90$ & & & & & & & 0,3 & 0,8 & 44,4 & 6,0 \\
\hline \multirow[t]{4}{*}{5} & AF-GL & $0-30$ & 201,0 & 100,0 & 0,7 & 1,3 & 86,6 & 11,4 & 0,1 & 0,4 & 24,7 & 2,0 \\
\hline & & $30-60$ & 194,8 & 95,8 & 1,0 & 1,1 & 84,2 & 9,5 & 0,1 & 0,2 & 11,7 & 0,8 \\
\hline & & $60-90$ & 198,1 & 100,0 & 1,1 & 1,4 & 85,6 & 11,9 & 0,1 & 0,1 & 7,5 & 0,6 \\
\hline & & $0-90$ & & & & & & & 0,3 & 0,7 & 43,9 & 3,4 \\
\hline \multirow[t]{4}{*}{6} & Acker & $0-30$ & 201,1 & 100,0 & 1,8 & 2,3 & 89,0 & 6,9 & 0,3 & 0,6 & 25,4 & 1,2 \\
\hline & & $30-60$ & 182,4 & 94,6 & 0,7 & 1,8 & 86,4 & 5,7 & 0,0 & 0,2 & 7,8 & 0,3 \\
\hline & & $60-90$ & 112,3 & 100,0 & 1,3 & 1,8 & 92,7 & 4,2 & 0,0 & 0,0 & 0,0 & 0,0 \\
\hline & & $0-90$ & & & & & & & 0,3 & 0,8 & 33,2 & 1,5 \\
\hline \multirow[t]{4}{*}{7} & AF-Weide & $0-30$ & 176,2 & 100,0 & 1,2 & 1,4 & 88,1 & 9,4 & 0,2 & 0,3 & 21,6 & 1,4 \\
\hline & AF & $30-60$ & 221,0 & 85,0 & 0,9 & 1,1 & 75,8 & 7,3 & 0,1 & 0,2 & 12,1 & 0,7 \\
\hline & & $60-90$ & 185,3 & 100,0 & 1,0 & 1,3 & 89,7 & 8,1 & 0,1 & 0,2 & 11,2 & 0,6 \\
\hline & & $0-90$ & & & & & & & 0,4 & 0,8 & 44,9 & 2,7 \\
\hline \multirow[t]{4}{*}{8} & KUP-Pappel & $0-30$ & 184,7 & 100,0 & 0,9 & 1,8 & 89,0 & 8,2 & 0,1 & 0,5 & 23,3 & 1,3 \\
\hline & & $30-60$ & 186,8 & 100,0 & 1,1 & 1,6 & 89,6 & 7,6 & 0,1 & 0,2 & 10,7 & 0,6 \\
\hline & & $60-90$ & 140,9 & 100,0 & 1,1 & 1,9 & 89,2 & 7,8 & 0,0 & 0,1 & 3,7 & 0,2 \\
\hline & & $0-90$ & & & & & & & 0,2 & 0,8 & 37,7 & \\
\hline \multirow[t]{4}{*}{9} & AF-Weide & $0-30$ & 216,1 & 100,0 & 1,7 & 2,1 & 82,3 & 13,8 & 0,3 & 0,6 & 24,7 & 2,5 \\
\hline & & $30-60$ & 211,5 & 100,0 & 0,9 & 1,4 & 82,7 & 15,0 & 0,1 & 0,2 & 12,1 & 1,3 \\
\hline & & $60-90$ & 219,2 & 100,0 & 0,8 & 1,3 & 81,4 & 16,5 & 0,0 & 0,1 & 8,0 & 1,0 \\
\hline & & $0-90$ & & & & & & & 0,4 & 0,9 & 44,2 & 4,8 \\
\hline
\end{tabular}

BP Beprobungspunkt 
Der Vergleich der Nährstoffgehalte und -Vorräte der Leitprofile mit den Werten der Feinkartierung soll Aussagen liefern, inwieweit die Leitprofilproben dem flächenhaften Mittel der Feinkartierung entsprechen. Die Ergebnisse der Feinkartierung und signifikante Unterschiede zwischen dem Leitprofil und der Feinkartierung sind in Tabelle 10 aufgeführt.

Das Leitprofil Reiffenhausen (Mittelwert aus RR und RW) weist im Tiefenbereich $(0-60 \mathrm{~cm}) \mathrm{im}$ Vergleich zur flächenhaften Feinkartierung signifikant geringere organische Kohlenstoff- und Gesamtstickstoffgehalte auf (Tabelle 10). Dies lässt darauf schließen, dass die Vorräte für beide Tiefenstufen unterschätzt wurden. Unter Berücksichtigung der Flächenmittelwerte an $\mathrm{C}_{\text {org }}$ und $\mathrm{N}_{t}$ der Feinkartierung ergeben sich "mittlere" $C_{\text {org-Vorräte von }} 77 \mathrm{t}$ (C) ha ${ }^{-1}$ und $\mathrm{N}_{\mathrm{t}}$-Vorräte von 10,7 t (N) ha ${ }^{-1}$ für die Bodentiefe 0-90 cm in Reiffenhausen. Da der $\mathrm{N}_{\mathrm{t}}$-Vorrat aus der Feinkartierung mit 10,7 t (N) $\mathrm{ha}^{-1}$ im Vergleich zur Leitprofilbeprobung mit 7,3-8,1 t $(\mathrm{N}) \mathrm{ha}^{-1}$ signifikant höher ausfiel, wird in der Bewertung nach Tabelle 3 nun die Bewertungsklasse "hoch" erreicht, d.h. über die Fläche gemittelt weist der Standort Reiffenhausen hohe Vorräte an Stickstoff auf. Für BERTA wurden bei der flächenhaften Beprobung signifikant geringere $C_{\text {org }}$-Gehalte im Vergleich zum Leitprofil ermittelt, sodass die $\mathrm{C}_{\text {org }}$-Vorräte im Ap-Horizont mit $59 \mathrm{t}$ (C) ha ${ }^{-1}$ überschätzt wurden. Über die Fläche verteilt ergibt sich ein mittlerer $\mathrm{C}_{\text {org }}$-Vorrat von ca. $48 \mathrm{t}$ (C) ha ${ }^{-1}$; dies entspricht der Bewertungsklasse "sehr gering" ( $\mathrm{C}_{\text {org }}$-Vorrat $\left.<50 \mathrm{t}(\mathrm{C}) \mathrm{ha}^{-1}\right)$.

Tabelle 10 Mittlere (Standardabweichung) bodenchemische Kennwerte der Feinkartierung ( $n=9)$ : pH-Wert; Gehalt und Vorrat an $\mathrm{C}_{\text {org, }} \mathrm{N}_{\mathrm{t}}$, Gehalt an $\mathrm{CaMg}\left(\mathrm{CO}_{3}\right)_{2}$ und $\mathrm{CaCO}_{3}$ sowie $\mathrm{C}_{\text {org }} / \mathrm{N}_{\mathrm{t}}$-Verhältnis der Flächen Reiffenhausen (R) und BERTA (B)

\begin{tabular}{|c|c|c|c|c|c|c|c|c|}
\hline \multirow[t]{2}{*}{ Fläche } & Tiefe & $\mathrm{pH}$ & $\mathrm{C}_{\text {org }}$ & $\mathrm{C}_{\text {org }}$ & $\mathrm{CaMg}\left(\mathrm{CO}_{3}\right)_{2}, \mathrm{CaCO}_{3}{ }^{*}$ & $\mathrm{~N}_{\mathrm{t}}$ & $\mathrm{N}_{\mathrm{t}}$ & $\mathrm{C}_{\text {org }} / \mathrm{N}_{\mathrm{t}}$ \\
\hline & [cm] & & {$\left[\mathrm{mg} \mathrm{g}^{-1}\right]$} & {$\left[\mathrm{t} \mathrm{ha}^{-1}\right]$} & [\%] & {$\left[\mathrm{mg} \mathrm{g}^{-1}\right]$} & {$\left[\mathrm{t} \mathrm{ha}^{-1}\right]$} & \\
\hline \multirow[t]{3}{*}{$\mathrm{R}$} & $0-30$ & $6,2(0,4)$ & $11,0^{\wedge}(2,1)$ & $48,4^{\wedge}(9,2)$ & $1,3(0,8)$ & $1,2^{\wedge}(0,3)$ & $5,5^{\wedge}(1,1)$ & $9,0(2,0)$ \\
\hline & $30-60$ & $6,5(0,5)$ & $4,1^{\wedge}(0,9)$ & $18,3^{\wedge}(3,9)$ & $1,0(1,4)$ & $0,7^{\wedge}(0,2)$ & $3,1^{\wedge}(0,6)$ & $6,0(1,3)$ \\
\hline & $60-90$ & $7,0(0,5)$ & $2,4(1,8)$ & $10,7(7,9)$ & $1,0^{\wedge}(1,9)$ & $0,5($ & $2,1(1,1)$ & $4,8(1,1)$ \\
\hline \multirow[t]{3}{*}{ B } & $0-30$ & $7,6(0,3)$ & $13,8^{\wedge}(0,7)$ & $47,5^{\wedge}(2,4)$ & $3,0(1,2)$ & $1,4(0,1)$ & $4,8(0,4)$ & $9,8(0,4)$ \\
\hline & $30-60$ & $8,0(0,2)$ & $11,3(1,1)$ & $19,0(2,9)$ & $5,0(4,7)$ & $1,3(0,1)$ & $2,1(0,2)$ & $8,9(0,9)$ \\
\hline & $60-90$ & $8,3(0,2)$ & $8,4(5,0)$ & $13,0(10,1)$ & $16,7(19,6)$ & $1,0(0,5)$ & $1,4(1,0)$ & $8,9(1,4)$ \\
\hline
\end{tabular}

${ }^{*} \mathrm{CaMg}\left(\mathrm{CO}_{3}\right)_{2}$ in Reiffenhausen, $\mathrm{CaCO}_{3}$ in BERTA

$\wedge^{\wedge}$ sign. Unterschiede zwischen dem Leitprofil und dem Flächenmittel, $p \leq 0,05$

BERTA: hier wurden signifikante Unterschiede nur für die Tiefenstufe $0-30 \mathrm{~cm}$ bestimmt

In Reiffenhausen konnten für den Oberboden (0-30 cm Bodentiefe) signifikante Unterschiede bei den Kalium-, Magnesium- und Phosphormengen beobachtet werden (Tabelle 11). Während die Menge an Phosphor und Kalium beim Leitprofil im Vergleich zur Feinkartierung unterschätzt wurde, wurde die Menge an Magnesium überschätzt.

Unter Berücksichtigung der Nährstoffvorräte aus der Feinkartierung (Bodentiefe 0-90 cm, Tabelle 7, Tabelle 11) ergeben sich für Reiffenhausen "sehr hohe" Magnesium- $\left(>2 \mathrm{t}(\mathrm{Mg}) \mathrm{ha}^{-1}\right)$, Calcium- $(>8 \mathrm{t}$ (Ca) ha $\left.{ }^{-1}\right)$ und Kalium-Vorräte $\left(>0,4 \mathrm{t}(\mathrm{K}) \mathrm{ha}^{-1}\right)$. Die Phosphorvorräte sind mit 6,3 t $(\mathrm{P}) \mathrm{ha}^{-1}$, bezogen 
auf die gleiche Bodentiefe, "hoch". Beim Vergleich der Nährstoffvorräte zwischen Feinkartierung und Leitprofil trat keine Änderung in der Bewertungsklasse der Nährstoffvorräte auf.

In BERTA konnten signifikante Unterschiede zwischen den Nährstoffmengen der Feinkartierung und dem Leitprofil für Calcium, Magnesium und Phosphor im Oberboden $(0-30 \mathrm{~cm})$ beobachtet werden (Tabelle 11). Vergleicht man die Bewertung der Vorräte aus dem Leitprofil und der Feinkartierung, ergeben sich keine Änderungen in den Bewertungsklassen für Calcium und Phosphor. Da die Magnesiummenge beim Leitprofil im Vergleich zur flächenhaften Kartierung signifikant unterschätzt wurde, muss die Bewertungsklasse von "hoch" auf "sehr hoch" korrigiert werden.

Tabelle 11 Mittlere (Standardabweichung) bodenchemische Kennwerte der Feinkartierung ( $n=9)$ : Potenzielle Kationenaustauschkapazität (KAK $\mathrm{Kot}_{\mathrm{t}}$ ), Basensättigung (BS) und Kationensättigung sowie Vorrat an Natrium (Na), Kalium (K), Calcium (Ca), Magnesium (Mg) und Gesamtphosphor $\left(P_{t}\right)$ der Flächen Reiffenhausen (R) und BERTA (B)

\begin{tabular}{|c|c|c|c|c|c|c|c|c|c|c|c|c|}
\hline \multirow[t]{2}{*}{ Fläche } & \multirow{2}{*}{$\begin{array}{l}\text { Tiefe } \\
{[\mathrm{cm}]} \\
\end{array}$} & \multirow{2}{*}{$\frac{\mathrm{KAK}_{\text {pot }}}{\left[\mathrm{mmol}_{\mathrm{c}} \mathrm{kg}^{-1}\right]}$} & \multirow{2}{*}{$\begin{array}{c}\text { BS } \\
{[\%]} \\
\end{array}$} & \multicolumn{4}{|c|}{ Sättigung \% von KAK $\mathrm{Kot}$} & \multirow{2}{*}{$\frac{\mathrm{Na}}{\left[\mathrm{t} \mathrm{ha}^{-1}\right]}$} & \multirow{2}{*}{$\frac{\mathrm{K}}{\left[\mathrm{t} \mathrm{ha}^{-1}\right]}$} & \multirow{2}{*}{$\frac{\mathrm{Ca}}{\left[\mathrm{t} \mathrm{ha}^{-1}\right]}$} & \multirow{2}{*}{$\frac{\mathrm{Mg}}{\left[\mathrm{t} \mathrm{ha}^{-1}\right]}$} & \multirow{2}{*}{$\frac{\mathrm{P}_{\mathrm{t}}}{\left[\mathrm{t} \mathrm{ha}^{-1}\right]}$} \\
\hline & & & & $\mathrm{Na}$ & $\mathrm{K}$ & $\mathrm{Ca}$ & $\mathrm{Mg}$ & & & & & \\
\hline \multirow{6}{*}{$\mathrm{R}$} & $0-$ & $1285(241)$ & 74,3 & 0,0 & 7,1 & 60,8 & 6,4 & 0,0 & $1,5^{\wedge}$ & 13,8 & $1,0^{\wedge}$ & $2,7^{\wedge}$ \\
\hline & 30 & $128,3(34,4)$ & $(11,0)$ & $(0,0)$ & $(1,4)$ & $(10,8)$ & $(3,5)$ & $(0,0)$ & $(0,2)$ & $(4,3)$ & $(0,8)$ & $(0,4)$ \\
\hline & $30-$ & & 79,8 & 0,1 & 4,0 & 66,8 & 8,9 & 0,0 & 0,8 & 16,2 & $1,6^{\wedge}$ & 1,7 \\
\hline & 60 & $133,9(56,0)$ & $(15,8)$ & $(0,1)$ & $(1,2)$ & $(13,0)$ & $(7,4)$ & $(0,0)$ & $(0,2)$ & $(8,7)$ & $(1,7)$ & $(0,2)$ \\
\hline & $60-$ & & 85,6 & 0,2 & 3,6 & 69,1 & 12,7 & 0,0 & 0,6 & $17,6^{\wedge}$ & 2,5 & 1,9 \\
\hline & 90 & $138,3(82,4)$ & $(13,8)$ & $(0,2)$ & $(2,6)$ & $(9,8)$ & $(9,7)$ & $(0,0)$ & $(0,1)$ & $(11,7)$ & $(2,7)$ & $(0,9)$ \\
\hline \multirow{6}{*}{ B } & $0-$ & ו2 14) 202 & 100,0 & 1,0 & 2,1 & 84,2 & 12,6 & $0,2^{\wedge}$ & 0,6 & $23,6^{\wedge}$ & $2,2^{\wedge}$ & $2,1^{\wedge}$ \\
\hline & 30 & $\angle U<, U(14, \angle)$ & $(0,0)$ & $(0,4)$ & $(1,0)$ & $(4,1)$ & $(4,1)$ & $(0,1)$ & $(0,3)$ & $(1,4)$ & $(0,8)$ & $(0,3)$ \\
\hline & 30- & & 96,0 & 0,9 & 1,7 & 81,4 & 12,0 & 0,1 & 0,2 & 11,1 & 1,0 & 0,9 \\
\hline & 60 & $202,8(12,6)$ & $(5,5)$ & $(0,1)$ & $(0,6)$ & $(5,7)$ & $(4,5)$ & $(0,0)$ & $(0,1)$ & $(1,4)$ & $(0,4)$ & $(0,1)$ \\
\hline & $60-$ & & 100,0 & 1,0 & 1,4 & 85,8 & 11,8 & 0,1 & 0,1 & 9,0 & 0,9 & 0,6 \\
\hline & 90 & $192,6(42,3)$ & $(0,0)$ & $(0,2)$ & $(0,3)$ & $(3,9)$ & $(4,2)$ & $(0,0)$ & $(0,1)$ & $(4,7)$ & $(0,5)$ & $(0,3)$ \\
\hline
\end{tabular}

$\wedge$ sign. Unterschiede zwischen dem Leitprofil und dem Flächenmittel, $p \leq 0,05$

BERTA: signifikante Unterschiede nur für die Tiefenstufe $0-30 \mathrm{~cm}$ bestimmt

Keine Überprüfung auf signifikante Unterschiede bei der Sättigung \% von KAK $_{\text {pot }}$

Signifikante Unterschiede in den Nährstoffgehalten und Nährstoffmengen zwischen den Leitprofilen und der Feinkartierung konnten hauptsächlich im Oberboden nachgewiesen werden. Diese führten jedoch kaum zu einer Änderung in der Nährstoffbewertungsklasse. Es konnte sowohl mit dem Leitprofil als auch mit der Feinkartierung stets der Reichtum an Nährstoffen für die landwirtschaftlichen Flächen Reiffenhausen und BERTA nachgewiesen werden.

\subsection{N-Kreislauf, -Vorräte und -Flüsse}

\subsubsection{Extrahierbarer mineralischer Stickstoff}

In Reiffenhausen konnte von Oktober 2011 bis September 2012 im Flächenmittel eine jährlich extrahierbare mineralische Stickstoffmenge (eIN) von $18 \mathrm{~kg}(\mathrm{~N}) \mathrm{ha}^{-1}$ beobachtet werden. Über den gesamten Zeitraum hinweg wurden im Mittel die höchsten Mengen auf der Teilfläche Acker mit insgesamt $28,5 \mathrm{~kg}(\mathrm{~N}) \mathrm{ha}^{-1}$ ermittelt (Tabelle 12). Die geringsten elN-Werte wurden auf der Teilfläche AF-Grünland mit ca. $6,6 \mathrm{~kg}(\mathrm{~N}) \mathrm{ha}^{-1}$ beobachtet. Auf dieser Fläche dominiert als Stickstoffform mit ca. 
$56 \%$ Ammonium (elN-NH mehr als $70 \%$.

In Abb. 11 wird der Verlauf der monatlich mittleren elN-Mengen für die verschiedenen Teilflächen in Reiffenhausen dargestellt. Auf der Ackerfläche wurde im August 2011 Winterraps ausgebracht. Die Monate Oktober und November 2011 zeichneten sich durch eine im Vergleich zum langjährigen Mittel milde und trockene Witterung aus (Abb. 5). Ab Ende November bzw. Anfang Dezember 2011 fielen kräftige Niederschläge, die Temperatur lag dabei deutlich über dem langjährigen Mittel (Abb. 5). Demzufolge konnte der Winterraps kräftig an Masse zulegen. Damit verbunden nahm auch die Menge an eIN kontinuierlich ab (Herold u. Höpfner 2008) (Abb. 11). Erst ab Ende Dezember 2011 bzw. Anfang Januar 2012 setzte beim Winterraps die vollständige Vegetationsruhe ein (Eiler u. Matuschek 2012). Ab Mitte Januar 2012 begann dann eine mehrwöchige Frostperiode.

Bei den holzigen Anbausystemen konnte im Laufe des Jahres, ausgehend von hohen Anfangswerten im Winter 2011/2012, eine allgemeine Abnahme der elN-Gehalte festgestellt werden. Insbesondere mit dem einsetzenden Wachstum der Pappeln und Weiden im März/April 2012 nahmen die elNWerte kontinuierlich ab. Im April 2012 fielen die Temperaturen im Vergleich zum langjährigen Mittel höher aus (Abb. 5). Die geringsten Mengen konnten im AF nachgewiesen werden.

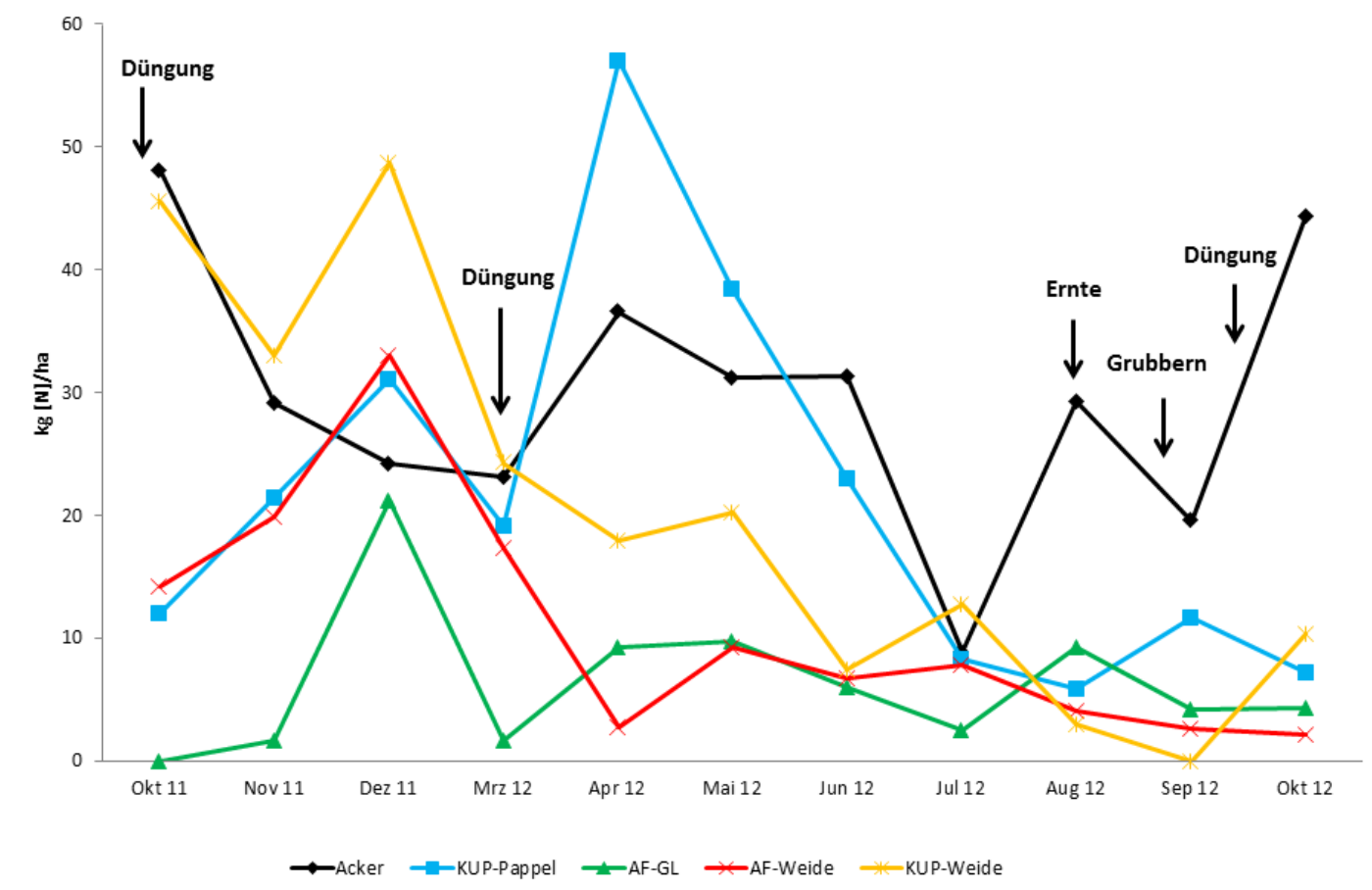

Abb. 11 Verlauf der mittleren monatlich extrahierbaren mineralischen Stickstoffmenge (eIN) für die Versuchsfläche Reiffenhausen von Oktober 2011 bis Oktober 2012 ( $n=3$ pro Teilfläche und Monat, im Oberboden 0-30 cm) 
Tabelle 12 Mittlere (Standardabweichung der monatlichen Mittelwerte) jährlich extrahierbare mineralische Stickstoffmenge (eIN, Oktober 2011 bis September 2012) im Oberboden (0-30 cm, n=36 pro Teilfläche)

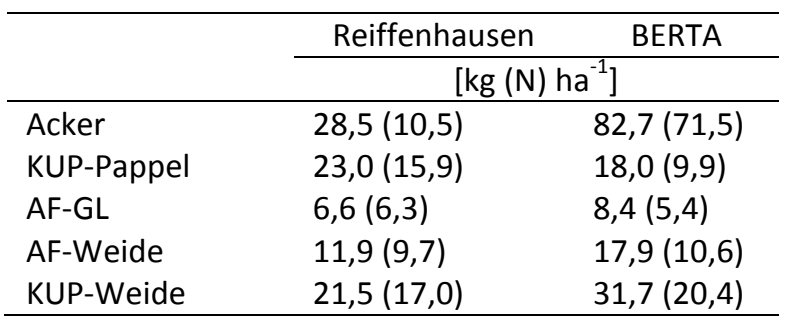

Durch das Ausbringen von stickstoffhaltigen Düngemitteln im März 2012 nahmen die elN-Werte im Ackerboden in Reiffenhausen zu. Bis zur Ernte des Winterrapses im August 2012 nahm die elNMenge durch das Pflanzenwachstum kontinuierlich ab.

Mit $32 \mathrm{~kg}(\mathrm{~N}) \mathrm{ha}^{-1}$ fiel die jährliche elN-Menge in BERTA im Flächenmittel deutlich höher aus als in Reiffenhausen. Auf allen Teilflächen dominiert mit über $91 \%$ Nitrat (elN-NO Ähnlich wie in Reiffenhausen konnten mit $83 \mathrm{~kg}(\mathrm{~N})$ ha $^{-1}$ die höchsten elN-Werte auf dem Acker beobachtet werden (Tabelle 12). Während der milden Wintermonate wurde elN freigesetzt, das jedoch aufgrund der fehlenden Ackerfrucht nicht genutzt wurde und somit eine Zunahme der eINWerte nach sich zog. Mit der einsetzenden Erwärmung und der Düngung von Ammonium und Nitrat im April nahmen die elN-Werte weiterhin zu. 2012 wurde bei einer Frühjahrsbeprobung der Thüringer Landesanstalt für Landwirtschaft eine mittlere elN-Menge von $57 \mathrm{~kg}(\mathrm{~N}) \mathrm{ha}^{-1}$ mit gleichmäßiger Verteilung auf den Ober- und Unterboden ermittelt (Thüringer Landesanstalt für Landwirtschaft 2012, Thüringer Landesanstalt für Landwirtschaft 2013). Auf der Versuchsfläche BERTA wurden bei der Beprobung am 09.03.2012 $28 \mathrm{~kg}(\mathrm{~N}) \mathrm{ha}^{-1}$ elN im Oberboden $(0-30 \mathrm{~cm}$ Bodentiefe) festgestellt.

Der im April ausgebrachte Mais führte erst im Juni 2012 zur Abnahme der elN-Werte (Abb. 12). Zwischen den Teilflächen in BERTA konnten keine signifikanten Unterschiede festgestellt werden. Die geringsten eIN-Mengen wurden auf den Anbausystemen AF und KUP-Pappel beobachtet (nicht signifikant). Mit Zunahme der Temperatur im Frühjahr 2012 und dem Wachstum der schnellwachsenden Gehölze nahmen im Sommer auch die elN-Werte langsam ab. Die elN-Mengen der KUP-Weide lagen prinzipiell über den Werten der KUP-Pappel (nicht signifikant). 


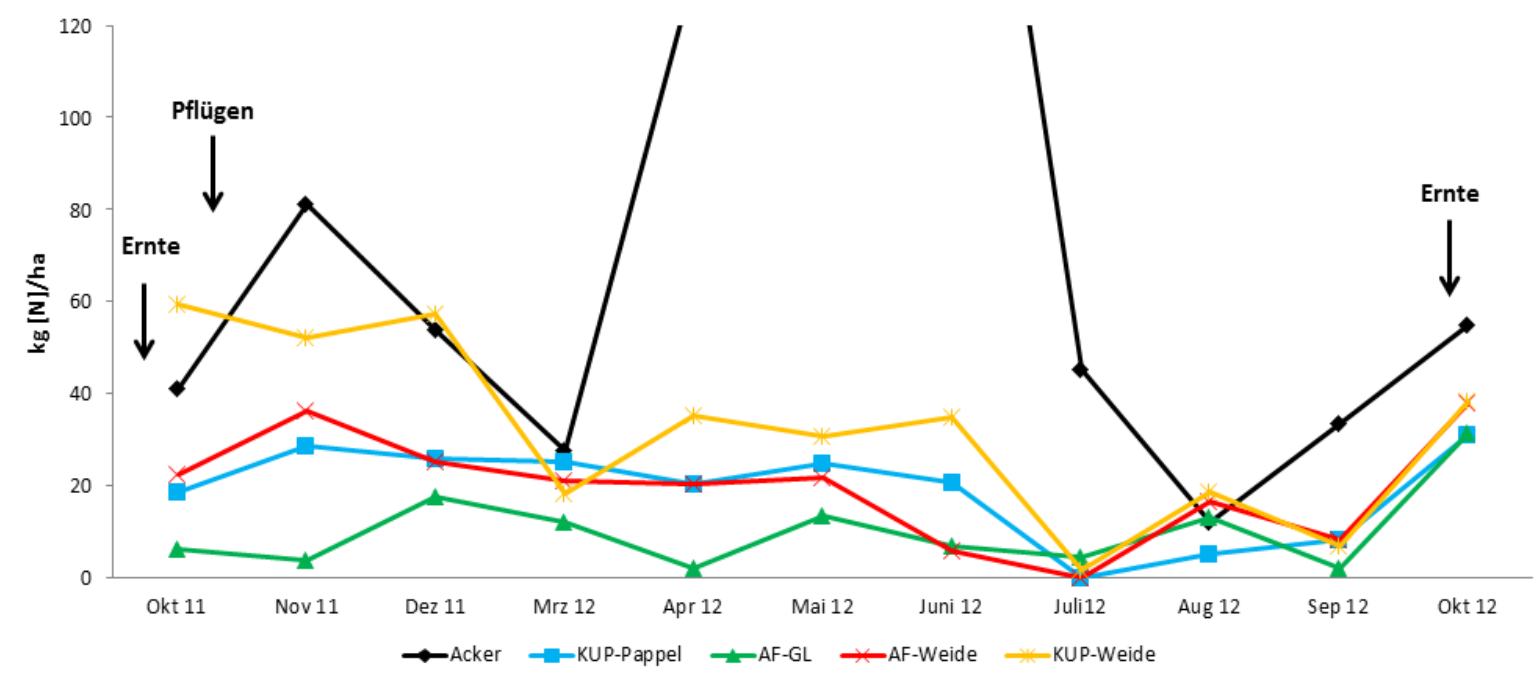

Abb. 12 Verlauf der mittleren monatlich extrahierbaren mineralischen Stickstoffmenge (eIN) für die Versuchsfläche BERTA von Oktober 2011 bis Oktober 2012 ( $n=3$ pro Teilfläche und Monat, im Oberboden 0-30 $\mathrm{cm})$

Zusammenfassend kann festgestellt werden, dass von der Teilfläche Acker in Reiffenhausen und BERTA über das gesamte Jahr hinweg die höchsten mittleren eIN-Mengen erreicht wurden. Dabei fielen die Mengen in BERTA höher aus als in Reiffenhausen. Die geringsten eIN-Werte wurden auf beiden Versuchsflächen auf der Teilfläche AF beobachtet, wobei der Unterschied zwischen der KUPPappel und AF-GL in Reiffenhausen signifikant ausfiel. Vergleicht man die KUP Teilflächen miteinander, können weder in Reiffenhausen noch für BERTA signifikante Unterschiede in den mittleren monatlichen eIN-Mengen festgestellt werden.

\subsubsection{Extrahierbarer organischer Stickstoff}

Im Flächenmittel wurden jährliche Gesamtmengen an extrahierbarem organischen Stickstoff (eON) von 19,5 kg (N) ha ${ }^{-1}$ für Reiffenhausen und 16,0 kg (N) ha ${ }^{-1}$ für BERTA ermittelt. Die höchsten Werte wurden auf der Teilfläche Acker mit rund $30 \mathrm{~kg}(\mathrm{~N}) \mathrm{ha}^{-1}$ in Reiffenhausen und ca. $20 \mathrm{~kg}(\mathrm{~N}) \mathrm{ha}^{-1}$ in BERTA beobachtet (Tabelle 13).

Tabelle 13 Mittlere (Standardabweichung der monatlichen Mittelwerte) extrahierbare organische Stickstoffmenge (Oktober 2011 bis September 2012) im Oberboden (0-30 cm, n=36 pro Teilfläche)

\begin{tabular}{|c|c|c|}
\hline & Reiffenhausen & BERTA \\
\hline & \multicolumn{2}{|c|}{$\left[\mathrm{kg}(\mathrm{N}) \mathrm{ha}^{-1}\right]$} \\
\hline Acker & $30,3(18,9)$ & $19,9(8,6)$ \\
\hline KUP-Pappel & $9,1(18,3)$ & $17,0(2,9)$ \\
\hline AF-GL & $27,8(9,8)$ & $15,7(3,7)$ \\
\hline AF-Weide & $6,3(6,4)$ & $15,2(4,4)$ \\
\hline KUP-Weide & $24,6(4,0)$ & $12,5(6,9)$ \\
\hline
\end{tabular}

In Reiffenhausen fielen die mittleren monatlichen eON-Werte der Teilfläche KUP-Weide im Vergleich zur KUP-Pappel und AF-Weide signifikant höher aus (Tabelle 13). Ferner konnten auf dem Acker im 
Vergleich zur KUP-Pappel und AF-Weide signifikant höhere mittlere monatliche eON-Mengen beobachtet werden. Die mittleren monatlichen Mengen an eON waren auf der Teilfläche AF-GL signifikant größer, als bei der KUP-Pappel bzw. AF-Weide. Die höchsten Werte wurden demzufolge auf den Teilflächen Acker, AF-GL und KUP-Weide nachgewiesen.

In der BERTA sind die mittleren monatlichen und jährlichen eON-Mengen der verschiedenen Teilflächen ähnlich (13 bis $20 \mathrm{~kg}(\mathrm{~N}) \mathrm{ha}^{-1}$ ) (Tabelle 13), es konnten keine signifikanten Unterschiede festgestellt werden.

Die höchsten Mengen an eON wurden in Reiffenhausen und BERTA auf der Teilfläche Acker beobachtet.

\subsubsection{Stickstoff-Nettomineralisation}

Infolge ansteigender Temperaturen nahm die N-Nettomineralisationsmenge ab Juli 2012 in Reiffenhausen stark zu (Abb. 13). In Reiffenhausen wurde im Mittel auf der Teilfläche KUP-Weide mit ca. $28 \mathrm{~kg}(\mathrm{~N}) \mathrm{ha}^{-1}$ von März bis Oktober 2012 die größte Menge an elN-NH $\mathrm{N}_{4}$ und elN-NO $\mathrm{N}_{3}$ produziert. Die geringste Menge an elN konnte mit $13 \mathrm{~kg}(\mathrm{~N}) \mathrm{ha}^{-1}$ für die Teilfläche Acker beobachtet werden (Tabelle 14). Als Stickstoffform dominierte auf allen Teilflächen bei der N-Nettomineralisation mit mehr als $90 \%$ elN-NO Teilflächen konnten keine signifikanten Unterschiede in den monatlichen $\mathrm{N}$ Nettomineralisationsmengen festgestellt werden.

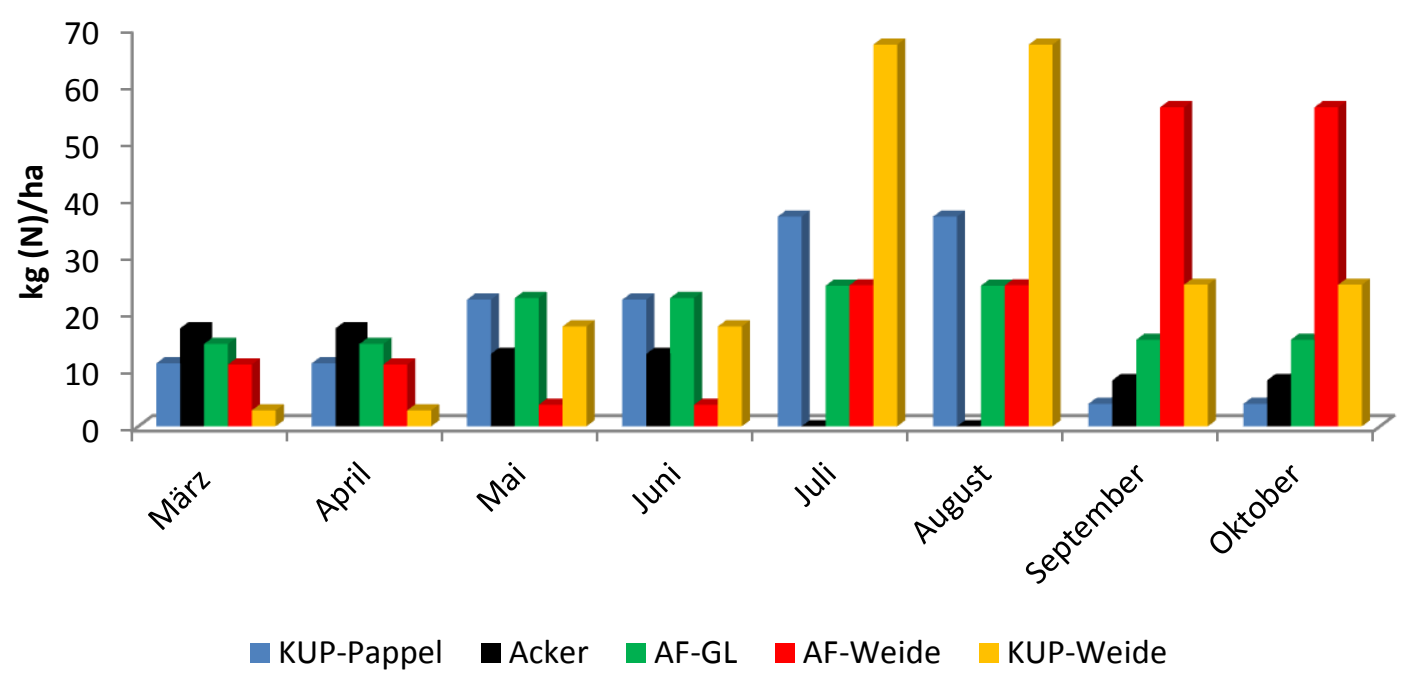

Abb. 13 Verlauf der mittleren monatlichen N-Nettomineralisationsmengen im Zeitraum März bis Oktober 2012 für die Versuchsfläche Reiffenhausen ( $n=4$ pro Monat und Teilfläche) 
Tabelle 14 Mittlere (Standardabweichung der monatlichen Mittelwerte) N-Nettomineralisationsmenge in kg (N) ha ${ }^{-1}$ für den Zeitraum März bis Oktober 2012 für die Versuchsflächen Reiffenhausen und BERTA ( $n=32$ pro Teilfläche)

\begin{tabular}{lccccc}
\hline & KUP-Pappel & AF-GL & AF-Weide & KUP-Weide & Acker \\
\hline Reiffenhausen & $18,6(13,3)$ & $19,3(4,8)$ & $24,0(21,4)$ & $28,1(25,5)$ & $12,7(4,1)$ \\
BERTA & $9,0(4,9)$ & $19,2(32,3)$ & $13,7(22,1)$ & $11,8(12,3)$ & $-21,7(59,8)$ \\
\hline
\end{tabular}

Auch in BERTA konnten zwischen den einzelnen Teilflächen keine signifikanten Unterschiede in den mittleren monatlichen N-Nettomineralisationsmengen festgestellt werden. Auffällig sind jedoch die Monate Mai, Juni, Juli und August 2012 auf der Teilfläche Acker (Abb. 14). Die ermittelten negativen $\mathrm{N}$-Nettomineralisationsmengen lassen sich als Immobilisierung mineralischer Stickstofffraktionen deuten (Schimming et al. 1995). Der Anteil an eIN-NO ${ }_{3}$ an der N-Nettomineralisation betrug auf allen Teilflächen in BERTA mindestens $97 \%$.

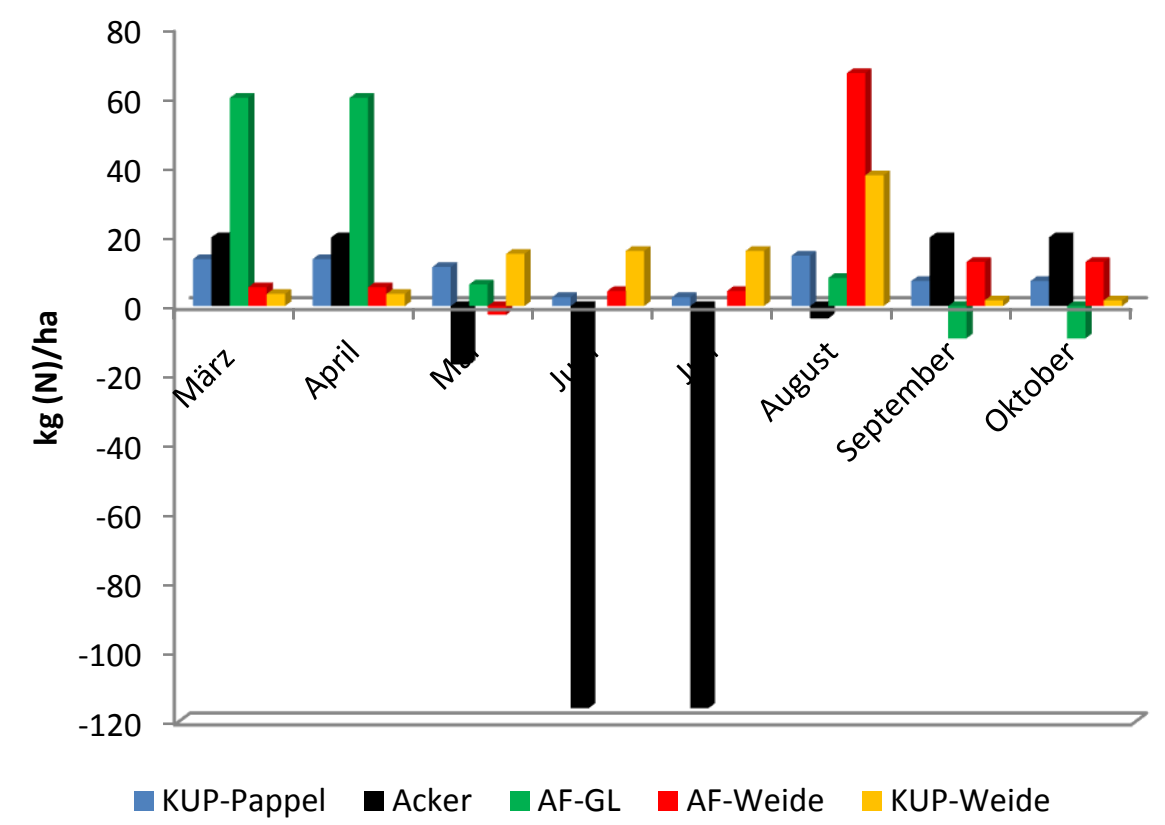

Abb. 14 Verlauf der mittleren monatlichen N-Nettomineralisationsmengen im Zeitraum März bis Oktober 2012 für die Versuchsfläche BERTA ( $n=4$ pro Monat und Teilfläche)

Zwischen den verschiedenen Teilflächen sowohl in Reiffenhausen als auch in BERTA konnten keine signifikanten Unterschiede in der mittleren monatlichen $\mathrm{N}$-Nettomineralisationsmenge beobachtet werden.

\subsubsection{Holzige Biomasse}

Die Anwuchsrate der Pappeln und Weiden in KUP und AF nach einjähriger Standdauer sind in Tabelle 15 dargestellt. Die größten Anwuchserfolge wurden in Reiffenhausen und BERTA mit ca. $90 \%$ bei Weide beobachtet. Pappeln erreichten mit ca. $60 \%$ in Reiffenhausen und ca. $70 \%$ in BERTA 
wesentlich geringere Anwuchserfolge (Hartmann et al. 2014, Hartmann u. Lamersdorf 2014a, Hartmann u. Lamersdorf 2014b).

Tabelle 15 Anzahl der vitalen und nachgesteckten Bäume pro ha und Anwuchsrate in Reiffenhausen und BERTA auf den verschiedenen Teilflächen (KUP-Pappel, KUP-Weide, AF-Weide, Pflanzung im Frühjahr 2011, Vollaufnahme Frühjahr 2012)

\begin{tabular}{lcccccccc}
\hline & \multicolumn{3}{c}{ Reiffenhausen } & & \multicolumn{3}{c}{ BERTA } \\
\cline { 2 - 4 } \cline { 6 - 8 } & KUP-Pappel & KUP-Weide & AF-Weide & & KUP-Pappel & KUP-Weide & AF-Weide \\
\hline vitale Bäume [ha ${ }^{-1}$ ] & 5.589 & 10.449 & 11.206 & & 6.613 & 10.772 & 10.858 \\
nachgesteckte Bäume $\left[\mathrm{ha}^{-1}\right.$ ] & 3.301 & 1.401 & 644 & & 2.277 & 1.078 & 992 \\
Anwuchsrate [\%] & 62,9 & 88,2 & 94,6 & & 74,4 & 90,9 & 91,6 \\
\hline
\end{tabular}

In Reiffenhausen wies die Pappel im Vergleich zur Weide signifikant geringere Höhen und Brusthöhendurchmesser auf (Tabelle 16). Dies kann damit begründet werden, dass die vielen nachgesteckten Bäume, die zufällig mit erfasst wurden, nur geringe Höhen und Brusthöhendurchmesser hatten. Die Erträge zwischen KUP-Pappel und KUP-Weide fielen hingegen ähnlich aus. Aufgrund der geringeren Baumanzahl pro ha wurden im AF geringere Erträge an holziger Biomasse als bei KUP beobachtet (Tabelle 16). Unter Berücksichtigung der gleichen Baumanzahl wie bei der KUP-Weide wurden Zuwächse von ca. 0,9 $t_{\text {atro }} h^{-1} a^{-1}$ ermittelt (Tabelle 16) (Hartmann u. Lamersdorf 2014a, Hartmann u. Lamersdorf 2014b).

Die geringste Höhe für BERTA wurde beim holzigen Anbausystem AF-Weide ermittelt. Der BHD war signifikant am größten bei der KUP-Pappel, wobei hier auch die höchsten Zuwächse mit ca. 1,6 $t_{\text {atro }}$ ha $^{-1} \mathrm{a}^{-1}$ beobachtet wurden (Hartmann u. Lamersdorf 2014a, Hartmann u. Lamersdorf 2014b).

Tabelle 16 Mittlere Höhe (Standardabweichung), Brusthöhendurchmesser (BHD, Standardabweichung), Ertrag und Zuwachs der Pappeln und Weiden in Reiffenhausen und BERTA auf den Teilflächen (KUP-Pappel, KUPWeide, AF-Weide, $n=240$ pro Teilfläche Reiffenhausen, $n=$ mind. 160 pro Teilfläche BERTA) nach zweijähriger Standdauer

\begin{tabular}{|c|c|c|c|c|}
\hline & & KUP- Pappel & KUP-Weide & AF-Weide \\
\hline \multirow[t]{4}{*}{ Reiffenhausen } & Höhe [m] & $2,2^{*}+(1,3)$ & $2,6 *(1,1)$ & $2,6+(0,7)$ \\
\hline & $\mathrm{BHD}[\mathrm{mm}]$ & $6,4^{*+}(6.6)$ & $10,7^{*}(8,2)$ & $10,3+(6,7)$ \\
\hline & Zuwachs $\left[\mathrm{t}_{\text {atro }}\right.$ ha $\left.\mathrm{a}^{-1}\right]$ & 1,00 & 1,09 & $0,42^{\circ}(0,93)^{\circ}$ \\
\hline & Ertrag $\left[\mathrm{t}_{\text {atro }} \mathrm{ha}^{-1}\right]$ & 2,00 & 2,17 & $0,84^{\circ}(1,87)^{\circ}$ \\
\hline \multirow[t]{4}{*}{ BERTA } & Höhe [m] & $2,4^{\dagger \wedge}(1,1)$ & $1,8 *+(0,7)$ & $1,6^{* \wedge}(0,7)$ \\
\hline & $\mathrm{BHD}[\mathrm{mm}]$ & $8,8^{\dagger \wedge}(7,5)$ & $4,5+(5,6)$ & $4,5^{\wedge}(5,4)$ \\
\hline & Zuwachs $\left[\mathrm{t}_{\text {tatro }}\right.$ ha $\left.\mathrm{a}^{-1}\right]$ & 1,59 & 0,59 & n.b. \\
\hline & Ertrag $\left[\mathrm{t}_{\text {atro }} \mathrm{ha}^{-1}\right]$ & 3,18 & 1,18 & n.b. \\
\hline
\end{tabular}

Gleiche Symbole bedeuten signifikante Unterschiede $(p \leq 0,05)$ zwischen den Teilflächen für die Höhe und BHD

- tatsächliche Baumanzahl von 5.330 ha $^{-1}$ für die Teilfläche Agroforst-Weide (AF-Weide), hinzukommen noch die Erträge vom Grünland mit ca. $8 \mathrm{t}$ Trockenmasse ha ${ }^{-1} \mathrm{a}^{-1}$ (Ehret et al. 2013)

•kalkulierte Baumanzahl von $11.850 \mathrm{ha}^{-1}$ als reine Kurzumtriebsplantage (KUP) mit Weide n.b. nicht bestimmt

Zur Beurteilung des Ernährungszustandes und des Nährstoffbedarfs von schnellwachsenden Gehölzen dienen Blattanalysen (Bungart u. Hüttl 2004, Elowson u. Rytter 1988, Röhricht u. Ruscher 2009, Rytter u. Ericsson 1993). Dabei wurden Grenzwerte für eine optimale und ausreichende 
Ernährung ermittelt, die es ermöglichen Entscheidungen im Hinblick auf notwendige Düngungsmaßnahmen zu treffen (Röhricht u. Ruscher 2009).

In Tabelle 17 ist der Ernährungszustand der Bestände nach zweijähriger Standdauer dargestellt. Es wird ersichtlich, dass die Pappel stets optimal mit allen Nährstoffen (außer Kalium in BERTA und Magnesium in Reiffenhausen) versorgt ist. Bei der Weide können bei allen Nährelementen stets ausreichende Nährstoffgehalte beobachtet werden.

Tabelle 17 Mittlere Nährstoffgehalte in Blättern (Standardabweichung) der Pappeln und Weiden in KUP und AF in Reiffenhausen (Reiff.) und BERTA für 2012 ( $n=$ mindestens 5 pro Teilfläche, Optimum nach Röhricht u. Ruscher 2009, Ausreichend nach Jug et al. 1999a)

\begin{tabular}{lllllll}
\hline & & $\mathrm{N}$ & $\mathrm{P}$ & $\mathrm{K}$ & $\mathrm{Ca}$ & $\mathrm{Mg}$ \\
\cline { 3 - 7 } & & \multicolumn{5}{c}{$\left[\mathrm{mg} \mathrm{g}^{-1}\right]$} \\
\hline KUP-Weide & Reiff. & $24,2(3,9)$ & $3,8(0,6)$ & $14,4(2,1)$ & $14,8(2,9)$ & $3,5(0,8)$ \\
& BERTA & $26,5(1,9)$ & $2,4(0,2)$ & $12,6(2,0)$ & $11,7(0,4)$ & $3,7(0,5)$ \\
& Optimum & $26-32$ & $3,5-5$ & $16-18$ & $11-14$ & $1,8-2,3$ \\
& Ausreichend & 22 & 1,8 & 8 & 3,5 & 1,6 \\
AF-Weide & & & & & & \\
& Reiff. & $25,6(1,4)$ & $3,9(0,4)$ & $16,2(0,5)$ & $11,7(2,0)$ & $2,4(0,3)$ \\
& BERTA & $22,0(3,6)$ & $2,3(0,2)$ & $11,2(0,6)$ & $13,8(1,1)$ & $3,4(0,1)$ \\
& Optimum & $26-32$ & $3,5-5$ & $16-18$ & $11-14$ & $1,8-2,3$ \\
& Ausreichend & 22 & 1,8 & 8 & 3,5 & 1,6 \\
& & & & & & \\
KUP-Pappel & Reiff. & $21,6(1,2)$ & $3,9(0,5)$ & $18,5(0,4)$ & $5,9(0,2)$ & $1,4(0,1)$ \\
& BERTA & $21,6(1,8)$ & $2,7(0,4)$ & $10,8(1,0)$ & $11,8(1,0)$ & $2,2(0,3)$ \\
& Optimum & $18-25$ & $1,8-3$ & $12-18$ & $3-15$ & $2-3$ \\
& Ausreichend & 16 & 1 & 7 & 5,4 & 1,3 \\
\hline
\end{tabular}

In Reiffenhausen und BERTA wurde der höchste Ertrag, unter Berücksichtigung des Anwuchserfolges, bei der KUP-Pappel ermittelt. Der Anwuchserfolg fiel bei der Weide in KUP und AF stets höher aus, im Vergleich zur Pappel in KUP.

Basierend auf den jährlichen Zuwachsdaten nach 2-jähriger Standdauer und den Nährstoffgehalten aus 2012 ergeben sich je nach holzigem Anbausystem 2,7 bis 10,7 $\mathrm{kg}(\mathrm{N}) \mathrm{ha}^{-1} \mathrm{a}^{-1}$ an $\mathrm{N}$-Entzug durch die holzige Biomasse (Tabelle 18). Bezieht man die Stickstoffabfuhr auf einen einheitlichen Zuwachs von $1 t_{\text {atro }} h^{-1} a^{-1}$, werden von der KUP-Pappel im Vergleich zur KUP-Weide und AF-Weide sowohl in Reiffenhausen als auch in BERTA höhere N-Mengen entzogen. 
Tabelle 18 Jährlicher Zuwachs an holziger Biomasse, mittlere (Standardabweichung, SD) Nährstoffvorräte im Boden (austauschbare Kationen und $P_{t}, 0-30 \mathrm{~cm}$ Bodentiefe), mittlere (SD) elN-Menge $(0-30 \mathrm{~cm})$ sowie mittlere (SD) jährliche Nährstofffestlegung in der holzigen Biomasse für die verschiedenen Teilflächen (KUP-Pappel, KUP-Weide, AF-Weide) in Reiffenhausen (Reiff.) und BERTA

\begin{tabular}{|c|c|c|c|c|c|c|c|c|}
\hline & & $\begin{array}{c}\text { Zuwachs } \\
{\left[\mathrm{kg}_{\text {atro }}\right.} \\
\left.\mathrm{ha}^{-1} \mathrm{a}^{-1}\right]\end{array}$ & & $\mathrm{Ca}$ & K & $\mathrm{Mg}$ & $P_{t}$ & $\begin{array}{l}\text { N (Biomasse) /elN*/elN } \\
(\text { Vegetationsperiode)^ }\end{array}$ \\
\hline \multirow[t]{4}{*}{ Reiff. } & $\begin{array}{c}\text { KUP- } \\
\text { Pappel }\end{array}$ & 1.000 & $\begin{array}{c}\text { Festlegung } \\
\text { holzige }\end{array}$ & $\begin{array}{c}5,3 \\
(1,0)\end{array}$ & $\begin{array}{c}3,7 \\
(0,6)\end{array}$ & $\begin{array}{c}0,5 \\
(0,1)\end{array}$ & $\begin{array}{c}1,3 \\
(0,2)\end{array}$ & $7,4(0,9)$ \\
\hline & $\begin{array}{l}\text { KUP- } \\
\text { Weide }\end{array}$ & 1.090 & $\begin{array}{l}\text { Biomasse } \\
{\left[\mathrm{kg} \mathrm{ha}^{-1} \mathrm{a}^{-1}\right]}\end{array}$ & $\begin{array}{c}3,4 \\
(0,5)\end{array}$ & $\begin{array}{c}2,7 \\
(0,4)\end{array}$ & $\begin{array}{c}0,6 \\
(0,1)\end{array}$ & $\begin{array}{c}1,0 \\
(0,1)\end{array}$ & $5,5(0,9)$ \\
\hline & $\begin{array}{c}\text { AF- } \\
\text { Weide }\end{array}$ & $420 / 930^{\circ}$ & & $\begin{array}{c}1,6 \\
(0,2) / \\
3,6 \\
(0,5)\end{array}$ & $\begin{array}{c}1,1 \\
(0,1) / \\
2,4 \\
(0,2)\end{array}$ & $\begin{array}{c}0,2 \\
(0,0) / \\
0,4 \\
(0,0)\end{array}$ & $\begin{array}{c}0,4 \\
(0,0) / \\
1,0 \\
(0,1)\end{array}$ & $2,7(0,3) / 6,0(0,7)$ \\
\hline & & & $\begin{array}{l}\text { Vorrat Boden } \\
{\left[\mathrm{kg} \mathrm{ha}^{-1}\right]}\end{array}$ & 13.795 & 1.504 & 983 & 2.680 & $188^{*} / 188^{\wedge}$ \\
\hline \multirow[t]{3}{*}{ BERTA } & $\begin{array}{c}\text { KUP- } \\
\text { Pappel }\end{array}$ & 1.590 & $\begin{array}{l}\text { Festlegung } \\
\text { holzige }\end{array}$ & $\begin{array}{c}16,2 \\
(13,4)\end{array}$ & $\begin{array}{c}5,2 \\
(2,0)\end{array}$ & $\begin{array}{c}0,9 \\
(0,3)\end{array}$ & $\begin{array}{c}1,4 \\
(0,4)\end{array}$ & $10,7(1,6)$ \\
\hline & $\begin{array}{l}\text { KUP- } \\
\text { Weide }\end{array}$ & 590 & $\begin{array}{l}\text { Biomasse } \\
{\left[\mathrm{kg} \mathrm{ha}^{-1} \mathrm{a}^{-1}\right]}\end{array}$ & $\begin{array}{c}2,5 \\
(0,3)\end{array}$ & $\begin{array}{c}1,6 \\
(0,2)\end{array}$ & $\begin{array}{c}0,4 \\
(0,0)\end{array}$ & $\begin{array}{c}0,4 \\
(0,0)\end{array}$ & $3,6(0,4)$ \\
\hline & & & $\begin{array}{l}\text { Vorrat Boden } \\
{\left[\mathrm{kg} \mathrm{ha}^{-1}\right]}\end{array}$ & 23.589 & 587 & 2.161 & 2.074 & $225^{*} / 92^{\wedge}$ \\
\hline
\end{tabular}

*eIN: mittlere extrahierbare Menge an mineralischem Stickstoff im Boden für den Zeitraum Oktober 2011 bis September 2012, $\mathrm{n}=3$ pro Monat und Teilfläche

$\wedge$ eIN (Vegetationsperiode): mittlere N-Nettomineralisationsmenge im Boden in der Vegetationsperiode (März bis Oktober 2012), $\mathrm{n}=4$ pro Monat und Teilfläche

${ }^{\circ}$ kalkulierte Baumanzahl von 11.850 ha $^{-1}$ als reine Kurzumtriebsplantage (KUP) mit Weide

Die im Boden vorhandenen elN-Vorräte und das durch die N-Nettomineralisation in der Vegetationsperiode bereitgestellte eIN liegen für beide Versuchsflächen im Oberboden $(0-30 \mathrm{~cm})$ um ein Vielfaches über der mittleren jährlichen Festlegung in der holzigen Biomasse (elN pro Jahr: mindestens 25-fach in Reiffenhausen und 21-fach in BERTA, eIN in Vegetationsperiode durch NNettomineralisation mindestens 25-fach in Reiffenhausen und 8-fach in BERTA). Ähnlich verhält es sich auch für die anderen Nährelemente, sodass davon ausgegangen werden kann, dass über mehrere Ernteperioden der Nährstoffbedarf ohne zusätzliche Düngung gesichert ist.

Bezieht man die jährliche Nährstoffabfuhr auf einen einheitlichen Zuwachs von $1 t_{\text {atro }} \mathrm{ha}^{-1} \mathrm{a}^{-1}$, benötigt in Reiffenhausen und BERTA die Pappel in KUP für ihr Wachstum mehr Calcium, Kalium, Phosphor und Stickstoff als die Weide in KUP und AF. Der jährliche Gesamtentzug an Calcium, Kalium, Phosphor und Stickstoff durch Ernte der holzigen Biomasse nach zweijähriger Standdauer ist bei KUP-Pappel stets am höchsten. 


\subsubsection{Laubproduktion und Umsatz}

Der Eintrag von Laub ist für die Versorgung der Pflanzen mit Nährstoffen bedeutsam (Polyakova u. Billor 2007, Pérez-Corona et al. 2006). Durch die Zersetzung des Laubes wird dem Boden ein Teil der Nährstoffe, die zuvor von den Pflanzen aufgenommen wurden, zurückgeführt (Guo u. Sims 1999, Prescott 2005).

Die mittleren Nährstoffgehalte des eingetragenen Laubes in den Jahren 2011 und 2012 sind in Tabelle 19 aufgeführt. In 2012 wurden in Reiffenhausen keine signifikanten Unterschiede in den Nund Ca-Gehalten zwischen dem Laub der Weiden und Pappeln beobachtet. Signifikante Unterschiede konnten hingegen bei den P-, K- und Mg-Gehalten festgestellt werden. In 2012 wurden zwischen dem Pappel- und Weidenlaub in BERTA bei allen Nährstoffen signifikante Unterschiede festgestellt.

Tabelle 19 Mittlere (Standardabweichung) Nährstoffgehalte und C/N- bzw. C/P-Verhältnis des eingetragenen Laubes im Herbst 2011 und 2012 für die Versuchsflächen Reiffenhausen und BERTA ( $n=6$ pro Jahr und Teilfläche)

\begin{tabular}{|c|c|c|c|c|c|c|c|c|c|}
\hline & & & $\mathrm{N}$ & $\mathrm{P}$ & $\mathrm{K}$ & $\mathrm{Ca}$ & $\mathrm{Mg}$ & \multirow[t]{2}{*}{$\mathrm{C} / \mathrm{N}$} & \multirow[t]{2}{*}{$\mathrm{C} / \mathrm{P}$} \\
\hline & & & \multicolumn{5}{|c|}{$\left[\mathrm{mg} \mathrm{g}^{-1}\right]$} & & \\
\hline \multirow[t]{11}{*}{ Reiffenhausen } & 2011 & KUP- & $8,5^{* \dagger}$ & 1,2 & $14,9 *+$ & $19,8 * \dagger$ & $1,1^{*}$ & $55,2^{*}+$ & 589,0 \\
\hline & & Pappel & $(1,2)$ & $(1,0)$ & $(0,6)$ & $(1,2)$ & $(0,2)$ & $(7,4)$ & $(307,8)$ \\
\hline & & AF- & $11,5^{*}$ & 1,0 & $11,0^{*}$ & $23,4^{*}$ & $1,4+$ & $42,7^{*}$ & 482,4 \\
\hline & & Weide & $(1,9)$ & $(0,3)$ & $(2,0)$ & $(2,2)$ & $(0,1)$ & $(6,9)$ & $(107,4)$ \\
\hline & & KUP- & $13,1+$ & 0,8 & $9,5+$ & $24,3+$ & $4,7 *+$ & $36,4+$ & 572,4 \\
\hline & & Weide & $(1,4)$ & $(0,1)$ & $(2,1)$ & $(2,6)$ & $(0,4)$ & $(3,9)$ & $(44,7)$ \\
\hline & 2012 & KUP- & 11,8 & $3,1^{*}$ & $10,1^{*}$ & 23,8 & $0,9 * \dagger$ & 41,1 & $151,9 *$ \\
\hline & & Pappel & $(3,4)$ & $(0,6)$ & $(2,9)$ & $(3,3)$ & $(0,1)$ & $(9,6)$ & $(27,9)$ \\
\hline & & AF- & 10,2 & $3,2+$ & 7,7 & 24,9 & $1,4+\wedge$ & 46,3 & $150,4+$ \\
\hline & & Weide & $(0,4)$ & $(0,5)$ & $(1,9)$ & $(2,9)$ & $(0,2)$ & $(2,5)$ & $(25,7)$ \\
\hline & & $\begin{array}{l}\text { KUP- } \\
\text { Weide }\end{array}$ & $9,6(0,7)$ & $\begin{array}{l}1,8^{* \dagger} \\
(0,2)\end{array}$ & $\begin{array}{l}7,1^{*} \\
(2,0)\end{array}$ & $\begin{array}{l}24,3 \\
(1,0)\end{array}$ & $\begin{array}{l}3,7 * \wedge \\
(0,3)\end{array}$ & $\begin{array}{l}49,1 \\
(3,6)\end{array}$ & $\begin{array}{l}258,0 * \dagger \\
(30,0)\end{array}$ \\
\hline \multirow[t]{12}{*}{ BERTA } & 2011 & KUP- & $13,0 *$ & 0,8 & 3,7 & $35,7 *+$ & $3,6^{*}$ & 34,4 & 574,1 \\
\hline & & Pappel & $(1,6)$ & $(0,2)$ & $(1,2)$ & $(2,0)$ & $(0,3)$ & $(4,3)$ & $(112,3)$ \\
\hline & & AF- & $12,7+$ & $0,6^{*}$ & 4,8 & $16,9^{*}$ & 4,0 & 34,2 & 668,6 \\
\hline & & Weide & $(2,2)$ & $(0,1)$ & $(1,4)$ & $(1,9)$ & $(0,4)$ & $(8,4)$ & $(60,3)$ \\
\hline & & KUP- & $16,6 *+$ & $0,7^{*}$ & 4,1 & $19,4 \dagger$ & $4,3^{*}$ & 28,3 & 626,8 \\
\hline & & Weide & $(2,4)$ & $(0,1)$ & $(0,6)$ & $(3,1)$ & $(0,5)$ & $(4,1)$ & $(46,0)$ \\
\hline & 2012 & KUP- & $6,0 *+$ & $0,8^{*}$ & $4,9 *$ & $35,3 *+$ & $3,3 *+$ & $74,9 *+$ & $663,0 *$ \\
\hline & & Pappel & $(0,6)$ & $(0,4)$ & $(1,1)$ & $(2,7)$ & $(0,4)$ & $(6,8)$ & $(259,7)$ \\
\hline & & $A F-$ & $9,3 * \wedge$ & $1,6^{*}+$ & $3,9+$ & $30,9^{*}$ & $4,3 * \wedge$ & $51,0 * \wedge$ & $300,8 *+$ \\
\hline & & Weide & $(2,4)$ & $(0,5)$ & $(1,5)$ & $(3,2)$ & $(0,6)$ & $(10,0)$ & $(91,0)$ \\
\hline & & KUP- & $11,9+\wedge$ & $1,1^{\dagger}$ & $6,6 *+$ & $30,2+$ & $5,3+\wedge$ & $40,1 \dagger^{\wedge}$ & $458,8+$ \\
\hline & & Weide & $(1,2)$ & $(0,2)$ & $(1,4)$ & $(3,3)$ & $(0,7)$ & $(4,8)$ & $(75,4)$ \\
\hline
\end{tabular}

Gleiche Symbole bedeuten signifikante Unterschiede $(p \leq 0,05)$ zwischen den Teilflächen für die verschiedenen Nährstoffe und Nährstoffverhältnisse

Die eingetragene Laubmenge lag 2012 in Reiffenhausen zwischen $137 \mathrm{~kg} \mathrm{ha}^{-1}$ und $942 \mathrm{~kg} \mathrm{ha}^{-1}$ (Tabelle 20). Für BERTA konnten im gleichen Zeitraum Laubeinträge von $35 \mathrm{~kg} \mathrm{ha}^{-1}$ bis $1.394 \mathrm{~kg} \mathrm{ha}^{-1}$ beobachtet werden. Die höchsten Laubeinträge wurden stets bei der Pappel festgestellt, sodass absolut gesehen mit dem Pappellaub auch die größten Nährstoffmengen dem Boden entzogen und später zurückgeführt werden (außer Mg für KUP-Weide in Reiffenhausen, 2012). Mit zunehmendem 
Alter der Bäume (2011 zu 2012) erhöhte sich die eingetragene Laubmenge, wobei die prozentuale Zunahme bei der Pappel in KUP im Vergleich zur Weide in KUP und AF höher ausfiel.

Tabelle 20 Mittlere (Standardabweichung) jährlich eingetragene Nährstoffmenge mit dem Laub ( $\mathrm{n}=6$ pro Jahr und Teilfläche) in Reiffenhausen und BERTA

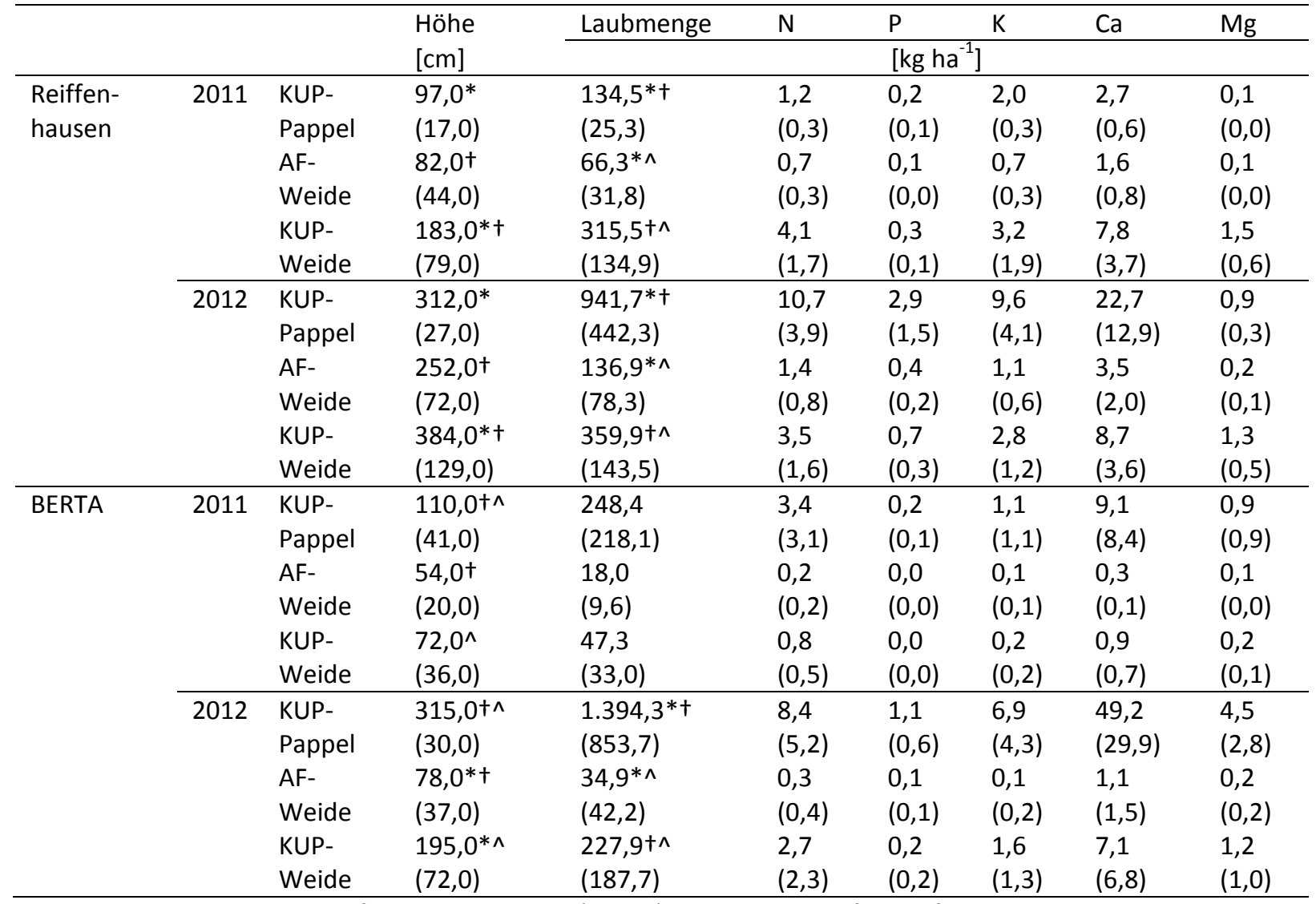

Gleiche Symbole bedeuten signifikante Unterschiede $(p \leq 0,05)$ zwischen den Teilflächen für die Höhe und Laubmenge, sign. Unterschiede zwischen den Teilflächen wurden für die Nährstoffe nicht bestimmt

Die mittleren Nährstoffgehalte des ausgebrachten Laubes in den Litterbags sind in der Tabelle 21 zusammengefasst. Das Weidenlaub wies zu Beginn der Ausbringung signifikant höhere N-, Ca- und Mg-Gehalte als das Pappellaub auf. 
Tabelle 21 Mittlere (Standardabweichung) Nährstoffgehalte des ausgebrachten Laubes in Litterbags zu Beginn und am Ende des Versuchs nach einjähriger Ausbringungszeit ( $n=4$ pro Teilfläche und Zeitpunkt: Beginn bzw. Ende) in Reiffenhausen (Reiff.) und BERTA

\begin{tabular}{|c|c|c|c|c|c|c|c|c|c|}
\hline & & & $\mathrm{N}$ & $P$ & $\mathrm{~K}$ & $\mathrm{Ca}$ & $\mathrm{Mg}$ & \multirow[t]{2}{*}{$\mathrm{C} / \mathrm{N}$} & \multirow[t]{2}{*}{$C / P$} \\
\hline & & & \multicolumn{5}{|c|}{$\left[\mathrm{mg} \mathrm{g}^{-1}\right]$} & & \\
\hline & Beginn & Weide & $15,5^{*}$ & 1,1 & 9,6 & $44,6 *$ & $4,8^{*}$ & 28,9 & 425,5 \\
\hline & & & $(0,8)$ & $(0,1)$ & $(1,7)$ & $(3,1)$ & $(0,3)$ & $(1,9)$ & $(30,2)$ \\
\hline & & Pappel & $12,6^{*}$ & 1,3 & 12,0 & $37,4^{*}$ & $2,8^{*}$ & 35,3 & 362,1 \\
\hline & & & $(2,2)$ & $(0,3)$ & $(1,3)$ & $(1,0)$ & $(0,3)$ & $(6,2)$ & $(100,2)$ \\
\hline \multirow[t]{6}{*}{ Reiff. } & Ende & KUP- & 23,6 & 1,8 & 2,1 & 43,3 & 2,0 & 17,7 & 230,8 \\
\hline & & Pappel & $(3,5)$ & $(0,2)$ & $(0,2)$ & $(5,4)$ & $(0,4)$ & $(2,2)$ & $(23,7)$ \\
\hline & & AF- & 20,9 & 1,4 & 2,3 & 48,5 & 2,4 & 21,5 & 312,6 \\
\hline & & Weide & $(1,0)$ & $(0,1)$ & $(0,8)$ & $(1,9)$ & $(0,3)$ & $(1,5)$ & $(37,0)$ \\
\hline & & KUP- & 18,3 & 1,2 & 6,8 & 35,3 & 5,9 & 19,9 & 318,3 \\
\hline & & Weide & $(0,7)$ & $(0,1)$ & $(2,8)$ & $(6,5)$ & $(1,7)$ & $(1,8)$ & $(59,7)$ \\
\hline \multirow{6}{*}{ BERTA } & Ende & KUP- & 16,7 & 1,2 & 3,6 & 35,4 & 3,0 & 20,5 & 279,5 \\
\hline & & Pappel & $(1,2)$ & $(0,1)$ & $(0,7)$ & $(3,6)$ & $(0,3)$ & $(2,3)$ & $(30,2)$ \\
\hline & & $A F-$ & 18,2 & 1,0 & 4,4 & 38,0 & 4,9 & 20,7 & 368,3 \\
\hline & & Weide & $(0,8)$ & $(0,1)$ & $(0,7)$ & $(1,9)$ & $(0,5)$ & $(1,3)$ & $(49,0)$ \\
\hline & & KUP- & 17,8 & 1,2 & 5,1 & 38,0 & 6,9 & 19,8 & 305,5 \\
\hline & & Weide & $(0,9)$ & $(0,0)$ & $(0,9)$ & $(3,7)$ & $(0,9)$ & $(0,7)$ & $(24,0)$ \\
\hline
\end{tabular}

Gleiche Symbole bedeuten signifikante Unterschiede $(p \leq 0,05)$ zwischen dem Weiden- und Pappellaub für die verschiedenen Nährstoffe, diese wurden nur zu Beginn des Versuchs ermittelt

Nach einjähriger Ausbringungszeit der Litterbags wurde der mit 67 \% größte Gewichtsverlust beim Pappellaub in KUP in Reiffenhausen beobachtet (Abb. 15). Vom Laub der Weide in KUP und AF blieben nach einem Jahr ca. 50 \% des Ursprungsgewichts übrig.

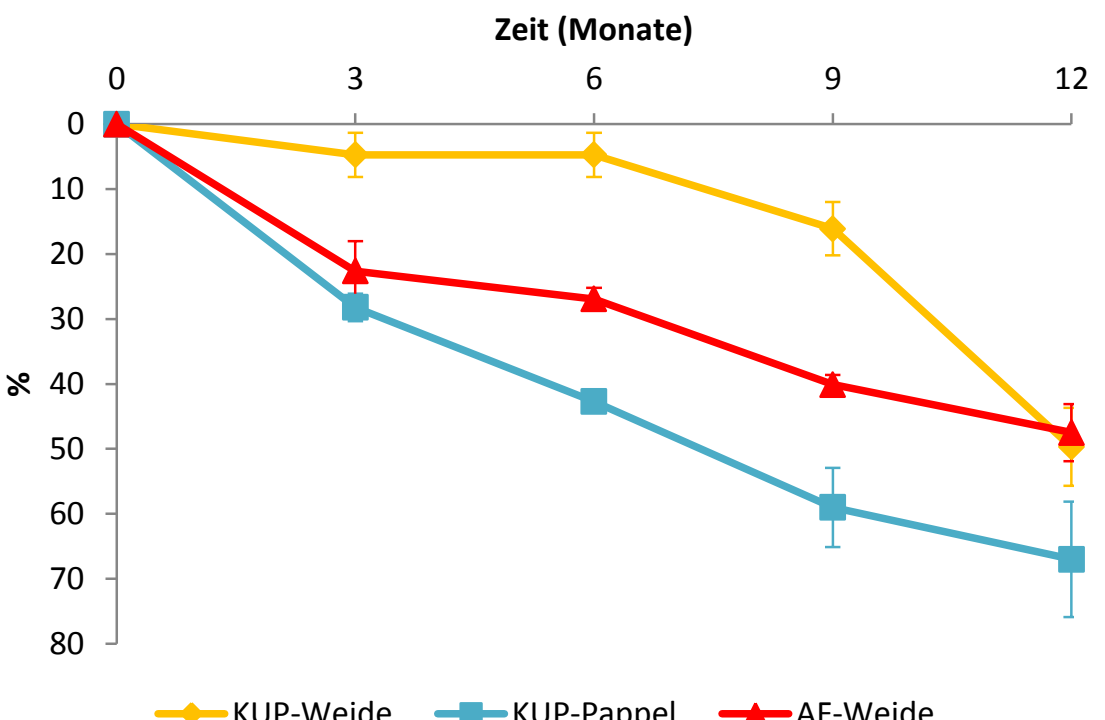

Abb. 15 Mittlerer (Standardabweichung) Trockengewichtsverlust des Pappel- und Weidenlaubes in den ausgebrachten Litterbags über einen Zeitraum von 12 Monaten in Reiffenhausen ( $n=4$ pro Entnahmetermin, insgesamt 4 Entnahmetermine im vierteljährlichen Turnus: nach 3, 6, 9 und 12 Monaten)

In BERTA wurde nach einjähriger Ausbringungszeit der größte Gewichtsverlust beim Laub der Pappel und Weide in KUP mit ca. $55 \%$ der Litterbags ermittelt (Abb. 16). Am geringsten war der Gewichtsverlust beim Weidenlaub im AF mit $45 \%$ pro Jahr. 


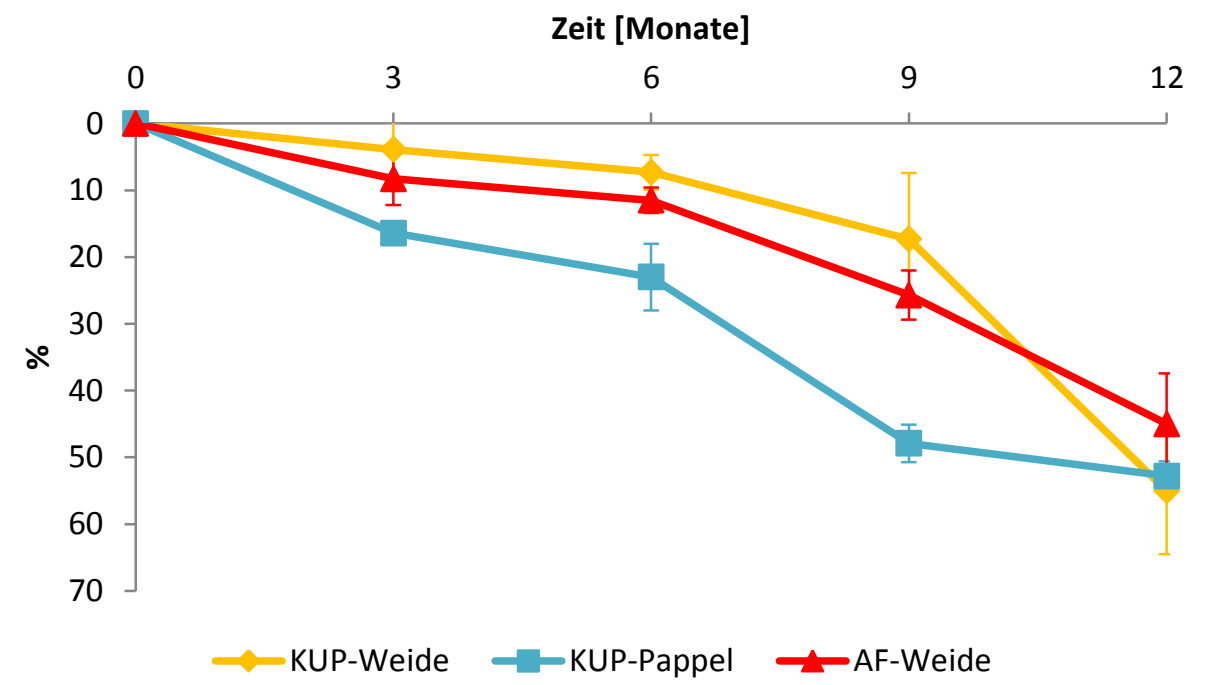

Abb. 16 Mittlerer (Standardabweichung) Trockengewichtsverlust des Pappel- und Weidenlaubes in den ausgebrachten Litterbags über einen Zeitraum von 12 Monaten in BERTA ( $n=4$ pro Entnahmetermin, insgesamt 4 Entnahmetermine im vierteljährlichen Turnus: nach 3, 6, 9 und 12 Monaten)

In Reiffenhausen und BERTA wurde das Pappellaub in den Litterbags schneller zersetzt als das Weidenlaub; in Reiffenhausen fiel dieser Unterschied signifikant aus (Tabelle 22).

Tabelle 22 Mittlere tägliche $\left(\mathrm{k} \mathrm{d}^{-1}\right)$ und jährliche Zersetzungsrate $\left(\mathrm{k} \mathrm{a}^{-1}\right)$ des Weiden- und Pappellaubes in KUP und AF ( $n=4$ pro Teilfläche) in Reiffenhausen und BERTA

\begin{tabular}{llllllll}
\hline & \multicolumn{3}{c}{ Reiffenhausen } & & \multicolumn{3}{c}{ BERTA } \\
\cline { 2 - 4 } \cline { 7 - 8 } & KUP-Weide & KUP-Pappel & AF-Weide & & KUP-Weide & KUP-Pappel & AF-Weide \\
\hline $\mathrm{k} \mathrm{d}^{-1}$ & $0,00298^{*}$ & $0,00434^{*}+$ & $0,00296 \dagger$ & & 0,00335 & 0,00366 & 0,00289 \\
$\mathrm{SD}$ & 0,00039 & 0,00059 & 0,00021 & & 0,00082 & 0,0012 & 0,00028 \\
$\mathrm{k} \mathrm{a}^{-1}$ & 1,08934 & 1,58319 & 1,07994 & & 1,22093 & 1,33462 & 1,05458 \\
$\mathrm{R}^{2}$ & 0,87 & 0,96 & 0,94 & & 0,87 & 0,97 & 0,96 \\
\hline
\end{tabular}

Gleiche Symbole bedeuten signifikante Unterschiede $(p \leq 0,05)$ zwischen den Teilflächen für die tägliche und jährliche Zersetzungsrate

Nach 12 Monaten wurden von den anfänglichen Stickstoffmengen $29-40$ \% in Reiffenhausen und 36$48 \%$ in BERTA freigesetzt (Tabelle 23). Zwischen den holzigen Anbausystemen sowohl in Reiffenhausen als auch in BERTA konnten keine signifikanten Unterschiede in der $\mathrm{N}-, \mathrm{P}_{-}, \mathrm{K}$ und $\mathrm{Mg}-$ Freisetzung beobachtet werden. Zwischen den verschiedenen Teilflächen in Reiffenhausen konnten signifikante Unterschiede in der Freisetzung von Calcium beobachtet werden. Kalium wurde von allen Nährelementen am stärksten freigesetzt, gefolgt von Calcium. 
Tabelle 23 Mittlerer (Standardabweichung) Trockengewichtsverlust und mittlere (Standardabweichung) Nährstofffreisetzung in \% nach 12 Monaten für Pappel- und Weidenlaub in KUP und AF ( $n=4$ pro Teilfläche) in Reiffenhausen und BERTA

\begin{tabular}{llllllll}
\hline & \multicolumn{3}{c}{ Reiffenhausen } & & \multicolumn{3}{c}{ BERTA } \\
\cline { 2 - 5 } \cline { 6 - 8 } & KUP-Weide & KUP-Pappel & AF-Weide & & KUP-Weide & KUP-Pappel & AF-Weide \\
\hline Gewicht & $49,7^{*}(6,0)$ & $67,0^{*}+(8,9)$ & $47,5 \dagger(4,4)$ & & $55,0(9,5)$ & $52,8(2,2)$ & $45,0(7,6)$ \\
Stickstoff & $40,3(9,1)$ & $37,4(19,3)$ & $29,4(4,4)$ & & $47,9(12,9)$ & $36,0(14,8)$ & $35,6(5,8)$ \\
Phosphor & $44,0(11,7)$ & $48,5(26,6)$ & $27,6(13,7)$ & & $50,2(13,0)$ & $52,9(8,3)$ & $46,4(4,0)$ \\
Kalium & $62,3(18,5)$ & $94,3(1,6)$ & $87,1(5,2)$ & & $75,1(8,9)$ & $85,6(2,8)$ & $74,6(3,3)$ \\
Calcium & $60,1^{*}(9,5)$ & $62,5+(6,8)$ & $42,6^{*}+(6,9)$ & & $61,3(10,6)$ & $55,4(1,7)$ & $52,7(8,9)$ \\
Magnesium & $36,8(22,8)$ & $75,6(10,2)$ & $73,3(4,3)$ & & $34,7(15,5)$ & $48,8(8,5)$ & $43,6(8,3)$ \\
\hline
\end{tabular}

Gleiche Symbole bedeuten signifikante Unterschiede $(p \leq 0,05)$ zwischen den verschiedenen Teilflächen für das Gewicht und die verschiedenen Nährstoffe

Das meiste Laub wurde in Reiffenhausen und BERTA von der KUP-Pappel produziert. Innerhalb eines Jahres wird dieses im Vergleich zum Weidenlaub schneller zersetzt, wobei Nährstoffe freigesetzt werden, die erneut aufgenommen werden können oder sich im Bodenwasser wiederfinden. Die Nährstofffreisetzung fiel beim Pappel- und Weidenlaub gleich groß aus (ausgenommen Calcium). Absolut gesehen gelangen mit dem Pappellaub aber mehr Nährstoffe in den Boden zurück, da die Laubmenge bedeutend größer ausfiel.

\subsubsection{Boden- \& Sickerwasser}

Von Dezember 2011 bis November 2012 wurden mit ca. 27 mg (N) L L-1 auf der Teilfläche KUP-Weide in Reiffenhausen die höchsten $\mathrm{DNO}_{3}$-Konzentrationen im Bodenwasser beobachtet (Abb. 17). Auf der KUP-Pappel und AF-Weide fielen die Konzentrationen mit 2,7 und 2,9 $\mathrm{mg}(\mathrm{N}) \mathrm{L}^{-1}$ relativ ähnlich aus.

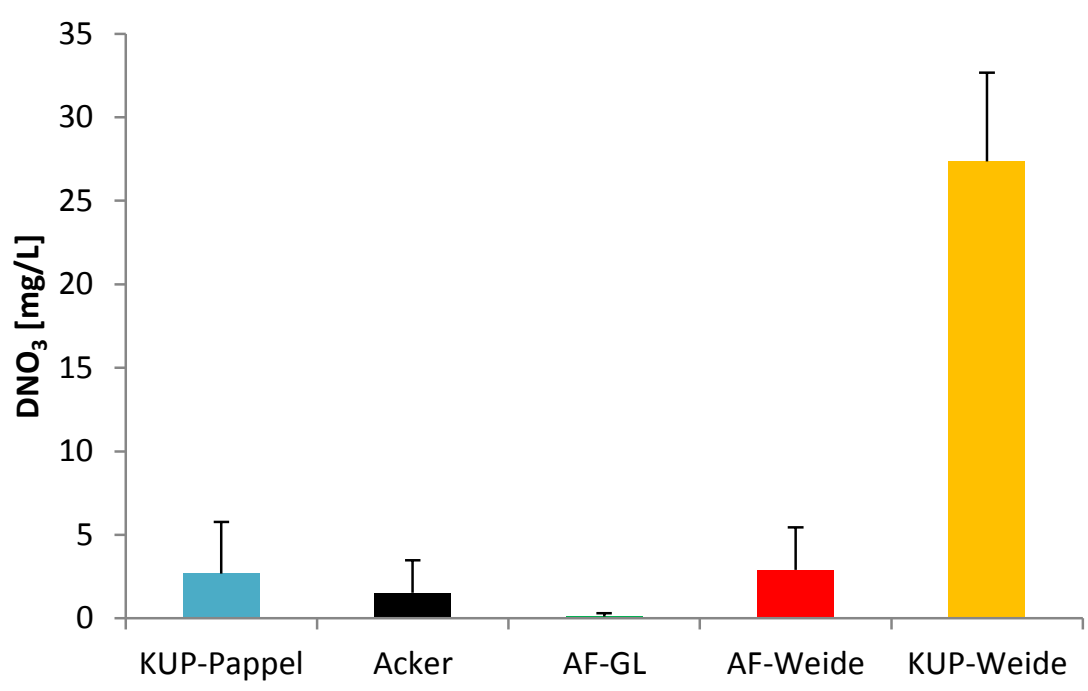

Abb. 17 Mittlere (positive Standardabweichung) jährliche Nitratkonzentration im Bodenwasser in $60 \mathrm{~cm}$ Bodentiefe in Reiffenhausen (Entnahmezeitpunkte an denen Bodenlösung gewonnen wurde: KUP-Pappel: $n=6$, Acker: $n=8$, AF-GL: $n=6, A F-W e i d e: ~ n=4, K U P-W e i d e: ~ n=4)$ für den Zeitraum Dezember 2011 bis November 2012 
Im Allgemeinen nehmen die $\mathrm{DNO}_{3}$-Konzentrationen in der Vegetationszeit in Reiffenhausen ab, was mit dem N-Konsum durch die Pappeln und Weiden erklärt werden kann (Anhang 1). Die geringsten gemessenen $\mathrm{DNO}_{3}$-Konzentrationen wurden unter der Nutzung $\mathrm{AF}-\mathrm{GL}$ mit maximal 0,3 $\mathrm{mg}(\mathrm{N}) \mathrm{L}^{-1}$ ermittelt.

Für den Zeitraum Dezember 2011 bis November 2012 ist der Verlauf der geschätzten Nitratausträge mit dem Sickerwasser ab $60 \mathrm{~cm}$ Bodentiefe in Reiffenhausen in der Abb. 18 dargestellt. Der geringste Nitrataustrag wurde mit insgesamt $2,5 \mathrm{~kg}(\mathrm{~N}) \mathrm{ha}^{-1} \mathrm{a}^{-1}$ für die Teilfläche KUP-Pappel ermittelt. Auf der Teilfläche KUP-Weide wurden jährliche Gesamt-Nitratausträge von 22,3 kg (N) ha-1 $\mathrm{a}^{-1}$ bzw. 4,4 kg (N) $\mathrm{ha}^{-1} \mathrm{a}^{-1}$ für die Teilfläche AF-Weide bestimmt. Insbesondere der Zeitraum Dezember 2011 bis März 2012 ist durch erhöhte Austräge gekennzeichnet.

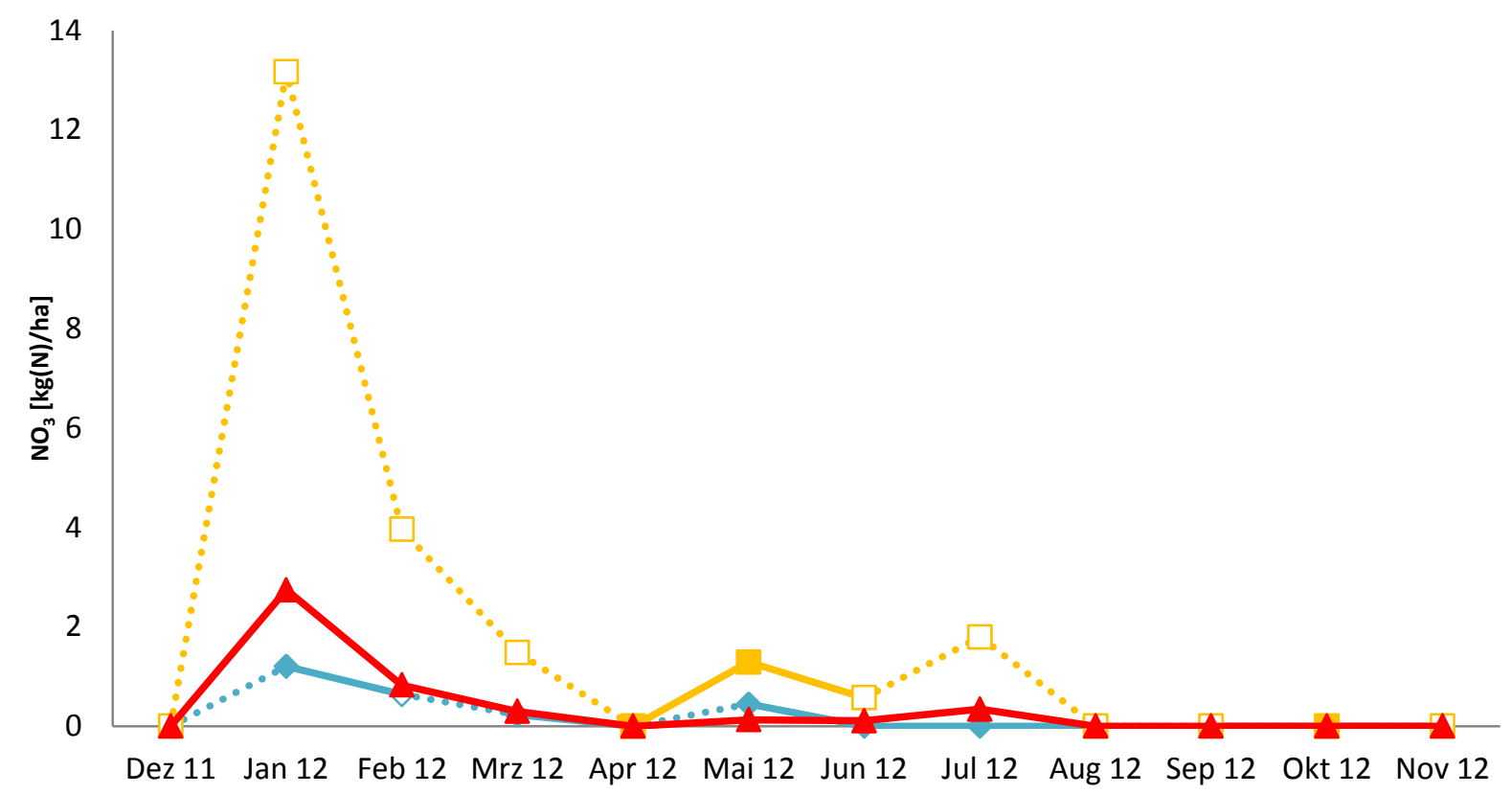

Abb. 18 Mittlerer monatlicher Nitrataustrag für die Teilflächen KUP-Pappel (blau), KUP-Weide (orange) und AFWeide (rot) in $60 \mathrm{~cm}$ Bodentiefe für die Versuchsfläche Reiffenhausen, die durchgezogenen Linien und gefüllten Symbole sind tatsächlich gemessene Werte $\left(\mathrm{DNO}_{3}\right.$-Konzentrationen im Bodenwasser), gestrichelte Linien und ungefüllte Symbole bedeuten interpolierte Werte ( $\mathrm{DNO}_{3}$-Konzentrationen im Bodenwasser)

In der "Thüringer Ackerebene" konnten im Mittel für das Jahr 2012 die höchsten $\mathrm{DNO}_{3}$ Konzentrationen im Bodenwasser auf den Teilflächen KUP-Weide und Acker beobachtet werden (Abb. 19). Besonders gering fiel die mittlere $\mathrm{DNO}_{3}$-Konzentration im $\mathrm{AF}$ ( $\mathrm{AF}-\mathrm{GL}+\mathrm{AF}-\mathrm{W}$ ) aus. 


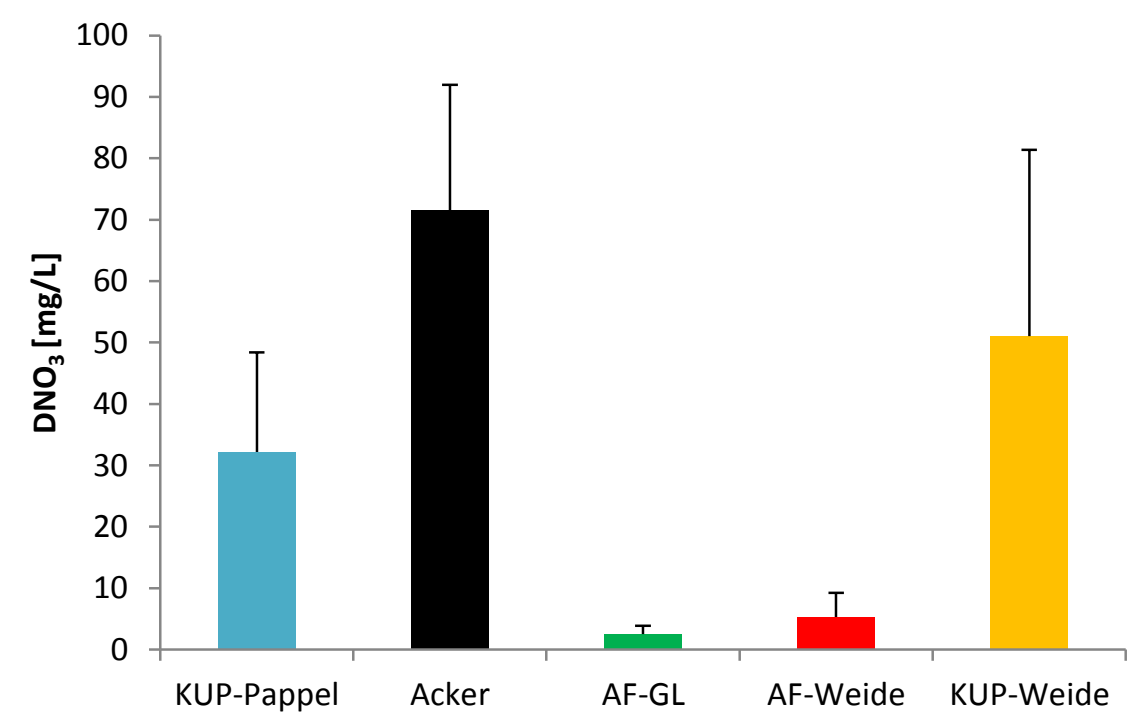

Abb. 19 Mittlere (positive Standardabweichung) jährliche Nitratkonzentration im Bodenwasser in $60 \mathrm{~cm}$ Bodentiefe in BERTA (Entnahmezeitpunkte an denen Bodenlösung gewonnen wurde: KUP-Pappel: $n=5$, Acker: n=6, AF-GL: $n=5$, AF-Weide: $n=4$, KUP-Weide: $n=6$ ) für den Zeitraum Januar 2012 bis Dezember 2012

Der Verlauf der Nitratausträge mit dem Sickerwasser ab $60 \mathrm{~cm}$ Bodentiefe für den Zeitraum Januar 2012 bis Dezember 2012 in BERTA ist in der Abbildung 20 dargestellt. In den Monaten März 2012 bis November 2012 wurde kein Sickerwasser gebildet, sodass kein Nitrataustrag stattfand. In den Monaten Januar und Februar 2012 wurde der geringste Austrag an Nitrat mit 3,9 kg (N) ha ${ }^{-1}$ für die Teilfläche AF-Weide ermittelt. Auf den Teilflächen KUP-Weide bzw. KUP-Pappel wurden im gleichen Zeitraum Nitratausträge von $35,3 \mathrm{~kg}(\mathrm{~N}) \mathrm{ha}^{-1} \mathrm{bzw} .11,7 \mathrm{~kg}(\mathrm{~N}) \mathrm{ha}^{-1}$ bestimmt.

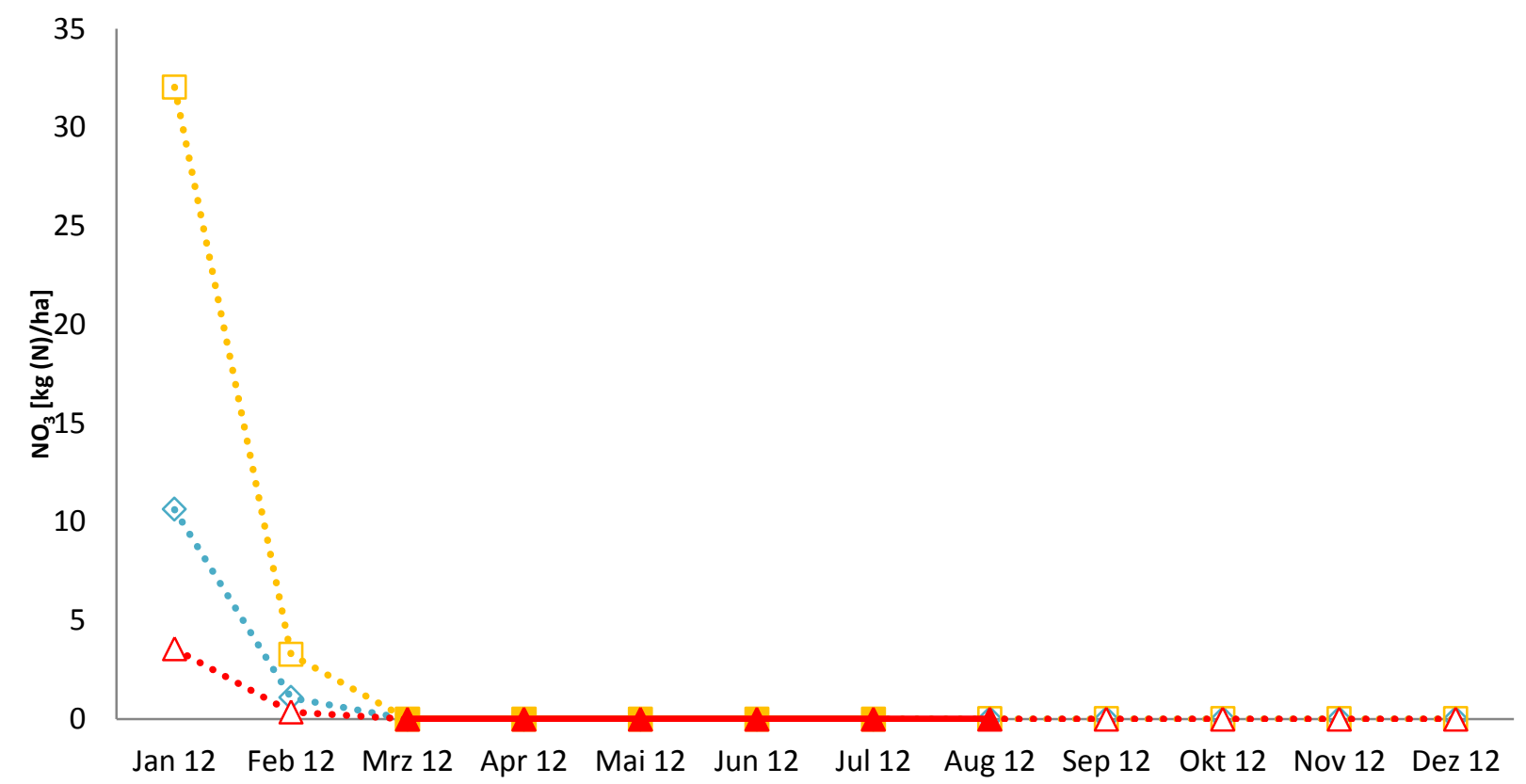

Abb. 20 Mittlerer monatlicher Nitrataustrag für die Teilflächen KUP-Pappel (blau), KUP-Weide (orange) und AFWeide (rot) in $60 \mathrm{~cm}$ Bodentiefe für die Versuchsfläche BERTA, die durchgezogenen Linien und gefüllten Symbole sind tatsächlich gemessene Werte $\left(\mathrm{DNO}_{3}\right.$-Konzentrationen im Bodenwasser), gestrichelte Linien und ungefüllte Symbole bedeuten interpolierte Werte $\left(\mathrm{DNO}_{3}\right.$-Konzentrationen im Bodenwasser) 
Die höchsten Konzentrationen an gelöstem Nitrat im Bodenwasser in $60 \mathrm{~cm}$ Tiefe wurden unabhängig von der Versuchsfläche stets bei der Teilfläche KUP-Weide beobachtet (Anhang 1, Anhang 2). Demzufolge fielen auch die Nitratausträge beim holzigen Anbausystem KUP-Weide am höchsten aus.

\subsubsection{Deposition}

In die Atmosphäre wird Stickstoff hauptsächlich in Form von Stickoxiden $\left(\mathrm{N}_{2} \mathrm{O}\right.$, NO und $\left.\mathrm{NO}_{2}\right)$ und Ammoniak $\left(\mathrm{NH}_{3}\right)$ bzw. Ammonium $\left(\mathrm{NH}_{4}\right)$ eingetragen (Neumann 2008). $\mathrm{NO}$ und $\mathrm{NO}_{2}$ entstehen vor allem in Kraft- und Heizwerken, bei der Industriefeuerung, beim Hausbrand und im Straßenverkehr (Fier et al. 2013). $\mathrm{N}_{2} \mathrm{O}$ entsteht als Beiprodukt in der chemischen Industrie oder im Boden (Neumann 2008). Ammonium bzw. Ammoniak stammt insbesondere aus der Landwirtschaft, durch intensive Tierhaltung und die damit verbundene Ausbringung wirtschaftseigener Dünger (Gülle, Jauche, Stallmist) sowie bei der Verwendung von stickstoffhaltigem Mineraldünger (Fier et al. 2013, Neumann 2008). Im Niederschlag finden sich als Folgeprodukte hauptsächlich Nitrat und Ammonium. In Reiffenhausen wurde eine Jahresstickstoffdeposition von rund $11,0 \mathrm{~kg}(\mathrm{~N}) \mathrm{ha}^{-1} \mathrm{a}^{-1}$ beobachtet. Dabei beläuft sich der Anteil an $\mathrm{DNH}_{4}$ auf 5,4 kg (N) ha $\mathrm{a}^{-1}$ und an $\mathrm{DNO}_{3}$ auf 4,8 kg (N) ha $\mathrm{ha}^{-1}$.

In BERTA wurde im gleichen Zeitraum mehr $\mathrm{DNH}_{4}\left(7,8 \mathrm{~kg}(\mathrm{~N}) \mathrm{ha}^{-1} \mathrm{a}^{-1}\right)$ als $\mathrm{DNO}_{3}\left(5,0 \mathrm{~kg}(\mathrm{~N}) \mathrm{ha}^{-1} \mathrm{a}^{-1}\right)$ deponiert. Insgesamt wurde ein Gesamtstickstoffeintrag von 13,4 kg (N) ha $\mathrm{h}^{-1} \mathrm{a}^{-1}$ beobachtet.

Die jährliche atmosphärische N-Deposition, gemessen mit Bulk-Sammlern, fiel mit $11,0 \mathrm{~kg}(\mathrm{~N}) \mathrm{ha}^{-1} \mathrm{a}^{-1}$ (Reiffenhausen) bzw. 13,4 kg (N) ha ${ }^{-1} a^{-1}$ (BERTA) zwischen den Versuchsflächen recht ähnlich aus.

\subsubsection{N-Bilanz und N-Nutzungseffzienz}

In Tabelle 24 werden die jährlichen Stickstoffein- und -austräge für die holzigen Anbausysteme KUPPappel, KUP-Weide und AF-Weide in Reiffenhausen und BERTA dargestellt. Als Eintrag für die NBilanz werden die N-Nettomineralisation und N-Deposition berücksichtigt (Kapitel 3.2.3 \& Kapitel 3.2.7). Trotz hoher Standardabweichungen wurde tendenziell auf beiden Standorten durch Mineralisation mehr Stickstoff unter KUP-Weide und AF-Weide als unter KUP-Pappel produziert. Die jährlichen N-Depositionsmengen fallen auf beiden Versuchsflächen hingegen ähnlich aus.

Für die N-Austräge wurden die geschätzten Lachgasemissionen und N-Sickerwasserausträge berücksichtigt (Kapitel 3.2.6). Hinzu kommt noch der Stickstoff, der dem System mit der Ernte der holzigen Biomasse entzogen wird. Der N-Entzug mit der holzigen Biomasse ist sowohl in Reiffenhausen als auch in BERTA bei der KUP-Pappel am größten. Der N-Austrag mit dem Sickerwasser fällt für beide Versuchsflächen am höchsten unter KUP-Weide aus. Die Lachgasausträge betragen für Reiffenhausen maximal $2 \mathrm{~kg}(\mathrm{~N}) \mathrm{ha} \mathrm{a}^{-1} \mathrm{a}^{-1}$ und für BERTA maximal $1 \mathrm{~kg}(\mathrm{~N}) \mathrm{ha}^{-1} \mathrm{a}^{-1}$. 
Der größte N-Gesamtaustrag ( $=\sum$ Austrag, Tabelle 24$)$ wurde auf beiden Versuchsflächen für das holzige Anbausystem KUP-Weide ermittelt. Dies liegt in den hohen N-Austrägen mit dem Sickerwasser begründet. Während in Reiffenhausen der geringste N-Austrag bei der KUP-Pappel festgestellt wurde, wurde in BERTA der geringste N-Austrag beim holzigen Anbausystem AF-Weide beobachtet.

Unter Berücksichtigung der gesamten N-Eintrags- und -Austragsflüsse wurden in der Etablierungsphase der holzigen Anbausysteme in Reiffenhausen und BERTA N-Bilanzen von -14,7 kg (N) ha $a^{-1} a^{-1}$ bis 22,5 kg (N) ha-1 $a^{-1}$ ermittelt (Tabelle 24). Während sich in Reiffenhausen für alle holzigen Anbausysteme positive N-Bilanzen ergaben, konnte auf der Versuchsfläche BERTA eine negative N-Bilanz für die KUP-Pappel und KUP-Weide festgestellt werden. Positive Bilanzen bedeuten, dass trotz $\mathrm{N}$-Austrägen mit Sickerwasser, Lachgas und Ernte der holzigen Biomasse Stickstoff im System zurückgehalten wird.

Tabelle 24 Mittlerer (Standardabweichung) jährlicher Ein- und Austrag (Flüsse) an Stickstoff (N) und Stickstoffbilanz (N-Bilanz) für die verschiedenen holzigen Anbausysteme (KUP-Pappel, KUP-Weide, AF-Weide) in Reiffenhausen (Reiff.) und BERTA

\begin{tabular}{|c|c|c|c|c|c|c|}
\hline & \multicolumn{2}{|c|}{ KUP-Pappel } & \multicolumn{2}{|c|}{ KUP-Weide } & \multicolumn{2}{|c|}{ AF-Weide } \\
\hline & Reiff. & BERTA & Reiff. & BERTA & Reiff. & BERTA \\
\hline $\begin{array}{l}\text { N-Eintrag: N-Nettomineralisation }{ }^{\wedge}\left[\mathrm{kg}(\mathrm{N}) \mathrm{ha}^{-}\right. \\
\left.{ }^{1} \mathrm{a}^{-1}\right]\end{array}$ & $\begin{array}{l}18,6 \\
(13,3)\end{array}$ & $\begin{array}{l}9,0 \\
(4,9)\end{array}$ & $\begin{array}{l}28,1 \\
(25,5)\end{array}$ & $\begin{array}{l}11,8 \\
(12,3)\end{array}$ & $\begin{array}{l}23,9 \\
(21,4)\end{array}$ & $\begin{array}{l}13,7 \\
(22,0)\end{array}$ \\
\hline N-Eintrag: N-Deposition ${ }^{\circ}\left[\mathrm{kg}(\mathrm{N}) \mathrm{ha}^{-1} \mathrm{a}^{-1}\right]$ & $\begin{array}{l}11,0 \\
(0,4)\end{array}$ & $\begin{array}{l}13,4 \\
(0,6)\end{array}$ & $\begin{array}{l}11,0 \\
(0,4)\end{array}$ & $\begin{array}{l}13,4 \\
(0,6)\end{array}$ & $\begin{array}{l}11,0 \\
(0,4)\end{array}$ & $\begin{array}{l}13,4 \\
(0,6)\end{array}$ \\
\hline$\sum$ Eintrag & 29,6 & 22,4 & 39,1 & 25,2 & 34,9 & 27,1 \\
\hline $\begin{array}{l}\text { N-Austrag: } \\
\text { Holzige Biomasse }^{\dagger}\left[\mathrm{kg}(\mathrm{N}) \mathrm{ha}^{-1} \mathrm{a}^{-1}\right]\end{array}$ & $\begin{array}{l}7,4 \\
(0,9)\end{array}$ & $\begin{array}{l}10,7 \\
(1,6)\end{array}$ & $\begin{array}{l}5,5 \\
(0,9)\end{array}$ & $\begin{array}{l}3,6 \\
(0,4)\end{array}$ & $\begin{array}{l}6,0 \\
(0,7)\end{array}$ & $\begin{array}{l}3,6 \\
(0,4) *\end{array}$ \\
\hline $\begin{array}{l}\text { N-Austrag: } \\
\text { Sickerwasser }^{\bullet}\left[\mathrm{kg}(\mathrm{N}) \mathrm{ha}^{-1} \mathrm{a}^{-1}\right]\end{array}$ & 2,5 & 11,7 & 22,3 & 35,3 & 4,4 & 3,9 \\
\hline $\begin{array}{l}\text { N-Austrag: } \\
\text { Lachgasemission }^{\bullet}\left[\mathrm{kg}(\mathrm{N}) \mathrm{ha} \mathrm{a}^{-1} \mathrm{a}^{-1}\right]\end{array}$ & $\leq 2$ & $\leq 1$ & $\leq 2$ & $\leq 1$ & $\leq 2$ & $\leq 1$ \\
\hline$\sum$ Austrag & 11,9 & 23,4 & 29,8 & 39,9 & 12,4 & 8,5 \\
\hline N-Bilanz ( $\Sigma$ Eintrag - $\Sigma$ Austrag) & 17,7 & $-1,0$ & 9,3 & $-14,7$ & 22,5 & 18,6 \\
\hline $\begin{array}{l}\text { ^Vegetationsperiode } 2012 \text { mit Buried Bags } \\
\text { Beprobungstermin } \\
{ }^{\circ} \text { Bulk Deposition im 2-Wochen Turnus beprok } \\
{ }^{\dagger} \text { allometrische Biomassefunktion mit } 15 \text { Prok } \\
\text { und Rinde) erfasst } \\
\text { 'geschätzte Werte: Nitratmessungen in Bode } \\
12 \text { bis Dez. } 12 \text { in BERTA, } 4 \text { Saugkerzen pro } \\
\text { KUP-Pappel modelliert, dieser Wert wurde a } \\
\text { Pappel zwischen Okt. } 11 \text { und Okt. } 13 \text { bestimn } \\
\text { * keine signifikanten Unterschiede im }\end{array}$ & $\begin{array}{l}\text { m 6-Wocl } \\
\text {, Nov. } 201 \\
\text { löumen } \\
\text { eilfläche in } \\
\text { die ander } \\
\text { dieser W } \\
\text { chen KUP }\end{array}$ & $\begin{array}{l}\text { Turnus b } \\
\text { Okt. } 2012 \\
\text { holziges } \\
\text { tsmittelw } \\
\text { cm Bode } \\
\text { Teilfläche } \\
\text { wurde au } \\
\text { eide und A }\end{array}$ & $\begin{array}{l}\text { bt, in } 0 \text { - } \\
\text { pro Vers } \\
\text { usystem, } \\
\text { von Dez. } \\
\text { e, Sicker } \\
\text { ertragen; } \\
\text { anderen } \\
\text { eide }\end{array}$ & $\begin{array}{l}\text { Bodentiefe } \\
\text { sfläche } \\
\text { kstoff ( } N \text { ) } \\
\text { is Nov. } 12 \\
\text { serfluss wu } \\
\text { Tgasemissic } \\
\text { flächen übe }\end{array}$ & $\begin{array}{l}\text { als Gesa } \\
\text { iffenhaus } \\
\text { ur für die } \\
\text { wurden } n \\
\text { en }\end{array}$ & $\begin{array}{l}\text { he und } \\
N \text { (Holz } \\
\text { ind Jan. } \\
\text { üläche } \\
\text { ür KUP- } \\
\text { ndet }\end{array}$ \\
\hline
\end{tabular}

Die Ergebnisse der N-Aufnahmeeffizienz und N-Zuwachseffizienz für die holzigen Anbausysteme in beiden Untersuchungsregionen sind in der Tabelle 25 dargestellt. 
Tabelle 25 Stickstoffnutzungseffizienz: Stickstoffaufnahmeeffizienz und Stickstoffzuwachseffizienz [kg $\left.\mathrm{kg}^{-1}\right]$, berechnet aus dem Zuwachs an holziger Biomasse und den mittleren jährlichen Stickstoffflüssen für die verschiedenen holzigen Anbausysteme in Reiffenhausen (Reiff.) und BERTA

\begin{tabular}{|c|c|c|c|c|c|c|}
\hline & \multicolumn{2}{|c|}{ KUP-Pappel } & \multicolumn{2}{|c|}{ KUP-Weide } & \multicolumn{2}{|c|}{ AF-Weide } \\
\hline & Reiff. & BERTA & Reiff. & BERTA & Reiff. & BERTA \\
\hline $\begin{array}{l}\text { Zuwachs holzige Biomasse } \\
{\left[\mathrm{kg}_{\text {atro }} \mathrm{ha}^{-1} \mathrm{a}^{-1}\right]}\end{array}$ & 1000 & 1590 & 1090 & 590 & 930 & $590^{*}$ \\
\hline N-holzige Biomasse ${ }^{+}$ & 7,4 & 10,7 & 5,5 & 3,6 & 6,0 & $3,6^{*}$ \\
\hline$\left[\mathrm{kg}(\mathrm{N}) \mathrm{ha}^{-1} \mathrm{a}^{-1}\right]$ & $(0,9)$ & $(1,6)$ & $(0,9)$ & $(0,4)$ & $(0,7)$ & $(0,4)$ \\
\hline N-Nettomineralisation ${ }^{\wedge}$ & 18,6 & 9,0 & 28,1 & 11,8 & 23,9 & 13,7 \\
\hline$\left[\mathrm{kg}(\mathrm{N}) \mathrm{ha}^{-1} \mathrm{a}^{-1}\right]$ & $(13,3)$ & $(4,9)$ & $(25,5)$ & $(12,3)$ & $(21,4)$ & $(22,0)$ \\
\hline N-Deposition ${ }^{\circ}$ & 11,0 & 13,4 & 11,0 & 13,4 & 11,0 & 13,4 \\
\hline$\left[\mathrm{kg}(\mathrm{N}) \mathrm{ha}^{-1} \mathrm{a}^{-1}\right]$ & $(0,4)$ & $(0,6)$ & $(0,4)$ & $(0,6)$ & $(0,4)$ & $(0,6)$ \\
\hline $\begin{array}{l}\text { Stickstoffaufnahmeeffizienz ( } \mathrm{N}-\text { holzige } \\
\text { Biomasse/ [N-Nettomineralisation + N- } \\
\text { Deposition]) }\end{array}$ & 0,25 & 0,48 & 0,14 & 0,14 & 0,17 & 0,13 \\
\hline $\begin{array}{l}\text { Stickstoffzuwachseffizienz (Zuwachs holzige } \\
\text { Biomasse/ N-holzige Biomasse) }\end{array}$ & 135 & 149 & 198 & 164 & 155 & 164 \\
\hline $\begin{array}{l}\text { allometrische Biomassefunktion mit } 15 \text { Probebäum } \\
\text { und Rinde) erfasst } \\
\text { } \text { Vegetationsperiode } 2012 \text { mit Buried Bags im 6-V } \\
\text { Beprobungstermin } \\
{ }^{\circ} \text { Bulk Deposition im 2-Wochen Turnus beprobt, Nove }\end{array}$ & ochen $\mathrm{T}$ & $\begin{array}{l}\text { iges Anba } \\
\text { nus beprc } \\
\text { - Oktober }\end{array}$ & $\begin{array}{l}\text { tem, Stick } \\
\text { in } 0-30 \\
2, n=4 \text { prc }\end{array}$ & toff $(\mathrm{N}) \mathrm{w}$ & \multicolumn{2}{|c|}{$\begin{array}{l}\text { allometrische Biomassefunktion mit } 15 \text { Probebäumen pro holziges Anbausystem, Stickstoff (N) wurde als Gesamt-N (Holz } \\
\text { und Rinde) erfasst }\end{array}$} \\
\hline
\end{tabular}

Die N-Aufnahmeeffizienz ergibt sich aus dem Verhältnis von N-Entzug mit der Ernte der holzigen Biomasse (N-holzige Biomasse, Kapitel 3.2.4, Tabelle 25) pro bereitgestellten Stickstoff aus der NNettomineralisation (Kapitel 3.2.3, Tabelle 25) und der atmosphärischen N-Deposition (Kapitel 3.2.7, Tabelle 25). Auf beiden Versuchsflächen sind die N-Entzüge mit der holzigen Biomasse bei der KUPPappel stets größer als bei der KUP-Weide und AF-Weide. Die N-Nettomineralisation fällt hingegen auf beiden Versuchsflächen bei der KUP-Weide und AF-Weide größer aus als bei der KUP-Pappel. Die Gesamt-N-Deposition ist auf beiden Versuchsflächen ähnlich ausgeprägt.

Für die holzigen Anbausysteme konnte auf beiden Versuchsflächen folgende Rangfolge für die NAufnahmeeffizienz festgestellt werden:

$$
\text { KUP-Pappel > KUP-Weide AF-Weide. }
$$

Es wird ersichtlich, dass die KUP-Pappel sowohl im Vergleich zur KUP-Weide, als auch zur AF-Weide effizienter in der N-Aufnahme ist. So werden rund $25 \%$ des bereitgestellten Stickstoffs aus der NNettomineralisation und Deposition durch die Pappel bzw. 13 bis $17 \%$ durch die Weide in der holzigen Biomasse fixiert.

Die N-Zuwachseffizienz wird aus dem Verhältnis von Zuwachs der holzigen Biomasse zu N-Entzug der holzigen Biomasse gebildet (Tabelle 25). Diese kann für die holzigen Anbausysteme auf beiden Versuchsflächen wie folgt zusammenfassend geordnet werden: 
Im Hinblick auf die N-Zuwachseffizienz zeigt sich, dass die KUP-Weide und AF-Weide im Vergleich zur KUP-Pappel effizienter ist, da mit weniger Stickstoff der gleiche Zuwachs an holziger Biomasse erzielt wurde. So wurden bei der KUP-Weide bzw. AF-Weide mit 1 kg Stickstoff 155 bis $200 \mathrm{~kg}$ an holziger Biomasse produziert. Bei der KUP-Pappel konnten mit der gleichen Menge an Stickstoff 135 bis 149 $\mathrm{kg}$ an holziger Biomasse ermittelt werden.

Bei der alleinigen Betrachtung der N-Nutzungseffizienz gilt es zu beachten, dass Informationen zu NAusträgen (z.B. Sickerwasser, Lachgasemission) nicht berücksichtigt werden (Jørgensen u. Schelde 2001). So kann beispielsweise die Effizienz hoch ausfallen, aber die "Umstände" (z.B. hoher N-Austrag mit dem Sickerwasser), unter denen es zu dieser hohen Effizienz kam, werden nicht berücksichtigt wie im Fall KUP-Weide. Deshalb wurden in den vorigen Kapiteln die verschiedenen Flüsse des NKreislaufes bestimmt bzw. abgeschätzt und eine erste N-Bilanz für die verschiedenen holzigen Anbausysteme in der Etablierungsphase für beide Versuchsflächen aufgestellt.

\section{Diskussion}

\subsection{Standortcharakteristik}

Die Wasserverfügbarkeit für das Wachstum der Pflanzen wird sowohl vom Niederschlag als auch von der Wasserspeicherfähigkeit des Bodens beeinflusst. Für die klimatische Charakterisierung der Versuchsflächen wurden die Daten des DWD für die Stationen Göttingen und Dachwig genutzt. Die Station Göttingen liegt ca. $20 \mathrm{~km}$ von der Versuchsfläche Reiffenhausen entfernt. Die Station Dachwig hingegen ist ca. $3 \mathrm{~km}$ von der Versuchsfläche BERTA entfernt. Unter Berücksichtigung der Geomorphologie und der Entfernung kann davon ausgegangen werden, dass die Station Dachwig die Versuchsfläche BERTA besser repräsentiert als die Station Göttingen die Versuchsfläche Reiffenhausen.

In der "Thüringer Ackerebene" mit der Versuchsfläche BERTA reicht der Niederschlag während der Vegetationsperiode nicht aus, um hohe Erträge der schnellwachsenden Gehölze zu gewährleisten. Daher kommt der Wasserspeicherfähigkeit des Bodens eine besondere Bedeutung zu. Für BERTA konnte aufgrund der Flachgründigkeit des Bodens eine maximale Bodenwasserspeicherfähigkeit von $139 \mathrm{~mm}$ bis zur Bodentiefe von $160 \mathrm{~cm}$ ermittelt werden (Hartmann et al. 2014, Lorenz u. Müller 2013). Aus der Literatur ist bekannt, dass für ein ertragreiches Wachstum die nutzbare Wasserspeicherfähigkeit im Durchwurzelungsraum mehr als $150 \mathrm{~mm}$ betragen sollte (Petzold et al. 2010a), um gegebenenfalls Trockenphasen während der Vegetationsphase abzupuffern. Demzufolge muss die Versuchsfläche BERTA (mittlere jährliche Niederschlagsmenge Mai-September 289 mm, 1991-2010) im Hinblick auf ihre Wasserversorgung für die schnellwachsenden Gehölze kritisch 
beurteilt werden. Die Versuchsfläche Reiffenhausen wird, mit einer nachgewiesenen nutzbaren Wasserspeicherfähigkeit des Bodens von ca. $200 \mathrm{~mm}$ bis in $160 \mathrm{~cm}$ Bodentiefe (Hartmann et al. 2014) und $312 \mathrm{~mm}$ an Niederschlag in der Vegetationsperiode (mittlere jährliche Niederschlagsmenge MaiSeptember, 1991-2010), als gut wasserversorgter Standort eingeschätzt (Hartmann et al. 2014).

Für die Erfassung der bodenökologischen Bedingungen wurden zwei Methoden (Leitprofilbeprobung und Feinkartierung) angewandt, sodass auf eine große Datenmenge bis in $90 \mathrm{~cm}$ Bodentiefe zurückgegriffen werden konnte. Mit Hilfe der Feinkartierung wurde die Heterogenität in der räumlichen Verteilung der Nährstoffe für die Versuchsfläche Reiffenhausen gezeigt. Die höchsten Gehalte und Vorräte an Kalium, Magnesium, Calcium und Phosphor wurden im westlichen Bereich in Reiffenhausen beobachtet. Durch einen Wechsel im geologischen Material (Solling-Folge/Mittlerer Buntsandstein zu Röt/Oberer Buntsandstein) können diese Unterschiede erklärt werden. Höhere Tongehalte und geringe Luftkapazitäten können zu unzureichenden Umsetzungsprozessen der organischen Substanz führen, bei denen es zur Akkumulation von Stickstoff und Kohlenstoff kommt. Dies kann als möglicher Grund für die höheren Gehalte und Vorräte an $N_{t}$ und $C_{\text {org }}$ im Westen angesehen werden. Die Versuchsfläche BERTA ist durch eine homogene flächenhafte Nährstoffverteilung charakterisiert.

Die Versuchsfläche BERTA weist im Oberboden $(0-30 \mathrm{~cm})$ "sehr hohe" Calcium- und Magnesiumsowie "mittlere" Kalium- und Phosphorvorräte auf. Berücksichtigt man für die Einschätzung der Nährstoffversorgung eine Bodentiefe von beispielsweise $90 \mathrm{~cm}$ wie in Reiffenhausen, obwohl ab 50 $\mathrm{cm}$ Tiefe der Skelettgehalt auf über $50 \%$ ansteigt, wird ebenfalls ein "hoher" Vorrat an Kalium und Phosphor erreicht.

Die Ergebnisse der Feinkartierung- und der Leitprofiluntersuchung zeigen, dass die Böden nährstoffreich sind. So ergaben sich nach beiden Methoden für die Elemente Magnesium (nicht bei BERTA), Calcium, Kalium und Phosphor stets die gleichen Bewertungsklassen ("hoch" bzw. "sehr hoch"). Bei den Elementen Stickstoff und Kohlenstoff wurden mit der Leitprofilbeprobung die Vorräte über- bzw. unterschätzt, sodass im Hinblick auf genaue, repräsentative Ergebnisse eine flächenhafte Kartierung (Feinkartierung) empfohlen werden kann.

\subsection{N-Kreislauf, -Vorräte und -Flüsse}

\subsubsection{Extrahierbarer mineralischer Stickstoff}

Auf beiden Versuchsflächen wurden im Jahresmittel die höchsten Mengen an extrahierbaren mineralischem Stickstoff (eIN) auf der Ackerfläche und die geringsten auf der AF-Fläche beobachtet. Da der Großteil an elN in Form von Nitrat $(\text { eIN-NO})_{3}$ ) vorlag (außer bei der Teilfläche AF-GL in Reiffenhausen), bestand insbesondere für die Ackerböden ein hohes Verlustpotenzial, Nitrat mit dem Sickerwasser oder als Lachgas zu verlieren. 
Der Anteil an Ammonium (eIN-NH 4 ) im Ackerboden fiel in Reiffenhausen höher aus als in BERTA, was in der Ausbringung von organischem Stickstoff in Form von Mist und Gülle begründet liegt. Dem Ackerboden in BERTA wurde kein organisch gebundener Stickstoff, sondern ausschließlich mineralischer Stickstoff zugeführt, obwohl Mais organischen Stickstoff im Vergleich zu anderen Kulturen effizienter nutzt (Breitschuh u. Gernand). Da beim Maisanbau der Boden 6 bis 9 Monate unbedeckt vorliegt und somit auch keine N-Aufnahme stattfinden kann, sollten künftig Zwischenfrüchte angebaut werden, um den hohen elN-Werten entgegen zu wirken.

\subsubsection{Extrahierbarer organischer Stickstoff}

Die größten Mengen an eON wurden auf den Ackerböden in Reiffenhausen und BERTA beobachtet. In Reiffenhausen fielen die Werte höher aus als in BERTA, was mit der Applikation von organisch gebundenem Stickstoff (Mist, Gülle) begründet wird. Durch die Einarbeitung der Ernterückstände und der Düngung fielen die eON-Werte auf den Ackerflächen im Allgemeinen höher aus, im Vergleich zur KUP und dem AF.

Da sich die Vegetation auf der Teilfläche AF-GL in BERTA im Vergleich zu Reiffenhausen wesentlich schlechter entwickelte, fielen in BERTA die mittleren jährlich extrahierbaren eON-Werte geringer aus. In Reiffenhausen waren die mittleren monatlichen eON-Werte der KUP-Weide signifikant höher als in den anderen holzigen Anbausystemen. Als Ursache dafür werden die stauenden Bodenbedingungen (Sauerstoffmangel, unzureichender Umsatz der organischen Substanz) vermutet.

Mit der $\mathrm{K}_{2} \mathrm{SO}_{4}$-Extraktionsmethode werden ca. $5 \%$ des gesamten ON-Vorrats erfasst (Matsumoto u. Ae 2004, Ros et al. 2009). Im Extrakt kann sich neben mineralischem Stickstoff auch organisch gebundener Stickstoff, z.B. in Form von Aminosäuren, Proteinen etc. befinden (Jones et al. 2005). Nach Chen et al. (2013) kann der Gehalt an Aminosäuren im $\mathrm{K}_{2} \mathrm{SO}_{4}$-Extrakt ca. $25 \%$ betragen. In einigen Studien wurde nachgewiesen, dass Pflanzen in der Lage sind, freie Aminosäuren für ihre Ernährung zu nutzen (Lipson u. Näsholm 2001, Murphy et al. 2000, Neff et al. 2003). Inwieweit organischer Stickstoff z.B. in Form von Aminosäuren tatsächlich relevant zur Pflanzenernährung beiträgt, ist derzeit noch unklar (Christou et al. 2005, Jones et al. 2005, Kuzyakov u. Xu 2013, Lipson u. Näsholm 2001, Matsumoto u. Ae 2004, Murphy et al. 2000, Näsholm et al. 2009, Neff et al. 2003, Rasmussen et al. 2010, Ros et al. 2009, Schimel u. Bennett 2004).

Die eON-Menge stellt prinzipiell einen zusätzlichen N-Vorrat dar, der entweder direkt oder nach entsprechender Umwandlung (Ammonifikation, Nitrifikation) von den Pflanzen genutzt oder der Auswaschung unterliegen kann. 


\subsubsection{Stickstoff-Nettomineralisation}

Mit Hilfe der Buried Bag Methode wird die Menge an mineralischem Stickstoff (eIN) bestimmt, die für das Pflanzenwachstum zur Verfügung steht. Genaue Aussagen über die Dimension der einzelnen Flüsse, die zur Bereitstellung des elN führten (z.B. Immobilisierung, Ammonifikation, Nitrifikation), können mit dieser Methode nicht getroffen werden (Corre et al. 2006, Powlson 1993).

In den Wintermonaten wurde aufgrund des gefrorenen Bodens die Buried-Bag Methode nicht angewandt, sodass sich einzelne Datenlücken ergaben. Es wird vermutet, dass die Mineralisation aufgrund der niedrigen Temperaturen gehemmt war und somit bedeutend geringer ausfiel im Vergleich zur Vegetationsperiode (Powlson 1993), sodass eine Vernachlässigung dieser Daten zulässig erscheint.

Zwischen den verschiedenen Teilflächen konnten keine signifikanten Unterschiede in den mittleren monatlichen $\mathrm{N}$-Nettomineralisationsmengen festgestellt werden. Im Allgemeinen lagen die mittleren monatlichen $\mathrm{N}$-Nettomineralisationsmengen stets über den mittleren monatlichen elN-Mengen. Lediglich auf der Ackerfläche fielen die mittleren monatlichen eIN-Werte höher aus, da auf dieser Teilfläche eIN zusätzlich in Form von Dünger verabreicht wurde. Vergleicht man die mittleren monatlichen $\mathrm{N}$-Nettomineralisationsmengen mit den mittleren monatlichen elN-Mengen wird ersichtlich, dass die Weiden und Pappeln in KUP und AF ab April/Mai 2012 Stickstoff konsumieren und dieser sich nicht im Bodenwasser wiederfand.

In zahlreichen Studien wurde festgestellt, dass die N-Nettomineralisation von verschiedenen Faktoren abhängt, wie vom anfänglichen eON-Gehalt. So konnten hohe Mineralisationsraten in Böden mit hohen anfänglichen eON-Gehalten festgestellt werden (Friedel u. Gabel 2001, Lee u. Jose 2006). Korrelationsanalysen ließen keinen Zusammenhang zwischen den anfänglichen eON-Gehalten und der mittleren monatlichen $\mathrm{N}$-Nettomineralisationsmenge auf beiden Standorten erkennen, was zeigt, dass der eON-Vorrat vermutlich nicht die alleinige Quelle des mineralisierbaren Stickstoffs war, sondern auch gebundener Stickstoff freigesetzt wurde.

Ferner kann die N-Nettomineralisation auch vom Wassergehalt, dem $\mathrm{C}_{\text {org }}$-Gehalt und dem $\mathrm{C} / \mathrm{N}$ Verhältnis des Bodens abhängen (Devito et al. 1999, Lee u. Jose 2006, Pérez et al. 1998). In BERTA fielen die N-Nettomineralisationsmengen im Vergleich zu Reiffenhausen generell geringer aus, obwohl das mittlere C/N-Verhältnis (Reiffenhausen: 9,0, BERTA: 9,8) und der mittlere $C_{\text {org-Vorrat }}$ (Reiffenhausen: 48,4 t (C) ha ${ }^{-1}$, BERTA: 47,5 t (C) ha ${ }^{-1}$ ) im Oberboden (0-30 cm) ähnlich ausgeprägt waren. Es wird vermutet, dass aufgrund des geringeren Niederschlags in der Vegetationsperiode und der geringeren Wasserspeicherfähigkeit des Bodens in BERTA die N-Nettomineralisation durch Wasserlimitierung gehemmt war. 


\subsubsection{Holzige Biomasse}

Die Bewirtschaftung der Versuchsflächen unterschied sich zwischen den beiden Regionen. In Reiffenhausen wurde der westliche Bereich, in dem sich die KUP-Weide befindet, teilweise aus der landwirtschaftlichen Nutzung herausgenommen, sodass hier keine Düngung und Bodenbearbeitung stattfand. Dies liegt vor allem an den stauenden Bodenbedingungen, die sich aufgrund des geologischen Ausgangsmaterials ergeben. Unterschiede in der Nährstoffausstattung liegen somit vor allem in der Geologie und der unterschiedlichen Nutzung begründet. Die Versuchsfläche Reiffenhausen wurde im Winter 2010/11 mit Wintergerste bestellt, während in BERTA Winterweizen angebaut wurde. Die eigentliche Bestandsbegründung erfolgte zur selben Zeit mit der gleichen manuellen Bepflanzungsmethode. Die Begleitwuchsregulierung konnte aufgrund der intensiv geforderten Manpower und der maschinellen Ausstattung nicht einheitlich für beide Versuchsflächen erfolgen. Im Anfangsstadium reagieren Stecklinge besonders empfindlich auf Unkraut (Bowersox et al. 1992, Coll et al. 2007, Hansen u. Netzer 1985), sodass sich eine unterschiedliche mechanische und chemische Begleitwuchsregulierung im Anwuchserfolg und Ertrag widerspiegeln kann.

Unter den trockenen Bedingungen im Frühjahr 2011 (Abb. 5) konnten in Reiffenhausen höhere Anwuchserfolge bei der Weide im Vergleich zur Pappel beobachtet werden. Auf der Versuchsfläche BERTA mit ihren relativ homogenen Bodenbedingungen erreichten die Weiden ebenfalls höhere Anwuchsraten als die Pappeln, sodass im Allgemeinen davon ausgegangen werden kann, dass die Weiden in beiden Regionen besser anwachsen (Hartmann et al. 2014). Bergante et al. (2010) führt den geringen Anwuchserfolg der Pappeln (Populus deltoides) unter trockenen Bedingungen auf die geringe Fähigkeit der Pappeln zurück, Wurzeln in der Etablierungsphase zu entwickeln.

In einer Studie von Röhricht et al. (2011b) in Köllitsch (Sachsen) konnte ebenfalls unter trockenen Bedingungen in der Anwuchsphase (0,4 mm Niederschlag im April 2007) eine geringere Anwuchsrate bei der Pappel (18 \%, Klon: "Muhle Larsen", "Androscoggin") im Vergleich zur Weide (88 \%, Klon: "Inger") beobachtet werden. Demzufolge sollten die Stecklinge gerade in der Anwuchsphase mit ausreichend Wasser versorgt sein, sodass eine zusätzliche Bewässerung notwendig werden kann. In Anlehnung an Bergante et al. (2010) wird empfohlen, einen Anwuchserfolg von mehr als $80 \%$ bei den Pappelstecklingen anzustreben, damit es zu keinen Ertragseinbußen kommt.

Die Erträge wurden unter Verwendung der BHD-Werte (Durchmesser in 1,3 m Höhe) mit Hilfe der allometrischen Biomassefunktion geschätzt. Bei der Nachpflanzung waren die Ruten ca. $80 \mathrm{~cm}$ lang, sodass diese in der Biomassefunktion nicht berücksichtigt wurden. Dies führte zur allgemeinen Unterschätzung der Ertragswerte. Bei der Pappel konnten Ausfälle von $30 \%$ bis $40 \%$ beobachtet werden, bei der Weide fielen hingegen maximal $12 \%$ der Stecklinge aus. Demzufolge wurden die Erträge bei der KUP-Pappel stärker unterschätzt als bei der KUP-Weide. Ferner gestaltete sich die Erfassung der BHD aufgrund der zahlreichen Triebe bei der KUP-Weide und AF-Weide als schwierig. 
So wurde aus mehreren BHD-Triebmessungen pro Baum ein "imaginärer BHD" gebildet. Triebe, die kleiner als 1,3 m waren, wurden jedoch nicht berücksichtigt, sodass dies zu weiteren Ertragsunsicherheiten bei der Weide führte.

In Reiffenhausen wiesen die Weiden in KUP und AF signifikant größere Höhen und BHD als die Pappel auf, sodass erwartet wurde, dass diese auch signifikant höhere Erträge liefern. Stattdessen konnten für die KUP nach zweijähriger Standdauer mit Erträgen von 2,17 $t_{\text {atro }}$ ha $^{-1}$ für Weide und 2,00 $t_{\text {atro }}$ ha $^{-1}$ für Pappel kaum Unterschiede beobachtet werden. Basierend auf den Erträgen kann nach zweijähriger Standdauer keine klare Empfehlung hinsichtlich der Bevorzugung von Pappel oder Weide im Landkreis Göttingen abgeleitet werden. Es sollte jedoch berücksichtigt werden, dass in Reiffenhausen nur 63 \% der Pappeln im Vergleich zu $88 \%$ der Weiden angewachsen sind und dabei ähnlich hohe Erträge erzielt wurden.

Die Zuwächse (unter Berücksichtigung gleicher Pflanzzahlen $11.850 \mathrm{ha}^{-1}$ ) fielen mit 0,9 $\mathrm{t}_{\text {atro }} \mathrm{ha}^{-1} \mathrm{a}^{-1}$ für AF-Weide geringer aus als bei der KUP-Weide mit 1,07 $t_{\text {atro }} h^{-1} a^{-1}$. Es wird vermutet, dass insbesondere in der Etablierungsphase das Grünland aufgrund seiner Konkurrenzkraft um Wasser das Wachstum der Weiden beeinträchtigt (Stoll u. Dohrenbusch 2009). So konnten, in einer Studie von Ehret et al. (2013), die Ende 2012 für die Versuchsfläche Reiffenhausen durchgeführt wurde, signifikant geringere BHD für die Weiden (AF-Weide) im Randbereich zum Grünland (AF-GL) im Vergleich zu den Weiden innerhalb der KUP-Streifen im AF-Weide beobachtet werden.

Der Gesamtertrag vom AF ergibt sich aus den Erträgen an holziger Biomasse (AF-Weide) und der Grünlandvegetation (AF-GL). Erste Ergebnisse zum Grünlandertrag in Reiffenhausen zeigen, dass im Mittel eine jährliche Biomasseproduktion von ca. $8 \mathrm{t}$ Trockenmasse $\mathrm{ha}^{-1} \mathrm{a}^{-1}$ nach einjähriger Standdauer bzw. $11 \mathrm{t}$ Trockenmasse $\mathrm{ha}^{-1} \mathrm{a}^{-1}$ nach zwei Jahren möglich sind (Ehret et al. 2013). Unter Berücksichtigung der Flächenanteile von 55 \% für Grünland (AF-GL) und des jährlichen Zuwachses der Weide im AF (AF-Weide: $0,42 t_{\text {atro }}$ ha $^{-1} a^{-1}$, Kapitel 3.2.4) konnte ein mittlerer jährlicher Gesamtertrag von ca. 5,6 t Trockenmasse $h^{-1} a^{-1}$ für den gesamten Agroforst in Reiffenhausen für die Etablierungsphase ermittelt werden. In der Etablierungsphase wurden mit dem Anbausystem AF wesentlich höhere Biomassezuwächse als mit der KUP erzielt.

Am Standort BERTA wurden für die KUP-Weide und AF-Weide signifikant geringere Höhen und Brusthöhendurchmesser im Vergleich zur KUP-Pappel beobachtet. Der jährliche Zuwachs fiel bei der KUP-Weide $\left(0,59 t_{\text {atro }} h^{-1} a^{-1}\right)$ bedeutend geringer aus als bei der KUP-Pappel $\left(1,59 t_{\text {atro }} h^{-1} a^{-1}\right)$. Auch Biertümpfel et al. (2009) und Werner et al. (2006) stellten bei Versuchen in Thüringen fest, dass die Weide (Salix viminalis) geringere Erträge als die Pappel liefert. Aufgrund der hohen Ertragsleistungen wird in Thüringen der Anbau der Pappelklone "Max" empfohlen (Werner et al. 2006, Biertümpfel et al. 2009). 
Der Vergleich der Biomasseleistung der Weiden und Pappeln mit den Ergebnissen anderer Untersuchungen sollte mit einer gewissen Vorsicht erfolgen, da in vielen Fällen die standörtlichen (Witterung, Boden etc.) und anbautechnischen Bedingungen (Vornutzung, Vorbereitung und Bewirtschaftung der Flächen) unbekannt sind oder sich deutlich von den hier vorgefundenen unterscheiden.

Zusammenfassend lässt sich in dieser Studie und anderen deutschlandweit durchgeführten Studien feststellen, dass die Pappel in der Regel höhere Erträge als die Weide liefert (Gruenewald et al. 2007, Ihl 2010, Scholz et al. 2006).

Die geringen Erträge in der Etablierungsphase in dieser Studie liegen nicht in den geringen Nährstoffvorräten im Boden oder der mangelnden Ernährung der Bestände begründet. Bei der Auswertung der Blattanalysen wurden stets optimale bzw. ausreichende Nährstoffgehalte beobachtet. Es zeigte sich hingegen, dass die anfänglichen Biomasseerträge (die ersten zwei Standjahre) primär von der Vornutzung, Vorbereitung und Bewirtschaftung der Flächen sowie von der Witterung während der Etablierungsphase abhängen (Hartmann et al. 2014). Das Etablierungsjahr 2011 war an beiden Standorten durch eine ausgeprägte Frühjahrstrockenheit gekennzeichnet (Hartmann et al. 2014, Lorenz u. Müller 2013). Besonders in den Monaten Februar bis Mai 2011 sind teils deutlich geringere Niederschläge im Vergleich zum langjährigen Mittel gefallen. In diesem Zeitraum konnten auf beiden Versuchsflächen nur etwa $40 \%$ des langjährigen Niederschlages ermittelt werden.

Vergleichbar geringe Erträge konnten im Süden Brandenburgs beobachtet werden, auch hier wurde das Wachstum der Pappeln und Weiden durch Trockenheit in der Etablierungsphase gehemmt. Die in drei Vegetationsperioden erreichten Zuwächse für Pappel (Klone: "Japan 104", "Japan 105") und Weide (Klone: "Tordis", "Jorr", "Tora") lagen zwischen 2 und $4 t_{\text {atro }}$ ha $^{-1} a^{-1}$. Nach Landgraf et al. (2007) sollten die Ertragserwartungen an eine Kurzumtriebsplantage unabhängig vom eingesetzten Klon innerhalb der ersten Umtriebe nicht zu hoch angesetzt werden, denn unter trockenen Bedingungen investiert der Baum in den ersten Jahren primär in den Aufbau seinen Wurzelsystems und erst sekundär in die oberirdische Biomasse. Im zweiten Umtrieb soll der Ertragszuwachs dann überproportional ansteigen (Boelcke u. Kahle 2008, Hofmann-Schielle et al. 1999, Makeschin et al. 1989), sodass mit wesentlich höheren Erträgen gerechnet werden kann.

In dieser Studie wurden bei einem durchschnittlichen Zuwachs von 0,6 bis $1,6 \mathrm{t}_{\text {atro }}$ ha $\mathrm{a}^{-1} \mathrm{a}^{-1}$ in KUP nach zweijähriger Standdauer jährliche N-Entzüge von 3,6 bis $10,7 \mathrm{~kg}(\mathrm{~N}) \mathrm{ha}^{-1} \mathrm{a}^{-1}$ festgestellt. In einer Studie von Balasus et al. (2012) mit 2-jährigen Weiden- (Klon: "Inger") und Pappelklonen (Klon: "Max

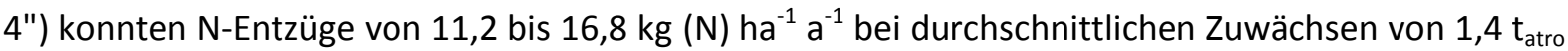
$h a^{-1} a^{-1}$ beobachtet werden. Wesentlich höhere $\mathrm{N}$-Entzüge im Vergleich zu den zuvor genannten 
Studien wurden in anderen Untersuchungen beobachtet (Adegbidi et al. 2001, Jug et al. 1999a, Scholz et al. 2006). Dies liegt in den höheren Zuwächsen bei einer deutlich längeren Standzeit der Weiden und Pappeln begründet. So betrug der jährliche N-Entzug der Pappel vom Klon "Muhle Larsen" und Weide (Salix viminalis) nach 5-jähriger Standdauer 20 bis $50 \mathrm{~kg}(\mathrm{~N}) \mathrm{ha}^{-1} \mathrm{a}^{-1}$ bei jährlichen Zuwächsen von 2 bis $8 t_{\text {atro }}$ ha $^{-1} a^{-1}$ (Bärwolff et al. 2012, Hofmann-Schielle et al. 1999, Jug et al. 1999a). Bei den anderen Elementen konnten nach 5-jähriger Standdauer Gesamtentzüge von 15 bis $45 \mathrm{~kg}(\mathrm{P}) \mathrm{ha}^{-1}$ an Phosphor, 30 bis $180 \mathrm{~kg}(\mathrm{~K}) \mathrm{ha}^{-1}$ an Kalium, 55 bis $350 \mathrm{~kg}$ (Ca) ha ${ }^{-1}$ an Calcium und 5 bis $25 \mathrm{~kg}(\mathrm{Mg}) \mathrm{ha}^{-1}$ an Magnesium bei einem Gesamtertrag von 10 bis $40 \mathrm{t}_{\text {atro }} \mathrm{ha}^{-1}$ beobachtet werden (Jug et al. 1999a). In dieser Studie betrug der Gesamtentzug an Phosphor 0,8 bis 2,8 kg (P) ha ${ }^{-1}$, Kalium 3,2 bis 10,4 kg (K) ha ${ }^{-1}$, Magnesium 0,8 bis 1,8 kg (Mg) ha ${ }^{-1}$ nach zweijähriger KUP-Standdauer und bei einem Gesamtertrag von $1,2 t_{\text {atro }}$ ha $^{-1}$ bis $3,2 t_{\text {atro }} h^{-1}$.

An weiteren Standorten z.B. in Thüringen (Dornburg, Langenwetzendorf) betrug, bezogen auf einen jährlichen Zuwachs von $1 \mathrm{t}_{\text {atro }} \mathrm{ha}^{-1} \mathrm{a}^{-1}$, der Entzug an Stickstoff rund 5,0 $\mathrm{kg}(\mathrm{N}) \mathrm{ha}^{-1} \mathrm{a}^{-1}$, Phosphor 1,0 kg (P) ha $\mathrm{h}^{-1} \mathrm{a}^{-1}$, Kalium 2,8 kg (K) ha- $\mathrm{a}^{-1}$ und Magnesium 0,6 kg (Mg) ha-1 $\mathrm{a}^{-1}$ für verschiedene 3-jährige Pappel- und Weidenklone (Werner et al. 2006). In Sachsen konnte, bezogen auf einen jährlichen Zuwachs von $1 t_{\text {atro }} h^{-1} a^{-1}$, nach zweijähriger Standdauer verschiedener Weiden- und Pappelklone ein Entzug von 6,6 kg (N) ha ${ }^{-1} a^{-1}, 1,1 \mathrm{~kg}(\mathrm{P}) \mathrm{ha}^{-1} \mathrm{a}^{-1}, 2,4 \mathrm{~kg}(\mathrm{~K}) \mathrm{ha}^{-1} \mathrm{a}^{-1}$ und 0,9 kg (Mg) ha $\mathrm{h}^{-1} \mathrm{a}^{-1}$ beobachtet werden (Röhricht et al. 2011a). Vergleicht man die Nährstoffentzüge der zuvor genannten Studien mit dieser Studie, können ähnlich hohe Werte festgestellt werden. Demzufolge scheint der Nährstoffentzug weniger vom Standort, als eher vom Alter und Zuwachs der Bestände abhängig zu sein.

Bezieht man den N-Entzug auf einen einheitlichen Zuwachs von $1 t_{\text {atro }} h^{-1} a^{-1}$, werden von der KUPPappel sowohl in Reiffenhausen als auch in BERTA höhere Mengen an Stickstoff im Vergleich zur KUP-Weide und AF-Weide entzogen. Zu ähnlichen Ergebnissen gelangt auch Werner et al. (2006) in einer Untersuchung in Thüringen. Hingegen wurden in Sachsen höhere N-Entzüge bei der Weide beobachtet (Röhricht et al. 2011a).

Vergleicht man die Entzüge bezogen, auf den Zuwachs von $1 t_{\text {atro }} h^{-1} a^{-1}$ für Kalium, Phosphor und Calcium an beiden Standorten, wird ebenfalls von der Pappel mehr entzogen als von der Weide. Dies wurde unter anderem auch von Werner et al. (2006), Röhricht et al. (2011a) und Scholz et al. (2006) bestätigt.

Durch den höheren Ertrag und der größeren Fixierung an N, K, P und Ca bei der KUP-Pappel werden dem Boden mit der Ernte der Pappelbestände generell mehr Nährstoffe im Vergleich zur KUP-Weide und AF-Weide entzogen. Dieser Sachverhalt ist für beide Standorte gültig. 


\subsubsection{Laubproduktion und Umsatz}

Im Vergleich zu anderen Studien fiel die Laubmenge nach zwei Jahren Wachstum in Reiffenhausen und BERTA mit maximal 0,9 $\mathrm{t} \mathrm{ha}^{-1} \mathrm{a}^{-1}$ bzw. 1,4 $\mathrm{tha}^{-1} \mathrm{a}^{-1}$ gering aus. So konnten für verschiedene Pappelgattungen bzw. -arten (Populus trichocarpa $x$ deltoides, Populus $x$ euramericana, Populus nigra, Populus alba) nach zweijähriger Standdauer bereits jährliche Laubfälle von 3,5 bis 5,3 t ha ${ }^{-1} \mathrm{a}^{-1}$ beobachtet werden (Cotrufo et al. 2005, Lodhiyal u. Lodhiyal 1997b). Im Allgemeinen steigt der Laubeintrag mit dem Alter und der zunehmenden Biomasseproduktion der Bäume (Berthelot et al. 2000, Guo u. Sims 1999). Die in dieser Studie beobachteten geringen Laubeinträge können demzufolge mit dem zögerlichen Wachstum der Bäume erklärt werden.

Für beide Standorte konnten die geringsten Laubeinträge im holzigen Anbausystem AF-Weide beobachtet werden. Dieser Umstand kann damit erklärt werden, dass die Bäume nur zögerlich gewachsen sind und ein Teil des Laubes in den angrenzenden Grünlandstreifen verfrachtet wurde. In einem Agroforstsystem mit 7 bis 9 Jahre alten Pappeln, die im Wechsel mit Gerste gepflanzt wurden, konnte ein Laubeintrag von ca. 2,7 tha $\mathrm{ha}^{-1} \mathrm{a}^{-1}$ in den ersten 2,5 m, ausgehend vom Pappelstreifen, in die Gerstenstreifen beobachtet werden (Thevathasan u. Gordon 1997). Informationen über den gesamten Laubeintrag innerhalb und außerhalb der Baumstreifen sind in der Untersuchung von Thevathasan u. Gordon (1997) nicht angegeben, sodass die prozentuale Verfrachtung des Laubes in die Gerstenstreifen nicht bestimmt werden konnte.

Vergleicht man die Nährstoffgehalte des Laubes in Reiffenhausen zwischen den Jahren 2011 und 2012, konnte eine allgemeine Zunahme der N- und P-Gehalte sowie eine Abnahme des K-Gehaltes mit zunehmendem Bestandsalter festgestellt werden. In BERTA hingegen nahm der N-Gehalt im gleichen Zeitraum ab, während der P- und Ca-Gehalt anstieg.

Weil die Laubmenge in der Etablierungsphase gering ausfiel, war auch die Nährstoffrückführung mit dem Laub in den Boden (Röhricht et al. 2011a) verhältnismäßig gering. In anderen Studien konnte z.B. nach vierjähriger Standdauer der Pappeln (Klon: "Beaupré"/"Raspalje") eine Nährstoffrückführung von 65,9/59,6 kg (N) ha $\mathrm{ha}^{-1}$ an Stickstoff, 11,6/10,4 kg (P) ha $\mathrm{h}^{-1} \mathrm{a}^{-1}$ an Phosphor, 41,5/35,0 kg (K) ha ${ }^{-1} a^{-1}$ an Kalium, 115,5/103,8 kg (Ca) ha ${ }^{-1} a^{-1}$ an Calcium und 9,5/8,3 kg (Mg) ha ${ }^{-1} a^{-1}$ an Magnesium beobachtet werden, bei einem Laubeintrag von 3,55/3,43 $\mathrm{tha}^{-1} \mathrm{a}^{-1}$ im vierten Standjahr (Berthelot et al. 2000). Für die beiden Versuchsflächen Reiffenhausen und BERTA standen jährlich zur Rückführung der Nährstoffe in den Boden mit dem Pappellaub 10,7/8,4 kg (N) ha ${ }^{-1} a^{-1}$ an Stickstoff, 2,9/1,1 kg (P) ha ${ }^{-1} \mathrm{a}^{-1}$ an Phosphor, 6,9/9,6 kg (K) ha ${ }^{-1} \mathrm{a}^{-1}$ an Kalium, 22,7/49,2 kg (Ca) ha ${ }^{-1}$ $\mathrm{a}^{-1}$ an Calcium und 0,9/4,5 $\mathrm{kg}(\mathrm{Mg}) \mathrm{ha}^{-1} \mathrm{a}^{-1}$ an Magnesium nach zwei Bestandsjahren zur Verfügung.

Im Vergleich mit anderen Studien kann der Gewichtsverlust des Laubes von 45 \% bis maximal $67 \%$ des Ursprungsgewichtes in Reiffenhausen und BERTA als eher hoch beurteilt werden (Albers et al. 2004, Cotrufo et al. 2005, Guo u. Sims 1999, Pérez-Corona et al. 2006). Die größten Gewichtsverluste wurden beim Pappellaub in KUP mit 53-67 \% festgestellt. Ähnlich hohe Gewichtsverluste wurden in 
den Studien von Kaushal u. Verman (2003) und Pérez-Corona et al. (2006) mit Pappellaub (Populus $x$ hybrida, Populus deltoides) und in der Studie von Šlapokas u. Granhall (1991) mit Weidenlaub (Salix viminalis, Salix daphnoides) beobachtet. Das Pappellaub wurde im Allgemeinen schneller zersetzt als das Weidenlaub, was sich in höheren jährlichen Zersetzungsraten widerspiegelt (Pappel: 1,33 bis 1,58, Weide: 1,05 bis 1,22). Ähnlich hohe jährliche Zersetzungsraten vom Pappellaub (Populus nigra: $\mathrm{k}=1,12-1,99 ;$ Populus $x$ hybrida: $\mathrm{k}=2,01$ ) wurden in den Studien von Cotrufo et al. (2005) und PérezCorona et al. (2006) ermittelt.

Im Allgemeinen wird die Laubzersetzung von zahlreichen Faktoren beeinflusst, wie z.B.

- der Laubqualität (Nährstoffgehalt, Ligningehalt, Tanningehalt) (Albers et al. 2004, Cornelissen 1996, Jacob et al. 2010, Kuers u. Simmons 2003, Pandey et al. 2007, Pérez-Corona et al. 2006, Prescott 2010, Šlapokas u. Granhall 1991, Toky u. Singh 1993),

- den klimatischen Bedingungen (Temperatur, Luftfeuchtigkeit),

- und biotischen Faktoren (Zersetzergemeinschaft) (Guo u. Sims 1999, 2001, Peh et al. 2012, Polyakova u. Billor 2007).

Unter ähnlichen klimatischen Bedingungen wird die Laubzersetzung vor allem durch die Zusammensetzung des Laubes, u.a. dessen Nährstoffgehalt, kontrolliert (Pandey et al. 2007). Da die Nährstoffgehalte $(\mathrm{N}, \mathrm{Ca}, \mathrm{Mg}$ ) im Weidenlaub höher als im Pappellaub ausfielen, könnte postuliert werden, dass dieses schneller zersetzt wird. Das Gegenteil ist jedoch eingetreten, sodass vermutlich andere Faktoren in dieser Studie maßgeblich den Streuabbau kontrollieren. In verschiedenen Studien wurde nachgewiesen, dass im Weidenlaub höhere Gehalte an Lignin und Tannin im Vergleich zum Pappellaub enthalten sind. Es wird vermutet, dass aufgrund der höheren Gehalte dieser Substanzen im Weidenlaub jenes auch langsamer zersetzt wird (Cotrufo et al. 2005, Falchero et al. 2011, Fierer et al. 2001, Gillon et al. 1999, Kaushal u. Verman 2003, Šlapokas u. Granhall 1991).

Ferner wird die Zersetzung des Laubes von der Zersetzergemeinschaft beeinflusst. Diese unterscheidet sich deutlich zwischen Acker und Wald. Im Wald wird die Zersetzergemeinschaft von Pilzen dominiert, im Acker hingegen meist von Bakterien (Stauffer et al. 2014). Dementsprechend muss sich bei der Neuanlage von KUP und AF die Zersetzergemeinschaft umstrukturieren (Baum et al. 2013, Hrynkiewicz et al. 2012, Stauffer et al. 2014). Eine besondere Herausforderung liegt dabei in der Etablierung von Organismen, die in der Lage sind, Lignin (Stauffer et al. 2014) und Tannin abzubauen - beides Substanzklassen, die deutlich weniger in der Streu klassischer Ackerfrüchte als in Pappel- und Weidenlaub vertreten sind. Wie lange es bis zum Aufbau eines Gleichgewichts zwischen mikrobieller Gemeinschaft und Streueintrag dauert, ist bisher unbekannt und Bedarf weiterer Forschung (Baum et al. 2009).

Nach einjähriger Ausbringungszeit der Litterbags wird von allen Nährstoffen Kalium mit maximal $94 \%$ am meisten dem Boden zugeführt. In zahlreichen Studien, unter anderen auch mit Weidenlaub, wurden ähnliche Ergebnisse beobachtet (Guo u. Sims 2002, Šlapokas u. Granhall 1991, Toky u. Singh 
1993). Stickstoff wurde mit ca. 30 - 40 \% nach einem Jahr deutlich weniger freigesetzt, im Vergleich zu den Nährstoffen Phosphor (außer AF-Weide Reiffenhausen), Calcium und Magnesium (außer KUPWeide Reiffenhausen und BERTA) und demzufolge stärker im Laub zurückgehalten. Trotz der schnelleren Zersetzung des Pappellaubes konnten keine signifikanten Unterschiede in der Nährstofffreisetzung (außer Ca) zwischen dem Pappel- und Weidenlaub festgestellt werden. Demzufolge werden dem Boden durch die Zersetzung des Laubes prozentual gleich große Mengen an Nährstoffen unabhängig vom Klon zurückgeführt.

Inwieweit die Zersetzung des Laubes in den Litterbags im nächsten Jahr ablaufen würde, kann nur gemutmaßt werden. In einigen Studien wurde nachgewiesen, dass die Zersetzung besonders schnell am Anfang abläuft, da zunächst die leicht zersetzbaren Bestandteile wie Zellulose abgebaut werden. Später folgen dann schwer zersetzbare Stoffe wie Lignin (Kaushal u. Verman 2003, Kuers u. Simmons 2003, Pérez-Corona et al. 2006). Demzufolge wird sich die Abbaugeschwindigkeit des Pappel- und Weidenlaubes im zweiten Jahr vermutlich verringern. In einer Studie von Pérez-Corona et al. (2006) konnte in den Litterbags mit Pappellaub (Populus $x$ hybrida), mit einer Maschenweite von $5 \mathrm{~mm}$ nach einer Ausbringungszeit von 427 Tagen, ein Restgewicht von $10 \%$ beobachtet werden. Es wird also postuliert, dass ein Großteil des Laubes innerhalb von 2 Jahren zersetzt wird. Dabei muss jedoch berücksichtigt werden, dass sich die Maschenweite der Litterbags in den einzelnen Studien voneinander unterscheidet. In einem Litterbag-Experiment mit Weidenlaub (Salix viminalis, Salix daphnoides, Salix fragilis), das von Šlapokas u. Granhall (1991) durchgeführt wurde, fiel der Gewichtsverlust mit $94 \%$ des Ursprungsgewichtes nach 406 Ausbringungstagen in den grobmaschigen Litterbags ( $4 \mathrm{~mm}$ ) höher aus als in den engmaschigen $(1 \mathrm{~mm})$ mit $53 \%$.

\subsubsection{Boden- und Sickerwasser}

Das Bodenwasser wurde mit horizontal (Ackerfläche) und vertikal (KUP, AF) eingebauten Saugkerzen gewonnen. Durch den vertikalen Einbau können präferentielle Fließwege für das Bodenwasser geschaffen werden, die eine Veränderung der Verlagerungszeit und Verlagerungsgeschwindigkeit des Bodenwassers hervorrufen. Beim horizontalen Einbau der Saugkerzen wurden Löcher gegraben, die Änderungen in der Bodenstruktur und dem Bodenwasserstrom bedingen können.

Die $\mathrm{DNO}_{3}$-Konzentrationen im Bodenwasser fielen im Vergleich zum Acker bei den holzigen Anbausystemen im Allgemeinen geringer aus. Zu ähnlichen Ergebnissen gelangte man in zahlreichen anderen Studien: so konnten in KUP stets geringere N-Konzentrationen im Bodenwasser bzw. Sickerwasser im Vergleich zu Ackerkulturen beobachtet werden (Goodlass et al. 2007, Jug et al. 1999b, Petzold et al. 2010b, Schmidt-Walter u. Lamersdorf 2012, Zacios et al. 2012). Dies liegt darin begründet, dass in KUP kein bzw. kaum Düngemittel appliziert werden (Tsonkova et al. 2012), keine wendende Bodenbearbeitung stattfindet und der Boden intensiver durchwurzelt wird (Makeschin et al. 1989), sodass auch aus tieferen Bodenbereichen Stickstoff entnommen wird (Zacios et al. 2012). 
Unmittelbar nach der Anlage einer KUP wurden in zahlreichen Studien hohe Nitratgehalte im Bodenwasser und ein verstärkter Nitrataustrag mit dem Sickerwasser beobachtet (Goodlass et al. 2007, Lamersdorf et al. 2010, Schmidt-Walter u. Lamersdorf 2012). Es kann davon ausgegangen werden, dass sich die N-Konzentrationen im Bodenwasser mit zunehmenden Alter der KUP verringern (Goodlass et al. 2007), denn im Zuge der Flächenvorbereitung und Anlage kann eine schubartige Mobilisierung und Auswaschung von leichtlöslichen N-Komponenten stattfinden (Bielefeldt et al. 2008). Die Stecklinge sind aufgrund des gering ausgebildeten Wurzelapparates in den ersten Wochen kaum in der Lage bedeutende Mengen an Stickstoff zu konsumieren (Scholz et al. 2006), sodass hier ebenfalls keine signifikante Verringerung der N-Konzentrationen im Bodenwasser erwartet wird. Durch das Ausbleiben weiterer Bodenbearbeitungen nach der Anlage der KUP bzw. $A F$, erfährt der Boden über viele Jahre hinweg eine ausgeprägte Bodenruhe (Bielefeldt et al. 2008), sodass sich die Mineralisationsraten verringern werden (Lamersdorf et al. 2010), was vermutlich eine Verringerung der N-Konzentration im Boden- bzw. Sickerwasser bedingt. Genau diese beiden Sachverhalte, die schubartige Mobilisierung und Auswaschung sowie die anschließende Abnahme von $\mathrm{DNO}_{3}$ im Bodenwasser, konnten bei allen holzigen Anbausystemen in Reiffenhausen und BERTA beobachtet werden (Abb. 18, Abb. 20).

Die höchsten Nitratkonzentrationen in den holzigen Anbausystemen wurden stets auf der Teilfläche KUP-Weide in Reiffenhausen und BERTA beobachtet. Im Vergleich zu den KUP-Flächen fielen im AF die Stickstoffkonzentrationen im Bodenwasser geringer aus. Dies lässt sich vermutlich damit erklären, dass sich das Grünland initial deutlich schneller als die Stecklinge entwickelt und somit zur Verringerung der Nitratkonzentrationen im Bodenwasser beiträgt. Durch die Fixierung von Stickstoff in die Grünland-Biomasse kann durch entsprechende Erntemaßnahmen dem Boden mehrmals pro Jahr Stickstoff entzogen werden. Inwiefern dies zur Reduktion von möglichen N-Austrägen aus dem System beitragen kann, bzw. langfristig eine negative N-Bilanz bedingt und somit eine Düngung notwendig werden lässt, kann aus der hier vorgestellten Studie nicht geschlossen werden.

\subsubsection{Deposition}

In Nordhessen, also unweit der Versuchsfläche Reiffenhausen, konnte mittels Bulk-Sammlern eine mittlere jährliche $\mathrm{DNH}_{4}$-Deposition von 4,5 bis $5,9 \mathrm{~kg}(\mathrm{~N})$ ha ${ }^{-1} \mathrm{a}^{-1}$ und eine mittlere jährliche $\mathrm{DNO}_{3}{ }^{-}$ Deposition von 4,2 bis 5,5 kg (N) ha-1 $\mathrm{a}^{-1}$ für die Messjahre 2002 bis 2004 bestimmt werden (Dämmgen et al. 2010). Die zuvor genannten Zahlen ähneln der in dieser Studie festgestellten Jahresdeposition von $11,0 \mathrm{~kg}(\mathrm{~N}) \mathrm{ha}^{-1} \mathrm{a}^{-1}$, die sich ungefähr zu gleichen Teilen auf $\mathrm{DNO}_{3}$ und $\mathrm{DNH}_{4}$ verteilt.

In der "Thüringer Ackerebene" wurde mehr $\mathrm{DNH}_{4}$ mit 7,8 kg (N) ha- $\mathrm{a}^{-1} \mathrm{im}$ Vergleich zu $\mathrm{DNO}_{3}$ mit 5,0 $\mathrm{kg}(\mathrm{N}) \mathrm{ha}^{-1} \mathrm{a}^{-1}$ deponiert. Insgesamt konnte eine jährliche Depositionsmenge von November 2011 bis Oktober 2012 von 13,4 kg (N) ha ${ }^{-1}$ beobachtet werden. Depositionsmessungen mittels Bulk- 
Sammlern werden in Thüringen von der Thüringer Landesanstalt für Landwirtschaft seit 1995 betrieben (Chmara et al. 2001). Die zur Versuchsfläche BERTA nächstgelegenen Stationen Altengottern (ca. 25 km entfernt), Großobringen und Mellingen (jeweils ca. $50 \mathrm{~km}$ entfernt) wiesen mittlere jährliche Stickstoffdepositionen von 14,1 kg (N) ha $a^{-1} a^{-1}$ für den Zeitraum 1995 bis 1999 auf, wobei der $\mathrm{DNO}_{3}$ Anteil sich auf durchschnittlich 6,8 kg (N) ha-1 $\mathrm{a}^{-1}$ und der $\mathrm{DNH}_{4}$-Anteil auf 7,3 kg (N) ha ${ }^{-1} a^{-1}$ beläuft (Chmara et al. 2001).

Die N-Gesamtdeposition wird vermutlich höher ausfallen als die hier erfasste Bulk-Deposition, da die Interzeption von Gasen und Partikeln nicht berücksichtigt wurde. Die Interzeptionsdeposition ist von der Beschaffenheit der Bäume abhängig. Da die Bäume aber noch relativ klein sind und ihre Kronenoberfläche noch nicht vollständig entwickelt ist, wird der Anteil der Interzeptionsdeposition an der gesamten Deposition vermutlich eher gering ausfallen. In einer Studie von Meiresonne et al. (2006) mit 18 Jahre alten Pappeln betrug der Anteil der Interzeption an der Gesamtdeposition hingegen bis $26 \%$.

\subsubsection{N-Bilanz und N-Nutzungseffzienz}

Zur Aufstellung der N-Bilanz wurden verschiedene Ein- und Austragsgrößen (Kapitel 2.2 \& Kapitel 3.2) erfasst, die sich jedoch auf unterschiedliche Zeitträume bezogen. Aus logistischen Gründen (zwei Versuchsflächen mit einer einfachen Entfernung von ca. $120 \mathrm{~km}$ zueinander) war leider keine zeitgleiche Beprobung möglich. Ferner erfolgte die Bestimmung der N-Gehalte im Boden und Bodenwasser auf relativ kleinen Beprobungsflächen. Demzufolge wurden stets nur punktuelle Proben gewonnen, mit denen nur kleine Ausschnitte des Gesamtsystems erfasst wurden. Gerade der Standort Reiffenhausen ist durch eine hohe räumliche Heterogenität in der Nährstoffausstattung des Bodens gekennzeichnet. BERTA weist hingegen relativ homogene Bodenbedingungen auf, sodass gleiche Ergebnisse, die im Hinblick auf den N-Kreislauf auf beiden Flächen beobachtet wurden, als standortunabhängig angesehen werden können.

Auf beiden Versuchsflächen wurden höhere N-Nettomineralisationsmengen unter der KUP-Weide und AF-Weide im Vergleich zur KUP-Pappel ermittelt. Es wird vermutet, dass die biologische Aktivität in den Weidensystemen aufgrund des höheren Anwuchserfolges intensiver ist als im Vergleich zur KUP-Pappel. In diesem Zusammenhang können in den holzigen Anbausystemen mit Weide im Allgemeinen höhere elN-Mengen im Boden und höhere N-Austräge mit dem Sickerwasser beobachtet werden. Aufgrund der hohen N-Austräge mit dem Sickerwasser ergibt sich für die KUPWeide eine negative N-Bilanz. Demzufolge wird dem System im Laufe eines Jahres mehr Stickstoff entzogen, als mit der N-Nettomineralisation und der Deposition bereitgestellt wird. Hält dieses Phänomen langfristig an, wird sich der N-Vorrat und somit die Fruchtbarkeit des Bodens verringern (Kölling et al. 2007). Damit die holzigen Anbausysteme (KUP und AF) auf landwirtschaftlichen Nutzflächen möglichst langfristig und demzufolge nachhaltig funktionieren, sollte eine nahezu 
ausgeglichene N-Bilanz angestrebt werden. Es wird vermutet, dass sich die N-Austräge mit dem Sickerwasser verringern werden, sodass auch die KUP-Weide künftig eine ausgeglichene oder positive N-Bilanz wie die anderen holzigen Anbausysteme aufweist. Die größten Mengen an Stickstoff werden mit rund $20 \mathrm{~kg}(\mathrm{~N}) \mathrm{ha}^{-1} \mathrm{a}^{-1} \mathrm{im}$ AF-Weide akkumuliert.

Bereits der jährliche N-Eintrag mit der Deposition ist ausreichend, um den Stickstoffbedarf der holzigen Biomasse in der Etablierungsphase zu decken, sodass keine Düngung notwendig ist. $\mathrm{Zu}$ diesem Ergebnis gelangten auch Bärwolff et al. (2012), Balasus et al. (2012), Bielefeldt et al. (2008), Scholz et al. (2006) und Werner et al. (2006).

Künftig wird die Produktion an holziger Biomasse überproportional steigen (Boelcke u. Kahle 2008, Volk et al. 2011). Unter der Annahme, dass im Holz inkl. Rinde bei einem Ertrag von $11 \mathrm{t}_{\text {atro }} \mathrm{ha}^{-1} \mathrm{a}^{-1}$ rund $15 \mathrm{~kg}(\mathrm{~N}) \mathrm{ha}^{-1} \mathrm{a}^{-1}$ an Stickstoff gespeichert wird (Röhricht $\mathrm{u}$. Ruscher 2009), können mit der jährlichen Deposition in Reiffenhausen rund 73 \% bzw. 89 \% des N-Entzugs in BERTA gedeckt werden. Inwieweit sich die Lachgasemissionen der KUP-Weide und AF-Weide tatsächlich von der KUP-Pappel unterscheiden, kann an dieser Stelle nur geschätzt werden. Im Vergleich zur KUP-Pappel fallen die elN-Mengen und die $\mathrm{DNO}_{3}$ Konzentrationen in der AF-Weide geringer aus, sodass die Annahme einer maximalen Lachgasemission von $2 \mathrm{~kg}(\mathrm{~N}) \mathrm{ha}^{-1} \mathrm{a}^{-1}$ für Reiffenhausen (2011-2012) und von $1 \mathrm{~kg}$ (N) ha ${ }^{-1} a^{-1}$ für BERTA (2011 bis 2013) legitim erscheint. Auf der KUP-Weide wird der Lachgasverlust vermutlich am höchsten ausfallen, hervorgerufen durch höhere elN-Mengen im Boden und höhere $\mathrm{DNO}_{3}$-Konzentrationen im Bodenwasser. Ferner wird vermutet, dass die Lachgasemission der KUPWeide in Reiffenhausen aufgrund der Bodenbedingungen (Staunässe, Sauerstoffmangel) im Vergleich zur KUP-Weide in BERTA höher ist.

Unter Berücksichtigung der N-Nutzungseffizienz kann davon ausgegangen werden, dass die holzigen Anbausysteme mit Weide effizienter sind als die KUP-Pappel, denn mit gleicher N-Menge (z.B. aus dem Boden) wird von der Weide mehr holzige Biomasse im Vergleich zur Pappel produziert. Demzufolge benötigt die Pappel mehr Stickstoff, um die gleiche Menge an holziger Biomasse wie die Weide zu produzieren.

Aus der Kombination der N-Effizienz, der N-Flüsse und der aufgestellten N-Bilanz in der Etablierungsphase können folgende Schlüsse zu den holzigen Anbausystemen in Reiffenhausen und BERTA gezogen werden:

- das holzige Anbausystem AF-Weide weist den höchsten Überschuss an Stickstoff in der NBilanz im Vergleich zu den anderen holzigen Anbausystemen KUP-Pappel bzw. KUP-Weide auf, verursacht durch:

- eine hohe N-Nettomineralisation in der Vegetationsperiode,

- einen geringen N-Austrag mit der holzigen Biomasse und dem Sickerwasser,

- eine hohe N-Zuwachseffizienz, 
- die geringsten Werte in der N-Bilanz wurden im holzigen Anbausystem KUP-Weide festgestellt, verursacht durch:

- große N-Austräge mit dem Sickerwasser.

\section{Schlussfolgerungen und Empfehlungen für die Praxis}

Zur Steigerung der regionalen Biomasseproduktion wurden im Rahmen des Verbundprojektes "Bioenergie-Regionen stärken" (BEST) Kurzumtriebsplantagen und Agroforstsysteme in den beiden Bioenergie-Regionen "Göttinger Land" und "Thüringer Ackerebene" angelegt. Als schnellwachsende Gehölze wurden der Pappelklon "Max 1" und der Weidenklon "Tordis" genutzt. Beide Klone sind durch eine hohe Anwuchssicherheit und große Ertragszuwächse charakterisiert (Biertümpfel et al. 2009, Boelcke 2006, Hofmann et al. 2012). Für das Erreichen hoher Erträge werden gewisse Anforderungen an die Versuchsflächen in beiden Regionen gestellt. So sollten die Jahrestemperatur mehr als $6,5^{\circ} \mathrm{C}$ (Aust 2012, Petzold et al. 2010a), die Jahresniederschlagssumme mehr als $500 \mathrm{~mm}$ und die Niederschlagssumme zwischen Mai und September mehr als $300 \mathrm{~mm}$ betragen (Petzold et al. 2006, Petzold et al. 2010a). Ferner sollte das Nährstoffangebot hoch sein (Liebhard 2007, Petzold et al. 2006). Die beiden Bodenuntersuchungen belegen, dass die Böden der beiden Versuchsflächen nährstoffreich sind und somit ein ertragreiches Wachstum der Gehölze über Jahre hinweg ermöglichen. Die pH-Werte liegen im neutralen bis schwach sauren Bereich. Die Vorräte an austauschbar gebundenen basischen Kationen liegen meistens nahe $100 \%$. Lediglich die Versuchsfläche im Ldk. Gö. Reiffenhausen lässt eine schwache Versauerungstendenz erkennen, die sich aber positiv auf die Phosphor- und Kaliumverfügbarkeit auswirken kann. Insgesamt sind an beiden Versuchsflächen keine durch Nährstoffmangel bedingten Wachstumseinschränkungen der Weiden und Pappeln zu erwarten. Dies bestätigen ebenfalls die in dieser Studie durchgeführten Untersuchungen der Blattnährstoffgehalte.

Auch die mittlere Lufttemperatur $\left(>6,5^{\circ} \mathrm{C}\right)$ und die Jahresniederschlagssumme $(>500 \mathrm{~mm}$ ) können in beiden Regionen günstig beurteilt werden. Allerdings wird die geforderte Niederschlagsmenge in der Vegetationszeit (Mai bis September $>300 \mathrm{~mm}$ ) auf der Versuchsfläche in der "Thüringer Ackerebene" unterschritten (langjähriges Mittel: $289 \mathrm{~mm}$ ), sodass die Rolle des Bodens als Wasserspeicher an Bedeutung gewinnt. Für die Versuchsfläche BERTA wurde eine nutzbare Wasserspeicherfähigkeit des Bodens von $139 \mathrm{~mm}$ bis in 1,6 m Bodentiefe ermittelt. Eine nutzbare Wasserspeicherfähigkeit von weniger als $150 \mathrm{~mm}$ in Kombination mit einer Niederschlagsmenge unter $300 \mathrm{~mm}$ in der Vegetationsperiode führt dazu, dass die Anlage von KUP in BERTA nicht empfohlen werden kann, sofern kein Anschluss an das Grundwasser besteht. Die Versuchsfläche im Ldk. Gö. verfügt über eine hohe nutzbare Wasserspeicherfähigkeit $(196 \mathrm{~mm})$. Bei gleichzeitig ausreichenden 
Niederschlägen in der Vegetationszeit (langjähriges Mittel: $312 \mathrm{~mm}$ ) kann hier der Anbau von schnellwachsenden Gehölzen uneingeschränkt empfohlen werden.

Betrachtet man die anfänglichen Biomasseerträge als Maß für eine angemessene Standortwahl, so ergibt sich aus den vorliegenden Ergebnissen folgendes Bild: Auf der Versuchsfläche im Ldk. Gö. wurden unter den trockenen Bedingungen des Frühjahrs 2011 höhere Anwuchserfolge bei der KUPWeide und AF-Weide im Vergleich zur KUP-Pappel festgestellt. Gleiches konnte auf der Versuchsfläche in der "Thüringer Ackerebene" mit ihren relativ homogenen Bodenbedingungen beobachtet werden, sodass im Allgemeinen davon ausgegangen werden kann, dass der Weidenklon "Tordis" in beiden Regionen besser anwächst, als der Pappelklon "Max 1". Im Ldk. Gö. konnten in den angelegten KUP nach zweijähriger Standdauer Erträge von 1,09 $t_{\text {atro }}$ ha $^{-1} a^{-1}$ für Weide und 1,00 $t_{\text {atro }} h^{-1} a^{-1}$ für Pappel ermittelt werden. Zum bisherigen Zeitpunkt kann keine klare Empfehlung hinsichtlich der Bevorzugung von Pappel oder Weide für den Ldk. Gö. gegeben werden. Es sollte jedoch berücksichtigt werden, dass die Pappeln einen vergleichbaren Ertrag wie die Weiden im Beobachtungszeitraum erzielten, obwohl nur $63 \%$ der Pflanzen angewachsen sind.

In der "Thüringer Ackerebene" konnten Anwuchsraten von mehr als $90 \%$ bei der Weide in KUP und AF beobachtet werden, die Erträge fielen auf der KUP-Weide mit 0,59 $t_{\text {atro }} h^{-1} a^{-1}$ im Vergleich zur KUP-Pappel mit 1,59 $t_{\text {atro }}$ ha $^{-1} a^{-1}$ bei einer Anwuchsrate von nur $74 \%$ gering aus. Demzufolge erscheint der Weidenklon "Tordis" zur ertragreichen initialen Biomasseproduktion in der "Thüringer Ackerebene" ungeeignet. Für ein erfolgreiches Wachstum der Gehölze ist demzufolge eine ausreichende initiale Wasserversorgung bedeutsam, sodass Bewässerungsmaßnahmen, wie in einem weiteren Teilprojekt ("Bewässerung", Bearbeiter Konrad Lorenz, Waldökosysteme, vTi Eberswalde) von BEST bereits durchgeführt, als mögliche Option zum Erreichen hoher Anwuchserfolge und Erträge geprüft werden sollte.

Wie der Vergleich der Ertragsdaten zwischen KUP-Weide und KUP-Pappel belegt, sollten bei der Neuanlage von KUP sämtliche Maßnahmen ergriffen werden, um hohe Anwuchsraten zu sichern. In Anlehnung an Bergante et al. (2010) wird empfohlen, einen Anwuchserfolg von mehr als $80 \%$ bei den Pappelstecklingen anzustreben, damit es zu keinen Ertragseinbußen kommt. Als entscheidende Einflussfaktoren für das Erreichen hoher Anwuchsraten und Erträge gelten die Witterung im ersten Frühjahr, die sorgfältige Flächenvorbereitung und Begleitwuchsregulierung. Nur wenn die Stecklinge über genügend Wasser, Licht und Nährstoffe verfügen, können sie hohe Biomasseerträge liefern.

Für KUP und AF ergeben sich andere Vorräte und Flüsse an Stickstoff im Vergleich zu den landwirtschaftlichen Kulturen. Erwartungsgemäß wurden die größten Mengen an jährlich extrahierbarem mineralischen und organischen Stickstoff im Boden in beiden Regionen auf der Referenzfläche Acker beobachtet. Da der Großteil an extrahierbarem mineralischen Stickstoff in Form von Nitrat vorlag, besteht insbesondere für die Ackerfläche ein hohes Potenzial, Stickstoff mit dem Sickerwasser zu verlieren. Tatsächlich konnten die größten Austräge bei der KUP-Weide 
beobachtet werden. Die geringsten Mengen an extrahierbarem mineralischen Stickstoff im Boden und Sickerwasser konnten im AF festgestellt werden. Demzufolge erscheinen Grünlandstreifen zwischen der KUP-Weide in Form von AF als sinnvolle Maßnahme, um den N-Austrag mit dem Sickerwasser zu verringern. Im Hinblick auf geringe N-Austräge mit dem Sickerwasser kann als holziges Anbausystem zur Biomasseproduktion die Anlage von AF empfohlen werden.

Steht insbesondere die Produktion an holziger Biomasse in KUP als mögliches Anbauziel im Vordergrund, sollte der Pappelklon "Max 1" im Vergleich zum Weidenklon "Tordis" gewählt werden. So konnten in dieser Studie die höchsten Holzerträge bei der KUP-Pappel beobachtet werden. In Thüringen fielen die Erträge der KUP-Pappel mehr als doppelt so hoch aus als bei der KUPWeide. Auch die Laubproduktion fiel bei der KUP-Pappel signifikant höher aus im Vergleich zu den anderen holzigen Anbausystemen. Die höchsten Laubeinträge wurden stets bei der KUP-Pappel festgestellt, sodass absolut gesehen, mit dem Pappellaub auch die größten Nährstoffmengen dem Boden entzogen und später in organisch gebundener Form erneut zugeführt werden. Die Zersetzung des Laubes verlief beim Pappellaub im Vergleich zum Weidenlaub schneller, sodass für eine möglichst rasche Nährstoffrückführung und C-Sequestrierung im Boden die Pappel bevorzugt angebaut werden sollte.

Um die verschiedenen holzigen Anbausysteme im Hinblick auf ihre Nachhaltigkeit zu beurteilen und Empfehlungen zur Wahl des holzigen Anbausystems in der Etablierungsphase zu geben, wurde die NNutzungseffizienz berechnet und die initiale N-Bilanz aufgestellt. Ein Ökosystem kann als nachhaltig angesehen werden, wenn ein nahezu geschlossener N-Kreislauf mit ausgeglichener N-Bilanz vorliegt (Kölling et al. 2007). Es muss dabei berücksichtigt werden, dass dem holzigen Anbausystem im regelmäßigen Rhythmus Stickstoff mit der Holzernte entzogen wird. Werden diese N-Verluste nicht in gleicher Größenordnung ausgeglichen, wird sich der N-Vorrat und somit die Fruchtbarkeit des Bodens verringern (Kölling et al. 2007). Die vorliegenden Untersuchungen haben bestätigt, dass allein der jährliche N-Eintrag mit der Deposition ausreicht, um den N-Bedarf der Pappeln und Weiden zur Produktion von holziger Biomasse zu decken.

In der Literatur werden KUP häufig als Anbausysteme mit geschlossenem N-Kreislauf charakterisiert, d.h. trotz N-Eintrag mit der Deposition können keine Akkumulationen und unerwünschten Austräge beobachtet werden (Strohm et al. 2012). Aus den aufgestellten initialen N-Bilanzen in dieser Studie wird hingegen ersichtlich, dass die Etablierungsphase von KUP und AF nicht durch einen geschlossenen $\mathrm{N}$-Kreislauf charakterisiert ist.

Im Hinblick auf die N-Nutzungseffizienz wird die Weide besser bewertet als die Pappel, denn mit gleicher Stickstoffmenge (z.B. aus dem Boden) wird von der Weide mehr holzige Biomasse produziert. Im Falle geringer bodenbürtiger Vorräte an Stickstoff sollten im Hinblick auf die Stickstoffnutzungseffizienz die holzigen Anbausysteme KUP-Weide bzw. AF-Weide bevorzugt angebaut werden. 
Um ggf. einen vorhandenen Überschuss an Stickstoff aus der ehemaligen Düngung oder sonstigen Einträgen (z.B. aus der Deposition) zu reduzieren und dabei viel Biomasse in der Etablierungsphase zu produzieren, kann aus den hier gefundenen Ergebnissen die KUP-Pappel als holziges Anbausystem empfohlen werden. Inwieweit sich durch die Kombination von Pappel im Wechsel mit Grünland mögliche Synergien im Hinblick auf die N-Bilanz oder Biomasseproduktion ergeben, könnte Gegenstand künftiger Forschung werden.

\section{Zusammenfassung}

Vor dem Hintergrund des Klimawandels und der Verknappung an fossilen Energieträgern soll der Anteil der erneuerbaren Energien am Endenergieverbrauch in Deutschland gesteigert werden. Dabei kann die Biomassenutzung wesentlich zur Sicherung der Energieversorgung beitragen. Im Rahmen der Untersuchungen zur Steigerung der regionalen Biomasseproduktion wird seit Ende 2010 das Verbundprojekt "Bioenergie-Regionen stärken" (BEST) durchgeführt. Das Ziel von BEST ist es, regional angepasste Konzepte und innovative Systemlösungen zur Produktion von Biomasse zu entwickeln und im Hinblick auf ökologische und ökonomische Auswirkungen anhand zweier Bioenergie-Regionen ("Göttinger Land", Südniedersachsen und "Thüringer Ackerebene", Mittelthüringen) zu bewerten. Das BEST-Teilprojekt "Agroforst und Kurzumtriebsplantagen", bildet die Grundlage für die vorliegende Arbeit und untersucht innovative Anbausysteme zur Gewinnung von holziger Biomasse.

Die Hauptziele dieser Studie sind:

1. Entwicklung von regional angepassten Konzepten und innovativen Systemlösungen zur Produktion von Energieholz mittels Kurzumtriebsplantagen (KUP) und Agroforstsystemen (AF). Dabei versteht man unter KUP den Anbau von schnellwachsenden Gehölzen wie Pappel und Weide auf landwirtschaftlichen Flächen. Eine spezielle Variante des KUP-Anbaus sind so genannte Agroforstsysteme, eine Mischung von KUP-Streifen mit Ackerkulturen oder Grünland auf der gleichen landwirtschaftlichen Fläche.

2. Bereitstellung von praxisrelevanten Versuchs- und Demonstrationsflächen mit KUP und AF in den beiden Bioenergie-Regionen "Göttinger Land" und "Thüringer Ackerebene".

3. Bodenökologische Charakterisierung der Versuchs- und Demonstrationsflächen.

4. Ableitung von Empfehlungen für die Praxis.

Zur Konkretisierung der Hauptziele wurden folgende Teilziele formuliert: 
- Bereitstellung, Analyse und Bewertung von Daten zu den klimatischen, geologischen, bodenphysikalischen und bodenchemischen Ausgangsbedingungen der Versuchsflächen in den beiden Bioenergie-Regionen.

- Analyse des Ernährungszustandes der schnellwachsenden Gehölze.

- Erfolgskontrolle durch Erfassung der Anwuchs- und Ertragsentwicklung.

- Vergleichende Bestimmung der Laubproduktion, des Laubumsatzes und der Nährstofffreisetzung für die eingesetzten schnellwachsenden Gehölze.

- Abschätzungen zu Vorräten und Flüssen des initialen Stickstoffkreislaufes.

- Integrierte Bewertung der Versuchsflächen hinsichtlich ihrer Eignung für den Anbau von KUP und $\mathrm{AF}$.

Aus der Kombination verschiedener Disziplinen (u.a. Meteorologie, Geologie, Bodenkunde, Geographie), Datenquellen (u.a. langjährige klimatische Informationen des Deutschen Wetterdienstes) und Erhebungen (z.B. Bodenfeinkartierung, Bodenleitprofile, Blattnährstoffgehalte, Buried Bags, Deposition) wurden die ausgewählten Versuchsflächen naturräumlich charakterisiert. Weiterhin wurde eine Beurteilung der Standorte im Hinblick auf deren Eignung zur Anlage von schnellwachsenden Gehölzen (Pappelklon "Max 1" und Weidenklon "Tordis") vorgenommen.

Zur Charakterisierung des Wasserhaushaltes der Versuchsflächen dienten verschiedene klimatische und bodenphysikalische Parameter. Im Detail konnte aus der Kombination der langjährigen Niederschlagssumme innerhalb der Vegetationsperiode und der nutzbaren Wasserspeicherfähigkeit des Bodens geschlossen werden, dass die Integration von KUP und AF in die "Thüringer Ackerebene" nicht empfehlenswert ist. Hingegen kann aus Sicht der Wasserversorgung die Anlage von KUP und AF auf der Versuchsfläche im "Göttinger Land" uneingeschränkt empfohlen werden. Die Ergebnisse der geologischen und bodenchemischen Untersuchung zeigen, dass beide Versuchsflächen nährstoffreich sind und sich demzufolge für einen ertragreichen Anbau der Pappel- und Weidenklone eignen. Durchgeführte Blattanalysen nach ein- bzw. zweijähriger Standdauer belegen, dass den Gehölzen für ihr ertragreiches Wachstum, genügend Nährstoffe zur Verfügung standen.

Dennoch wurden nach zweijähriger Standdauer nur geringe Erträge von maximal 3,2 $\mathrm{t}_{\text {atro }} \mathrm{ha}^{-1}$ mit Hilfe der allometrischen Biomassefunktion ermittelt. Mit dem Pappelklon "Max 1" konnten, unter Berücksichtigung der Anwuchserfolge, höhere Erträge im Vergleich zum Weidenklon "Tordis" festgestellt werden. Die Anwuchsrate der Weide ist mit ca. $90 \%$ wesentlich höher ausgefallen als bei der Pappel mit maximal $74 \%$.

Die Ergebnisse zur Laubproduktion, des Laubumsatzes und der Nährstofffreisetzung zeigen, dass signifikant mehr Laub vom Pappelklon "Max 1" im Vergleich zum Weidenklon "Tordis" produziert wurde und dass das Pappellaub wesentlich schneller zersetzt wurde. Demzufolge gelangen mit dem Pappellaub mehr Nährstoffe in den Boden als mit dem Weidenlaub. 
Zur Untersuchung des initialen Stickstoffkreislaufes (N-Kreislaufes) wurden verschiedene N-Flüsse und -Vorräte bestimmt, die N-Nutzungseffizienz ermittelt und eine N-Bilanz für KUP und AF in den beiden Regionen aufgestellt. Als Eintragsgrößen gingen in die N-Bilanz die N-Nettomineralisation und $\mathrm{N}$-Deposition ein. Die N-Nettomineralisationsmengen unterschieden sich nicht signifikant zwischen KUP, AF und der Kontrollfläche Acker. Die Jahresdeposition an $\mathrm{N}$ betrug auf beiden Versuchsflächen ca. 11 bis $13 \mathrm{~kg}(\mathrm{~N}) \mathrm{ha}^{-1}$ und fiel demzufolge zwischen den beiden Regionen ähnlich aus.

Als Austragsgrößen gingen in die N-Bilanz der Nitrataustrag mit dem Sickerwasser, der Lachgasverlust und die Ernte der holzigen Biomasse ein. Die Nitratausträge wiesen insbesondere unmittelbar nach der Anlage von KUP bzw. AF hohe Werte auf, die sich im Laufe des Jahres verringerten. Die größten Nitratausträge mit dem Sickerwasser wurden unabhängig vom Standort für die Weide in KUP beobachtet. Der N-Entzug mit der Ernte der holzigen Biomasse fiel am größten bei der Pappel in KUP aus, verursacht durch höhere Nährstoffgehalte und Erträge im Vergleich zur Weide. Die Lachgasemissionen betrugen für die Pappel in KUP maximal $2 \mathrm{~kg} \mathrm{ha}^{-1} \mathrm{a}^{-1}$ auf beiden Versuchsflächen.

Unter Berücksichtigung der gesamten $\mathrm{N}$-Eintrags- und $\mathrm{N}$-Austragsflüsse ergaben sich $\mathrm{N}$-Bilanzen von $-14,7 \mathrm{~kg}(\mathrm{~N}) \mathrm{ha}^{-1} \mathrm{a}^{-1}$ bis $22,5 \mathrm{~kg}(\mathrm{~N}) \mathrm{ha}^{-1} \mathrm{a}^{-1}$ für KUP und AF auf beiden Versuchsflächen in der Etablierungsphase. Im "Göttinger Land" wurden ausschließlich positive N-Bilanzen ermittelt. Auf der Versuchsfläche in der "Thüringer Ackerebene" ergaben sich hingegen negative Bilanzen für KUP mit Pappel und Weide. Positive Bilanzen bedeuten, dass trotz N-Austrägen mit dem Sickerwasser, Lachgas und der Ernte der holzigen Biomasse Stickstoff im System zurückgehalten wird.

Unter Berücksichtigung der N-Nutzungseffizienz wurde festgestellt, dass von der Weide, unter Einsatz gleicher N-Mengen, mehr holzige Biomasse produziert wurde, im Vergleich zur Pappel.

Im Falle geringer bodenbürtiger N-Vorräte sollte im Hinblick auf die N-Nutzungseffizienz die Weide bevorzugt angebaut werden. Der Anbau des Pappelklons "Max 1" in KUP kann empfohlen werden, um einen vorhandenen Überschuss an Stickstoff aus der ehemaligen Düngung oder sonstigen Einträgen (z.B. aus der Deposition) zu minimieren und dabei viel Biomasse in der Etablierungsphase zu produzieren.

Die Versuchsfläche im "Göttinger Land" kann aus der Kombination der naturräumlichen Charakterisierung und der N-Bilanz als Anbaufläche von KUP und AF empfohlen werden.

\section{Summary}

In the context of climate change and the decrease of fossil fuels the production of renewable energies should be extended. The use of biomass is one option to help secure the energy supply. 
Based on the need for regional increase of biomass production for material and energetic use the research framework BEST - for the support of bioenergy regions - is being conducted since 2010. One focus of BEST is set on the development of regionally adapted concepts and innovative systems for the production of woody biomass taking into account ecological and economic effects. Research activities of BEST were linked to two bioenergy regions: the district of Göttingen (Ldk. Gö.) located in Southern Saxony and the initiative "BERTA" (BioEnergy Region Thuringian cropping Area) in the center of the Thuringian cropping area. The subproject "Agroforestry and short rotation coppices" of BEST as the base of this study analyses innovative systems for the production of woody biomass.

Main goals of the study are:

1. the development of regionally adapted concepts and innovative systems for the production of woody biomass within the land use systems short rotation coppice (SRC) and agroforestry (AF). SRC provide woody biomass with fast growing trees, like poplar or willow, on agricultural land. The combination of SRC rows and grassland or arable crops on the same agricultural site are called AF.

2. Establishment of practical SRC and AF study sites in both bioenergy regions Ldk. Gö. and BERTA.

\section{Soil ecological characterization of the study sites.}

4. Deduction of practical recommendations.

Furthermore, the following subgoals were identified for achieving the main goals:

- provision, analysis and assessment of geological, climatic, soil physical and soil chemical data for the study sites in both bioenergy regions,

- analysis of the nutritional status of the fast growing trees,

- verification of success by gathering information on growth and yield,

- measurement and comparison of litter production, litter loss and nutrient release for the used clones,

- estimation of fluxes and pools of initial nitrogen cycling,

- integrated assessment of suitability for the study sites concering KUP and AF.

Several disciplines (meteorology, soil science, geology, geography), data (long term data from the German Weather Service) and observations (detailed soil mapping, soil profiles, leaf nutrient content, buried bags, deposition) were used for evaluation of the study sites with fast growing trees (poplar clone "Max 1" and willow clone "Tordis").

For characterization of water supply climatic and soil physical data were used. Based on the results of the long term mean precipitation and the available water capacity of soil an establishment of SRC and AF in BERTA cannot be recommended. However, the planting of SRC and AF can fully be recommended in Ldk. Gö. Geological and soil chemical data indicate good nutrient conditions for plant growth of poplar and willow. However, low initial biomass yields, with a maximum of 
$3.2 t_{\text {atro }}$ ha $a^{-1} a^{-1}$ were calculated for both study sites with the help of allometric power equations. Survival rates and initial biomass yields were higher by poplar in comparison to willow. Especially the survival rates of willow (approx. $90 \%$ ) were higher than for poplar (maximum of $74 \%$ ).

Further litter production, decomposition and nutrient release was higher for poplar clone "Max 1" in comparison to willow clone "Tordis". Therefore, more nutrients are returned with poplar litter into the soil in comparison to willow.

Initial nitrogen $(\mathrm{N})$ cycling, fluxes and pools of $\mathrm{N}$ and $\mathrm{N}$ use efficiency were analyzed for SRC and $\mathrm{AF}$ in both regions. Fluxes of mean Net-N-Mineralization (NNM) plus the measured atmospheric $\mathrm{N}$ bulk deposition were taken as the input terms. Using buried bags for NNM, no significant differences between SRC, AF and agricultural control plot were detected. Atmospheric $\mathrm{N}$ deposition was very similar for both regions (11 - $\left.13 \mathrm{~kg}(\mathrm{~N}) \mathrm{ha}^{-1} \mathrm{a}^{-1}\right)$.

Output terms for $\mathrm{N}$ were defined as the plant uptake in wood, including bark, plus the losses of $\mathrm{N}$ by nitrate leaching and $\mathrm{N}_{2} \mathrm{O}$ emissions. High nitrate leaching was especially determined immediately after establishment of SRC and AF but decreased towards the end of the observation period. Highest nitrate leaching was observed for willow in SRC on both study sites. Plant uptake of $\mathrm{N}$ in wood, including bark was highest in poplar SRC, mainly caused by higher nutrient contents and biomass yields in comparison to willow. $\mathrm{N}_{2} \mathrm{O}$ emissions for poplar SRC were determined up to a maximum of 2 $\mathrm{kg} \mathrm{ha}^{-1} \mathrm{a}^{-1}$ in both regions.

Initial N-budgets for all plots (considering the input and output fluxes) ranged between $-14.7 \mathrm{~kg}(\mathrm{~N})$ $\mathrm{ha}^{-1} \mathrm{a}^{-1}$ and $22.5 \mathrm{~kg}(\mathrm{~N}) \mathrm{ha}^{-1} \mathrm{a}^{-1}$ for SRC and AF. In Ldk. Gö., SRC and AF always showed positive Nbudgets whereas in BERTA, negative N-budgets were calculated for SRC with willow and SRC with poplar. A positive $\mathrm{N}$-budget indicates an accumulation of $\mathrm{N}$ despite of nitrate leaching, $\mathrm{N}$-uptake in wood and $\mathrm{N}_{2} \mathrm{O}$ emissions.

Willow seems to be more efficient than poplar with respect to $\mathrm{N}$ use efficiency. A higher biomass production was observed for willow in comparison to poplar considering the same amount of nitrogen, which means willow is more effective in the use of nitrogen. Considering low stock of nitrogen in soil, willow is the most $\mathrm{N}$-efficient system to produce woody biomass. Poplar should be used if the main goal is diminishing $\mathrm{N}$ eutrophication and yielding high woody biomass.

The study site in Ldk. Gö. can be recommended for the establishment of KUP and AF considering natural characteristics and $\mathrm{N}$-balance. 


\section{Verzeichnisse}

\subsection{Abbildungen}

Abb. 1 Vereinfachter Stickstoffkreislauf (N-Kreislauf) mit Angaben zu Vorräten und Flüssen, die in dieser Arbeit bestimmt bzw. modelliert wurden, in grün: $\mathrm{N}$-Vorrat $\left(\mathrm{N}_{\text {org }}=\right.$ organischer Stickstoff [eON], $\mathrm{N}_{\min }=$ mineralischer Stickstoff [eIN], blau: N-Eintrag (Deposition und N-Nettomineralisation) und rot: N-Austrag (Auswaschung mit dem Sickerwasser, Denitrifikation: Lachgasemission und Ernte der holzigen Biomasse) $\mathbf{3}$

Abb. 2 Überblick zu den räumlichen Begriffen und Anbausystemen .......................................................... 7

Abb. 3 Räumliche Struktur des Landkreises Göttingen mit Lage der Versuchsfläche ...................................... 10

Abb. 4 Räumliche Struktur der Thüringer Ackerebene mit Lage der Versuchsfläche....................................... 11

Abb. 5 Monatswerte von Temperatur (rot) und Niederschlag (blau) der DWD-Station Göttingen. Punkte zeigen langjährige Monatswerte (Periode 1991-2010) mit den absoluten Minima und Maxima als Schattierungen. Die Linien mit Kreuzen repräsentieren jeweils Temperatur und Niederschlag für 2011, das Etablierungsjahr der Kurzumtriebsplantagen und Agroforstsysteme....

Abb. 6 Monatswerte von Temperatur (rot) und Niederschlag (blau) der DWD-Station Dachwig. Punkte zeigen langjährige Monatswerte (Periode 1991-2010) mit den absoluten Minima und Maxima als Schattierungen. Die Linien mit Kreuzen repräsentieren jeweils Temperatur und Niederschlag für 2011, das Etablierungsjahr der Kurzumtriebsplantagen und Agroforstsysteme.....

Abb. 7 Flächendesign der Versuchsflächen Reiffenhausen (links) und BERTA (rechts) mit Angaben zum Jahr der Bestandsbegründung und der verwendeten Klone. Lage der Leitprofile. Datengrundlage: Reiffenhausen: Digitales Orthophoto 2010, Quelle: Auszug aus den Geobasisdaten der Niedersächsischen Vermessungsund Katasterverwaltung, (C) 2011 Landesamt für Geoinformation und Landentwicklung Niedersachsen (LGLN); BERTA: Digitales Orthophoto 2008, Quelle: @ GeoBaisDE / TLVermGeo

Abb. 8 Lage der Leitprofile und der Punkte für die Feinkartierung in Reiffenhausen (links) und BERTA (rechts). Datengrundlage: Reiffenhausen: Digitales Orthophoto 2010, Quelle: Auszug aus den Geobasisdaten der Niedersächsischen Vermessungs- und Katasterverwaltung, (C) 2011 Landesamt für Geoinformation und Landentwicklung Niedersachsen (LGLN), BERTA: Digitales Orthophoto 2008, Quelle: C GeoBaisDE / TLVermGeo.

Abb. 9 Lokalisation der Beprobungsbereiche auf den Versuchsflächen Reiffenhausen (links) und BERTA (rechts). Datengrundlage: Reiffenhausen: Digitales Orthophoto 2010, Quelle: Auszug aus den Geobasisdaten der Niedersächsischen Vermessungs- und Katasterverwaltung, (c) 2011 Landesamt für Geoinformation und Landentwicklung Niedersachsen (LGLN), BERTA: Digitales Orthophoto 2008, Quelle: (C) GeoBaisDE / TLVermGeo.

Abb. 10 Überblick zu den beprobten Doppelreihen in Reiffenhausen (links) und BERTA (rechts) von denen Blätter für die Bestimmung des Ernährungszustandes der Bestände gewonnen wurden. Datengrundlage: Reiffenhausen: Digitales Orthophoto 2010, Quelle: Auszug aus den Geobasisdaten der Niedersächsischen Vermessungs- und Katasterverwaltung, (c) 2011 Landesamt für Geoinformation und Landentwicklung Niedersachsen (LGLN)

Abb. 11 Verlauf der mittleren monatlich extrahierbaren mineralischen Stickstoffmenge (eIN) für die Versuchsfläche Reiffenhausen von Oktober 2011 bis Oktober 2012 ( $n=3$ pro Teilfläche und Monat, im Oberboden $0-30 \mathrm{~cm}$ )

Abb. 12 Verlauf der mittleren monatlich extrahierbaren mineralischen Stickstoffmenge (eIN) für die Versuchsfläche BERTA von Oktober 2011 bis Oktober 2012 ( $n=3$ pro Teilfläche und Monat, im Oberboden $0-30 \mathrm{~cm})$......

Abb. 13 Verlauf der mittleren monatlichen N-Nettomineralisationsmengen im Zeitraum März bis Oktober 2012 für die Versuchsfläche Reiffenhausen ( $n=4$ pro Monat und Teilfläche)

Abb. 14 Verlauf der mittleren monatlichen N-Nettomineralisationsmengen im Zeitraum März bis Oktober 2012 für die Versuchsfläche BERTA ( $n=4$ pro Monat und Teilfläche) 
Abb. 15 Mittlerer (Standardabweichung) Trockengewichtsverlust des Pappel- und Weidenlaubes in den ausgebrachten Litterbags über einen Zeitraum von 12 Monaten in Reiffenhausen ( $\mathrm{n}=4$ pro Entnahmetermin, insgesamt 4 Entnahmetermine im vierteljährlichen Turnus: nach 3, 6, 9 und 12 Monaten)

Abb. 16 Mittlerer (Standardabweichung) Trockengewichtsverlust des Pappel- und Weidenlaubes in den ausgebrachten Litterbags über einen Zeitraum von 12 Monaten in BERTA ( $n=4$ pro Entnahmetermin, insgesamt 4 Entnahmetermine im vierteljährlichen Turnus: nach 3, 6, 9 und 12 Monaten)

Abb. 17 Mittlere (positive Standardabweichung) jährliche Nitratkonzentration im Bodenwasser in $60 \mathrm{~cm}$ Bodentiefe in Reiffenhausen (Entnahmezeitpunkte an denen Bodenlösung gewonnen wurde: KUP-Pappel: $n=6$, Acker: $n=8$, AF-GL: $n=6$, AF-Weide: $n=4$, KUP-Weide: $n=4$ ) für den Zeitraum Dezember 2011 bis November 2012 51

Abb. 18 Mittlerer monatlicher Nitrataustrag für die Teilflächen KUP-Pappel (blau), KUP-Weide (orange) und AFWeide (rot) in $60 \mathrm{~cm}$ Bodentiefe für die Versuchsfläche Reiffenhausen, die durchgezogenen Linien und gefüllten Symbole sind tatsächlich gemessene Werte ( $\mathrm{DNO}_{3}$-Konzentrationen im Bodenwasser), gestrichelte Linien und ungefüllte Symbole bedeuten interpolierte Werte $\left(\mathrm{DNO}_{3}\right.$-Konzentrationen im Bodenwasser)

Abb. 19 Mittlere (positive Standardabweichung) jährliche Nitratkonzentration im Bodenwasser in $60 \mathrm{~cm}$ Bodentiefe in BERTA (Entnahmezeitpunkte an denen Bodenlösung gewonnen wurde: KUP-Pappel: $n=5$, Acker: $n=6$, AF-GL: $n=5$, AF-Weide: $n=4$, KUP-Weide: $n=6$ ) für den Zeitraum Januar 2012 bis Dezember 2012

Abb. 20 Mittlerer monatlicher Nitrataustrag für die Teilflächen KUP-Pappel (blau), KUP-Weide (orange) und AFWeide (rot) in $60 \mathrm{~cm}$ Bodentiefe für die Versuchsfläche BERTA, die durchgezogenen Linien und gefüllten Symbole sind tatsächlich gemessene Werte ( $\mathrm{DNO}_{3}$-Konzentrationen im Bodenwasser), gestrichelte Linien und ungefüllte Symbole bedeuten interpolierte Werte ( $\mathrm{DNO}_{3}$-Konzentrationen im Bodenwasser)..... 


\subsection{Tabellen}

Tabelle 1 Standortsdaten der Untersuchungsregionen: Landkreis Göttingen (Ldk. Gö.) und Thüringer Ackerebene.....

Tabelle 2 Übersicht zum Flächendesign der neu angelegten Versuchsflächen Reiffenhausen und BERTA ......... 16

Tabelle 3 Bewertungsklassen für die Vorräte an Gesamtstickstoff $\left(N_{t}\right)$, organischen Kohlenstoff $\left(C_{\text {org }}\right)$, Kalium $(\mathrm{K})$, Calcium (Ca), Magnesium $(\mathrm{Mg})$ und Gesamtphosphor $\left(\mathrm{P}_{\mathrm{t}}\right)$ und Bewertungsklassen der potenziellen Kationenaustauschkapazität $\left(\mathrm{KAK}_{\text {pot }}\right)$ im effektiven Wurzelraum .....

Tabelle 4 Mittlere (Standardabweichung) bodenchemische Kennwerte $(n=3)$ : $\mathrm{pH}$-Wert; Gesamtgehalt und Vorrat an $\mathrm{C}_{\text {org }}, \mathrm{N}_{\mathrm{t}}$, Gehalt an $\mathrm{CaMg}\left(\mathrm{CO}_{3}\right)_{2}$ und $\mathrm{CaCO}_{3}$ sowie $\mathrm{C}_{\text {org }} / \mathrm{N}_{\mathrm{t}}-$ Verhältnis der Leitprofile (LP) Reiffenhausen Referenz (RR), Reiffenhausen Weide (RW) und BERTA (B)

Tabelle 5 Mittlere (Standardabweichung) bodenchemische Kennwerte $(n=3)$ : Potenzielle Kationenaustauschkapazität $\left(\mathrm{KAK}_{\text {pot }}\right)$, Basensättigung (BS) und Kationensättigung sowie Vorrat an Natrium $(\mathrm{Na})$, Kalium (K), Calcium (Ca), Magnesium (Mg) und Gesamtphosphor $\left(P_{t}\right)$ der Leitprofile (LP) Reiffenhausen Referenz (RR), Reiffenhausen Weide (RW) und BERTA (B) ....

Tabelle 6 Bodenchemische Kennwerte der Feinkartierung: $\mathrm{pH}$-Wert; Gesamtgehalt und Vorrat an $\mathrm{C}_{\text {org }}, \mathrm{N}_{\mathrm{t}}, \mathrm{P}_{\mathrm{t}}$, Gehalt an $\mathrm{CaMg}\left(\mathrm{CO}_{3}\right)_{2}$ und $\mathrm{C}_{\text {org }} / \mathrm{N}_{\mathrm{t}}$-Verhältnis in Reiffenhausen

Tabelle 7 Bodenchemische Kennwerte der Feinkartierung: Potenzielle Kationenaustauschkapazität (KAK pot), Basensättigung (BS) und Kationensättigung sowie Vorrat an Natrium (Na), Kalium (K), Calcium (Ca) und Magnesium (Mg) in Reiffenhausen

Tabelle 8 Bodenchemische Kennwerte der Feinkartierung: $\mathrm{pH}$-Wert; Gesamtgehalt und Vorrat an $\mathrm{C}_{\text {org }}, \mathrm{N}_{\mathrm{t}}, \mathrm{P}_{\mathrm{t}}$, Gehalt an $\mathrm{CaCO}_{3}$ und $\mathrm{C}_{\text {org }} / \mathrm{N}_{\mathrm{t}}$-Verhältnis in BERTA

Tabelle 9 Bodenchemische Kennwerte der Feinkartierung: Potenzielle Kationenaustauschkapazität $\left(K A K_{\text {pot }}\right)$, Basensättigung (BS) und Kationensättigung sowie Vorrat an Natrium (Na), Kalium (K), Calcium (Ca) und Magnesium (Mg) in BERTA.

Tabelle 10 Mittlere (Standardabweichung) bodenchemische Kennwerte der Feinkartierung ( $n=9)$ : pH-Wert; Gehalt und Vorrat an $\mathrm{C}_{\text {org }}, \mathrm{N}_{\mathrm{t}}$, Gehalt an $\mathrm{CaMg}\left(\mathrm{CO}_{3}\right)_{2}$ und $\mathrm{CaCO}_{3}$ sowie $\mathrm{C}_{\text {org }} / \mathrm{N}_{\mathrm{t}}-$ Verhältnis der Flächen Reiffenhausen (R) und BERTA (B)

Tabelle 11 Mittlere (Standardabweichung) bodenchemische Kennwerte der Feinkartierung ( $n=9$ ): Potenzielle Kationenaustauschkapazität $\left(\mathrm{KAK}_{\text {pot }}\right)$, Basensättigung (BS) und Kationensättigung sowie Vorrat an Natrium $(\mathrm{Na})$, Kalium (K), Calcium (Ca), Magnesium (Mg) und Gesamtphosphor $\left(P_{t}\right)$ der Flächen Reiffenhausen (R) und BERTA (B)

Tabelle 12 Mittlere (Standardabweichung der monatlichen Mittelwerte) jährlich extrahierbare mineralische Stickstoffmenge (eIN, Oktober 2011 bis September 2012) im Oberboden (0-30 cm, n=36 pro Teilfläche) 40

Tabelle 13 Mittlere (Standardabweichung der monatlichen Mittelwerte) extrahierbare organische Stickstoffmenge (Oktober 2011 bis September 2012) im Oberboden (0-30 cm, n=36 pro Teilfläche).

Tabelle 14 Mittlere (Standardabweichung der monatlichen Mittelwerte) N-Nettomineralisationsmenge in kg (N) ha ${ }^{-1}$ für den Zeitraum März bis Oktober 2012 für die Versuchsflächen Reiffenhausen und BERTA ( $n=32$ pro Teilfläche).

Tabelle 15 Anzahl der vitalen und nachgesteckten Bäume pro ha und Anwuchsrate in Reiffenhausen und BERTA auf den verschiedenen Teilflächen (KUP-Pappel, KUP-Weide, AF-Weide, Pflanzung im Frühjahr 2011, Vollaufnahme Frühjahr 2012)

Tabelle 16 Mittlere Höhe (Standardabweichung), Brusthöhendurchmesser (BHD, Standardabweichung), Ertrag und Zuwachs der Pappeln und Weiden in Reiffenhausen und BERTA auf den Teilflächen (KUP-Pappel, KUPWeide, AF-Weide, $n=240$ pro Teilfläche Reiffenhausen, $n=$ mind. 160 pro Teilfläche BERTA) nach zweijähriger Standdauer

Tabelle 17 Mittlere Nährstoffgehalte in Blättern (Standardabweichung) der Pappeln und Weiden in KUP und AF in Reiffenhausen (Reiff.) und BERTA 2012 ( $n=$ mindestens 5 pro Teilfläche und Jahr, Optimum nach Röhricht u. Ruscher 2009, Ausreichend nach Jug et al. 1999a). 
Tabelle 18 Jährlicher Zuwachs an holziger Biomasse, mittlere (Standardabweichung, SD) Nährstoffvorräte im Boden (austauschbare Kationen und $P_{t}, 0-30 \mathrm{~cm}$ Bodentiefe), mittlere (SD) eIN-Menge $(0-30 \mathrm{~cm})$ sowie mittlere (SD) jährliche Nährstofffestlegung in der holzigen Biomasse für die verschiedenen Teilflächen (KUP-Pappel, KUP-Weide, AF-Weide) in Reiffenhausen (Reiff.) und BERTA

Tabelle 19 Mittlere (Standardabweichung) Nährstoffgehalte und C/N- bzw. C/P-Verhältnis des eingetragenen Laubes im Herbst 2011 und 2012 für die Versuchsflächen Reiffenhausen und BERTA ( $\mathrm{n}=6$ pro Jahr und Teilfläche)

Tabelle 20 Mittlere (Standardabweichung) jährlich eingetragene Nährstoffmenge mit dem Laub ( $n=6$ pro Jahr und Teilfläche) in Reiffenhausen und BERTA

Tabelle 21 Mittlere (Standardabweichung) Nährstoffgehalte des ausgebrachten Laubes in Litterbags zu Beginn und am Ende des Versuchs nach einjähriger Ausbringungszeit ( $n=4$ pro Teilfläche und Zeitpunkt: Beginn bzw. Ende) in Reiffenhausen (Reiff.) und BERTA

Tabelle 22 Mittlere tägliche $\left(\mathrm{k} \mathrm{d}^{-1}\right)$ und jährliche Zersetzungsrate $\left(\mathrm{k}^{-1}\right)$ des Weiden- und Pappellaubes in KUP und AF ( $n=4$ pro Teilfläche) in Reiffenhausen und BERTA.

Tabelle 23 Mittlerer (Standardabweichung) Trockengewichtsverlust und mittlere (Standardabweichung) Nährstofffreisetzung in \% nach 12 Monaten für Pappel- und Weidenlaub in KUP und AF ( $\mathrm{n}=4$ pro Teilfläche) in Reiffenhausen und BERTA

Tabelle 24 Mittlerer (Standardabweichung) jährlicher Ein- und Austrag (Flüsse) an Stickstoff (N) und Stickstoffbilanz (N-Bilanz) für die verschiedenen holzigen Anbausysteme (KUP-Pappel, KUP-Weide, AFWeide) in Reiffenhausen (Reiff.) und BERTA

Tabelle 25 Stickstoffnutzungseffizienz: Stickstoffaufnahmeeffizienz und Stickstoffzuwachseffizienz [kg kg ${ }^{-1}$ ], berechnet aus dem Zuwachs an holziger Biomasse und den mittleren jährlichen Stickstoffflüssen für die verschiedenen holzigen Anbausysteme in Reiffenhausen (Reiff.) und BERTA. 


\subsection{Literatur}

Adegbidi H. G., Volk T. A., White E. H., Abrahamson L., Briggs R. D., Bickelhaupt D. H. 2001. Biomass and nutrient removal by willow clones in experimental bioenergy plantations in New York State. Biomass and Bioenergy 20 (6), 399-411

Ad-Hoc-Arbeitsgruppe Boden 2005. Bodenkundliche Kartieranleitung. Hannover

AdV - Arbeitsgemeinschaft der Vermessungsverwaltungen der Länder der Bundesrepublik Deutschland 2003. Amtliches Topographisch-Kartographisches Informationssystem - ATKIS - Objektartenkatalog Basis DLM. München

Albers D., Migge S., Schaefer M., Scheu S. 2004. Decomposition of beech leaves (Fagus sylvatica) and spruce needles (Picea abies) in pure and mixed stands of beech and spruce. Soil Biology and Biochemistry 36 (1), 155-164

Arbeitskreis Standortskartierung 2003. Forstliche Standortsaufnahme. München

Aretz A., Hirschl B. 2008. Eine Option zur Angebotserhöhung und ihre ökologischen Wirkungen. Holz von landwirtschaftlichen Flächen. Ökologisches Wirtschaften 23 (1), 19-21

Arp G., Tanner D., Leiss B. 2011a. Struktur der Leinetalgraben-Randstörung bei Reiffenhausen (Autobahn 38 Heidkopftunnel-Westportal). In: Leiss B., Tanner D., Vollbrecht A., Arp G. (Hrsg.): Neue Untersuchungen zur Geologie der Leinetalgrabenstruktur. Bausteine zur Erkundung des geothermischen Potenzials der Region Göttingen. Göttingen, 17-21

Arp G., Vollbrecht A., Tanner D., Leiss B. 2011b. Zur Geologie des Leinetalgrabens - ein kurzer Überblick. In: Leiss B., Tanner D., Vollbrecht A., Arp G. (Hrsg.): Neue Untersuchungen zur Geologie der Leinetalgrabenstruktur. Bausteine zur Erkundung des geothermischen Potenzials der Region Göttingen. Göttingen, 1-7

Asplund L., Bergkvist G., Weih M. 2014. Proof of concept: nitrogen use efficiency of contrasting spring wheat varieties grown in greenhouse and field. Plant and Soil 374 (1-2), 829-842

Aust C. 2012. Abschätzung der nationalen und regionalen Biomassepotentiale von Kurzumtriebsplantagen auf landwirtschaftlichen Flächen in Deutschland. http://www.freidok.uni-freiburg.de/volltexte/8630/. Aufgerufen am 27.6.2013

Balasus A., Bischoff W.-A., Schwarz A., Scholz V., Kern J. 2012. Nitrogen fluxes during the initial stage of willows and poplars in short-rotation coppices. Journal of Plant Nutrition and Soil Science 175 (5), 729-738

Bärlocher F. 2005. Leaf Mass Loss Estimated by Litter Bag Technique. In: Graças M. A. S., Bärlocher F., Gessner M. O. (Hrsg.): Methods to study litter decomposition. A practical guide. Dordrecht, New York, 3742

Bärwolff M., Hansen H., Hofmann M., Setzer F. 2012. Energieholz aus der Landwirtschaft. http://mediathek.fnr.de/energieholz-aus-der-landwirtschaft.html. Aufgerufen am 3.12.2013

Baum C., Eckhardt K.-U., Hahn J., Weih M., Dimitriou I., Leinweber P. 2013. Impact of poplar on soil organic matter quality and microbial communities in arable soils. Plant Soil Environ. 59 (3), 95-100

Baum C., Leinweber P., Weih M., Lamersdorf N., Dimitriou I. 2009. Effects of short rotation coppice with willow and poplar on soil ecology. vTI Landbauforschung 59 (3), 183-196

Bemmann A., Feger K.-H., Gerold D., Hartmann K.-U., Petzold R., Röhle H., Schweinle J., Steinke C. 2007. Kurzumtriebsplantagen auf landwirtschaftlichen Flächen in der Region Großenhain im Freistaat Sachsen. Forstarchiv 78 (3), 95-101

Bemmann A., Nahm M., Brodbeck F., Sauter U. 2010. Holz aus Kurzumtriebsplantagen: Hemmnisse und Chancen. Forstarchiv 81 (6), 246-254

Bergante S., Facciotto G., Minotta G. 2010. Identification of the main site factors and management intensity affecting the establishment of Short-Rotation-Coppices (SRC) in Northern Italy through stepwise regression analysis. Central European Journal of Biology 5 (4), 522-530 
Berthelot A., Ranger J., Gelhaye D. 2000. Nutrient uptake and immobilization in a short-rotation coppice stand of hybrid poplars in north-west France. Forest Ecology and Management 128 (3), 167-179

Bielefeldt J., Bolte A., Busch G., Dohrenbusch A., Kroiher F., Lamersdorf N., Schulz U., Stoll B. 2008. Energieholzproduktion in der Landwirtschaft. Chancen und Risiken aus Sicht des Natur- und Umweltschutzes. http://www.nabu.de/imperia/md/content/nabude/energie/biomasse/nabustudie_energieholz.pdf. Aufgerufen am 6.12.2013

Biertümpfel A., Rudel H., Werner A., Vetter A. 2009.15 Jahre Energieholzversuche in Thüringen. http://www.tll.de/ainfo/pdf/ehol1009.pdf. Aufgerufen am 3.12.2013

Blume H.-P., Brümmer G. W., Horn R., Kandeler E., Kögel-Knabner I., Kretzschmar R., Stahr K., Thiele-Bruhn S., Welp G., Wilke B.-M., Scheffer F., Schachtschabel P. 2010. Lehrbuch der Bodenkunde. Heidelberg

Blume H.-P., Stahr K., Leinweber P. 2011. Bodenkundliches Praktikum: Eine Einführung in pedologisches Arbeiten für Ökologen, insbesondere Land- und Forstwirte, und für Geowissenschaftler. Heidelberg

Boelcke B. 2006. Schnellwachsende Baumarten auf landwirtschaftlichen Flächen. Leitfaden zur Erzeugung von Energieholz.

http://www.dendrom.de/daten/downloads/boelcke_leitfaden\%20energieholz.pdf. Aufgerufen am 10.5.2014

Boelcke B., Kahle P. 2008. Energieholzproduktion mit Weiden und Pappeln - Ertragsbildung und Grundnährstoff. Pflanzenbauwissenschaften 12 (2), 78-85

Bowersox T., Stover L., Strauss C., Blankenhorn P. 1992. Advantages of an effective weed control program for Populus hybrids. Tree Plant Notes 43 (3), 81-86

Breitschuh T., Gernand U. Nachhaltigkeit im Maisanbau sichern. http://bioenergie.fnr.de/fileadmin/bioenergieberatung/sachsen-anhalt/dateien/Nachhaltigkeit_im_Maisanbau.pdf. Aufgerufen am 10.3.2014

Bungart R., Hüttl R. F. 2004. Growth dynamics and biomass accumulation of 8-year-old hybrid poplar clones in a short-rotation plantation on a clayey-sandy mining substrate with respect to plant nutrition and water budget. Eur J Forest Res 123 (2), 105-115

Calfapietra C., Angelis P. d., Gielen B., Lukac M., Moscatelli M. C., Avino G., Lagomarsino A., Polle A., Ceulemans R., Mugnozza G. S., Hoosbeek M. R., Cotrufo M. F. 2007. Increased nitrogen-use efficiency of a short-rotation poplar plantation in elevated $\mathrm{CO}_{2}$ concentration. Tree Physiology 27 (8), 1153-1163

Chen X.-Y., Wu L.-H., Cao X.-C., Zhu Y.-H. 2013. Organic nitrogen components in soils from southeast China. Journal of Zhejiang University SCIENCE B 14 (4), 259-269

Chmara I., Knoblauch S., Ziegler G. 2001. Untersuchungsergebnisse zur atmosphärischen Deposition und ihrer Auswirkungen im Freistaat Thüringen. In: Ihle P. (Hrsg.): Atmosphärische Depositionen in der Bundesrepublik Deutschland, 140-160

Christou M., Avramides E., Roberts J., Jones D. 2005. Dissolved organic nitrogen in contrasting agricultural ecosystems. Soil Biology and Biochemistry 37 (8), 1560-1563

Coll L., Messier C., Delagrange S., Berninger F. 2007. Growth, allocation and leaf gas exchanges of hybrid poplar plants in their establishment phase on previously forested sites: effect of different vegetation management techniques. Annals of Forest Science 64 (3), 275-285

Cornelissen J. H. C. 1996. An Experimental Comparison of Leaf Decomposition Rates in a Wide Range of Temperate Plant Species and Types. Journal of Ecology 84 (4), 573-582

Corre M. D., Dechert G., Veldkamp E. 2006. Soil Nitrogen Cycling following Montane Forest Conversion in Central Sulawesi, Indonesia. Soil Science Society of America Journal 70 (2), 359-366

Cotrufo M. F., Angelis P. de, Polle A. 2005. Leaf litter production and decomposition in a poplar short-rotation coppice exposed to free air $\mathrm{CO}_{2}$ enrichment (POPFACE). Global Change Biology 11 (6), 971-982

Dämmgen U., Grünhage L., Dörger G., Hanewald K. 2010. Beiträge zur Erstellung einer atmosphärischen Stickstoff-Bilanz für Hessen. http://www.hlug.de/fileadmin/dokumente/luft/faltblaetter/depo_faltblatt_mai2010.pdf. Aufgerufen am 11.6.2014 

http://www.deges.de/Ueber-DEGES/Weitere-Publikationen/DEGES-Publikationen-K113.htm. Aufgerufen am 13.11.2013

Devito K. J., Westbrook C. J., Schiff S. L. 1999. Nitrogen mineralization and nitrification in upland and peatland forest soils in two Canadian Shield catchments. Canadian Journal of Forest Research 29 (11), 17931804

DVWK - Deutscher Verband für Wasserwirtschaft und Kulturbau 1990. DVWK Merkblatt 217/1990. Gewinnung von Bodenwasserproben mit Hilfe der Saugkerzen-Methode.

Ehret M., Graß R., Wachendorf M. 2013. Ertragsentwicklung in der Etablierung eines Agroforstsystems aus Grünland und Weiden im Kurzumtrieb. In: FNR - Fachagentur Nachwachsende Rohstoffe e.V (Hrsg.): Gülzower Fachgespräche Agrarholz - Kongress 2013 19./20. Februar 2013, Berlin, 369-376

Eiler T., Matuschek D. 2012. Wie hoch ist der Bodenvorrat 2012? http://landundforst.agrarheute.com/nminwintergetreide. Aufgerufen am 30.10.2013

Elowson S., Rytter L. 1988. Dynamics of leaf minerals, leaf area, and biomass from hardwoods intensively grown on a peat bog. Trees 2 (2), 84-91

Eno C. F. 1960. Nitrate Production in the Field by Incubating the Soil in Polyethylene Bags. Soil Science Society of America Journal 24 (4), 277-279

Ernst W., Weigel H. 1992. Naturkundliche Wanderungen in Thüringen. Marburg

Falchero L., Brown R. H., Mueller-Harvey I., Hanley S., Shield I., Karp A. 2011. Condensed tannins in willow (Salix spp.): a first step to evaluate novel feeds for nutritionally improved animal products. In: Pötsch $\mathrm{E}$. M., Krautzer B., Hopkins A. (Hrsg.): Grassland farming and land management systems in mountainous regions. Proceedings of the 16th Symposium of the European Grassland Federation, Gumpenstein, Austria, 29th-31st August, 2011

Fier A., Höper H., Lau K., Meesenburg H., Schäfer W. 2013. Quellen und Einträge. http://www.umwelt.niedersachsen.de/umweltbericht/herausforderungen/eutrophierung/naehrst offeintrag_durch_deposition/quellen_und_eintraege/quellen-und-eintraege-88992.html. Aufgerufen am 6.1.2013

Fierer N., Schimel J. P., Cates R. G., Zou J. 2001. Influence of balsam poplar tannin fractions on carbon and nitrogen dynamics in Alaskan taiga floodplain soils. Soil Biology and Biochemistry 33 (12-13), 18271839

Friedel J. K., Gabel D. 2001. Microbial biomass and microbial C:N ratio in bulk soil and buried bags for evaluating in situ net $\mathrm{N}$ mineralization in agricultural soils. Journal of Plant Nutrition and Soil Science 164 (6), 673-679

Gasche R., Butterbach-Bahl K., Papen H. 2002. Development and application of a method for determination of net nitrification rates. Plant and Soil 240 (1), 57-65

Gillon D., Joffre R., Ibrahima A. 1999. Can Litter Decomposability be predicted by near infrared reflectance spectroscopy? Ecology 80 (1), 175-186

Gisi U. 1997. Bodenökologie. Stuttgart

Goodlass G., Green M., Hilton B., McDonough S. 2007. Nitrate leaching from short-rotation coppice. Soil Use and Management 23 (2), 178-184

Gruenewald H., Brandt B. K., Schneider B. U., Bens O., Kendzia G., Hüttl R. F. 2007. Agroforestry systems for the production of woody biomass for energy transformation purposes. Ecological Engineering 29 (4), 319-328

Guo L., Sims R. 1999. Litter decomposition and nutrient release via litter decomposition in New Zealand eucalypt short rotation forests. Agriculture, Ecosystems and Environment 75 (1-2), 133-140

Guo L., Sims R. 2001. Eucalypt litter decomposition and nutrient release under a short rotation forest regime and effluent irrigation treatments in New Zealand: I. External effects. Soil Biology and Biochemistry 33 (10), 1381-1388 
Guo L., Sims R. 2002. Eucalypt litter decomposition and nutrient release under a short rotation forest regime and effluent irrigation treatments in New Zealand: II. internal effects. Soil Biology and Biochemistry 34 (7), 913-922

Gutachterausschuss Forstliche Analytik 2005. Handbuch Forstliche Analytik. Eine Loseblatt-Sammlung der Analysemethoden im Forstbereich. http://www.bmelv.de/SharedDocs/Downloads/Landwirtschaft/WaldJagd/Bodenzustandserhebung/Handbuch/HandbuchForstanalytikKomplett.pdf;jsessionid=D7253A CE02DC916AD297982F16E9AF5A.2_cid376?_blob=publicationFile. Aufgerufen am 1.7.2013

Hansen E., Netzer D. A. 1985. Weed control using herbicides in short-rotation intensively cultured poplar plantations. http://www.ncrs.fs.fed.us/pubs/rp/rp_nc260.pdf. Aufgerufen am 9.7.2014

Hartmann L., Ehret M., Richter F., Leitch C., Lamersdorf N. 2013. Ertragsschätzung von schnellwachsenden Gehölzen in Kurzumtriebs- und Agroforstsystemen während der Etablierungsphase im Rahmen des Verbundprojektes BEST. In: Pekrun C., Wachendorf M., Francke-Weltmann L. (Hrsg.): Tagung der Gesellschaft für Pflanzenbauwissenschaften e.V. mit der Technischen Universität München, der Bayrischen Landesanstalt für Landwirtschaft und dem Technologie- und Förderzentrum Straubing 04. bis 06.September 2013. Göttingen, 273-274

Hartmann L., Lamersdorf N. 2014a. Site conditions, initial growth and results on nutrient and litter cycling of newly installed short rotation coppice (SRC) and agroforestry systems (AFS) within the BEST project. In: Bemmann et al. (Hrsg.): Bioenergy from Dendromass for the Sustainable Development of Rural Areas, eingereicht

Hartmann L., Lamersdorf N. 2014b. Willow short rotation plantation as an alley cropping system - aspects on yield development and nutrient cycling. In: Palma J., Chalmin A., Burgess P., Smith J. (Hrsg.): European Agroforestry Conference: integrating science \& policy to promote agroforestry practice (2nd). Book of Abstracts, 226-229

Hartmann L., Richter F., Busch G., Ehret M., Jansen M., Lamersdorf N. 2014. Etablierung von Kurzumtriebsplantagen im Rahmen des Verbundprojekts BEST in Süd-Niedersachsen und MittelThüringen - Standorteigenschaften und anfängliche Erträge. Forstarchiv 85 (4), 126-142

Hein M. 2003. Stickstoff-Umsätze in Humusauflagen von Buchen- und Fichtenwäldern im Fichtelgebirge (NOBayern) und entlang eines europäischen Nord-Süd-Transekts. Dissertation. Jena

Herold L., Höpfner E. 2008. Zur Entwicklung des Nmin-Gehaltes in Thüringer Böden. In: Thüringer Landesanstalt für Landwirtschaft (Hrsg.): Untersuchungsbericht 2006/2007.

Heunisch C., Caspers G., Elbracht J., Langer A., Röhling H.-G., Schwarz C., Streif H. 2007. Erdgeschichte von Niedersachsen. Geologie und Landschaftsentwicklung. http://www.lbeg.niedersachsen.de/portal/live.php?navigation_id=582\&article_id=822\&_psmand= 4. Aufgerufen am 15.11.2013

Hofmann M., Amthauer Gallardo D., Siebert C. 2012. Verbundvorhaben ProLoc: Klon-StandortWechselwirkungen bei Pappel und Weide auf landwirtschaftlichen Standorten in kurzen Umtriebszeiten. In: Nordwestdeutsche Forstliche Versuchsanstalt (Hrsg.): Züchtung und Ertragsleistung schnellwachsender Baumarten im Kurzumtrieb. Erkenntnisse aus drei Jahren FastWOOD, ProLoc und Weidenzüchtung., 9-20

Hofmann-Schielle C., Makeschin F., Rehfuess K. 1999. Short-rotation plantations of balsam poplars, aspen and willows on former arable land in the Federal Republic of Germany. I. Site-growth relationships. Forest Ecology and Management 121 (1-2), 41-55

Hrynkiewicz K., Toljander Y. K., Baum C., Fransson P. M. A., Taylor A. F. S., Weih M. 2012. Correspondence of ectomycorrhizal diversity and colonisation of willows (Salix spp.) grown in short rotation coppice on arable sites and adjacent natural stands. Mycorrhiza 22 (8), 603-613

Ihl C. 2010. Schnellwachsende Baumarten auf landwirtschaftlichen Flächen in Niedersachsen. http://www.3n.info/index.php?con_kat=162\&con_art=1051\&con_lang=1\&idlay=3. Aufgerufen am 6.12.2013

Jacob M., Viedenz K., Polle A., Thomas F. M. 2010. Leaf litter decomposition in temperate deciduous forest stands with a decreasing fraction of beech (Fagus sylvatica). Oecologia 164 (4), 1083-1094 
Jones D., Healey J. R., Willett V. B., Farrar J. F., Hodge A. 2005. Dissolved organic nitrogen uptake by plants-an important $\mathrm{N}$ uptake pathway? Soil Biology and Biochemistry 37 (3), 413-423

Jørgensen U., Schelde K. 2001. Energy crop water and nutrient use efficiency. http://infohouse.p2ric.org/ref/17/16275.pdf. Aufgerufen am 28.2.2014

Jug A., Hofmann-Schielle C., Makeschin F., Rehfuess K. 1999a. Short-rotation plantations of balsam poplars, aspen and willows on former arable land in the Federal Republic of Germany. II. Nutritional status and bioelement export by harvested shoot axes. Forest Ecology and Management 121 (1-2), 67-83

Jug A., Makeschin F., Rehfuess K., Hofmann-Schielle C. 1999b. Short-rotation plantations of balsam poplars, aspen and willows on former arable land in the Federal Republic of Germany. III. Soil ecological effects. Forest Ecology and Management 121 (1-2), 85-99

Kaushal R., Verman K. 2003. Leaf litter decomposition in different agroforestry tree species as influenced by climatic variables and subtrate quality. http://www.fao.org/docrep/ARTICLE/WFC/XII/0464B5.HTM. Aufgerufen am 24.12.2013

Kleber M., Nikolaus P., Kuzyakov Y., Stahr K. 2000. Formation of mineral $\mathrm{N}\left(\mathrm{NH}_{4}{ }^{+}, \mathrm{NO}_{3}{ }^{-}\right)$during mineralization of organic matter from coal refuse material and municipal sludge. Journal of Plant Nutrition and Soil Science 163 (1), 73-80

Klinck U., Fröhlich D., Meiwes K., Beese F. 2013. Entwicklung der Stoffein- und -austräge nach einem FichtenKleinkahlschlag. Forstarchiv 84 (3), 93-101

Kölling C., Göttlein A., Rothe A. 2007. Energieholz nachhaltig nutzen. Biomassenutzung und Nährstoffentzug. LWF aktuell 61 14, 32-37

Korhonen J. F. J., Pihlatie M., Pumpanen J., Aaltonen H., Hari P., Levula J., Kieloaho A.-J., Nikinmaa E., Vesala T., Ilvesniemi H. 2013. Nitrogen balance of a boreal Scots pine forest. Biogeosciences 10 (2), 10831095

Kreutzer K., Butterbach-Bahl K., Rennenberg H., Papen H. 2009. The complete nitrogen cycle of an N-saturated spruce forest ecosystem. Plant Biology 11 (5), 643-649

Kuers K., Simmons J. 2003. Leaf Litter Decomposition. http://www.sewanee.edu/Forestry_Geology/watershed_web/Emanuel/DecompStudy/Decomp_SE T.html. Aufgerufen am 9.7.2014

Kuzyakov Y., Xu X. 2013. Competition between roots and microorganisms for nitrogen: mechanisms and ecological relevance. New Phytologist 198 (3), 656-669

Lamersdorf N., Busch G. 2010. Kurzumtriebsplantagen. Handlungsempfehlungen zur naturverträglichen Produktion von Energieholz in der Landwirtschaft. Ergebnisse aus dem Projekt NOVALIS. http://www.dbu.de/projekt_23892/_db_1036.html. Aufgerufen am 3.2.2014

Lamersdorf N., Petzold R., Schwärzel K., Feger K., Köstner B., Moderow U., Bernhofer C., Knust C. 2010. Bodenökologische Aspekte von Kurzumtriebsplantagen. In: Bemmann A., Knust C. (Hrsg.): AGROWOOD: Kurzumtriebsplantagen in Deutschland und europäische Perspektiven. Berlin, 170188

Lammel G., Flessa H. 1998. Anthropogene Störung des Stickstoff-Kreislaufs. Umweltwissenschaften und Schadstoff-Forschung 10 (5), 295-305

Landgraf D., Böcker L., Oldenburg C. 2007. Landwirte als Energieholz-Produzenten?! Praxisrelevante Ernte einer Kurzumtriebsplantage. AFZ-DerWald (14), 751-753

Landgraf D., Böcker L., Schildbach M., Wolf H. 2010. Baumarten- und Sortenwahl. In: Skodawessely C., Pretzsch J., Bemmann A. (Hrsg.): Beratungshandbuch zu Kurzumtriebsplantagen. Entscheidungsgrundlagen zur Etablierung von Kurzumtriebsplantagen in Deutschland. Dresden

LBEG - Landesamt für Bergbau Energie und Geologie 2012. NIBIS ${ }^{\circledR}$ - Kartenserver. http://nibis.lbeg.de/cardomap3/?TH=BGL500\#. Aufgerufen am 13.9.2012

Lee K.-H., Jose S. 2006. Nitrogen mineralization in short-rotation tree plantations along a soil nitrogen gradient. Canadian Journal of Forest Research 36 (5), 1236-1242 
Liebhard P. 2007. Energieholz im Kurzumtrieb: Rohstoff der Zukunft. Graz, Stuttgart

Lipson D., Näsholm T. 2001. The unexpected versatility of plants: organic nitrogen use and availability in terrestrial ecosystems. Oecologia 128 (3), 305-316

Lodhiyal L., Lodhiyal N. 1997a. Nutrient Cycling and Nutrient Use Efficiency in Short Rotation, High Density Central Himalayan Tarai Poplar Plantations. Annals of Botany 79 (5), 517-527

Lodhiyal L., Lodhiyal N. 1997b. Variation in biomass and net primary productivity in short rotation high density central Himalayan poplar plantations. Forest Ecology and Management 98 (2), 167-179

Lodhiyal L., Singh R., Singh S. 1995. Structure and Function of an Age Series of Poplar Plantations in Central Himalaya. II Nutrient Dynamics. Annals of Botany 76 (2), 201-210

Lord E. I., Shepherd M. 1993. Developments in the use of porous ceramic cups for measuring nitrate leaching. Journal of Soil Science 44 (3), 435-449

Lorenz K., Müller J. 2013. Ergebnisse zur Nettoprimärproduktion von mit biologisch geklärtem Abwasser bewässerten Pappeln und Weiden im Kurzumtrieb. Landbauforschung - Applied Agricultural and Forestry Research 63 (4), 307-320

LSKN - Landesbetrieb für Statistik und Kommunikationstechnologie Niedersachsen 2011. Bevölkerung der $\begin{array}{llll}\text { Gemeinden } & \text { am } & 30 . & \text { Juni }\end{array}$ http://www.Iskn.niedersachsen.de/portal/live.php?navigation_id=25688\&article_id=87679\&_psm and $=40$. Aufgerufen am 15.11.2013

Makeschin F., Rehfuess K., Rüsch I., Schörby R. 1989. Anbau von Pappeln und Weiden im Kurzumtrieb auf ehemaligen Acker: Standörtliche Voraussetzungen, Nährstoffversorgung, Wuchsleistung und bodenökologische Auswirkungen. Forstw. Cbl. 108 (1), 125-143

Matsumoto S., Ae N. 2004. Characteristics of extractable soil organic nitrogen determine using various chemical solutions and its significance for nitrogen uptake by crops. Soil Science and Plant Nutrition 50 (1), 1-9

Meiresonne L., Schrijver A. D., Vos B. de 2006. Nutrient cycling in a poplar plantation (Populus trichocarpa $\mathrm{x}$ Populus deltoïdes 'Beaupré') on former agricultural land in northern Belgium. NRC

Moll R. H., Kamprath E. J., Jackson W. A. 1982. Analysis and Interpretation of Factors Which Contribute to Efficiency of Nitrogen Utilization. Agronomy Journal 74 (3), 562-564

Murach D., Aretz A., Hirschl B. 2009. Agrarholz als neue Perspektive für Landwirte. Potenziale und Rahmenbedingungen schnellwachsender Baumarten. Ökologisches Wirtschaften 24 (1), $20-22$

Murphy D. V., Macdonald A. J., Stockdale E. A., Goulding K. W. T., Fortune S., Gaunt J. L., Poulton P. R., Wakefield J. A., Webster C. P., Wilmer W. S. 2000. Soluble organic nitrogen in agricultural soils. Biology and Fertility of Soils 30 (5-6), 374-387

Näsholm T., Kielland K., Ganeteg U. 2009. Uptake of organic nitrogen by plants. New Phytologist 182 (1), 31-48

Nationale Akademie der Wissenschaften 2012. Bioenergie: Möglichkeiten und Grenzen. Kurzfassung und Empfehlungen.

http://www.leopoldina.org/uploads/tx_leopublication/201207_Bioenergie_Stellungnahme_kurz_d e_en_Okt2012_02.pdf. Aufgerufen am 13.11.2013

Nationale Akademie der Wissenschaften 2013. Stellungnahme. Bioenergie - Möglichkeiten und Grenzen. http://www.leopoldina.org/uploads/tx_leopublication/2013_06_Stellungnahme_Bioenergie_DE.p df. Aufgerufen am 13.11.2013

Neff J. C., Chapin F. S., Vitousek P. M. 2003. Breaks in the cycle: dissolved organic nitrogen in terrestrial ecosystems. Frontiers in Ecology and the Environment 1 (4), 205-211

Neumann C. 2008. Bewertung von Stickstoffdeposition in Waldökosystemen im Umfeld von 15 sächsischen Tierhaltungsanlagen - Prüfung des Verfahrens nach Bund/Länderarbeitsgemeinschaft für Immissionsschutz (LAI). http://www.forsten.sachsen.de/wald/download/Abschlussbericht_LAIVerfahren70.pdf. Aufgerufen am 11.6.2014 
Neumann J. H. 1883. Erläuterungen zur geologischen Specialkarte von Preussen und den Thüringischen Staaten. Gradabteilung 56, No. 57. Blatt Gräfen-Tonna. Berlin

Olson J. S. 1963. Energy Storage and the Balance of Producers and Decomposers in Ecological Systems. Ecology $44(2), 322-331$

Pandey R., Sharma G., Tripathi S., Singh A. 2007. Litterfall, litter decomposition and nutrient dynamics in a subtropical natural oak forest and managed plantation in northeastern India. Forest Ecology and Management 240 (1-3), 96-104

Peh K. S.-H., Sonké B., Taedoung H., Séné O., Lloyd J., Lewis S. L. 2012. Investigating diversity dependence of tropical forest litter decomposition: experiments and observations from Central Africa. Journal of Vegetation Science 23 (2), 223-235

Pérez C. A., Hedin L. O., Armesto J. J. 1998. Original Articles: Nitrogen Mineralization in Two Unpolluted OldGrowth Forests of Contrasting Biodiversity and Dynamics. Ecosystems 1 (4), 361-373

Pérez-Corona M. E., Hernández M. C. P., Castro F. B. de 2006. Decomposition of Alder, Ash, and Poplar Litter in a Mediterranean Riverine Area. Communications in Soil Science and Plant Analysis 37 (7-8), 11111125

Petzold R. 2010. Allgemeine Standortseignung. In: Skodawessely C., Pretzsch J., Bemmann A. (Hrsg.): Beratungshandbuch zu Kurzumtriebsplantagen. Entscheidungsgrundlagen zur Etablierung von Kurzumtriebsplantagen in Deutschland. Dresden

Petzold R., Feger K.-H., Röhle H. 2010a. Standörtliche Voraussetzungen für Kurzumtriebsplantagen. In: Bemmann A., Knust C. (Hrsg.): AGROWOOD: Kurzumtriebsplantagen in Deutschland und europäische Perspektiven. Berlin, 44-53

Petzold R., Feger K.-H., Siemer B. 2006. Standörtliche Potenziale für den Anbau schnellwachsender Baumarten auf Ackerflächen. AFZ-DerWald 61 (16), 855-857

Petzold R., Schubert B., Feger K. 2010b. Biomasseproduktion, Nährstoffallokation und bodenökologische Veränderungen einer Pappel-Kurzumtriebsplantage in Sachsen (Deutschland). Die Bodenkultur 61 (3), 23-35

Polyakova O., Billor N. 2007. Impact of deciduous tree species on litterfall quality, decomposition rates and nutrient circulation in pine stands. Forest Ecology and Management 253 (1-3), 11-18

Powlson D. S. 1993. Understanding the soil nitrogen cycle. Soil Use and Management 9 (3), 86-93

Prescott C. E. 2005. Do rates of litter decomposition tell us anything we really need to know? Forest Ecology and Management $220(1-3), 66-74$

Prescott C. E. 2010. Litter decomposition: what controls it and how can we alter it to sequester more carbon in forest soils? Biogeochemistry 101 (1-3), 133-149

Rasmussen J., Sauheitl L., Eriksen J., Kuzyakov Y. 2010. Plant uptake of dual-labeled organic N biased by inorganic C uptake: Results of a triple labeling study. Soil Biology and Biochemistry 42 (3), 524-527

Rennenberg H., Dannenmann M., Gessler A., Kreuzwieser J., Simon J., Papen H. 2009. Nitrogen balance in forest soils: nutritional limitation of plants under climate change stresses. Plant Biology 11, 4-23

Rennenberg H., Wildhagen H., Ehlting B. 2010. Nitrogen nutrition of poplar trees. Plant Biology 12 (2), 275-291

Röhle H. 2009. Arbeitskreis Biomasse: Verfahrensempfehlungen zur Methodik der Biomasseermittlung in Kurzumtriebsbeständen. In: Nagel J. (Hrsg.): Jahrestagung 25.-27. Mai 2009, Ascona (Schweiz) Deutscher Verband Forstlicher Forschungsanstalten - Sektion Ertragskunde, 220-226

Röhle H. 2013. Standortleistungsschätzung und Biomasseermittlung in Kurzumtriebsplantagen. Allg. Forst- u. J.Ztg. 184 (11/12), 237-246

Röhle H., Böcker L., Feger K.-H., Petzold R., Wolf H., Ali W. 2008. Anlage und Ertragsaussichten von Kurzumtriebsplantagen in Ostdeutschland. Establishment and expected yield of short-term rotation plantations in Eastern Germany. Schweizerische Zeitschrift für Forstwesen 159 (6), 133139 
Röhle H., Hartmann K.-U., Gerold D., Steinke C., Schröder J. 2006. Aufstellung von Biomassefunktionen für Kurzumtriebsbestände. Allg. Forst- u. J.-Ztg. 177 (10/11), 178-187

Röhle H., Skibbe K. 2012. Ertragsschätzung in Kurzumtriebsplantagen aus Pappel und Weide. In: Nordwestdeutsche Forstliche Versuchsanstalt (Hrsg.): Züchtung und Ertragsleistung schnellwachsender Baumarten im Kurzumtrieb. Erkenntnisse aus drei Jahren FastWOOD, ProLoc und Weidenzüchtung., 105-116

Röhricht C., Grunert M., Ruscher K. 2011a. Feldstreifenanbau schnellwachsender Baumarten. https://publikationen.sachsen.de/bdb/artikel/15041. Aufgerufen am 3.12.2013

Röhricht C., Grunert M., Ruscher K. 2011b. Kurzumtriebsplantage Köllitsch. https://publikationen.sachsen.de/bdb/artikel/15076. Aufgerufen am 3.12.2013

Röhricht C., Ruscher K. 2009. Anbauempfehlungen. Schnellwachsende Baumarten im Kurzumtrieb. https://publikationen.sachsen.de/bdb/artikel/13410. Aufgerufen am 22.11.2013

Rösch C., Jorissen J. 2012. Perspektiven und Herausforderungen im Überblick - Hoffnungstrager Kurzumtriebsplantagen?Pinning Hopes on Short Rotation Coppice? Reviewing Perspectives and Challenges. GAIA - Ecological Perspectives for Science and Society 21 (3), 194-201

Ros G. H., Hoffland E., van Kessel C., Temminghoff E. J. 2009. Extractable and dissolved soil organic nitrogen - A quantitative assessment. Soil Biology and Biochemistry 41 (6), 1029-1039

Rytter L., Ericsson T. 1993. Leaf nutrient analysis in Salix viminalis (L.) energy forest stands growing on agricultural land. Z. Pflanzenernähr. Bodenk. 156 (4), 349-356

Schimel J., Bennett J. 2004. Nitrogen Mineralization: Challenges of a Changing Paradigm. Ecology 85 (3), $591-$ 602

Schimming C.-G., Mette R., Reiche E.-W., Schrautzer J., Wetzel H. 1995. Stickstoffflüsse in einem typischen Agrarökosystem Schleswig-Holsteins. Meßergebnisse, Bilanzen, Modellvalidierung. Z. Pflanzenernähr. Bodenk. 158 (4), 313-322

Schmidt-Walter P., Lamersdorf N. 2012. Biomass Production with Willow and Poplar Short Rotation Coppices on Sensitive Areas-the Impact on Nitrate Leaching and Groundwater Recharge in a Drinking Water Catchment near Hanover, Germany. Bioenerg Res 5 (3), 546-562

Scholz V., Boelcke B., Burger F., Hofmann M., Vetter A. 2006. KTBL-Datensammlung Energiepflanzen. MERKBLATT Produktion von Pappeln und Weiden auf landwirtschaftlichen Flächen. http://www.dendrom.de/daten/downloads/ktbl_merkblatt.pdf. Aufgerufen am 6.12.2013

Schwarz J., Bruderer Enzler H., Westphalen A. 2010. Methodenberatung. Kruskal-Wallis-Test. http://www.methodenberatung.uzh.ch/datenanalyse/unterschiede/zentral/kruskal.html. Aufgerufen am 23.1.2014

Sedlacek P. 2002. Thüringen. Geographische Exkursionen. Gotha

Seedorf H. H., Meyer H.-H. 1992. Landeskunde Niedersachsen. Natur- und Kulturgeschichte eines Bundeslandes. Band 1: Historische Grundlagen und naturräumliche Ausstattung. Neumünster

Seidel G. 2003. Geologie von Thüringen. Stuttgart

Seidel G., Unger K. 1995. Erläuterungen zur Geologischen Karte 1:25.000 von Thüringen. Blatt Erfurt NW, Nr. 4931. Weimar

Šlapokas T., Granhall U. 1991. Decomposition of willow-leaf litter in a short-rotation forest in relation to fungal colonization and palatability for earthworms. Biol Fertil Soils 10 (4), 241-248

Stauffer M., Leyval C., Brun J.-J., Leportier P., Berthelin J. 2014. Effect of willow short rotation coppice on soil properties after three years of growth as compared to forest, grassland and arable land uses. Plant and Soil 377 (1-2), 423-438

Stille H., Lotze F. 1933. Geologische Übersichtskarte der Umgebung von Göttingen 1:100.000 mit Erläuterungsheft. Göttingen, Berlin

Stoll B., Dohrenbusch A. 2009. Der Einfluss der Flächenvornutzung und Begleitwuchsregulierung auf den Anwuchserfolg von Energieholzplantagen. Allg. Forst- u. J.-Ztg. 181 (3/4), 71-76 
Strohm K., Schweinle J., Liesebach M., Osterburg B., Rödl A., Baum S., Nieberg H., Bolte A., Walter K. 2012. Kurzumtriebsplantagen aus ökologischer und ökonomischer Sicht, Arbeitsberichte aus der VTI-Agrarökonomie, No. 06/2012. http://nbn-resolving.de/urn:nbn:de:gbv:253-201210-dn0508572. Aufgerufen am 22.5.2014

Thevathasan N. V., Gordon A. M. 1997. Poplar leaf biomass distribution and nitrogen dynamics in a poplarbarley intercropped system in southern Ontario, Canada. Agroforestry Systems 37 (1), 79-90

Thüringer Landesanstalt für Landwirtschaft 2012. Aktueller Rat zur Nmin- und Smin-Situation Thüringer Böden im Frühjahr 2012. http://www.tll.de/ainfo/pdf/nmin0412.pdf. Aufgerufen am 3.2.2014

Thüringer Landesanstalt für Landwirtschaft 2013. Aktueller Rat zur Nmin- und Smin-Situation Thüringer Böden im Frühjahr 2013. http://www.tll.de/ainfo/pdf/nmin0513.pdf. Aufgerufen am 3.2.2014

TLS - Thüringer Landesamt für Statistik 2012. Bevölkerung der Gemeinden Thüringens am 30.06.2012. http://www.tls.thueringen.de/DatenBank/TabAnzeige.asp?tabelle=gg000201||. Aufgerufen am 15.8.2013

Toky O., Singh V. 1993. Litter dynamics in short-rotation high densitiy tree plantations in an arid region of india. Agriculture, Ecosystems and Environment 45 (1-2), 129-145

Tracy B. F., Frank D. A. 1998. Herbivore influence on soil microbial biomass and nitrogen mineralization in a northern grassland ecosystem: Yellowstone National Park. Oecologia 114 (4), 556-562

Tsonkova P., Böhm C., Quinkenstein A., Freese D. 2012. Ecological benefits provided by alley cropping systems for production of woody biomass in the temperate region: a review. Agroforestry Systems 85 (1), 133-152

Verwijst T., Telenius B. 1999. Biomass estimation procedures in short rotation forestry. Forest Ecology and Management 121 (1-2), 137-146

Volk T. A., Abrahamson L., Cameron K. D., Castellano P., Corbin T., Fabio E., Johnson G., Kuzovkina-Eischen Y., Labrecque M., Miller R., Sidders D., Smart L., Staver K., Stanosz G., van Rees K. 2011. Yields of willow biomass crops across a range of sites in North America. Aspects of Applied Biology 112, 6774

Vollbrecht A., Tanner D. 2011. Der Leinetalgraben als Teil einer regionalen Pull-Apart-Struktur. In: Leiss B., Tanner D., Vollbrecht A., Arp G. (Hrsg.): Neue Untersuchungen zur Geologie der Leinetalgrabenstruktur. Bausteine zur Erkundung des geothermischen Potenzials der Region Göttingen. Göttingen, 9-15

Wedell I. 2013a. Geotechnischer Längsschnitt. RE-Vorentwurf. A 38 Göttingen - Halle (A9), Verkehrseinheit Uder. Lgr. NI/TH - w AS Heiligenstadt. e-Mail Göttingen

Wedell I. 2013b. Lageplan. RE-Vorentwurf. A 38 Göttingen - Halle (A9), Verkehrseinheit Uder. Lgr. NI/TH - w AS Heiligenstadt. e-Mail Göttingen

Weih M., Asplund L., Bergkvist G. 2011. Assessment of nutrient use in annual and perennial crops: A functional concept for analyzing nitrogen use efficiency. Plant and Soil 339 (1-2), 513-520

Werner A., Vetter A., Reinhold G. 2006. Leitlinie zur effizienten und umweltverträglichen Erzeugung von Energieholz.

http://www.dendrom.de/daten/downloads/vetter_leitlinie\%20energieholz\%202006.pdf. Aufgerufen am 3.12.2013

Wunderlich H. 1964. Ausweitung und Einengung an saxonischen Bauformen Südniedersachsens. Zeitschrift der Deutschen Geologischen Gesellschaft. (116), 683-685

Zacios M., Niederberger J., Seidel H., Schulz C., Zimmermann L., Burger F. 2012. Hydrologische und ökologische Aspekte bei Kurzumtriebsplantagen. http://www.lwf.bayern.de/mitarbeiterverzeichnis/ae/burger/30737/linkurl_4.pdf. Aufgerufen am 28.1.2014

Ziegler P. A. 1990. Geological atlas of Western and Central Europe. The Hague 


\section{Anhang}

Anhang 1 Nährstoffkonzentrationen im Bodenwasser der verschiedenen Teilflächen in Reiffenhausen in $60 \mathrm{~cm}$ Bodentiefe, fehlende Werte sind durch zu wenig Lösung bedingt, angegeben sind Mittelwerte und in Klammern die zugehörige Standardabweichung (SD), falls keine SD angegeben, dann ist nur ein Analysewert vorhanden

\begin{tabular}{|c|c|c|c|c|c|c|c|c|c|}
\hline & & $\mathrm{pH}$ & $\mathrm{K}$ & $\mathrm{Ca}$ & $\mathrm{Mg}$ & $\mathrm{DNH}_{4}$ & $\mathrm{DNO}_{3}$ & DON & TDN \\
\hline & & & & & & {$\left[\mathrm{mg} \mathrm{L}^{-1}\right]$} & & & \\
\hline \multirow[t]{2}{*}{ Dez. 11} & AF-GL & 7,5 & & & & 0,0 & 0,4 & 1,1 & 1,5 \\
\hline & KUP-Weide & 8,1 & 0,5 & 82,8 & 27,9 & 0,0 & 30,3 & 2,2 & 32,5 \\
\hline \multirow[t]{2}{*}{ Jan. 12} & KUP-Pappel & 6,7 & 3,1 & 32,8 & 2,6 & 0,0 & 2,9 & 0,4 & 3,4 \\
\hline & AF-GL & $7,4(0,1)$ & $4,8(0,4)$ & $39,4(2,6)$ & $3,2(0,1)$ & $0,7(1,1)$ & $0,0(0,0)$ & $0,7(0,2)$ & $1,4(1,3)$ \\
\hline \multirow[t]{3}{*}{ März 12} & Acker & $8,0(0,0)$ & $10,2(1,7)$ & $59,4(7,5)$ & $4,3(0,7)$ & $0,0(0,0)$ & $1,2(1,1)$ & $0,3(0,1)$ & $1,5(1,1)$ \\
\hline & AF-GL & & 2,3 & 14,7 & 1,3 & & & & \\
\hline & KUP-Weide & $8,2(0,1)$ & $0,9(0,6)$ & $67,6(1,1)$ & $21,4(2,5)$ & $0,0(0,0)$ & $33,0(8,7)$ & $0,7(0,1)$ & $33,7(8,6)$ \\
\hline \multirow[t]{4}{*}{ April 12} & KUP-Pappel & & 4,2 & 40,4 & 3,0 & & & & \\
\hline & Acker & 7,7 & 12,4 & 63,8 & 5,0 & 0,0 & 1,3 & 0,3 & 1,6 \\
\hline & AF-Weide & 7,5 & 0,8 & 40,8 & 4,7 & 0,0 & 6,6 & 0,3 & 6,9 \\
\hline & KUP-Weide & $8,0(0,2)$ & $0,7(0,2)$ & $72,7(11,8)$ & $22,9(3,6)$ & $0,0(0,1)$ & $24,9(5,1)$ & $4,9(7,6)$ & $29,9(5,9)$ \\
\hline \multirow[t]{5}{*}{ Mai 12} & KUP-Pappel & $7,3(0,2)$ & $4,7(2,8)$ & $33,0(17,3)$ & $2,7(1,4)$ & $0,0(0,1)$ & $7,3(4,7)$ & $0,4(0,2)$ & $7,7(4,8)$ \\
\hline & Acker & $7,7(0,1)$ & $13,0(1,2)$ & $67,4(7,1)$ & $4,9(0,3)$ & $0,4(1,0)$ & $0,3(0,4)$ & $0,4(0,1)$ & $1,1(1,1)$ \\
\hline & AF-GL & $7,8(0,2)$ & $6,6(0,4)$ & $38,3(7,7)$ & $2,6(0,2)$ & $0,0(0,0)$ & $0,3(0,5)$ & $0,3(0,1)$ & $0,6(0,5)$ \\
\hline & AF-Weide & $7,6(0,1)$ & $1,3(0,7)$ & $26,6(15,8)$ & $3,7(2,0)$ & $0,3(0,5)$ & $2,1(2,1)$ & $0,3(0,3)$ & $2,7(2,5)$ \\
\hline & KUP-Weide & $8,3(0,0)$ & $1,0(0,4)$ & $64,8(7,6)$ & $22,5(3,8)$ & $0,0(0,0)$ & $21,2(3,9)$ & $2,4(3,2)$ & $24,1(6,5)$ \\
\hline \multirow[t]{3}{*}{ Juni 12} & KUP-Pappel & $7,5(0,3)$ & $6,3(2,4)$ & $42,3(12,8)$ & $3,3(0,9)$ & $0,0(0,0)$ & $5,4(4,8)$ & $0,6(0,2)$ & $6,0(4,9)$ \\
\hline & Acker & $7,6(0,1)$ & $12,1(0,9)$ & $64,9(4,7)$ & $4,7(0,3)$ & $1,2(1,8)$ & $0,1(0,1)$ & $0,5(0,1)$ & $1,8(2,0)$ \\
\hline & AF-GL & $7,8(0,1)$ & $5,5(1,1)$ & $39,8(8,8)$ & $2,6(0,2)$ & $0,0(0,0)$ & $0,0(0,0)$ & $0,3(0,1)$ & $0,3(0,1)$ \\
\hline \multirow[t]{3}{*}{ Juli 12} & KUP-Pappel & $7,9(0,3)$ & $6,8(0,6)$ & $54,9(10,2)$ & $3,8(0,2)$ & $0,0(0,0)$ & $0,5(0,8)$ & $0,5(0,1)$ & $1,0(1,0)$ \\
\hline & Acker & & 13,6 & 71,1 & 5,1 & 0,0 & 0,2 & 0,5 & 0,8 \\
\hline & AF-GL & 7,9 & 6,0 & 37,1 & 2,8 & 0,0 & 0,0 & 0,3 & 0,3 \\
\hline \multirow[t]{4}{*}{ Aug. 12} & KUP-Pappel & $8,0(0,1)$ & $6,7(2,6)$ & $44,7(9,5)$ & $3,4(0,7)$ & $0,0(0,0)$ & $0,0(0,0)$ & $0,4(0,1)$ & $0,4(0,1)$ \\
\hline & Acker & 8,0 & & & & 4,9 & 0,4 & 5,7 & 0,4 \\
\hline & AF-GL & 8,1 & 10,4 & 44,8 & 3,7 & 0,0 & 0,0 & 0,6 & 0,6 \\
\hline & AF-Weide & 8,0 & & & & 1,0 & 0,7 & 0,8 & 2,6 \\
\hline \multirow[t]{3}{*}{ Okt. 12} & KUP-Pappel & & & & & 0,5 & 0,0 & 0,7 & 1,2 \\
\hline & Acker & & 19,6 & 66,9 & 5,7 & 0,0 & 2,9 & 0,9 & 3,8 \\
\hline & AF-Weide & & & & & 0,0 & 2,2 & 0,7 & 2,9 \\
\hline Nov. 12 & Acker & 8,0 & & & & 0,0 & 5,8 & 0,4 & 6,2 \\
\hline
\end{tabular}


Anhang 2 Nährstoffkonzentrationen im Bodenwasser der verschiedenen Teilflächen in BERTA in $60 \mathrm{~cm}$ Bodentiefe, fehlende Werte sind durch zu wenig Lösung bedingt, angegeben sind Mittelwerte und in Klammern die zugehörige Standardabweichung (SD), falls keine SD angegeben, dann ist nur ein Analysewert vorhanden

\begin{tabular}{|c|c|c|c|c|c|c|c|c|c|}
\hline & & $\mathrm{pH}$ & $\mathrm{K}$ & $\mathrm{Ca}$ & $\mathrm{Mg}$ & $\mathrm{DNH}_{4}$ & $\mathrm{DNO}_{3}$ & DON & TDN \\
\hline & & & & & & {$\left[\mathrm{mg} \mathrm{L}^{-1}\right]$} & & & \\
\hline \multirow[t]{4}{*}{ März 12} & KUP-Pappel & 8,2 & 0,0 & 94,6 & 9,2 & 0,0 & 24,4 & 1,2 & 25,6 \\
\hline & Acker & 7,8 & 0,6 & 270,9 & 35,1 & 0,0 & 86,4 & 0,8 & 87,2 \\
\hline & AF-GL & $8,2(0,1)$ & $0,1(0,1)$ & $73,3(6,3)$ & $8,8(0,9)$ & $0,0(0,0)$ & $1,1(0,5)$ & $0,6(0,0)$ & $1,7(0,5)$ \\
\hline & KUP-Weide & 7,6 & 0,0 & 113,6 & 18,0 & 0,2 & 73,6 & 0,8 & 74,5 \\
\hline \multirow[t]{5}{*}{ April 12} & KUP-Pappel & $8,2(0,0)$ & $0,2(0,2)$ & $100,3(1,5)$ & $9,7(0,0)$ & $0,0(0,0)$ & $26,6(0,1)$ & $0,9(0,3)$ & $27,5(0,2)$ \\
\hline & Acker & $7,9(0,0)$ & $0,8(0,0)$ & $274,6(6,0)$ & $33,0(1,1)$ & $0,0(0,0)$ & $83,0(0,7)$ & $2,8(0,9)$ & $85,8(1,5)$ \\
\hline & AF-GL & $8,3(0,1)$ & $0,1(0,1)$ & $73,5(6,0)$ & $8,4(0,9)$ & $0,0(0,0)$ & $2,4(1,1)$ & $0,4(0,1)$ & $2,8(1,2)$ \\
\hline & AF-Weide & $8,3(0,1)$ & $0,1(0,2)$ & $68,1(3,0)$ & $8,8(0,9)$ & $0,0(0,1)$ & $8,2(1,0)$ & $0,4(0,2)$ & $8,6(1,0)$ \\
\hline & KUP-Weide & $8,0(0,2)$ & $0,1(0,2)$ & $126,1(14,0)$ & $16,7(1,3)$ & $0,0(0,0)$ & $65,7(14,7)$ & $2,2(1,4)$ & $68,0(14,4)$ \\
\hline \multirow[t]{5}{*}{ Mai 12} & KUP-Pappel & 8,2 & & & & $0,2(0,3)$ & $46,7(8,7)$ & $2,3(0,4)$ & $49,3(8,5)$ \\
\hline & Acker & $8,0(0,1)$ & $0,6(0,2)$ & $373,8(83,6)$ & $38,7(4,2)$ & $0,0(0,0)$ & $87,5(10,7)$ & $1,0(0,9)$ & $88,5(10,4)$ \\
\hline & AF-GL & $8,3(0,1)$ & $0,1(0,1)$ & $65,9(4,7)$ & $10,3(2,0)$ & $0,1(0,1)$ & $1,6(0,8)$ & $0,6(0,0)$ & $2,3(1,0)$ \\
\hline & AF-Weide & 8,4 & $0,0(0,1)$ & $60,1(6,7)$ & $8,9(1,0)$ & $0,0(0,0)$ & $8,5(1,4)$ & $1,2(0,8)$ & $9,7(0,7)$ \\
\hline & KUP-Weide & $8,0(0,1)$ & $0,2(0,2)$ & $128,2(14,5)$ & $17,8(1,2)$ & $0,0(0,0)$ & $72,2(13,6)$ & $3,4(2,1)$ & $75,6(14,2)$ \\
\hline \multirow[t]{5}{*}{ Juni 12} & KUP-Pappel & 8,2 & 2,1 & 110,0 & 14,1 & 0,0 & 50,9 & 0,7 & 51,6 \\
\hline & Acker & $8,0(0,1)$ & $1,0(0,4)$ & $319,7(82,3)$ & $36,3(3,6)$ & $0,0(0,0)$ & $81,4(1,2)$ & $2,0(1,4)$ & $83,4(1,3)$ \\
\hline & AF-GL & 8,3 & 0,4 & 54,9 & 9,5 & 0,0 & 4,4 & 0,6 & 5,1 \\
\hline & AF-Weide & & & & & 0,3 & 4,5 & 3,3 & 8,0 \\
\hline & KUP-Weide & $8,0(0,1)$ & $0,3(0,2)$ & $132,5(14,8)$ & $18,2(2,0)$ & $0,0(0,0)$ & $67,7(12,2)$ & $1,1(0,5)$ & $68,8(12,7)$ \\
\hline \multirow[t]{5}{*}{ Juli 12} & KUP-Pappel & $8,4(0,1)$ & $0,4(0,1)$ & $90,5(17,9)$ & $12,6(0,6)$ & $0,0(0,0)$ & $12,2(5,8)$ & $5,7(8,3)$ & $17,8(13,0)$ \\
\hline & Acker & $8,2(0,1)$ & $0,8(0,4)$ & $314,6(80,1)$ & $37,4(3,4)$ & $0,0(0,0)$ & $48,4(12,2)$ & $1,1(1,3)$ & $49,6(13,1)$ \\
\hline & AF-GL & 8,4 & 0,6 & 63,1 & 12,1 & 0,3 & 3,3 & 1,1 & 4,7 \\
\hline & AF-Weide & $8,4(0,0)$ & $0,2(0,1)$ & $86,0(8,4)$ & $12,7(1,7)$ & $0,0(0,0)$ & $0,0(0,0)$ & $0,6(0,2)$ & $0,6(0,2)$ \\
\hline & KUP-Weide & $8,4(0,1)$ & $0,2(0,0)$ & $105,1(17,9)$ & $16,6(2,9)$ & $0,0(0,0)$ & $26,4(9,6)$ & $1,1(0,6)$ & $27,5(9,8)$ \\
\hline \multirow[t]{2}{*}{ Aug. 12} & Acker & 8,2 & 0,6 & 378,6 & 40,6 & 0,0 & 42,6 & 2,1 & 44,7 \\
\hline & AF-Weide & $8,7(0,0)$ & 0,3 & 65,3 & 11,9 & $0,0(0,0)$ & $0,6(0,8)$ & $0,8(0,1)$ & $1,4(0,7)$ \\
\hline
\end{tabular}

